\title{
Development of Transgenic Sterile Insect Technique Strains for the Invasive Fruit Pest Drosophila suzukii
}

\author{
Dissertation \\ for the award of the degree \\ "Doctor rerum naturalium" (Dr. rer. nat.) \\ of the Georg-August-Universität Göttingen \\ within the doctoral program 'Biology' \\ of the Georg-August University School of Science (GAUSS) \\ submitted by \\ Hassan Mutasim Mohammed Ahmed \\ from Omdurman, Sudan
}

Göttingen, 2019

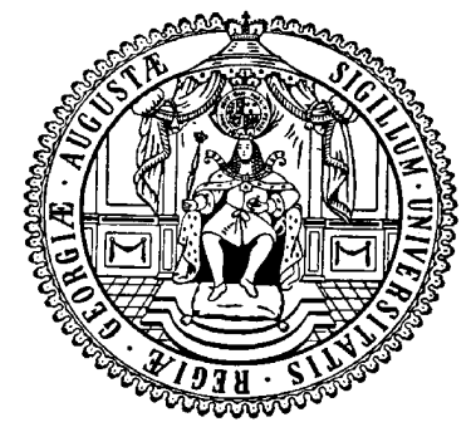





\section{Thesis Advisory Committee:}

Prof. Dr. Ernst A. Wimmer (Supervisor)

Entwicklungsbiologie - Johann-Friedrich-Blumenbach-Institut für Zoologie und Anthropologie Georg-August-Universität Göttingen

\section{Prof. Dr. Gregor Bucher}

Evolutionäre Entwicklungsgenetik - Johann-Friedrich-Blumenbach-Institut für Zoologie und Anthropologie -Georg-August-Universität Göttingen

\section{Members of the Examination Board:}

First Reviewer: Prof. Dr. Ernst A. Wimmer

Entwicklungsbiologie - Johann-Friedrich-Blumenbach-Institut für Zoologie und Anthropologie Georg-August-Universität Göttingen

Second Reviewer: Prof. Dr. Gregor Bucher

Evolutionäre Entwicklungsgenetik - Johann-Friedrich-Blumenbach-Institut für Zoologie und Anthropologie -Georg-August-Universität Göttingen

\section{Further members of the Examination Board:}

\section{Prof. Dr. Reinhad Schuh}

Molekulare Organogenese - Max Planck Institute for Biophysical Chemistry

\section{Prof. Dr. Ralf Heinrich}

Zelluläre Neurobiologie, Schwann-Schleiden Research Centre, Johann-Friedrich-BlumenbachInstitut für Zoologie und Anthropologie -Georg-August-Universität Göttingen

\section{Prof. Dr. Daniel Jackson}

Evolution der Metazoen, Geoscience Centre - Georg-August-Universität Göttingen

Dr. Roland Dosch

Entwicklungsbiochemie, Universitätsmedizin Göttingen 


\section{DECLARATION}

I hereby declare that the doctoral thesis entitled "Development of Transgenic Sterile Insect Technique Strains for the Invasive Fruit Pest Drosophila suzukii" has been composed by myself with no other resources and aid than quoted. I cofirm that the submitted thesis is my own, except where co-authored publications have been included. My contribution and contribution of the other co-authors has been appropriately and explicitly indicated. 


\title{
Dedication
}

\author{
$\mathscr{T}$ \\ My Mother, $\mathscr{M}_{y}$ Father, $\mathscr{M}_{y}$ Sibfings

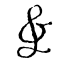 \\ My befoved Wife
}




\section{Acknowledgments}

I would like to express my sincere thanks, and gratitude to my doctor father Prof. Dr. Ernst A. Wimmer first of all for giving me the chance to pursue my doctor studies in his department under his personal supervision and guidance but also for allowing me a room of freedom to develop new ideas and strategies and implement them in my studies. His critical questions and immense knowledge were always motivational for me. I am also indebted to him for his support regarding non -academic issues. My thanks and gratitude are extended to Prof. Dr. Gregor Bucher for his help, support and fruitful discussion during the course of my study. Many thanks are due to members of the department of zoology and the department of crop protection- Universitiy of Khartoum, particulyrly Dr. Sumaia Abukashawa, for teaching me the ABCs of molecular biology. The knowledge I gained during my master studies, though to a large extent was theoratical (due to economic situation), have opened my eyes and paved my way to persue successful doctoral studies in a world reknown university.

I would like to seize this opportunity to express my gratitude to the International Atomic Energy Agency represented by Dr. Konstantinos Bourtzis for the chance to attend the meetings of the coordinated research program to improve the sterile insect technique and to the members of the CRP for fruitful discussions and suggestions. Special thanks are due to those who always support us with open heart and smile Bettina Hucke, Birgit Rossi and Merle Eggers. My thanks and appreciations also go to my colleagues and friends Kolja N. Eckermann and Mohammad KaramiNejadRanjbar for our years long work together. I would like to express my indebtedness to my colleagues Stefan Dippel and Ingrid Curril for useful molecular biology tricks. I also want to seize this opportunity to thank my colleagues and friends, Musa D. Isah and Bibi Atika for always being there when you need them and for fruitful discussions. My thanks and gratitude go to my colleagues Elisa Buchberger, Micael Reis, Amel Chtioui, for the nice talks, discussion and healthy atmosphere. Special thanks are due to my colleague Felix Quade for suggestions regarding my doctoral thesis and for his readiness to help all the time. My thanks go also to my colleague Dominik Muehlen, and Max Farnworth for discussions and exchange of ideas. I would like to thank my students Luisa Hildebrand, and Fabienne Heese for their productive work that was also crown by co-authorship. My thanks are also due to DAAD for financial support. To our technical staff at the department of developmental biology, Helma Gries, Angelika Löffers, Elke Küster, Katrin Kanbach and Claudia Hinners for their readiness and willingness to help. My thanks and gratitude are also due to Beate Preitz and Daval for their help regarding IT issues and especially Beate Preitz for introducing me to microscopy and for her continuous support in that regard.

My sincere thanks and gratitude to my first teachers ever, my mother and my father, who tought me everything to be the person I am today, their love, patience and continuous support are unmatched. To my wife, I am immensely thankful, for being there for me and for 
unconditional support, including help to print this work. Last but not least, I would like to extend my indebtedness and gratitude to my friend Mahmoud Aboud for always be there whenever I need him. 


\section{Table of Contents}

Dedication ..............................................................................................................

Acknowledgments ..................................................................... iii

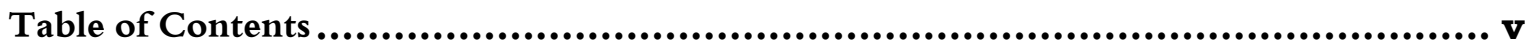

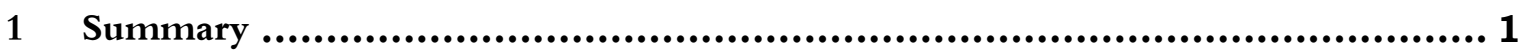

2 Introduction........................................................................ 3

2.1 The growing population of the world ...................................................................

2.2 The invasive fruit pest Drosophila suzukii ......................................................... 3

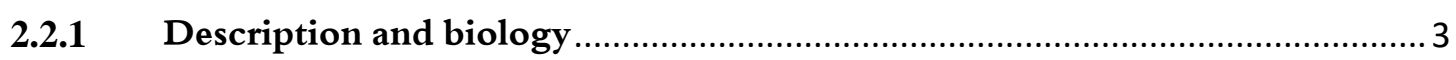

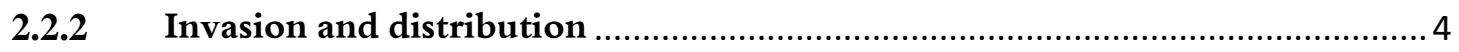

2.2.3 Damage and Economic importance ….......................................................... 4

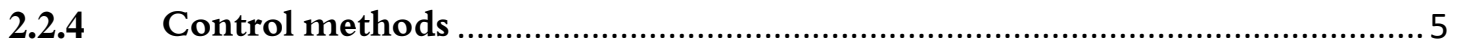

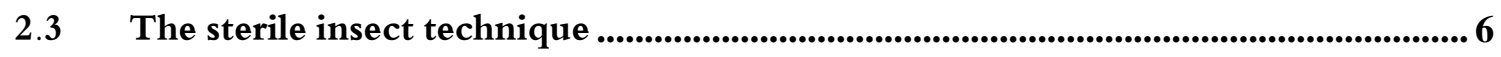

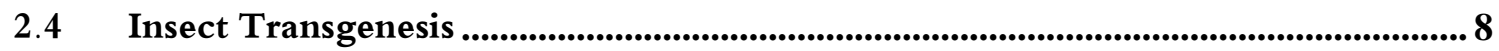

2.4.1 Random transposon-mediated germline transformation .............................. 9

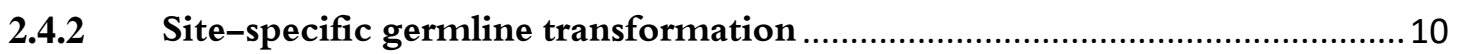

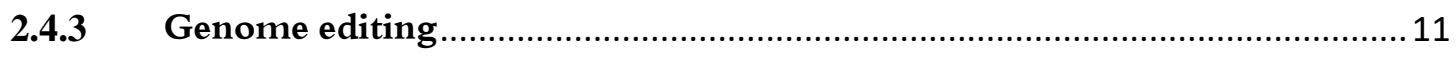

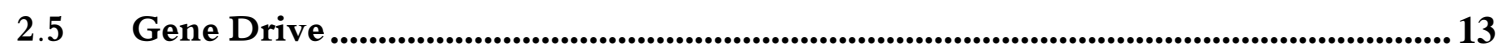

2.6 The main aims of the study ................................................................................................... 16

$2.7 \quad$ Specific objectives: .......................................................................................................................... 16

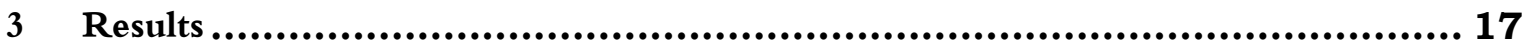

3.1 Consequences of resistance evolution in a Cas9-based sex conversionsuppression gene drive for insect pest management ............................................................19

3.2 Hyperactive piggyBac transposase improves transformation efficiency in diverse

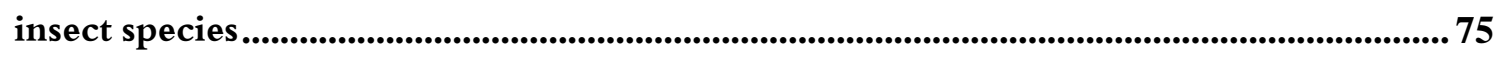

3.3 Improvement and Use of CRISPR/Cas9 to Engineer a Sperm-marking Strain for the Invasive Fruit Pest Drosophila suzukii ................................................................................ 101

3.4 Improvement on the genetic engineering of an invasive agricultural pest insect, the cherry vinegar fly, Drosophila suzukii.................................................................................. 129

3.5 Reproductive Sterility System for Drosophila suzukii control based on knockout or knock-down of specific male fertility genes. 
3.6 Perspective on the combined use of an independent transgenic sexing and a multifactorial reproductive sterility system to avoid resistance development against transgenic Sterile Insect Technique approaches

3.7 Development of a CRISPR/Cas9-induced multifactorial reproductive sterility system based on sperm-specific chromosome shredding

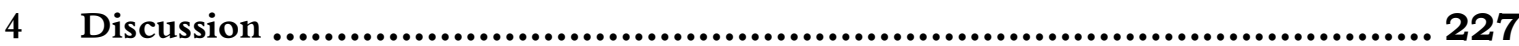

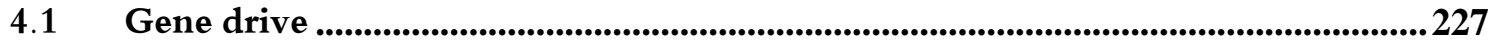

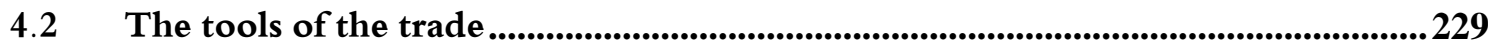

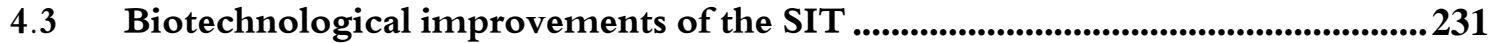

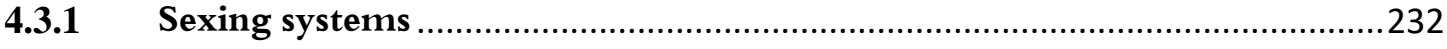

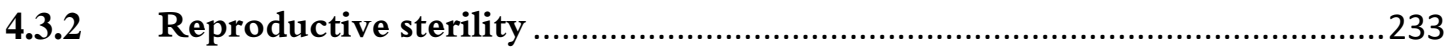

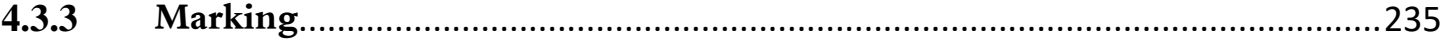

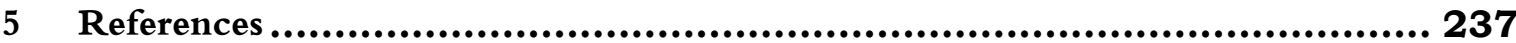

6 Curriculum Vitae ...................................................................... 253 


\section{Summary}

Globalization has contributed enormously to emergence and expansion of invasive pests. A recent example is the invasion in Europe and the USA by the cherry vinegar fly Drosophila suzukii. The pest has high potential to invade and establish itself in different climatic zones, the fact that is made clear by looking at the current global distribution map of the fly. The use of pesticide chemicals is so far the first line of defense against it due to lack of reliable alternatives. The use of pesticides against this fly has major problems including possible emergence of insecticide resistance due to the short generation time and number of generations per year. Secondly, the fly lays eggs inside the fruit which make the egg and larvae not exposed to insecticide. Thirdly and most importantly is the time of invasion with regard to ripening and harvest of the fruits. This makes the pesticide choice a very hard one. To be able to fight this onerous pest, the Sterile Insect Technique (SIT) offers an effective, environment friendly genetic pest management that does not interfere with the natural enemies and pollinators.

Here, I propose the use of biotechnological approaches to develop transgenic strains suitable for SIT against D. suzukii. In the first part of this study, the use of a CRISPR/Cas9 genome editing system to engineer a sex conversion suppression gene drive in Drosophila melanogaster is presented. We found that current designs of homing-based gene drive induce development of resistance against them. We proposed possible solutions to avoid rapid development of resistance and the application of such drive in Tephritid flies where targeting the sex determination gene transformer leads to fertile XX males. Second, the hyperactive version of the piggyBac transposase was successfully used to improve insect transgenesis with demonstration in three species belonging to two different orders, the genetic model Drosophila melanogaster, the global fruit pest Ceratitis capitata and the new beetle model for development and evolution Tribolium castaneum. In the third chapter improvement in CRISPR/Cas9 genome editing of the invasive fruit pest Drosophila suzukii is presented. Different endogenous regulatory elements were isolated and used to drive the expression of Cas9 and the guide RNAs. In addition, the application of CRISPR/Cas9 to engineer the first sperm marking strain for $D$. suzukii is presented, which represents an important contribution to the establishment of the SIT for this pest. In the fourth part, the $\varphi \mathrm{C} 31$ integrase based sitespecific germline transformation of the fruit pest $D$. suzukii was established both by integration in one attP landing site and by recombinase mediated cassette exchange (RMCE). This provides a platform for testing several enhancers and/or complete system in the same genome context. We also present the development of embryonic and spermatogenesis specific driver lines that can be used for different biotechnological improvements of SIT against $D$. suzukii. The last three chapters present a perspective paper describing a new reproductive sterility 
system and the first steps toward its development. It is based on CRISPR/Cas9 chromosome shredding during spermatogenesis that should lead to the same results commonly achieved by ionizing radiation but with no deleterious effect on the males' fitness. This should culminate in embryonic lethality due to chromosomal aberration causing aneuploidy. We first generated several spermatogenesis specific driver lines and Cas9 responder lines to test the suitability of the tet-offbinary system to control gene expression during spermatogenesis. Suitable genomic targets for chromosome shredding were identified and $g R N A$ s to target them were designed. Genome editing and insect transgenesis tools developed in this study will facilitate further biotechnological improvements of the SIT and its transfer to the invasive agricultural pest $D$. suzukii. 


\section{Introduction}

\subsection{The growing population of the world}

According to the World Population Prospects 2019 published by the Department of Economic and Social Affairs of the United Nations, the world population has increased by 2 billion since 1994 having reached 7.7 billion in mid-2019. Despite the fact that the growth rate declines, the world population is expected to continue growing to reach 8.5 billion in 2030, 9.7 billion in 2050 and 10.9 billion in 2100 (1). Concomitantly with this growth, the world is faced by global warming, which has among its impacts the increase in the number of insect pests and the severity of infestation (2). One of the major problems that faces mankind at the moment is the production of enough food to feed the growing population of the world (3). Insect pests are said to be responsible for loss of one fifth of the annual total world production of crops. Exotic or invasive pests present higher threat to food production than endogenous ones due to several factors including lack of natural enemies $(4,5)$. A prominent example is the introduction of the Asian spotted stemborer Chilo partellus into Africa (6). In a study by Groote et al. (2003) reviewed in (7) they found that the average loss in maize production in Africa oscillates between 20-40\%. Maize is considered the staple food in most of Africa. More severe damages were reported in east Africa in Ethiopia where loss in maize production reaches $100 \%$ (7) Another classical example of exotic pests though not an insect but worth mentioning is the cassava green mite. It was introduced into Africa in 1971 (8) and by the year 1985 it has spread all over the cassava belt in Africa (9). Crop loss due to infestation reach up to $80 \%$ (10). Recently an Asian fruit pest has invaded Europe and the USA and in short time become a global problem for fruits industry (11). It is obvious that global trade and transport play a major role in insect pests' invasion which necessitate strict legislation and quarantine.

\subsection{The invasive fruit pest Drosophila suzukii}

\subsubsection{Description and biology}

The dipteran fruit pest Drosophila suzukii was collected for the first time in Japan in 1916 and described later on by Shounen Matsumura as Drosophila suzukii Matsumura (12). The fly belongs to the family Drosophilidae and is commonly known as the cherry vinegar fly and in the USA termed Spotted Wing Drosophila (SWD) due to the fact that the male flies have two dark spots, one on each wing at the distal edge (13). The adult fly is $2-3 \mathrm{~mm}$ long with characteristic red eyes, a pale to yellowish brown thorax and black traverse stripes on the abdomen. The female has a prominent serrated ovipositor, which enables the fly to infest intact ripening fruits. The eggs are milky white with two dorsal appendages at the anterior. The larvae are maggot-like, white transparent with black mouthparts. There are three larval instars and the fully-grown larvae can reach up to $3.5 \mathrm{~mm}$ long. The pupae are reddish brown and have two stalks at the anterior end with finger-shaped projections. One complete cycle 
from egg to adult stage takes from a bit more than a week up to two weeks depending on the temperature (13). In California it has been found that the fly can produce up to 10 generation per year (13). During its life, a single fly has the potential to lay between 200-600 eggs with 1 to 6 eggs a day $(13,14)$

\subsubsection{Invasion and distribution}

The fruit pest $D$. suzukii invaded the Hawaiian Islands in 1980 (Kaneshiro 1983) but it took until 2008 that it has been reported in three states of the mainland USA $(12,13,15)$. In the same year, it was detected in Europe, in Italy (12) and Spain (16). Owing to its high fecundity, short generation time, and lack of regulations regarding Drosophila, the fruit pest $D$. suzukii has managed to expand its territories and invade more countries including France, Switzerland (17), Austria (18), the Netherlands (19), Germany (18), Croatia (19), the UK (20), Hungary $(21,22)$, and Turkey (23). In the Americas, the situation is not better than Europe. The fly has been detected in 41 states in the USA just four years after the first invasion in California. It has also been reported in Canada in (13). In South America the fly has established itself in Brazil (24) and recently in Argentina in four localities (25). The fly has also been reported in the middle east in Iran (26), which clearly shows the high potential of the fly to adapt to different climates.

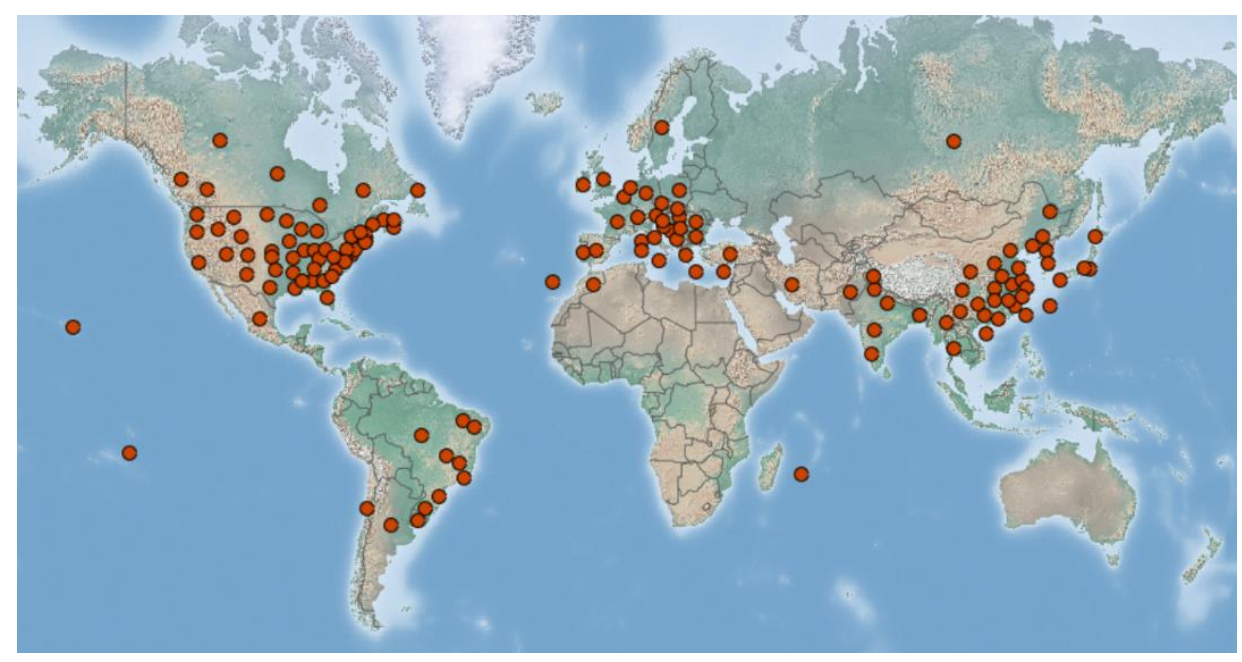

Figure. 1. World Distribution of $D$. suzukii

Source: CABI/EPPO

\subsubsection{Damage and Economic importance}

Unlike other Drosophila species, the cherry vinegar fly is armed with a prominent serrated ovipositor that enables it to lay its eggs inside intact ripening fruits at the stage of colour changing (27). The hatching larvae cause the main damage by eating the fruits from inside and rendering them unmarketable. The wounds caused by the ovipositor provide entry points for bacteria and fungi that lead to fruit rotting $(28,29)$ Fig. 2.

The fruit pest $D$. suzukii is polyphagous with a broad host range spanning wild fruit plants as well as cultivated crops and non-crop ornamental plants $(30,31)$ which exacerbate the 
situation by serving as refuge for the fly during the off-season. Among the affected crops are, blueberry, strawberry, currants, raspberry, blackberry, plums, grapes, as well as stone fruits such as cherries and apricots [lee et al 2015, 19-25] (30,31). A study conducted by Bolda et al (2010) (15) to assess the magnitude of the economic impact of D. suzukii infestation in strawberry, blueberry, blackberry, raspberry, and cherry in three US states (California, Oregon and Washington) revealed revenue loss of more than 500 million USD (15). This study however, didn't consider price adjustment due to reduced supply of fruits. The impact of $D$. suzukii in small fruit production in Trentino (Italy) in term of revenue with regard to the five fruit crops strawberry, raspberry, blueberry, blackberry, and cherry was more than 3.3 million Euro (32).

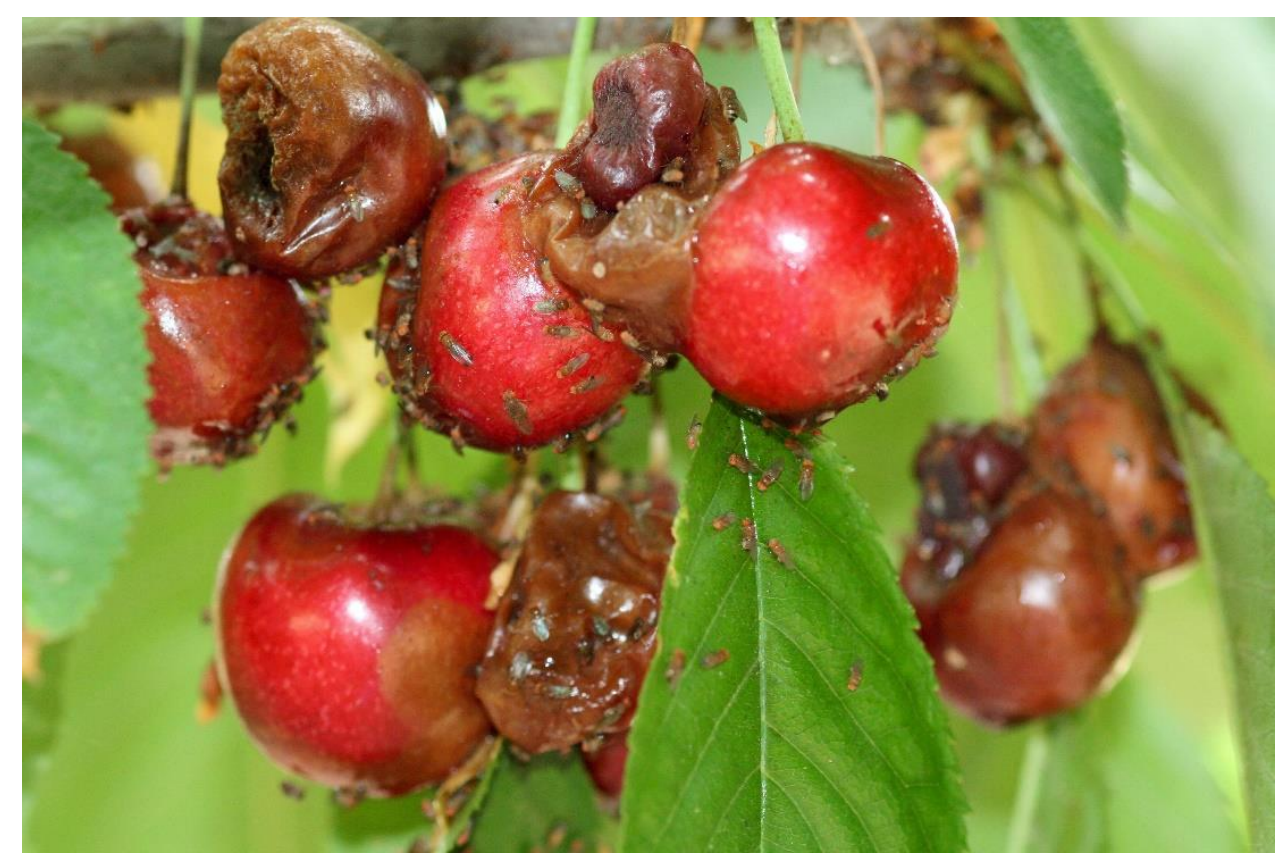

Figure. 2. Consequences of $D$. suzukii infestation on cherry

"Copyright (C) 2016 Regents of the University of California. Used by permission."

\subsubsection{Control methods}

Insect pests can be controlled by different strategies such as cultural control, chemical control, biological control, or genetic control and a combination thereof applied in the integrated pest management (IPM) strategy. To control the invasive fruit pest $D$. suzukii, growers have tried different strategies. Cost-benefit studies and estimations of revenue losses due to infestations by $D$. suzukii in selected fruit crops gave strong indication that the benefit gained from management overweigh the costs of crop loss when no control measures are deployed $(33,34)$. The use of nets around the crop to prevent infestation of the fruits by the fly proved to be very effective but has high initial cost and is suitable only for small areas (34). Currently most efficient method for the control of the invasive pest $D$. suzukii are pesticide chemicals. Among the groups of insecticides that are tested against the cherry vinegar fly are spinosyns, 
organophosphates, pyrethroids, and neonicotinoids $(35,36)$. In a broad screen for effective insecticides, Bruck et al. (2011) (35) tested insecticides belonging to different groups. Effective control against adult $D$. suzukii was obtained by direct application of pyrethroids (bifenthrin, beta-cyfluthrin, permethrin, zeta-cypermethrin), organophosphates (malathion, diazinon), and spinosyns (Spinosad, spinetoram). Spinosad efficiency was later on confirmed in another screen by Cuthbertson et al. (2014) (37).

\subsection{The sterile insect technique}

The recent years have witnessed increase in the awareness of the adverse effect of chemical control of insect to the environment and the human health. The fact that urged the need for alternative pest control approaches that are environment friendly and ideally species specific. One approach to address this need is the sterile insect technique (SIT), the first genetic control strategy in which reproductively sterile males of the target pest are released to introduce reproductive sterility on the wild population by competition with the wild type fertile males over the wild type fertile females leading to infertile mating and thus population suppression (38). The idea of releasing pest insects to supress the pest population in the field by introduction of reproductive sterility was conceived in the 1930s - 1940s by three independent scientist. Knippling based on his observations that the New World Screwworm mates only once (monogamous), he proposed, if large numbers of the insect can be produced and if the males can be rendered sterile and released in the target area, the local population of the pest will be suppressed (38). He also proposed that, if the treatment area is isolated, successive releases of the sterile insects can result in complete eradication of the pest. Bushland (1960) reviewed in (39) added that even polygamous insect pests can be controlled by means of SIT providing that the sperm produced by the sterile males is used to fertilize the egg (40).

The SIT on practice today encompasses mass production of the target pest or vector, removal of females, sterilization of the males by ionizing radiation, marking, and sustained inundative release of the sterile males in the target area to compete with their wild type counterparts over the wild type females leading to infertile mating and thus population suppression $(38,40)$. Now the SIT is considered an important component of Area-Wide Integrated Pest Management Programs (41). The first and most successful example of integration of SIT into AW_IPM programs was launched in the 1950s against the New World Screwworm in the south eastern USA, where decade long efforts were crowned by the eradication of the insect from USA, Mexico, and Central American including Panama (42). The same approach has also been deployed to eradicate the new world screw warm from North Africa, Libya (43) 


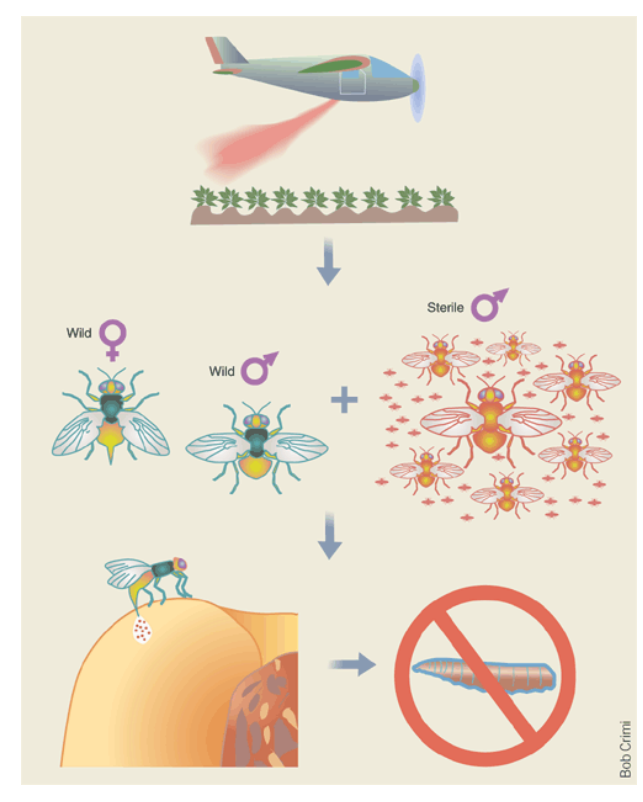

Figure. 3. Illustration of the SIT

Source (Wimmer, 2005)

To be able to establish an SIT program against a particular insect pest, a method of mass rearing should be available. Importantly a method for sex separation (sexing) that ensures production of fit males only is required. Release of female insects even if they are sterile is not accepted especially for insect vectors of disease because sterile females can still transmit the disease (44). Also release of females of some agricultural pests is not accepted, e.g. Tephritid fruit flies have ovipositors by which they pierce and lay their eggs inside intact fruits and damage them (44). Furthermore, release of big numbers of females along with the sterile males compromise the efficiency of the system since the released females will act as competitive mates for the sterile males (45).

So far, the best and most efficient sexing strain develop and deployed worldwide in operational Area wide IPM programs is the so-called Vienna 8 genetic sexing strain (GSS) strain of the Mediterranean fruit fly, Ceratitis capitata (46). The strain is based on temperature sensitive lethal ( $t s h$ allele and white pupa marker linked together on an autosome and a translocation of the wild type genomic region onto the Y chromosome (47). This allows removal of females during embryonic development by exposing the eggs to $34^{\circ} \mathrm{C}$ for $24 \mathrm{~h}(48,49)$. The stability of the strain and the efficiency of sexing can be regularly checked, since the females develop white pupae whereas males produce wild type brown pupae. Unfortunately, such strains cannot be directly copied in other insect pests and the whole procedure used for generation of Vienna 8 has to be repeated for each new pest species in a hope that a tsl allele can be obtained, a wild type copy be translocated to the male sex chromosome, and a linked visible marker be identified for ease of checking. A generic approach for generation of sexing strains so far developed is the transgenic female-specific embryonic lethality which has so far been developed for several insects $(50-52)$. Sexing has also been achieved based on natural variation 
between males and females for example in the size of the pupae $(53,54)$ or based on biology such as the difference of the time of emergence of the males and females (Tse-tse flies) (55).

So far, sterilization of males to be released is achieved by ionizing radiation of the pupae. Irradiation induces chromosomal breaks, which lead to reproductive sterility due to chromosomal aberrations causing aneuploidy in the progeny (56). One important feature of ionizing radiation is redundancy in the cause of sterility, which decreases the probability of resistance development (56). However, the use of radiation affects the fitness of the males compared to their wildtype counterparts and therefore males have to be released in big numbers to compete with the wild types for the females. One of the biotechnological improvements of the SIT with regard to sterilization is the conditional transgenic embryonic lethality system which has first been tested in Drosophila melanogaster and later on developed for several agricultural pests $(57,58)$. The conditionality of the system is achieved by using the tet-off binary system $(59,60)$, whereby an early embryonic promoter is used to drive the expression of a heterologous transcription factor the tetracycline-controlled transactivator (tTA) $(57,58)$, which in turn activates an effector such as a proapoptotic gene that is placed downstream of the $t T A$ responsive element (TRE). A double homozygous strain of the two transgenes must be reared in food supplemented with tetracycline (tet) or an analogue to suppress the lethality when tet binds tTA to prevent its binding to the TRE and the effector is off. Reproductive sterility has also been induced by the development of a system based on conditional establishment of a positive feedback loop of expression of the tTA leading to a cytotoxic effect. The system is referred to as the release of insects carrying a dominant lethal (RIDL). (61)

It is beneficial to mark the males before release to be able to track and monitor the dynamic and success of the SIT program. So far this has been done using fluorescent dusts to dust the pupae before release and when the males emerge, they get some of that dust on their bodies. This however, beside its bad impact on the health of the facility workers it is also not very efficient. Improvement in this regard was also been done using a transgenic approach, in which a spermatogenesis-specific promoter is used to drive the expression of a fluorescent protein such as the green fluorescent protein EGFP or the red fluorescent protein DsRed (62-65). The system has the advantage over the classical way, that it allows also tracking of the success of the program by random collection of females and examination of their spermatheca (sperm storage organ) for the presence of marked sperm transferred from the released sterile males.

\subsection{Insect Transgenesis}

The term transgenesis refers to the introduction of foreign DNA into the genome of an organism (66). The ability to generate transgenic animals has contributed tremendously to our understanding of the mechanisms of development and gene regulatory networks (67-69). Transgenic animals have been used over the years in biomedical research to study human 
diseases, disease associated alleles, and cancer as well as in basic research to understand diverse biological processes.

To be able to generate transgenic animals, three components have to be secured, first a suitable gene vector to allow integration of the gene of interest in the genome of the organism, secondly, a method to deliver the vector to the primordial germline, and thirdly, a marker to facilitate screening for the transformants (66). Different vectors have been developed to suit the particular need including virus and transposon-based vectors, site specific recombinases, and endonucleases. Insect germline transformation is routinely done using transposon-based vectors with the first demonstration in the genetics model Drosophila melanogaster using the P-element (68)

At that time transformation markers were based on rescue of visible but viable recessive mutant phenotypes such as the use of mini- white gene to rescue the white eye phenotype in the flies (70). The discovery of fluorescence proteins enabled the development of different transformation markers even for non-model insects. The Drosophila promoter of the Ubiquitingene fused to EGFP was found to drive strong ubiquitous expression in Drosophila (71). It has also been used in the medfly Ceratitis capitata. The viral promoter of the baculovirus immediate early gene $i e 1$ has also been used alone and in combination of the baculovirus enhancer sequence $h r 1$ to drive strong expression of fluorescent proteins $(72,73)$. The best example of a versatile transformation marker is based on the synthetic eye-specific promoter 3 XP3 based on the Pax-6 binding site and the basal promoter of the Dm hsp 70 gene (67). It has been used to drive the expression of the fluorescence protein in the eyes of many different insect species (74-77)

\subsubsection{Random transposon-mediated germline transformation}

Transposable elements or jumping genes were described originally in the late 1940s by McClintock, when she was studying chromosomal breakage in maize and her findings were published in 1950 in a PNAS article entitled 'The origin and behaviour of mutable loci in maize' (78). The discovery of the potential of using transposable elements as gene vectors to stably integrate a transgene into the chromosome of the target organism has revolutionized the field of genetics and allowed detailed studies in functional genetics and genome structure including gene disruption, deletions, translocations etc. Type II DNA transposons are the most commonly used mobile genetic elements in genetic engineering (66). They move between host chromosomes through a 'cut and paste' mechanism facilitated by an enzyme called transposase encoded within the transposable element (79). To use a transposon as a gene vector, plasmids are engineered in a way that the gene of interest is flanked by the transposon terminal inverted repeats (TIRs) and the transposase necessary for transposition is provided in trans commonly from a helper plasmid, on which the transposase coding sequence is fused to a constitutive or inducible promoter such as the heat shock promoter (66). 
The Drosophila specific P-element, is unfortunately limited in functionality to Drosophila and closely related species due to the requirement of host-specific factors (80). The most commonly used transposons in insect germline transformation belong to three super-families, the Tc/mariner, the piggyBac, and the hAT superfamily. Members of Tc/marinerare the most abundant transposons and distributed among different taxa from plants to mammals (81). Mos1 was discovered in $D$. mauritiana and was used as gene vector in several systems $(82,83)$. Another example of Tc/mariner is the Minostransposon from D. hydei. The hAT superfamily is represented by the Hermes transposon from the house fly Musca domestica. The best characterized and widely used transposon in genetic engineering is the piggyBac vector from the piggyBac superfamily. The element was originally identified from a mutant baculovirus strain in a cell culture of the cabbage looper Trichoplusia ni (84). Vectors based on piggyBac were used to transform many model and non-model organisms. The efficiency of piggyBac germline transformation can be improved by the use of mutant hyperactive variants of the transposase enzyme (85).

\subsubsection{Site-specific germline transformation}

Besides transgenesis by random transposon-based integration, scientists have developed sitespecific strategies that allow integration of the transgene into a predefined and characterized docking site. This facilitates testing different transgenes or enhancers at the same chromosomal environment and thus avoid variation in expression due to position effects. The most commonly used systems are the site-specific recombinase systems such as Cre/lox, flp/FRT and the $\varphi \mathrm{C} 31$ integrase system (86-88). In all cases, recombination takes place between a sequence in the genome usually introduced by random transgenesis and a sequence in a donor plasmid along with the transgene to be integrated. The Cre (causes recombination) or cyclization recombinase is a tyrosine site-specific recombinase of $38 \mathrm{KD}$ discovered in the bacteriophage P1 (89). The enzyme recognizes short DNA sequences (lox $P$ ) and mediates recombination between two identical lox sites (89). Based on the orientation of the two sites, the recombination event results in deletion, inversion or translocation (90-92). The system has been widely used to manipulate transgenic mice, to drive tissue specific ectopic expression, or tissue specific knockdown. In insects, it has been used as site-specific germline transformation tool, as well as for functional genetic studies by ectopic tissue specific expression of genes or by tissue specific knockout. Another example of a tyrosine site-specific recombinase is the Flp/FR T system of the $2 \mu$ plasmid which is similar to Cre/lox, as the flipase enzyme mediates recombination between two identical flipase recognition targets ( $F R T)$. The system has been extensively used in insects. Especially in Drosophila $(87,93,94)$ it has also been used as a tool for site-specific germline transformation only by RMCE due to reversibility of the recombination reaction and the kinetic barrier that favour the excision, the two system Flp/FRT and Cre/lox function only by RMCE (95). 
A third but distinct example of site-specific recombinases is the $\varphi \mathrm{C} 31$ integrase which is derived from the bacteriophage $\varphi \mathrm{C} 31(96)$. It is a serine site-specific recombinase that mediates recombination between two different DNA sequences called attachment sites ( $a t t$ ) one in the bacteriophage genome (attP) that facilitates its integration into the bacterial chromosome by recombination with the bacterial attachment site $(a t t B)(97)$. The advantage of this system over the Cre/lox and the Flp/FRT systems is that the recombination is unidirectional and therefore the integration is stable (98). This system was used in many model and non-model insects as a tool for site-specific germline transformation by simple integration or by recombinase mediated cassette exchange (99). In the later, case two reciprocal recombination events occur between two attPsites integrated in the genome and two attBsites in the donor plasmid.

\subsubsection{Genome editing}

The ability to manipulate the genome of organisms is of paramount importance both in basic as well as in applied research. especially in the field of gene therapy and biotechnology. Traditionally, chemical and transposon-mediated random mutagenesis was used in forward genetics to induce mutations in the genome followed by intensive screening for phenotypes of interest and more work to identify the molecular basis of the phenotype (100-103). These approaches, despite being time consuming and labour intensive, have served the community for many years in the pre-genomic era. One of the drawbacks of these methods is the lack of specificity which is often accompanied by undesired changes in the genome. In recent years, with next generation sequencing being affordable and the genome of many non-model organisms being sequenced, huge genomic data are being generated and made available to researchers through public databases. To study the function of these genomic information, tools for reverse genetics are required especially for targeted gene mutagenesis. Homologous recombination-based gene targeting has been used, however, with very low efficiency (104). Two discoveries have prompted the development of site-specific genome editing tools: (i) The discovery that a double strand break (DSB) in the targeted gene increases homologous recombination by at least 3 orders of magnitude $(105,106)$; and (ii) that in the absence of a homologous repair template, the DSB is repaired by the error-prone Non-Homologous End Joining (NHEJ) mechanism (107).

In functional genetic analysis, the induction of a site specific DSB in the gene of interest (GOI) is enough to obtain loss-of-function mutations, since the repair by NHEJ leads to insertions or deletions (indels) of a few nucleotides, which result in frameshifts and/or premature stop codons (107). It is important in this approach to target close to the translation start codon, however, not too close, since an alternative translation start may be used. Alternatively, a repair template may be provided that has the modification of interest flanked by homology arms to facilitate homology dependent repair (HDR) of the DSB (108). The later approach allows precise modification to be introduced down to the resolution of a single nucleotide 
exchange. Among the endonucleases that were used until recently are the rear cutting restriction endonucleases such as the yeast meganuclease I-SceI (109). More recently, synthetic zinc finger nucleases have gained popularity among scientists and were adopted for many systems including many insects (110). In 2011, it has been chosen by Nature Methods as the "Method of the Year". It is a programable endonuclease generated by fusion of ZincFinger DNA binding domains to the catalytic domain of the restriction enzyme FokI (110). The TALENs, are another programable endonucleases based on the transcription activatorlike effector TALE, a protein that is secreted by the plant pathogenic bacteria of the genus Xanthomonas during infection (111). They are engineered by fusion of the TAL effector DNA binding domain to the cleave domain of the restriction enzyme FokI (111). One of the advantages of TALENs over ZFNs is that it can be programmed to target any part of the genome (112). The discovery of the bacterial adaptive immune system $(113,114)$ called CRISPR/Cas9 and its adoption as a tool for genome engineering has opened many doors that were not possible otherwise (115). The main advantage of this system over TALENs and ZFN, is the very low cost and the ease of programming of Cas9 to target any part of the genome (115). The system consists of the Cas9 endonuclease and two small RNAs called CRISPR$R N A$ ( $r R N A)$ which dictates the specificity of Cas9 by means of 17-20 nucleotides, and trans-activator RNA (tracrR $N A$ ) which is involved in processing and maturation of crRNA and facilitates the interaction of crRNA with Cas9 (116). Scientists have engineering the two small RNAs into one chimeric guide RNA ( $g R N A$ ) to simplify the delivery (117). A very important feature of Cas 9 target sites is the PAM sequence, an acronym for Protospacer Adjacent Motif(118). This sequence varies for different Cas9 enzymes. However, for the most commonly used version, $S p C a s 9$, which was isolated from the bacteria Streptococcus pyogenes, the PAM is NGG where $\mathrm{N}$ is any of the four different nucleotides (118). The crystal structure of the Cas9 complex with $g R N A$ and a PAM-containing DNA target revealed that the PAM sequence remains in a base-paired duplex. The PAM GG in the target strand (noncomplementary) is scanned via the major groove by a conserved arginine residue in the PAM interacting domain of Cas9 at the C-terminus. Interaction of the PAM-Domain with the PAM through the minor groove put the target DNA strand in the right orientation for base pairing. This recognition and interaction lead to local strand destabilization and un-winding. This clearly explains the importance of the PAM sequence (118).

The CRISPR/Cas9 system has been rapidly adopted and established for many organisms from yeast to human as a tool for genome editing, functional genetic analysis by gene knockout and targeted mutagenesis $(117,119-121)$ or by activation and repression of gene expression (122125 ) and even modification of epigenetic imprinting by methylation or demethylation $(126,127)$

To achieve all the mentioned functions, the Cas9 molecule itself has been engineered. For example, a dead Cas9 (dCas9) that can still recognize and bind the genomic target was 
engineered by two amino acids substitutions D10A and H840A inactivating the two nuclease domains RuvC and $\mathrm{HNH}$, respectively $(122,125)$. This dCas9 was then utilized to create heterologous transcription factors (TF) by fusion of activation domains from natural transcription factors to the $\mathrm{N}$ or $\mathrm{C}$-terminus of dCas 9 and thereby creating a programable $\mathrm{TF}$ that can be targeted to enhancers of genes of interest $(122,124,125)$. It has also been engineered by fusion of enzymatic effector domains to dCas 9 to induce targeted epigenetic reprograming for example methylation, demethylation, or histone acetylation (128). Furthermore, a nickase Cas9 (nCas9) was generated with only one nuclease domain inactivated. This has been exploited for genome editing by homology directed repair, targeted mutagenesis, and importantly as a scaffold to fuse more functional domains (129-131). With first demonstration in yeast, cultured mammalian cells, and plants, Cas9-deaminase was generated by fusion of cytidine deaminases such as APOBEC1 or AID to nCas9 or dCas9 (132-136) and it has been shown to efficiently substitute a $\mathrm{C}>\mathrm{T}$ at the target site.

In applied research using CRISPR/Cas in gene therapy, the search for more smaller and highly precise versions of Cas9 is ongoing. Another CRISPR/Cas system that is gaining popularity is Cas12a due to its slightly smaller size, different PAM requirement and the ability to process an array of $g R N A s$ from a single transcript without the need for extra factors $(137,137)$. In applied insect biotechnology the system has been used or proposed to be used to develop new pest control strategies such as gene drive (138-140), and X chromosome shredder (141).

\subsection{Gene Drive}

Some naturally occurring genes tend to be inherited in a super-mendelian fashion, which means more often than is predicted by Mendelian segregation $(142,143)$. Such genes are referred to collectively as selfish genetic elements. Homing Endonuclease Genes (HEG) are selfish genetic elements that reside in the chromosome of their host organism and encode an enzyme, which recognizes and cleaves a sequence in the sister chromosome and thus get themselves copied by the cell DNA repair machinery, namely by homology dependent repair in a process called homing (144). The idea has incited scientist to attempt to engineer these genes into 'gene drive' to manipulate natural population of insect pests and diseases vectors by introducing desired traits or fitness costs. Austin Burt suggested three criteria to be met for an engineered HEG to be used for pest control: (i) HEGs should be engineered in a way that they recognize and cut a sequence in an essential gene and get copied in the middle of its recognition sequence and thus disrupt the function of the gene. (ii) The knockout of the target gene should be recessive with minimal phenotypic effect in the heterozygous situation and deleterious in the homozygous individuals. (iii) The engineered HEG is driven by a meiosisspecific promoter to ensure normal development of the heterozygous zygote that will transmit the HEG in a biased fashion to its gametes (145). 
The discovery of the CRISPR/Cas9 system as a programmable tool to introduce site-specific DSBs in the genome opened the door for scientists to realize the long-standing dream of engineering complete natural populations of insects of agricultural and medical importance. The advantage of this system over natural HEG is the ease of programming Cas 9 to target any part of the genome simply by designing $20 \mathrm{bp}$ of the $g R N A(115)$. The repertoire of genomic targets is increasing by the discovery of new Cas 9 systems such as $C p f 1(137,146)$ and by the engineering of the old ones $(147,148)$. The first demonstration that CRISPR/Cas9 can be used to transform heterozygous mutations into homozygous with high efficiency in what is called the mutagenic chain reaction (MCR) has put the cornerstone for the first CRISPR/Cas9-based gene drive (140). The simplest CRISPR/Cas-based gene drive cassette consists of the $\operatorname{Cas} 9$ endonuclease coding sequence under a meiosis-specific promoter, and the custom $g R N A$ driven by a promoter of an $R N A$ polIII gene such as the $U 6$ small nuclear $R N A$ gene flanked by flaking sequences of the $g R N A$-targeted sequence A few months after the MCR was published, the same group has reported on the use of CRISPR Cas 9 to engineer gene drive for manipulation of the malaria mosquitoes Anopheles stephensi(138). Their results showed that for such a gene drive to function its activity has to be restricted to the germline otherwise, many mutant alleles lacking the drive can form. A second study demonstrated the feasibility of using CRISPRCas9 to build suppression gene drive by targeting three female fertility genes with homozygous sterile phenotype (139). Those two studies were then followed by several attempts to address issue such as containment of the drive and improvement of CRISPR/Cas9 design. A simple homing-based gene drive that doesn't impose fitness cost on the carrier should presumably be able to take over the whole population over many generations Fig. 4. 


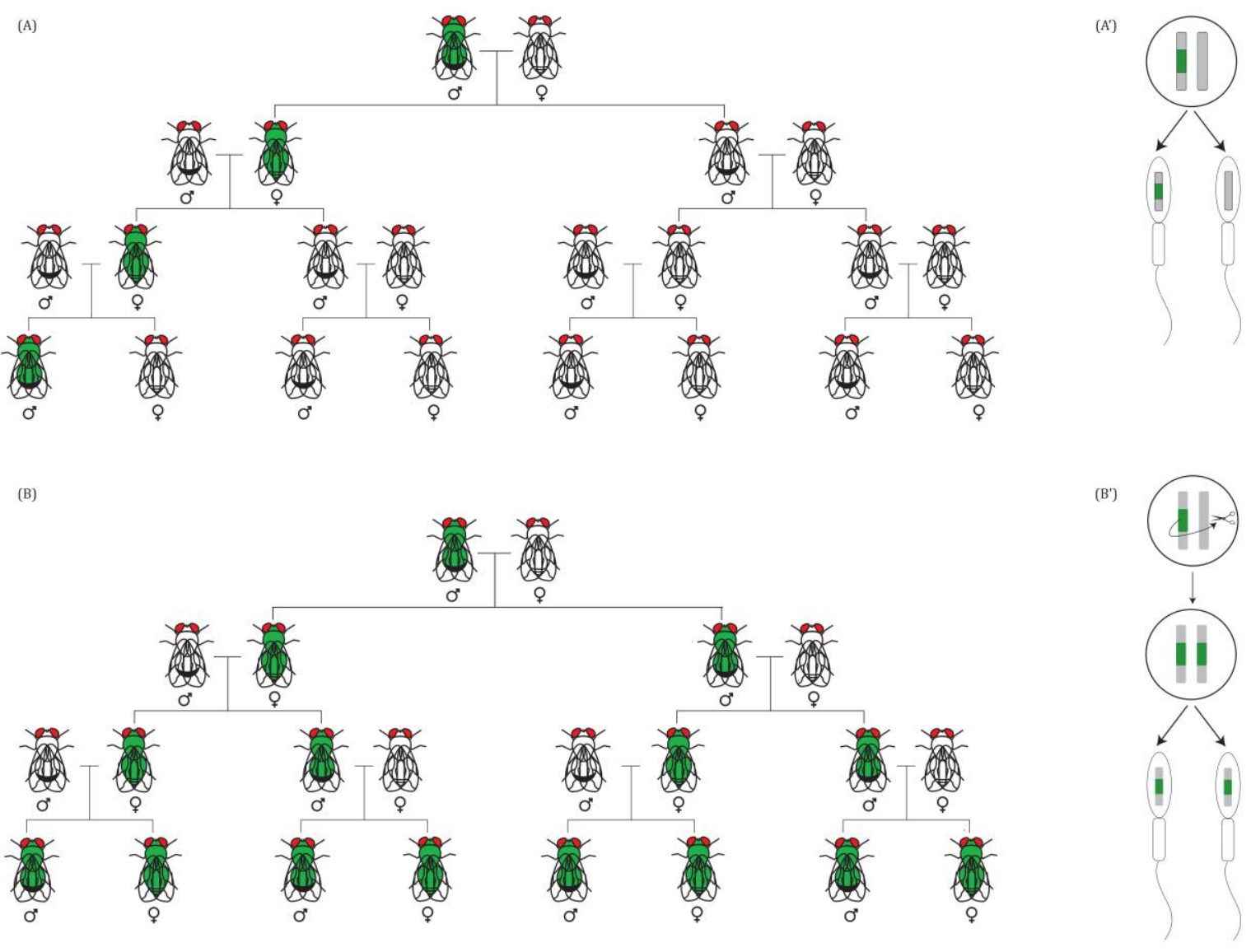

Figure. 4. Mendelian inheritance versus hypothetical gene drive. (A) Show Mendelian inheritance of a single dominant allele of a gene that produce green flies, abbreviated hereinafter as ' $g r$ '. Starting with a heterozygous male for $g r$, and assuming no advantage or fitness cost associated with this allele, half of the progeny will inherit $g r$. If only a few alleles of $g r$ are introduced into the population, over the time the allele will be diluted and lost. (A') A simplified depiction of the cytogenetic basis of the observed ratio. During spermatogenesis, half of the sperm produced should carry the $g r$ allele whereas the other half not. (B) if $g r$ is linked to a homing-based gene drive, and no fitness cost is associated with the drive cassette, all the progeny from a heterozygous male will develop into green flies and the process will continue until the drive takes over the population. ( $\mathrm{B}^{\prime}$ ) explains the homing process, the drive cassette contains a homing endonuclease gene driven by a male meiosis promoter and is placed in the middle of its target sequence. Starting with a heterozygous male, during meiosis, the HEG will target the homologous chromosome, induce DSB and the cell copies it to that chromosome creating a homozygous situation. This should theoretically lead to $100 \%$ of the sperm producedcarry the grallele. 


\subsection{The main aims of the study}

I. Explore the possibility of using Cas9-based gene drive for population suppression and possible challenges and pitfalls.

II. Establish and/or improve transgenesis and genome editing tools for D. suzukii.

III. Develop new reproductive sterility systems to establish the SIT against D. suzukii

\subsection{Specific objectives:}

The emergence of CRISPR/Cas9 as a genome editing tool has provoked scientists to build Cas9-based synthetic gene drives as pests' and vectors' control approach. However, we still don't know the suitability of Cas9 to build such systems and the problems that might arise. To address that we set the following specific objectives forth:

i. Generation of Cas9-based gene drive targeting D. melanogaster transformer (tra) gene

ii. Study the dynamic of the drive over several generations.

iii. Study the effect of emergence of resistance alleles on the drive efficiency.

The second aim of the study can be broken down into the following specific objectives:

i. Improvement in piggyBac germline transformation.

ii. Improvement of CRISPR/Cas9 genome editing system in D suzukii.

iii. Establishment of $\varphi \mathrm{C} 31$ site-specific recombination and RMCE in D suzukii.

iv. Generation of sperm-marking system for $D$. suzukii.

The third aim is to develop conditional reproductive sterility systems based on CRISPR/Cas9 or CRISPR/Cas9 and RNA interference. The first system relies on Cas9 and RNAi to target and knockout/down spermatogenesis-specific paternal effect genes involved in fertilization, which should lead to production of viable males that produce sperm and transfer it successfully to the females, and the sperm should be able to enter the egg but should fail to initiate embryogenesis. Specifically, the following will be done:

i. Identification and validation of CRISPR targets in the paternal effect gene sneaky (snky)

ii. Design of $s h R N A s$ to target and knockdown Dm snky.

The second system relies on Cas9 targeting abundant sequences in the genome of the fly leading to chromosome shredding during spermatogenesis. This should lead to embryonic lethality due to aneuploidy in the embryo. This is similar to the action of radiation but specific to spermatogenesis. As a proof-of-concept we aim to establish the system in D. melanogaster.

i. Identification of abundant CRISPR-targets in D. melanogaster transposable elements

ii. Use of the tet-off system during spermatogenesis. 


\section{Results}

Results are presented in seven chapters $3.1-3.7$. Each chapter is a manuscript which is either published, submitted or work is on going. Each of which is preceded by a one-page describing the following:

- The main objective of the manuscript in the context of the whole thesis.

- Contribution of the authors in the practical work.

- The status of the manuscript.

References cited in each manuscript are included at the end of the respective chapter.

References cited in the general introduction and discussion are at the end of the thesis in chapter 5 


\subsection{Consequences of resistance evolution in a Cas9-based sex conversion- suppression gene drive for insect pest management}

This chapter discusses the use of a Cas9-based synthetic gene drive for insect population suppression and the observed rapid emergence of resistance. The vinegar fly $D$. melanogaster offers a safe model.to study the emergence and dynamics of resistance development against a sex conversion gene drive, since sex conversion in Drosophila by disruption of the female sex determination pathway leads to the production of sterile intersexes due to the discordance between the entity of the soma and the karyotype of the germline. This therefore presents a barrier against an unintentional introgression of the gene drive cassette into a wild population. We further go to discuss and provide a model for the use of such a system in the cosmopolitan fruit pest Ceratitis capitata in which concordance between the somatic cell identity and that of the germline is not necessary. Therefore, gene drive targeting the transformergene, which is responsible for the establishment of the program to produce females, leads to production of viable and fertile XX males. This chapter thus presents an important advancement in understanding CRISPR/Cas9-based homing element gene drives as well as their problems in the application for the control of agricultural pests and proposes a solution to avoid development of resistance.

Mohammad KaramiNejadRanjbar ${ }^{\perp}$, Kolja N. Eckermann ${ }^{\perp}$, Hassan M. M. Ahmed ${ }^{\triangle}$, Héctor M. Sánchez C., Stefan Dippel, John M. Marshall, Ernst A. Wimmer

$\Delta=$ Co-first authors

Authors contributions as stated in the published paper:

M.K., H.M.S.C., S.D., J.M.M., and E.A.W. designed research; M.K., K.N.E., H.M.M.A., H.M.S.C., and J.M.M. performed research; M.K., K.N.E., H.M.M.A., H.M.S.C., S.D., J.M.M., and E.A.W. analyzed data; M.K., K.N.E., J.M.M., and E.A.W. wrote the paper; and H.M.S.C. and J.M.M. designed and analyzed the population dynamic models.

My specific contributions were particularly to Fig. S1D, Fig. S3, and Fig. S5 leading to main Fig. 2.

Status of manuscript: Published in Proceedings of the National Academy of Sciences (PNAS) in 2018. DOI: $\underline{10.1073 / \text { pnas.1713825115 }}$ 


\title{
Consequences of resistance evolution in a Cas9-based sex conversion-suppression gene drive for insect pest management
}

\author{
Mohammad KaramiNejadRanjbar ${ }^{\mathrm{a}, 1,2}$, Kolja N. Eckermanna ${ }^{\mathrm{a}, \mathrm{b}, \mathrm{c}, 1}$, Hassan M. M. Ahmed ${ }^{\mathrm{a}, 1}$, Héctor M. \\ Sánchez ${ }^{\mathrm{C}}$ d, Stefan Dippel ${ }^{\mathrm{a}}$, John M. Marshall ${ }^{\mathrm{d}}$, Ernst A. Wimmer ${ }^{\mathrm{a}, \mathrm{b}, 3}$

\begin{abstract}
a Department of Developmental Biology, Johann-Friedrich-Blumenbach-Institute of Zoology and Anthropology, Göttingen Center of Molecular Biosciences, University of Göttingen, 37077 Göttingen, Germany. ${ }^{b}$ Göttingen Graduate Center for Neurosciences, Biophysics, and Molecular Biosciences, University of Göttingen, 37077 Göttingen, Germany. ${ }^{c}$ Molecular Cell Dynamics, Max-Planck-Institute for Biophysical Chemistry, 37077 Göttingen, Germany. ${ }^{d}$ Divisions of Biostatistics and Epidemiology, School of Public Health, University of California, Berkeley, CA 94720 USA.
\end{abstract}

Edited by Allan C. Spradling, Carnegie Institution of Science, Baltimore, MD, and approved May 9, 2018 (received for review August 7, 2017).

The use of a site-specific homing-based gene drive for insect pest control has long been discussed, but the easy design of such systems has become possible only with the recent establishment of CRISPR/Cas9 technology. In this respect, novel targets for insect pest management are provided by new discoveries regarding sex determination. Here, we present a model for a suppression gene drive designed to cause an all-male population collapse in an agricultural pest insect. To evaluate the molecular details of such a sex conversion-based suppression gene drive experimentally, we implemented this strategy in Drosophila melanogaster to serve as a safe model organism. We generated a Cas9-based homing genedrive element targeting the transformergene and showed its high efficiency for sex conversion from females to males. However, non-homologous end joining increased the rate of mutagenesis at the target site, which resulted in the emergence of drive-resistant alleles and therefore curbed the gene drive. This confirms previous studies that simple homing CRISPR/Cas9 gene-drive designs will be ineffective. Nevertheless, by performing population dynamics simulations using the parameters we obtained in D. melanogaster and by adjusting the model for the agricultural pest Ceratitis capitata, we were able to identify adequate modifications that could be successfully applied for the management of wild Mediterranean fruit fly populations using our proposed sex conversion-based suppressiongene-drive strategy. 


\section{Background}

The use of CRISPR-Cas9 systems as a homing-based gene-drive tool to alter the genotype of insect populations has theoretically (1-5) and practically (6-8) been shown to be feasible. These systems can potentially allow the spread of any desired trait in a wild population of target species even if the desired phenotype imposes a fitness cost $(2,4,5,8)$. Therefore, the spread of lethality or sterility traits that could result in suppression and eventually collapse of the target population should be possible. This has recently attracted special attention in pest and disease vector control $(1,3,6-8)$. However, the effort had focused mainly on disease-vector mosquitoes such as Anopheles $(7,8)$. In homing CRISPR/Cas9 gene-drive (HCGD) systems, a CRISPR/Cas9 homing element (CHE) composed of at least the Cas9 endonuclease-coding sequence and a guide RNA (gRNA) is integrated in the host genome at the gRNA target site. In the heterozygous state, Cas9 introduces an RNA-guided double-strand break in the wildtype allele (similar to homing endonucleases) which then will be repaired either by homologydirected repair (HDR) or error-prone mechanisms such as non-homologous end joining (NHEJ). In the former case, the CHE allele serves as the repair template and is copied into the homologous chromosome. Directing this process to the germline will result in superMendelian inheritance driving the CHE and any accompanying genes into the population. Therefore, the highly customizable nature of CRISPR/Cas9 allows simple design of HCGDs to drive any desired trait, even those resulting in sterility, into wild populations as long as the cost of this phenotype does not surpass a certain threshold $(1,4)$.

In a recent study, Hammond et al. (8) identified a set of genes whose knockout resulted in female-specific sterility in Anopheles. However, they found that only one of these genes could be used as a target for HCGDs to achieve an efficient drive of female-specific sterility into the population. The remaining sterility genes imposed a very strong cost on the carriers that eventually resulted in the elimination of the drive allele from the population. As predicted by mathematical population genetics models, the spread of female-specific sterility traits in a population using HCGDs should eventually result in a population collapse and local or global elimination of the target species $(1,8)$. Another proposed strategy to achieve this goal is to design drive elements that alter the population's sex ratio toward males. Surprisingly, such gene-drive elements have naturally been observed in some organisms. In Aedes aegypti, for example, a type of drive element known as a "Killer-Y chromosome" is able to shatter the $\mathrm{X}$ chromosome during spermatogenesis, and therefore all offspring of mosquitoes carrying such a chromosome will be male. To replicate this phenomenon, Galizi et al. (9) employed a specific homing endonuclease, I-Ppol, to specifically shatter the $\mathrm{X}$ chromosome during spermatogenesis of Anopheles gambiae. By generating transgenic males carrying an engineered version of such a homing endonuclease gene (HEG) on somatic chromosomes, they have shown that at high initial load frequencies these flies will result in population 
collapse in cage experiments. They proposed that integration of such a HEG on the $\mathrm{Y}$ chromosome could be an effective gene-drive strategy for population control of An. gambiae. The distortion of the sex ratio using an X chromosome-specific CRISPR/Cas9 system has also been shown to be successful in An. gambiae(10).

Here, we propose an independent approach that converts female individuals into fertile males by disturbing the developmental sex-determination pathways, which distorts the sex ratio without adverse effects on the reproductive success of carrier males. A prime target gene to achieve this goal is transformer (tra). Tra plays a pivotal role in female sex determination in different insect orders, including Diptera (11). In a devastating agricultural fruit pest, the Mediterranean fruit fly, Ceratitis capitata ("medfly"), tra-knockdown XY males develop normally, while XX individuals develop as fertile males (12). Therefore, C. capitata XX males carrying a CHE-targeted tra locus could further spread the CHE to all their progeny (Fig. $1 \mathrm{~A})$, resulting in an effective gene drive without any direct effect on the fecundity of individuals carrying the drive element. This in theory could lead to an all-male population collapse that can be used for controlling the wild population of this aggressive pest.

Because of the strict guidelines on gene-drive experiments and to adhere to recommendations of scientific communities (13-15), we decided to test this gene-drive strategy first using Drosophila melanogaster as a model organism. In D. melanogaster, tra-mutant XX individuals develop into infertile pseudomales (16), not giving rise to further progeny (Fig. 1B). Since the cost of this infertility is significantly higher than the threshold tolerated by gene-drive systems $(1,4)$, a CHE targeting the tra locus in $D$. melanogaster, despite its ability to show superMendelian inheritance in individual crosses, is not able to drive into a population (Fig. 1C and D). This biological confinement allows us to employ D. melanogaster as a safe model organism for studying the limitations of our suggested suppression gene-drive systems at the molecular level in the laboratory and thereby experimentally identify parameters that might need to be adjusted to achieve an efficient suppression gene-drive system in C. capitata.

In our study, we found that targeting tra works as an efficient means of sex conversion in $D$. melanogaster. However, the early onset of the formation of in-frame drive-resistant alleles com-promises drive efficiency. Based on our observations, we simulated the use of a tra-based suppression gene-drive system for control of $C$. capitata populations and showed that HCGD systems employing multiple gRNAs that target the tra locus can serve as an effective pestcontrol strategy for C. capitata.

\section{Results}

\section{Design of a tra-Based Sex Conversion-Suppression Gene-Drive System.}

The proposed CHE is composed of an spCas 9 -coding sequence under the control of a suitable promoter, as explained below, a gRNA targeting the first exon of tra under the control of a 
PolIII promoter, and a fluorescent marker to identify the genomic integration (SI Appendix, Fig. S1C). The activity of this CHE unit will be similar to that of homing endonucleases and would be able to perform homing into the wild-type tra allele. For our tra-targeting CHE to drive in a population, it is essential that Cas9 is expressed in the germ cells to promote homing into the wild-type tra allele by HDR. To achieve sex conversion, however, tra needs to be inactivated in the somatic cells of XX individuals. Thus, two scenarios in XX individuals heterozygous for the drive allele are plausible: (i) Cas9 protein is expressed only in a fraction of the cells, and its activity results in the development of mosaic intersex individuals or (ii) Cas9 is expressed in all somatic cells and uniformly destroys the wild-type tra allele, resulting in the development of XX males. In C. capitata, the latter will result in development of fertile XX males (12), which can further spread the drive allele into the population (Fig. 1A). It is important to note that it is irrelevant whether the mutation of the wild-type tra allele in the somatic cells is based on HDR or NHEJ as long as the mutation disrupts the function of tra and thereby causes sex conversion.

Therefore, the combination of germline homing at the tra locus (which results in the spread of the drive allele) and somatic targeting of the wild-type tra allele (which results in sex conversion) is needed to enable our proposed suppression gene-drive strategy to be effective. To achieve this, different types of promoters or combinations thereof could be used. (i) A germline-specific pro-moter could be combined with an early zygotic promoter from a cellularization gene for high and ubiquitous blastoderm expression (17). Such cellularization promoters have already been success-fully applied for transgenic approaches in C. capitata (18). It is important to note that these early cellularization genes are not expressed in the primordial germ cell (PGC) nuclei $(19,20)$, which are therefore not exposed to NHEJ-based mutation in the early embryo $(21,22)$. In $D$. melanogaster, one of these cellularization genes, Sry- $\alpha$, is in fact expressed both in a somatically limited way in the blastoderm and in the PGCs at later developmental stages $(23,24)$, and its promoter therefore might be sufficient for both germline homing and somatic sex conversion. (ii) Since PolII-dependent transcription is actively suppressed in the PGCs (25), a ubiquitous cell cycle-specific promoter, such as the DNApol- 180 promoter (26), could result in uniform targeting of all cells during development except early-stage PGCs. The paternal-only transmission of our proposed genedrive strategy is likely to help overcome the problem of DNA cleavage at early embryonic stages when HDR is unlikely to occur $(21,22)$ and therefore is expected to result in both uniform sex conversion and germline homing. (iii) Since the target gene tra is expressed in the somatic cells at very early embryonic stages, the genomic context might mediate suitable amounts of expression independently of the introduced promoter. Thus, the introduction of a germline-specific promoter, such as the $R c d-1 r$ promoter, which had previously been shown to result in efficient homing-based gene drive in $D$. melanogaster (27), might by itself be sufficient to drive Cas9 expression for both purposes. 


\section{D. melanogaster as a Safe Model System for Evaluation of a tra-Based Suppression} Gene Drive.

In our experiments, we followed the recommended physical containment procedures (15) (SI Ap-pendix, SI Materials and Methods). Moreover, since in D. melanogaster XX males are always sterile, the somatic sex conversion imposes a strong fitness cost on the XX individuals carrying the drive allele, which impedes the spread of the drive allele in the population $(1,4)$, rendering $D$. melanogastera safe model system to study this suppression gene-drive strategy at the molecular level (Fig. 1B). Nevertheless, to ensure that the use of a CHE against the tra locus in D. melanogaster is indeed biologically confined in case of an unlikely accidental escape, a deterministic model for an ideal scenario (homing efficiency of $90 \%$ and assuming that one-third of NHEJ events result in the formation of in-frame indels) based on predicted phenotypic outcomes of the drive in $D$. melanogaster was used. The modeling graphs demonstrate that, because of its high fitness cost, even at $90 \%$ initial frequency a CHE targeting the tra locus not only is unable to drive into a population but also is actively eliminated from the population (Fig. 1C). In this example, the presence of the drive allele at high frequencies may result in the generation of cleavage-resistant alleles, which theoretically could alter the genetic makeup of the population at the targeted locus (28). However, our results indicate that at the low release frequencies $(<1 \%)$ that are expected in case of an accidental release, the drive allele becomes eliminated from the population at very early stages without any significant effect on the wild population (Fig. 1D). Therefore, it is safe to assume that such a drive system is biologically confined in D. melanogaster and thus meets the recommendations for genedrive experiments $(13-15,28)$.

\section{Implementation of the tra-Based Suppression Gene-Drive System in $D$ melanogaster.}

Since our sex conversion-based gene-drive system requires both somatic and germline Cas 9 activity, we tested three different promoters (SI Appendix, Fig. S1): (i) the Sry- $\alpha$ promoter,

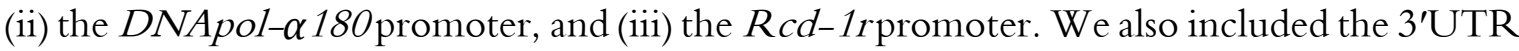
of the $\beta 2$ Tubulin ( $\beta$ Tub85D) gene at the $3^{\prime}$ end of the Cas9 transcript, as it had been shown to increase the homing efficiency in $D$. melanogaster (27). Moreover, the first intron of $\alpha T u b 84 B$ was inserted upstream of the Cas 9 coding sequence to further enhance Cas 9 expression (29).

To allow the simple generation of various strains in an isogenic background for these promoters, we used a transgenesis approach similar to that demonstrated in Anopheles (SI Appendix, Fig. S2) (8). First, a $t r a^{\mathrm{nDOCK}}$ strain was established by site-specific integration of a recombinase-mediated cassette exchange (RMCE) docking site into the first exon of the tra gene using an efficient $g R N A$ (SI Appendix, Fig. S1 A and B). Second, togenerate the homing strains for each of the promoters, RMCE was performed in tra ${ }^{\mathrm{nDOCK}}$ embryos using $\varphi \mathrm{C} 31$ 
integrase. All individuals that carried the CHE allele $\left(\operatorname{tra} a^{n C H E}\right)$ were found either to be males or to show a mosaic intersex phenotype, indicating that targeting the tra locus is indeed an efficient sex-conversion strategy in D. melanogaster (SI Appendix, Fig. S3A).

To assess the efficiency of each promoter in performing gene drive as well as inducing somatic sex conversion, 10 males from each of the $\operatorname{tra}^{n C H E}$ strains and the $\operatorname{tra}^{\mathrm{nDOCK}}$ strain were individually crossed with $w$-virgins, and the ratio of females in the F1 generation from each single cross was determined (SI Ap-pendix, Fig. S1D). The results show that all three promoters can block female development of heterozygous $\left(\operatorname{tra}^{n C H E /+}\right) \mathrm{XX}$ in-dividuals (somatic sex conversion) and drive into the next generation (germline activity). However, since HDR in the germline is of key importance for the molecular study of gene drive, we continued our experiments with the Rcd1rstrain.

To evaluate the drive efficiency of this CHE and the rate at which the tra locus is targeted to cause sex conversion, 12 heterozygous ( $\operatorname{tra}^{n C H E / \digamma}$ ) males were crossed individually with virgin $W^{-}$-flies. Screening the F1 progeny revealed that up to $92 \%$ of theindividuals carried the DsRed eye marker (on average $78 \%$, corresponding to a homing efficiency of $56 \%$ ), and up to $96 \%$ (on average 89\%) were males/intersexes (SI Appendix, Fig. S3B and C). These results further confirmed that our proposed suppression gene-drive strategy is indeed able to perform superMendelian inheritance, similar to findings in another recent study in D. melanogaster(21).

\section{Evolution of Cleavage Drive-Resistant tra Alleles.}

While we found our system to be highly efficient for sex conversion in D. melanogaster, we noticed during routine screening of the stocks the appearance of female flies with the DsRed eye marker phenotype. This was contrary to our previous observation and expectations that all heterozygous $\left(\operatorname{tra}^{n C H E /+)} \mathrm{XX}\right.$ flies should develop at least an intersex phenotype. Two scenarios could explain the presence of females with the DsRed eye marker: (i) an aborted or imperfect HDR, during which the DsRed eye marker is copied faithfully while an essential part of the drive element was lost or mutated, which would result in a dead CHE allele $\left(\operatorname{tra}^{n D}\right)$, or (ii) the presence of an in-frame mutation in the tra allele, which abolishes the recognition site of the gRNA without affecting the function of the tra-encoded protein $\left(\operatorname{tra}^{R s t}\right)$. Such mutations are likely to emerge from in-frame indel mutations as a result of NHEJ events induced by the CHE itself.

To check these hypotheses, virgin females with the DsRed eye marker were isolated and individually crossed with ${ }^{-}$-males. One of these crosses did not show any signs of an active drive system, with about $50 \%$ of the offspring showing the DsRed eyemarker. Molecular analysis of the mother and some female offspring from this cross revealed a large deletion in the CHE as the result of an aborted HDR event (SI Appendix, Fig. S4A). The other crosses, however, showed an efficient super-Mendelian inheritance, indicating the presence of an 
active CHE in the mother, which could be a sign of the presence of a tra ${ }^{R s t}$ allele in the mother. Sequencing the tra locus of these mothers confirmed the presence of in-frame indel mutations in the recognition site of the gRNA (similar to sequences in SI Appendix, Fig. $\mathrm{S} 4 \mathrm{~B})$. By crossing such females carrying an active CHE with $\operatorname{tra}^{n C H E}$ males, we were able to obtain homozygous $\operatorname{tra}^{n C H E} / \operatorname{tra}^{n C H E}$ males. When these homozygous males were crossed with $W$-virgins, all offspring were either male or intersex (SI Appendix, Fig.S3A), which further confirmed the high sex-conversion efficiency of this tra-targeting CHE.

To further evaluate the drive-resistant allele hypothesis and to estimate the rate at which resistant alleles may emerge from NHEJ events, we crossed heterozygous virgins carrying the

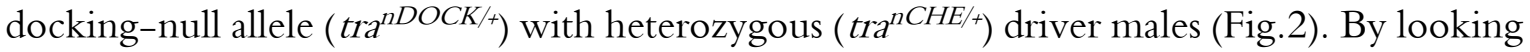
at the progeny that carry the $\operatorname{tra}^{n D O C K}$ allele (marked by ECFP fluorescence) but lack a $\operatorname{tra}^{n C H E}$ allele (DsRed fluorescence), we confined our analysis to situations of non-HDR at the paternal wildtype tra allele. Sequencing the tra allele in non-DsRed, ECFP females of the first generation resulted in the discovery of various independent in-frame indel mutations (SI Appendix, Fig.S5A). This suggests that drive-resistant alleles, $\operatorname{tra}^{R s t}$, are readily created as a result of NHEJ in heterozygous males that carry the CHE allele. To determine the frequency at which these $t r a^{R s t}$ alleles are generated, we crossed four heterozygous ( $\operatorname{tra}^{n C H E / \uparrow}$ ) males individually to $\operatorname{tra}^{n D O C K /}$ virgins and sequenced all progeny that showed only an ECFP fluorescence. We identified in-frame indels $\left(t r a^{R s t}\right)$ in up to $10 \%$ of all progeny, representing about one-third of all NHEJ events. The relative high emergence rate of such tra ${ }^{R s t}$ alleles in the F1 progeny demonstrates the rapid evolution of resistance as a direct consequence of an active homing CHE (SI Appendix, Fig.S5B) and confirms similar results from other groups $(21,30)$.

\section{Resistant Allele Dynamics and Spread.}

To estimate the dynamics of resistance allele emergence and spread in a population, we crossed (in five replicates each) $\mathrm{w}$-virgins with four different ratios of heterozygous $\operatorname{tra}^{n C H E /+}$ males to $W^{-}$males and followed the progeny for up to 15 generations. Thereby we documented the sex ratios as well as the spread of the DsRed-marked $\operatorname{tra}^{n C H E}$ allele, whose presence in females indicates potential drive-resistant $t r a^{R s t}$ alleles (Fig. 3 and SI Appendix, Figs.S6-S8). The ratio of such DsRed-positive females increased progressively over the generations, corresponding to the expected selective increase of resistance allele frequency. To characterize the molecular basis of the resistance to HCGD, we sequenced the tra locus from DsRed-fluorescent females from all the experimental settings at generation F6 and observed a diverse set of in-frame mutations representing drive-resistant $t r a^{R s t}$ alleles (SI Appendix, Fig. S4B). We also selected one setting for a molecular time-course analysis (setting D, replicate 4, at generations F1, F2, F6, and F13) and found that such mutations were heritable (SI Ap-pendix, Fig.S5C). The diversity of these in-frame indels across experimental settings and generations shows that these 
$\operatorname{tra}^{R s t}$ alleles are constantly created, independently of each other, at the site of cleavage. Interestingly, we already had observed DsRed-fluorescent females at the F1 generation of this replicate. These possessed a wild-type tra allele (SI Appendix, Fig.S5C) but contained a large deletion in the Cas9 gene of the $\operatorname{tra}^{n C H E}$ allele, which was likely the result of a rare, aborted HDR event (similar to that in SI Appendix, Fig.S4A). Following the populations to generation F15, we found an almost regular 1:1 ratio of males to females in all the replicates (Fig. 3 and SI Appendix, Fig.S8), independent of the original frequency of $\operatorname{tra}^{n C H E}$ allele inoculation (settings B and D).

\section{A tra-Based Suppression Gene-Drive System for C. capitata.}

Having shown the capability of our proposed CHE in inducing sex conversion in $D$. melanogaster and after identifying potential weaknesses of the system due to resistance evolution, we simulated the outcome of using our proposed method as a pest-control strategy in C. capitata (Fig. 4). Our population dynamics simulation results indicate that the evolution of in-frame drive-resistant alleles at rates that we observed in D. melanogaster would indeed impede a population collapse in C. capitata (Fig.4A), even if multiple releases were implemented in quick succession (Fig. 4C). To tackle the issue of in-frame drive-resistant alleles (these are problematic because they prevent homing while still allowing tra expression and hence are not removed due to a selective advantage), we considered the use of multiple $g R N A s$ to target the tra gene to reduce the proportion of resistant alleles that are in-frame. Using multiple gRNAs may not have a drastic effect on the overall NHEJ rate but will reduce the in-frame resistant allele formation rate exponentially with each additional gRNA, as each new target site would have to obtain an in-frame mutation that does not affect the function of the protein $(22,31)$. Our simulation study predicts that by using multiple $g R N A s$, and thereby reducing the generation rate of in-frame resistance alleles by at least two orders of magnitude, the effectiveness of the system is greatly improved. A single release is still not sufficient to achieve a population collapse in C. capitata (Fig. 4B); however, three releases in quick succession are sufficient (Fig. 4D).

The above-mentioned simulations are for the scenario in which $\mathrm{tra}^{n \mathrm{CHE} / *}$; XX individuals are infertile intersexes; however, we also explored the case in which these individuals are fertile males (Fig. 4E and F). In D. melanogaster, we observed that heterozygous ( $\operatorname{tra}^{n C H E / \uparrow}$ ) XX individuals develop into mosaic intersex individuals (SI Appendix, Fig.S3A). This is likely because the ectopic expression of Cas 9 under the control of the Rcd-1r promoter in only a proportion of the cells results in a mosaic phenotype. Since the intersex-based infertility of $\operatorname{tra}^{n C H E} \mathrm{XX}$ individuals places a fitness load on the system and reduces the drive (by preventing its occurrence in XX individuals), we propose the use of an early embryonic promoter, such as $S_{r y-\alpha}$, for the expression of Cas9.Expression from Sry- $\alpha$ in germ cells will allow gene drive to occur, and the early blastoderm expression guarantees a uniform destruction of the wild- 
type tra allele in all cells of the embryo at avery early stage; therefore heterozygous ( $\operatorname{tra}^{n C H E / \uparrow}$ ) XX individuals could develop into fertile medfly males. This in turn reduces the fitness load associated with the drive allele and increases the drive (by allowing super-Mendelian inheritance of the drive allele to occur in $\mathrm{tra}^{n \mathrm{CHE} /}$; $\mathrm{XX}$ individuals). Our simulation shows that enabling the fertility of heterozygous $\left(\operatorname{tra}^{n C H E / \uparrow}\right) \mathrm{XX}$ individuals does enhance the effectiveness of the system in collapsing a $C$. capitata population following a single release, provided that the in-frame drive-resistant allele generation rate is reduced by using multiple gRNAs (Fig. 4E and F). The tolerable generation rate of in-frame resistant alleles depends on the size of the targeted medfly population. Our simulations predict the extent by which this rate must be reduced to achieve a population collapse as a function of population size (SI Appendix, Fig.S9).

\section{Discussion}

Our mathematical modeling has shown that two main factors - the formation rate of the inframe resistance allele and the fitness of heterozygous ( $\mathrm{tra}^{n \mathrm{CHE} /+}$ ) sex-converted $\mathrm{XX}$ individuals - can havea significant effect on the expected outcome of a release in the wild. When heterozygous XX individuals are infertile, our model predicts that a population collapse can be achieved only if multiple inundative releases of the driver males are performed (Fig. 4D). While, this limitation could potentially be overcome by using a nearly embryonic stage promoter such as $\operatorname{Sry}-\alpha$ (Fig. 4F), this may not be desirable to ensure the local treatment of an insect pest population without the potential concern about the elimination of an entire species. Similar considerations have also been brought forward by Prowse et al. (32) with respect to fighting invasive vertebrate species.

In addition, we have shown that in our system it is the generation rate of in-frame driveresistant alleles, rather than the overall NHEJ rate, that has a significant impact on the outcomeof release scenarios. This is of significant importance for species such as $D$. melanogaster (and possibly for related pest species such as Drosophila suzuki) that might seem semirefractory toward homing-based gene-drive strategies (27), as it indicates that future designs may not necessarily require an extremely high homing rate but that only lowering the formation rate of the in-frame resistance allele and thus employing multiple $g R N A s(22,31)$ might be sufficient for an efficient suppression gene-drivestrategy in such species.

Our results support the idea that using a CHE to target genes that are essential for femalespecific development in insects, such as tra, can effectively result in a gender-biased population, finally resulting in a population collapse. This provides a basis for further development of similar suppression gene-drive strategies to introduce a gender bias in wild populations of insect pest ssuch as the medfly or disease vectors. If such a gender bias can be sustained long enough, species-specific elimination of the target species can be achieved. If the HCGDs explored here were applied to efficient pest-control management, the strategy in 
which $\operatorname{tra}^{n C H E /+}$; XX individuals are infertile intersexes is safer, because it requires multiple releases to achieve population collapse and hence will cause a population collapse only where these releases are carried out.Overall, we provide here an example, an implementation strategy, and the mathematical modeling required for the design and optimization of a homing-based sex conversion-suppression gene-drive approach for local or global speciesspecific elimination of insect pest or disease vector species. Moreover, we show that only lowering the formation rate of in-frame drive-resistant alleles by employing multiple $g R N A s$ may be sufficient to achieve an effective suppression gene-drive outcome, which has important implications for the design of such systems in species that exhibit a low homing rate in their germ cells.

\section{Materials and Methods}

Detailed methods on cloning, transgenesis, screening, molecular analysis, stock keeping of $D$. melanogaster strains, population modeling, and simulations can be found in SI Appendix, SI Materials and Methods. 


\section{References}

1. Esvelt KM, Smidler AL, Catteruccia F, Church GM (2014) Concerning RNA-guided gene drives for the alteration of wild populations. eLife3: e03401.

2. Unckless RL, Clark AG, Messer PW (2017) Evolution of resistance against CRISPR/Cas9 gene drive. Genetics 205:827-841.

3. Champer J, Buchman A, Akbari OS (2016) Cheating evolution: Engineering gene drives to manipulate the fate of wild populations. Nat Rev Genet17:146-159.

4. Deredec A, Burt A, Godfray HCJ (2008) The population genetics of using homing endonuclease genes in vector and pest management. Genetics179:2013-2026.

5. Unckless RL, Messer PW, Connallon T, Clark AG (2015) Modeling the manipulation of natural populations by the mutagenic chain reaction. Genetics201:425-431.

6. Gantz VM, Bier E (2015) Genome editing. The mutagenic chain reaction: A method forconverting heterozygous to homozygous mutations. Science348:442-444.

7. Gantz VM, et al. (2015) Highly efficient Cas9-mediated gene drive for population modification of the malaria vector mosquito Anopheles stephensi. Proc Natl Acad Sci USA112:E6736-E6743.

8. Hammond A, et al. (2016) A CRISPR-Cas9 gene drive system targeting female reproduction in the malaria mosquito vector Anopheles gambiae. Nat Biotechnol 34:78-83.

9. Galizi R, et al. (2014) A synthetic sex ratio distortion system for the control of the human malaria mosquito. Nat Commun 5:3977.

10. Galizi R, et al. (2016) A CRISPR-Cas9 sex-ratio distortion system for genetic control. Sci Rep 6:31139.

11. Geuverink E, Beukeboom LW (2014) Phylogenetic distribution and evolutionary dynamics of the sex determination genes doublesex and transformer in insects. Sex Dev 8:38-49.

12. Pane A, Salvemini M, Delli Bovi P, Polito C, Saccone G (2002) The transformer gene in Ceratitis capitata provides a genetic basis for selecting and remembering the sexual fate. Development 129:3715-3725.

13. Oye KA, et al. (2014) Regulating gene drives. Science345:626-628.

14. Lunshof J (2015) Regulate gene editing in wild animals. Nature 521:127

15. Akbari OS, et al. (2015) Safeguarding gene drive experiments in the laboratory. Science 349:927-929

16. Brown EH, King RC (1961) Studies on the expression of the transformer gene of Drosophila melanogaster. Genetics 46:143-156.

17. Horn C, Wimmer EA (2003) A transgene-based, embryo-specific lethality system for insect pest management. Nat Biotechnol 21:64-70. 
18. Schetelig MF, Caceres C, Zacharopoulou A, Franz G, Wimmer EA (2009) Conditional embryonic lethality to improve the sterile insect technique in Ceratitis capitata (Diptera: Tephritidae). BMC Biol 7:4.

19. Martinho RG, Kunwar PS, Casanova J, Lehmann R (2004) A noncoding RNA is required for the repression of RNApolII-dependent transcription in primordial germ cells. Curr Biol 14:159-165.

20. Lécuyer E, et al. (2007) Global analysis of mRNA localization reveals a prominent role in organizing cellular architecture and function. Cell 131:174-187.

21. Champer J, et al. (2017) Novel CRISPR/Cas9 gene drive constructs reveal insights into mechanisms of resistance allele formation and drive efficiency in genetically diverse populations. PLoS Genet13: e1006796.

22. Champer J, et al. (2018) Reducing resistance allele formation in CRISPR gene drives. Proc Natl Acad Sci USA115:5522-5527.

23. Schweisguth F, Yanicostas C, Payre F, Lepesant J-A, Vincent A (1989) cis-regulatoryelements of the Drosophila blastoderm-specific serendipity $\alpha$ gene: Ectopic activation in the embryonic PNS promoted by the deletion of an upstream region. Dev Bio 1136:181-193.

24. Fisher B, et al. (2012) BDGP insitu homepage. Available at insitu.fruitfly.org/cgibin/ex/insitu.pl. Accessed April 19, 2018

25. Timinszky G, Bortfeld M, Ladurner AG (2008) Repression of RNA polymerase II transcription by a Drosophila oligopeptide. PLoS One 3: e2506.

26. Hirose F, Yamaguchi M, Handa H, Inomata Y, Matsukage A (1993) Novel 8-base pair sequence (Drosophila DNA replication-related element) and specific binding factor involved in the expression of Drosophila genes for DNA polymerase alpha and proliferating cell nuclear antigen. J Biol Chem 268:2092-2099.

27. Chan YS, Huen DS, Glauert R, Whiteway E, Russell S (2013) Optimising homing endonuclease gene drive performance in a semi-refractory species: The Drosophila melanogaster experience. PLoS One 8: e54130

28. Noble C, Adlam B, Church GM, Esvelt KM, Nowak MA (2017) Current CRISPR gene drive systems are likely to be highly invasive in wild populations. bioRxiv, 10.1101/219022.

29. Duncker BP, Davies PL, Walker VK (1997) Introns boost transgene expression in Drosophila melanogaster. Mol Gen Genet254:291-296.

30. Hammond AM, et al. (2017) The creation and selection of mutations resistant to agene drive over multiple generations in the malaria mosquito. PLoS Genet 13: e1007039

31. Noble C, Olejarz J, Esvelt K, Church G, Nowak M (2017) Evolutionary dynamics of CRISPR gene drives. Sci Adv 3: e1601964

32. Prowse TAA, et al. (2017) Dodging silver bullets: Good CRISPR gene-drive design is critical for eradicating exotic vertebrates. Proc Biol Sci2 84:20170799. 


\section{Figures}
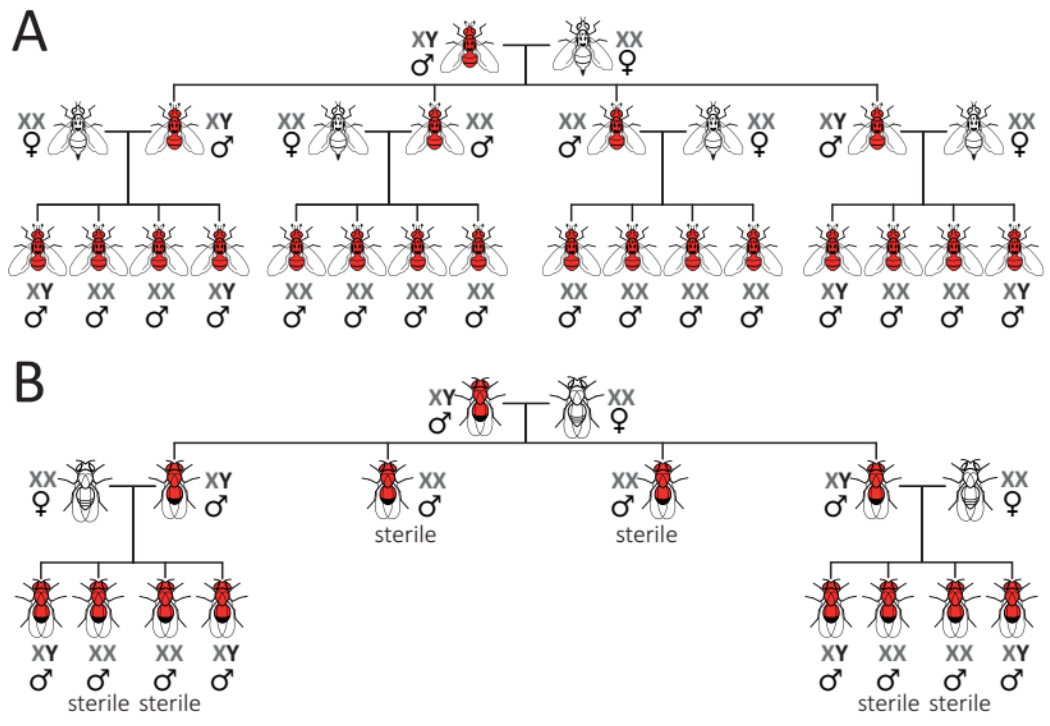

$\mathrm{C}$

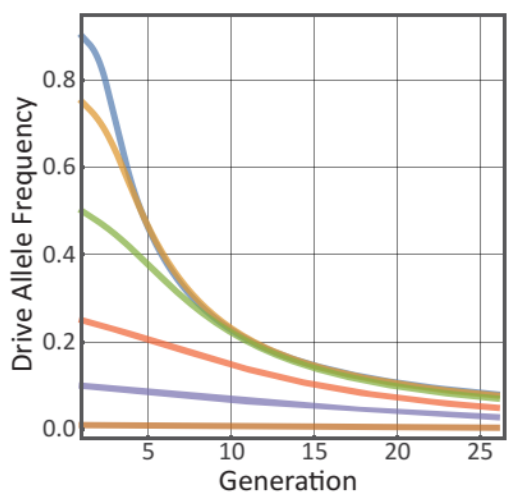

D

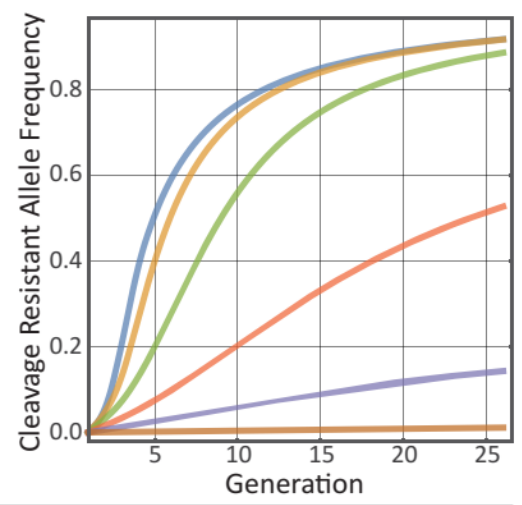

$$
\begin{array}{|l}
\text { Drive Allele Frequency at Release: } \\
\square 90 \% \quad \square 75 \% \quad \square 50 \% \quad \square 25 \% \quad \square 10 \% \quad \square 1 \%
\end{array}
$$

Fig. 1. Insect suppression gene drive based on forced all male offspring. (A) In C. capitata, a tra targeting Cas9 Homing Element (CHE) with both germline and somatic expression will cause super-Mendelian inheritance of the red fluorescence-marked CHE null allele, but also results in transformation of XX individuals into males and in theory lead to a subsequent collapse of the population. (B) In D. melanogaster, homing into tra, in somatic cells transforms XX individuals into sterile pseudo-males, which halts the spread of the selfish element, but allowed us to safely study the dynamics and molecular consequences of using CHEs in a suppression gene drive system. (C) Predicted transience of a Cas9-based homing construct targeting the tra locus in D. melanogaster. Predictions are based on an introduction of $\operatorname{tra}^{n C H E} /+$ males at frequencies of $1-90 \%$ into a population of otherwise wild type males and females in equal proportion. We assume a Cas9-mediated cleavage efficiency of $100 \%$, a probability of accurate homology-directed repair (HDR) following cleavage of $90 \%, \frac{1}{3}$ of drive-resistant alleles (NHEJ products) being in-frame indels, and with no fitness cost associated with in-frame drive-resistant alleles. A construct having these parameter values and released in the form of $\operatorname{tra}^{n C H E} /+; \mathrm{XY}$ males is expected to be eliminated from the population within $\sim 25$ generations regardless of the introduction frequency. (D) Although at high release frequencies, presence of the drive allele results in generation and establishment of cleavage resistant alleles in the population. At low release frequencies, which may occur because of accidental escapes, the drive allele will be eliminated early, and as a consequence the cleavage resistance alleles will only appear at negligible frequencies. This indicates that $D$. melanogaster is a safe model organism for the evaluation of a tra-based suppression gene drive causing sex conversion. 


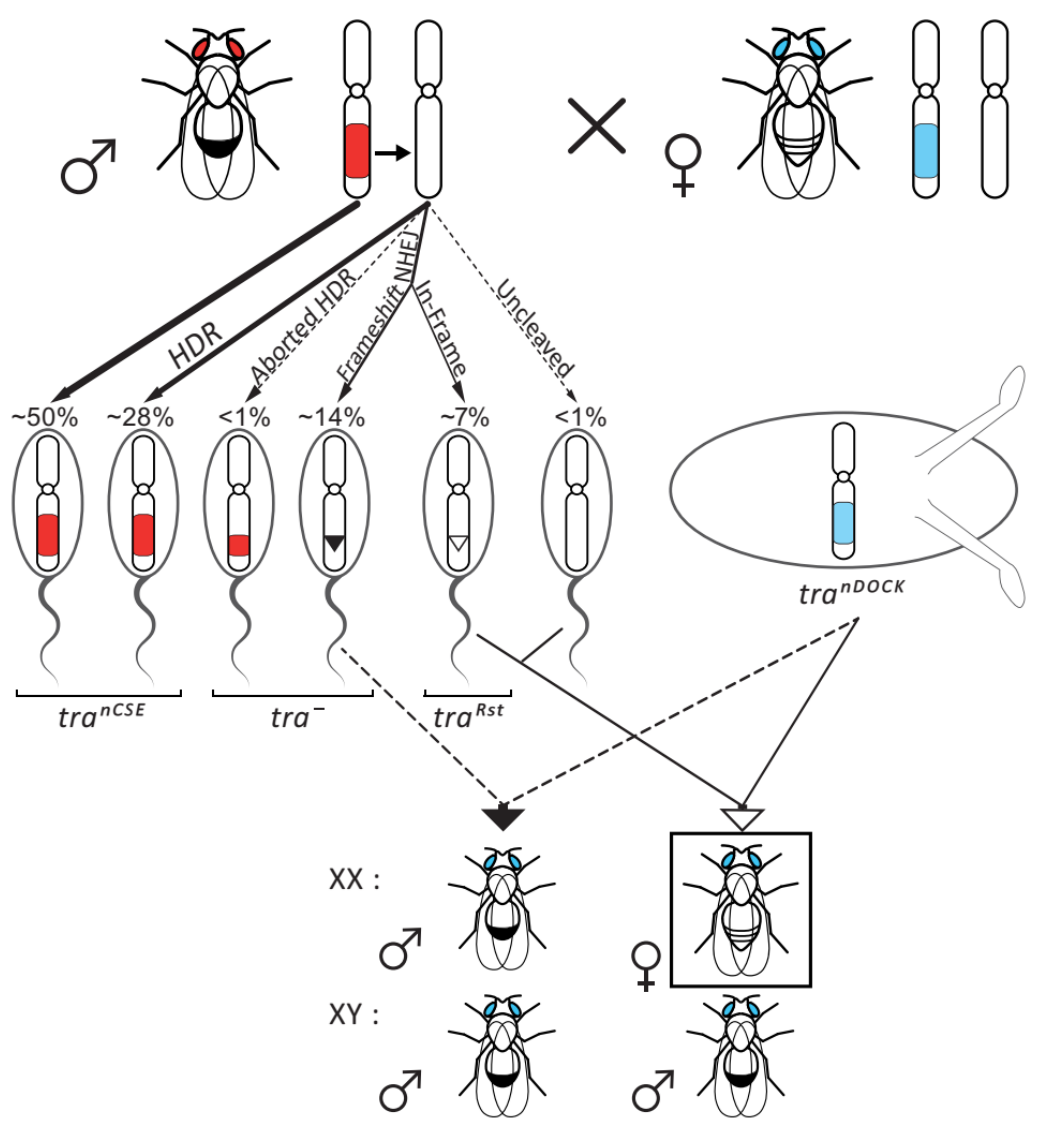

Fig. 2. CHE-targeting of the homologous gene locus. By analyzing the ECFP/non-DsRed progeny of $\operatorname{tra}^{n C H E} /+$ males and $\operatorname{tra}^{n D O C K} /+$ virgins, we focused on the non-HDR targeting events at a single tra locus. Values above each sperm indicate an estimation of each genotype based on the observed efficiency values. Molecular analysis of the CHE target site in $\mathrm{F}_{1}$ female progeny (boxed) identified independent NHEJ events causing various in-frame indels that resulted in drive-resistant functional alleles (SI Appendix, Fig. S5A). 

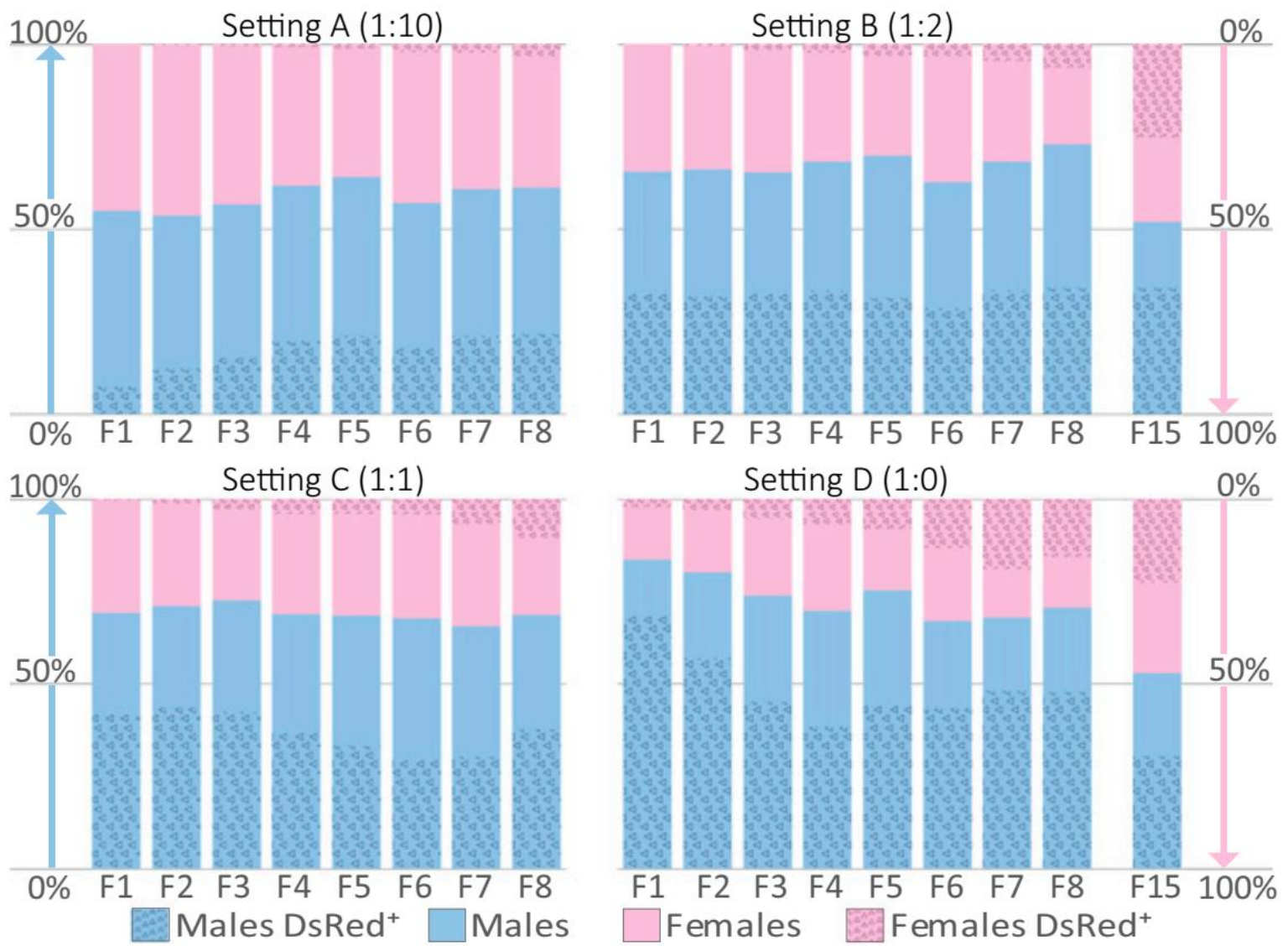

Fig. 3. Dynamics of sex ratio and indicated resistance allele spread in population experiments. $W$ virgins were crossed with various ratios of CHE ( $\left.\operatorname{tra}^{n C H E} /+\right)$ and wild-type $\left(W^{-}\right)$males (settings A-D). For each setting five replicates were carried out (SI Appendix, Fig. S6). Progenies were screened for sex and presence of the DsRed eye marker for up to 15 generations. In setting D, where only $\operatorname{tra}^{n C H E}$ males were used, a sex ratio of over $80 \%$ males was achieved within one generation, which indicates the collapse potential of this forced male-only offspring system. In XX embryos, $\operatorname{tra}^{n C H E}$ attacks the wild type tra locus resulting in inter-sex individuals (SI Appendix, Fig. S2). Thus, only females carrying non-functional defective $t r a^{n C H E}$ or drive-resistant functional $t r a^{R s t}$ alleles can show the DsRed marker. Therefore, the DsRed marker serves as an indicator for the $\operatorname{tra}^{R s t}$ allele presence in females and the rise in the percentage of DsRed females indicates the resistance spread in the population. Screening the $\mathrm{F}_{15}$ progenies in settings $\mathrm{B}$ and $\mathrm{D}$ showed that the populations adopted to the presence of the $\operatorname{tra}^{n C H E}$ homing allele with the female sex ratio reaching back to about $50 \%$ (SI Appendix, Fig. S6). 
A

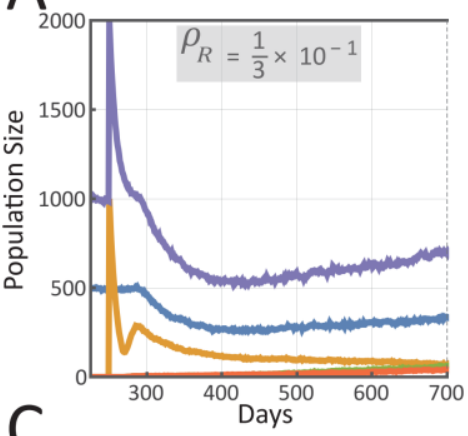

$\mathrm{c}$

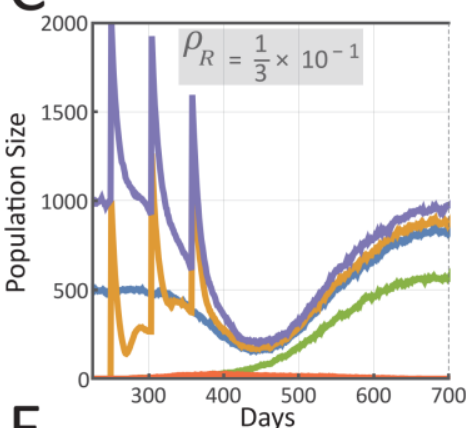

$\mathrm{E}$

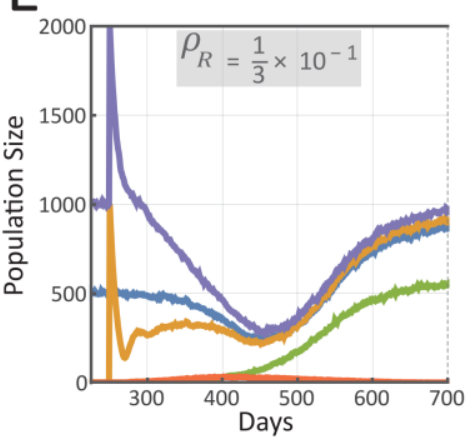

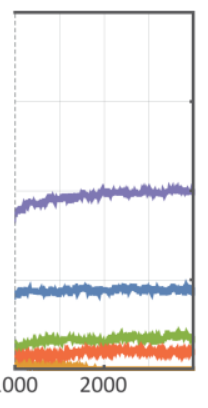
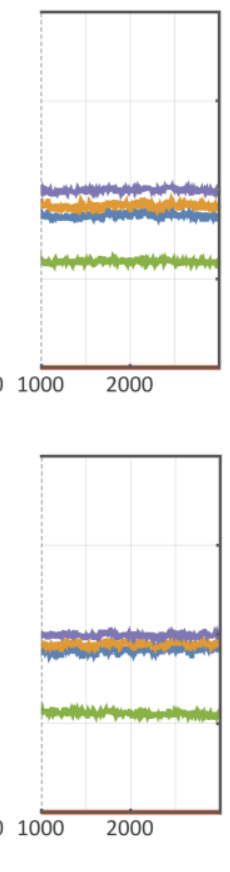

B

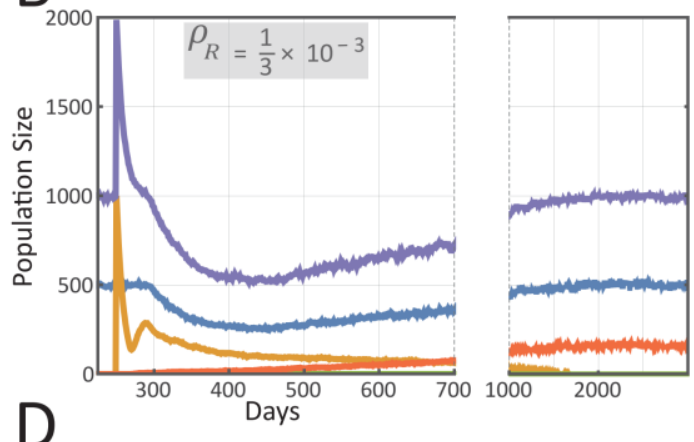

$\mathrm{D}$

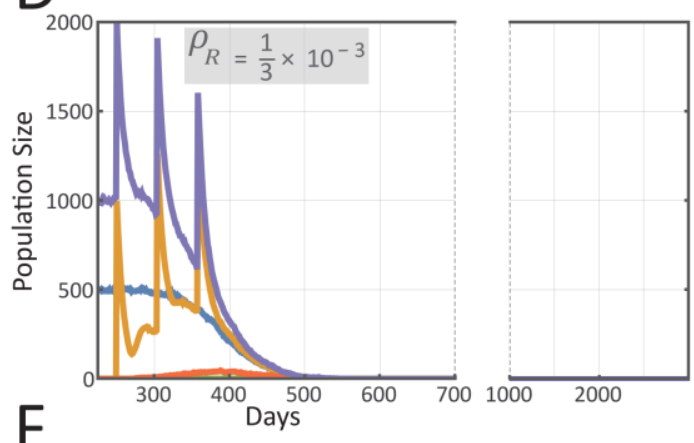

$\mathrm{F}$

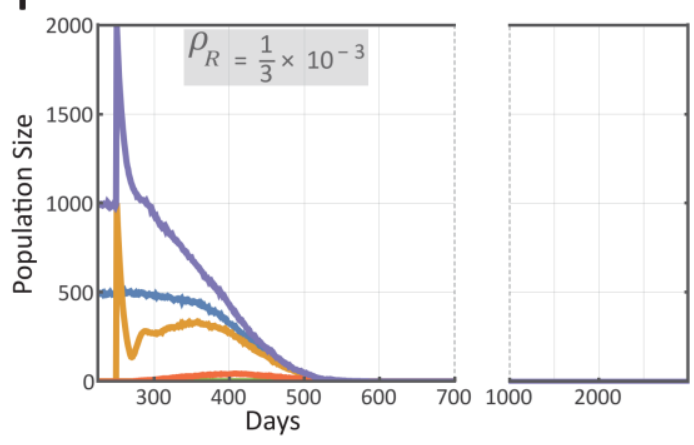

Total $\square$ Females $\square$ tra ${ }^{\text {nCSE }} \quad \square$ tra ${ }^{\text {Rst }} \quad \square$ tra $^{-}$

Fig. 4. Predicted dynamics of a Cas9-based homing system targeting the tra locus in $C$. capitata. Predictions are based on the population genetic model depicted in SI Appendix, Fig. S10 combined with the population dynamic model depicted in SI Appendix, Fig. S11 in which the life cycle of $C$. capitata is divided into four stages - egg, larva, pupa and adult - with density-dependent mortality occurring at the larval stage (SI Appendix, Table S1). Homing only occurs in $\operatorname{tra}^{\mathrm{nCHE}} /+$ heterozygotes, where + represents the wild type allele and $\operatorname{tra}^{n C H E}$ represents the intact drive allele. We assume a Cas9mediated cleavage efficiency of $100 \%$ and a probability of accurate homology-directed repair following cleavage of $90 \%$ (NHEJ rate $(\delta)=0.1$ ). By default, in-frame drive-resistant alleles $\left(\operatorname{tra}^{\mathrm{Rst}}\right)$, account for $\frac{1}{3}$ of generated resistant alleles, although this proportion may be reduced through gRNA multiplexing. The remaining cleavage resistant alleles, are out-of-frame or other mutations that result in a tra null allele. The equilibrium population size of $C$. capitata is 1,000. Releases consist of $1,000 \operatorname{tra}^{n C H E}$; XY males once or at intervals. In panels A-D, the scenario in which $\operatorname{tra}^{n C H E}$; XX individuals are infertile intersex is considered. (A) For a homing efficiency of $90 \%$ and an in-frame resistant allele generation rate $\left(\rho_{\mathrm{R}}=\delta \theta\right.$, where $\delta$ is the NHEJ rate and $\theta$ is the fraction of NHEJs that produce in-frame indels) of $\frac{1}{3}$ of $10 \%$, a single release of $1,000 \operatorname{tra}^{n C H E}$; XY males results in temporary population suppression, halving the adult population size, with the population rebounding over a period of several years. (B) Decreasing the in-frame drive-resistant allele generation rate, $\rho_{R}$, by two orders of magnitude to $\frac{1}{300}$ of $10 \%$, and hence increasing the out-of-frame resistant allele generation rate, $\rho_{\mathrm{B}}=\delta(1-\theta)$, to $\sim 10 \%$, the population suppression is still only moderate and transient. (C) If three releases of 1,000 tra ${ }^{n C H E}$; XY males are carried out in succession, the extent of population suppression is much greater (>75\% suppression); however, at a $\rho_{\mathrm{R}}$ of $\frac{1}{3}$ of $10 \%$, the population still rebounds over a period of several years with an increase in the frequency of $\operatorname{tra}^{R s t}$ alleles. (D) Nevertheless, if three consecutive releases are carried out for a construct with the decreased in-frame drive-resistant allele generation rate, population 
elimination can be achieved within $\sim 1$ year after the last release. (E) In panels E-F, the scenario in which $\operatorname{tra}^{n C H E}$; XX individuals are fertile males is considered. For a homing efficiency of $90 \%$ and an in-frame resistant allele generation rate, $\rho_{R}$, of $\frac{1}{3}$ of $10 \%$, a single release of $1,000 \mathrm{tra}^{n C H E}$; XY males results in temporary population suppression, as in-frame driveresistant alleles become prevalent, preventing population elimination. $(F)$ However, if the in-frame resistant allele generation rate, $\rho_{\mathrm{R}}$, is reduced by two orders of magnitude to $\frac{1}{300}$ of $10 \%$, the emergence of in-frame drive-resistant alleles is unlikely, and the population can be eliminated following a single release of 1,000 $\mathrm{tra}^{n C H E}$; XY males. 


\section{SUPPLEMENTARY INFORMATION (SI) APPENDIX:}

Consequences of instant induction of resistance evolution on a sex conversionbased suppression gene drive for insect pest management

Mohammad KaramiNejadRanjbar ${ }^{\mathrm{a}, 1,2}$, Kolja N. Eckermann ${ }^{\mathrm{a}, 1}$, Hassan M. M. Ahmed ${ }^{\mathrm{a}, 1}$, Héctor M. Sánchez C. ${ }^{\text {, }}$ Stefan Dippel ${ }^{\mathrm{a}}$, John M. Marshall ${ }^{\mathrm{b}}$, Ernst A. Wimmer ${ }^{\mathrm{a}, 3}$

${ }^{a}$ Department of Developmental Biology, Johann-Friedrich-Blumenbach-Institute of Zoology and Anthropology, Göttingen Center of Molecular Biosciences, University of Goettingen, 37077 Göttingen, Germany. ${ }^{b}$ Divisions of Biostatistics and Epidemiology, School of Public Health, University of California, Berkeley, CA 94720 USA

To whom correspondence should be addressed: Ernst A. Wimmer,E-mail: ewimmer@gwdg.de 


\section{Materials and Methods}

Computational modeling of gene drive in $\boldsymbol{D}$. melanogaster. Equation B4 from the model by Unkless et. al. for resistance evolution in gene drive (147) was expanded as below to include all the important parameters of our study, where $\mathrm{x}_{\mathrm{w}}, \mathrm{x}_{\mathrm{d}}, \mathrm{x}_{\mathrm{r}}$ and $\mathrm{x}_{\mathrm{b}}$ are allele frequencies for wild-type, driver, in-frame resistance, and frameshift alleles, superscripts represent the sex. Parameters $c, \delta$, and $\theta$ represent cleavage rate, NHEJ rate, and fraction of NHEJs that produce in-frame indels, respectively. $\omega_{\mathrm{ab}}$ shows the fitness of an individual with genotype $\mathrm{ab}$.

$$
\begin{gathered}
\bar{\omega}(t)=\left(x_{w}^{m} x_{d}^{f}+x_{d}^{m} x_{w}^{f}\right)\left[(1-c) \omega_{d w}+c(1-\delta) \omega_{d d}+c \delta(1-\theta) \omega_{d b}+c \delta \theta \omega_{d r}\right]+ \\
\left(x_{w}^{m} x_{r}^{f}+x_{r}^{m} x_{w}^{f}\right) \omega_{r w}+\left(x_{w}^{m} x_{b}^{f}+x_{b}^{m} x_{w}^{f}\right) \omega_{b w}+\left(x_{d}^{m} x_{r}^{f}+x_{r}^{m} x_{d}^{f}\right) \omega_{d r}+\left(x_{d}^{m} x_{b}^{f}+x_{b}^{m} x_{d}^{f}\right) \omega_{d b}+ \\
\left(x_{b}^{m} x_{r}^{f}+x_{r}^{m} x_{b}^{f}\right) \omega_{r b}+\sum_{i} x_{i}^{m} x_{i}^{f} \omega_{i i}
\end{gathered}
$$

For the specific case of $D$. melanogaster where $\omega_{\mathrm{dw}}, \omega_{\mathrm{dd}}, \omega_{\mathrm{db}}$, and $\omega_{\mathrm{bb}}$ are 0 for females and all other fitness values are considered 1, the following equations can be derived from equation E1 to recursively calculate the allele frequencies at each generation for females:

$$
\begin{gathered}
x_{d}^{\prime f}=\frac{\left(x_{w}^{m} x_{d}^{f}+x_{d}^{m} x_{w}^{f}\right) c \delta \theta+\left(x_{d}^{m} x_{r}^{f}+x_{r}^{m} x_{d}^{f}\right)}{2 \bar{\omega}_{D m}(t)} \\
x_{r}^{\prime f}=\frac{\left(x_{w}^{m} x_{d}^{f}+x_{d}^{m} x_{w}^{f}\right) c \delta \theta+\left(x_{w}^{m} x_{r}^{f}+x_{r}^{m} x_{w}^{f}\right)+\left(x_{d}^{m} x_{r}^{f}+x_{r}^{m} x_{d}^{f}\right)+\left(x_{b}^{m} x_{r}^{f}+x_{r}^{m} x_{b}^{f}\right)+2 x_{r}^{m} x_{r}^{f}}{2 \bar{\omega}_{D m}(t)} \\
x_{b}^{\prime f}=\frac{\left(x_{w}^{m} x_{b}^{f}+x_{b}^{m} x_{w}^{f}\right)+\left(x_{b}^{m} x_{r}^{f}+x_{r}^{m} x_{b}^{f}\right)}{2 \bar{\omega}_{D m}(t)} \\
x_{w}^{\prime f}=\frac{\left(x_{w}^{m} x_{r}^{f}+x_{r}^{m} x_{w}^{f}\right)+\left(x_{w}^{m} x_{b}^{f}+x_{b}^{m} x_{w}^{f}\right)+2 x_{w}^{m} x_{w}^{f}}{2 \bar{\omega}_{D m}(t)}
\end{gathered}
$$

and for males:

$$
\begin{array}{r}
x_{d}^{\prime m}=\frac{\left(x_{w}^{m} x_{d}^{f}+x_{d}^{m} x_{w}^{f}\right)[c(1-\delta)+1]+\left(x_{d}^{m} x_{r}^{f}+x_{r}^{m} x_{d}^{f}\right)+\left(x_{d}^{m} x_{b}^{f}+x_{b}^{m} x_{d}^{f}\right)+2 x_{d}^{m} x_{d}^{f}}{2 \bar{\omega}_{D m}(t)} \\
x_{r}^{\prime m}=\frac{\left(x_{w}^{m} x_{d}^{f}+x_{d}^{m} x_{w}^{f}\right) c \delta \theta+\left(x_{w}^{m} x_{r}^{f}+x_{r}^{m} x_{w}^{f}\right)+\left(x_{d}^{m} x_{r}^{f}+x_{r}^{m} x_{d}^{f}\right)+\left(x_{b}^{m} x_{r}^{f}+x_{r}^{m} x_{b}^{f}\right)+2 x_{r}^{m} x_{r}^{f}}{2 \bar{\omega}_{D m}(t)} \\
x_{b}^{\prime m}=\frac{\left(x_{w}^{m} x_{d}^{f}+x_{d}^{m} x_{w}^{f}\right)[c \delta(1-\theta)]+\left(x_{w}^{m} x_{b}^{f}+x_{b}^{m} x_{w}^{f}\right)+\left(x_{d}^{m} x_{b}^{f}+x_{b}^{m} x_{d}^{f}\right)+\left(x_{b}^{m} x_{r}^{f}+x_{r}^{m} x_{b}^{f}\right)+2 x_{b}^{m} x_{b}^{f}}{2 \bar{\omega}_{D m}(t)} \\
x_{w}^{\prime m}=\frac{\left(x_{w}^{m} x_{d}^{f}+x_{d}^{m} x_{w}^{f}\right)(1-c)+\left(x_{w}^{m} x_{r}^{f}+x_{r}^{m} x_{w}^{f}\right)+\left(x_{w}^{m} x_{b}^{f}+x_{b}^{m} x_{w}^{f}\right)+2 x_{w}^{m} x_{w}^{f}}{2 \bar{\omega}_{D m}(t)}
\end{array}
$$


where

$$
\begin{aligned}
& \bar{\omega}_{D m}(t)=\left(x_{w}^{m} x_{d}^{f}+x_{d}^{m} x_{w}^{f}\right)(1+c \delta \theta)+2\left(x_{w}^{m} x_{r}^{f}+x_{r}^{m} x_{w}^{f}+x_{w}^{m} x_{b}^{f}+x_{b}^{m} x_{w}^{f}+x_{d}^{m} x_{r}^{f}+x_{r}^{m} x_{d}^{f}+\right. \\
& \left.x_{b}^{m} x_{r}^{f}+x_{r}^{m} x_{b}^{f}+x_{w}^{m} x_{w}^{f}+x_{r}^{m} x_{r}^{f}\right)+x_{d}^{m} x_{b}^{f}+x_{b}^{m} x_{d}^{f}+x_{d}^{m} x_{d}^{f}+x_{b}^{m} x_{b}^{f}
\end{aligned}
$$

Guide RNA selection and HRMA. Several gRNAs targeting the first exon of the $D$. melanogaster tralocus were selected using CRISPR DESIGN online tool. Selected guide sequences (Fig. S1B) were cloned into the pCFD2 plasmid (148) (Addgene 49409, gift from S. Bullock) using annealed oligonucleotides for each gRNA (Fig. S1A). One hour old embryos from the act5-cas9 $D$. melanogaster strain (148) (Bloomington 54590) were injected using the construct for each gRNA. After 24 hours, individual embryos were homogenized in $50 \mu \mathrm{l}$ of smashing buffer $(10 \mathrm{mM}$ Tris- $\mathrm{HCl}$, pH 8.2, $25 \mathrm{mM} \mathrm{NaCl}, 1 \mathrm{mM}$ EDTA, $0.2 \%$ Triton x100 and $200 \mu \mathrm{g} / \mathrm{ml}$ Proteinase K) (149) using small tips. Samples were kept at $55^{\circ} \mathrm{C}$ for 1 hour followed by 5 minutes incubation at $95^{\circ} \mathrm{C}$ to heat inactivate Proteinase K. $1 \mu \mathrm{l}$ was used for PCR using MK078 and MK079 primers (Table S1) with EvaGreen qPCR master mix and High Resolution Melt curves (150) were obtained in a BioRad CFX96 realtime $\mathrm{C} 1000$ thermal cycler at $0.2^{\circ} \mathrm{C}$ steps. Relative fluorescent at $75^{\circ} \mathrm{C}$ and $85^{\circ} \mathrm{C}$ were normalized to 1 and 0 , respectively, and the control graph was subtracted from all the guide RNA graphs. The guide that showed the largest difference ( $\mathrm{g} 4$ ) was selected as the most efficient guide RNA. To make sure that off-target effects are minimal (150), three of the top similar sequence hits from the CRISPR DESIGN online tool (151) were checked for the most efficient guide (g4) using High Resolution Melting Analysis (HRMA) employing MK155/MK156, MK157/MK158 and MK159/MK160 primer pairs (Table S1).

Constructs. To generate the SG022 plasmid (pCRII-tra4R-attP-3xP3CFP-attP-tra4L, GeneBank KY171964), In-Fusion assembly (Clontech, USA) was performed on HindIII/ApaI digested pCRII (ThermoFisher, USA) backbone and PCR products of (i) MK024/MK122 primers on genomic DNA from the D. melanogaster strain Oregon-R for left homologous arm, (ii) MK117/MK116 primers on pBac\{3xP3-ECFPaf\} (72) for 3xP3-ECFP-SV40pA, and (iii) MK123/MK025 primers on genomic DNA from the Oregon-R strain for right homologous arm. MK116 and MK117 primers (Table S1) introduce attP sites (152) at both ends of the ECFP marker to generate a recombinase-mediated exchange cassette. The SG012 plasmid (pCFD3-g4) was generated by cloning annealed MK083 and MK044 oligonucleotides into the pCFD3 plasmid (148) (Addgene 49410, gift from S. Bullock) using the depositor's suggested protocol (153). To generate the SG011 plasmid (pCRII-attBSmaI), the pCRII vector was first digested with XbaI/HindIII followed by ligation of annealed MK060 and MK061 oligonucleotides (Table S1). Then the NsiI cut site in the vector was destroyed by first digesting the plasmid with NsiI followed by T4 DNA polymerase treatment and religation using T4 DNA Ligase. In-Fusion assembly was performed on the BamHI/NotI digested pIE4 plasmid (154) and PCR products of (i) MK072/MK075 primers (Table S1) on genomic DNA from Oregon-R strain for first intron of alpha-tub84B and (ii) MK076/MK077 primers on pBS-Hsp70-Cas9 (Addgene 46294, gift from M. Harrison, K. O'Connor-Giles, J. Wildonger) for D. melanogaster codon optimized SpCas9 coding sequence, to generate the SG020 plasmid (pIE4-aTubIGT1-Cas9). MK134 and MK135 primers (Table S1) were used to amplify the aTubI1-Cas9 fragment from SG020 plasmid. The 
fragment was directly ligated to SmaI digested SG011 to generate the SG023 plasmid (pCRII-attBaTubCas9-attB). For the SG024 plasmid (pCRII-attB-aTubCas9bTub-U63g4-DsRed-attB, GeneBank KY171962), in-Fusion assembly was performed on AscI digested SG023 plasmid and PCR products of (i) MK144/MK145 primers on genomic DNA from Oregon-R strain for the 3' UTR of beta-tub85D (155), (ii) MK147/MK146 primers on SG012 for U6:3-g4, and (iii) MK149/MK148 primers on $\mathrm{pBac}\{3 \times \mathrm{xP} 3-\mathrm{DsRedaf}\}$ (156) for 3xP3-DsRed-SV40pA. To amplify each of the promoters Oregon-R genomic DNA was used as template and (i) MK140/MK141 primer pairs (Table S1) were used to amplify a $900 \mathrm{bp}$ fragment of the Rcd-1r promoter (155), (ii) MK142/MK143 primer pairs (Table S1) were used to amplify a 550 bp fragment of the Sry- $\alpha$ promoter (157), and (iii) MK138/MK139 primer pairs (Table S1) were used to amplify a $500 \mathrm{bp}$ fragment of the DNApol- $\alpha 180$ promoter (158). The PCR products were then digested using XbaI (or AvrII) and XhoI restriction enzymes and the products were ligated to AvrII/XhoI digested SG024 plasmid to generate SG039 (pCRII-attB-Rcd1rp-aTubCas9bTub-U63g4-DsRed-attB， GeneBank KY171963)，ＳG040 (pCRII-attB-sryap-aTubCas9bTub-U63g4-DsRed-attB), and SG037 (pCRII-attB-DPol180paTubCas9bTub-U63g4-DsRed-attB) plasmids respectively. MK153 and MK154 primers (Table S1) were used to amplify the phiC31 coding sequence from the plasmid pcDNA3.1-phiC31 (159) (Addgene 68310, gift from K. Basler). The PCR product was then digested with BsaI and NotI restriction enzymes and was ligated to NcoI/NotI digested pSL[faHSfa] plasmid (160) to generate the SG042 helper plasmid (pSL-DmHsp70-phiC31-Hsp70).

Generation of the docking $\left(\boldsymbol{t r a}^{\mathrm{nDOCK}}\right)$ strain. To generate the docking strain (Fig. S2A), $\operatorname{tra}^{\mathrm{nDOCK}}$, 30 minutes old de-chorionated embryos from the act5-Cas9 D. melanogaster strain (148) were covered with hydrocarbon oil (Voltalef 10S) and injected with an injection mix containing $500 \mathrm{ng} / \mu \mathrm{l}$ of SG022 (pCRII-tra4R-attP-3xP3CFP-attP-tra4L) HDR donor plasmid and $300 \mathrm{ng} / \mu \mathrm{l}$ of SG012 (pCFD3-g4) gRNA-producing plasmid. Embryos were kept humid at $25^{\circ} \mathrm{C}$ for 24 hours on an apple agar plate. Newly hatched larvae were gently collected from the apple agar plate using a size 00 brush and placed on $D$. melanogaster food supplemented with dried yeast in a small vial. Larvae were kept at $25^{\circ} \mathrm{C}$ until eclosion and only male offspring were individually crossed with $W$ virgins. $\mathrm{F}_{1}$ third instar larvae from individual vials were collected from the food by applying $\mathrm{CO}_{2}$ to the media. The larvae were aligned on a cold microscope slide and screened under a Zeiss fluorescent binocular for the ECFP eye marker. Positive larvae from each cross were placed into new vials until eclosion. Individual male flies with the ECFP eye marker were then crossed with virgins from the $\left[\frac{w^{-}}{w^{-}} ; \frac{C y O(C y)}{S p} ; \frac{T M 3(S b)}{D r}\right]$ balancer strain. $F_{2}$ flies carrying the ECFP eye marker, $C y$ (Curly wings) and $S b$ (Stubble) phenotypes were self-crossed. $F_{3}$ flies carrying the ECFP eye marker and the $S b$ phenotype without any of the other balancer phenotypes were kept to establish the strain. Molecular analysis was performed to verify the proper genome editing at the third chromosomal tra locus that generated $\operatorname{tra} a^{\mathrm{nDOCK}}$.

Molecular Characterization of $\boldsymbol{t r a}^{n D O C K}$ strain. To confirm the correct integration of the docking cassette into the tra locus, MK126/MK128 and MK127/MK129 primer pairs (Table S1) were used to amplify products of $\sim 3 \mathrm{kbp}$ from the $\operatorname{tra}^{n D O C K}$ genomic DNA. Templates were prepared by homogenizing the head of individual flies in $50 \mu \mathrm{l}$ of smashing buffer (149) using small tips. MK126 and MK127 primer binds to regions upstream and downstream of the Left and Right Homologous Arms used for the HDR, respectively. MK128 and MK129 primers, however, bind to 3' end and 5' 
end of the ECFP CDS, respectively. Therefore, a product of around $3 \mathrm{kbp}$ from each of these primer pairs can only form if integration at the correct locus has occurred. Off-target integration does not result into an amplification product with these primer pairs because MK126 and MK127 primers bind outside the homologous arms regions. One of the fly strains that passed all of the quality control criteria was then kept as a stock.

Generation of the homing $\operatorname{tra}^{n C H E}$ strain. To generate the homing $\operatorname{tra}^{n C H E}$ strains (Fig. S2B), 30 minutes old embryos de-chorionated from the docking strain $\left(\operatorname{tra} a^{n D O C K}\right.$ ) were covered with hydrocarbon oil and injected with an injection mix containing $500 \mathrm{ng} / \mu \mathrm{l}$ of each of the donor plasmids for respective promoters (SG039, SG040, and SG037) and $300 \mathrm{ng} / \mu \mathrm{l}$ of SG042 (pSL-DmHsp70phiC31-Hsp70) helper plasmid to perform a Recombinase-Mediated Cassette Exchange (RMCE) (161). Embryos were kept humid at $25^{\circ} \mathrm{C}$ for 24 hours on an apple agar plate. Newly hatched larvae were gently collected from the apple agar plate using a size 00 brush and placed on $D$. melanogaster food supplemented with dried yeast in a small vial. Larvae were kept at $25^{\circ} \mathrm{C}$ until eclosion. Newly eclosed flies were individually crossed with $\bar{W}^{-}$flies. For each of the promoters $\mathrm{F}_{1}$ offspring was screened for the absence of ECFP and the presence of DsRed eye marker. Individual males with only the DsRed eye marker were then crossed with $W^{-}$virgins. Positive $\mathrm{F}_{2}$ individuals (consisting of males/intersexes only) from each single cross were then pooled together and $W^{-}$virgins were added to the pool. Few of the $\mathrm{F}_{2}$ males/intersexes were used for DNA extraction for molecular characterization and verification of RMCE. To sustain the strain over generations, a few $\bar{W}$ virgins were added to the vials every two weeks.

Molecular Characterization of Red-1r $\operatorname{tra}^{n C H E}$ strains. To confirm the fidelity of the RMCE and also to determine the orientation of the integration, MK073/MK078 and MK073/MK079 primer pairs (Table S1) were used to amplify a $\sim 2 \mathrm{kbp}$ region from the $\operatorname{tra}^{n C H E}$ genomic DNA. Templates were prepared by homogenizing the head of individual flies in $50 \mu \mathrm{l}$ of smashing buffer (149) using small tips. MK073 binds to the upstream of the SpCas9 coding sequence within the CHE. MK078 and MK079 bind to upstream and downstream of the g4 target site, respectively. A $2 \mathrm{kbp}$ amplification product with MK073/MK078 primer pairs indicates a sense integration event (endogenous tra and SpCas9 CDSs on the same strand). However, a $2 \mathrm{kbp}$ amplification product with MK073/MK079 primer pairs indicates an anti-sense integration event (endogenous tra and SpCas9 CDSs on opposite strands). One of the fly strains with an anti-sense integration that passed all the quality control criteria was kept as a stock and was used for all further experiments.

Stock keeping of $\operatorname{tra}^{n C H E}$ strains. Despite the fact that the high cost of drive in our $\operatorname{tra}^{\mathrm{nCHE}} D$. melanogasterstrains does not allow for spread of the drive allele in any population and thus serves as a very effective biological confinement strategy (Fig. $1 \mathrm{C}$ and D), we carried out stock keeping of this homing $\operatorname{tra}^{n C H E}$ strain as well as all experiments generating and using this strain with utmost care to not have potential gene drive individuals escape the laboratory. All experiments were performed in our well-equipped safety level one (S1) laboratory, but only in windowless internal rooms, with ventilation in- and outlets covered with tight mesh and doors supplied with brushes. Flies were always anesthetized before opening containers as well as analyzed and sorted under constant anesthetization. Fly traps were installed in the rooms and in the neighboring corridors. For transport between rooms, flies were put in double-walled containers. Before discarding, flies were finally deep frozen for more 
than twelve hours. Despite the design and commencement of our study in 2013, it already met the criteria for safeguarding gene drive experiments in the laboratory as published in Science in 2015 (162) since two stringent confinement strategies were employed: (i) biological confinement based on the high cost of this specific gene drive in $D$. melanogaster and (ii) organizational as well as physical containment based on the rules for handling this strain in our S1 laboratory. In 2016, we were informed of the new guidelines on handling gene drive experiments in Germany by the German Central Commission for Biological Safety (ZKBS) that suggested to perform such experiments only in safety level two (S2) laboratories. Therefore, we stopped our experiments and moved our $\operatorname{tra}^{n C H E}$ strain temporarily to a neighboring S2 laboratory for stock maintenance only. At the same time, we applied for a single case evaluation of our experiments with the ZKBS that approved of further handling the $\operatorname{tra}^{n C H E}$ strain in our S1 laboratory with the above-mentioned measures (file reference: ZKBS 45110.1933). After publication of this study, we will discard the strain that can easily be re-generated by RMCE from the docking line $\operatorname{tra} a^{\mathrm{nDOCK}}$.

Estimating the sex conversion and homing efficiencies. The sex conversion efficiency of the $\operatorname{tr} a^{\mathrm{nCHE}}$ allele was evaluated by crossing twelve homozygous $\operatorname{tra}^{\mathrm{nCHE}} / \operatorname{tra}^{\mathrm{nCHE}}$ males individually with three $\bar{W}$ virgins each. The offspring was then screened for sex and kept to inbreed to check for any sign of fertility of potential not-recognized $F_{1}$ females. All inspected $F_{1}$ progeny was male or intersex and no $\mathrm{F}_{2}$ progeny was observed. To estimate the homing efficiency of the $\operatorname{tra}^{n C H E}, 12$ heterozygous $\operatorname{tra} a^{n C H E} /+$ males were individually crossed with three $W^{-}$virgins each. The offspring was then screened for sex and the presence of DsRed fluorescent eye marker under a Zeiss fluorescent binocular (Fig. S3 $B$ and $C$ ). The homing efficiency was calculated as the ratio of targetable alleles that had been successfully homed by the $\operatorname{tra}^{n C H E}$ allele, by equation E11, assuming an equal segregation of chromosomes in male gametes.

$$
\frac{\text { DsRed }^{+}-(\text {Total } / 2)}{\text { Total/2 }}
$$

Estimating the targeting efficiency. To estimate the targeting efficiency of our $\mathrm{CHE}$, heterozygous $\operatorname{tra}^{n C H E} /+$ males were crossed with heterozygous $\operatorname{tra}^{n D O C K} /+$ virgins (Fig. 2) - both in batch and in single crosses with individual $\operatorname{tra}^{n C H E}$ males. The $\mathrm{F}_{1}$ offspring was then screened for eye marker and only individuals carrying the ECFP eye marker but not the DsRed eye marker were selected for molecular analysis. MK058/MK059 primer pairs (Table S1) were used to amplify a 750 bp region which was used for Sanger sequencing using the MK058 primer. Sequencing results were analyzed using Geneious software (Biomatters, New Zealand). Templates were prepared by homogenizing the head of individual flies in $50 \mu \mathrm{l}$ of smashing buffer (149) using small tips.

Population experiments. Population experiments were performed in four settings A-D with five replicates for each of the settings (Fig. S6 A). To avoid any bias in mating, we made sure all flies were of the same age and before putting them together in the same vial all flies were kept anesthetized using $\mathrm{CO}_{2}$. After 72 hours of mating time, a 3-6 hours egg lay collection was obtained, after which all parents were removed and were kept frozen at $-80^{\circ} \mathrm{C}$. For each following generation, all progeny of the 3-6 hour egg collection was allowed to eclose and then used as parents to set up the next generation. Again after a 3-6 hours egg lay, these parents were removed and used for screening their sex and the presence 
of the DsRed eye marker prior to freezing and storage at $-80^{\circ} \mathrm{C}$. The $3-6$ hour egg collection was based on the amount of time that is necessary for the population of parents to lay enough eggs to generate a progeny size which fills but not over-populates a medium size fly food vial (Diameter: 36 $\mathrm{mm}$; Height: $82 \mathrm{~mm}$ ). At each generation, enough time was given to all the pupae to eclose followed by 72 hours of mating to allow for a representative population in the next generation. For each egg collection, all parents as well as all the respective progeny were screened.

Molecular analysis of tra loci and aborted HDR tra alleles. MK058 and MK059 primers (Table S1) were used to PCR-amplify a $750 \mathrm{bp}$ fragment surrounding the $\mathrm{g} 4$ target site. Templates were prepared by homogenizing the head of individual flies in $50 \mu \mathrm{l}$ of smashing buffer (149) using small tips. Samples were kept at $55^{\circ} \mathrm{C}$ for 1 hour followed by 5 minutes incubation at $95^{\circ} \mathrm{C}$ to heat inactivate the Proteinase K. $5 \mu \mathrm{l}$ of samples were used for PCR using Phusion DNA polymerase in $50 \mu \mathrm{l}$ by 2 minutes incubation at $96^{\circ} \mathrm{C}$ followed by 40 cycles of $96^{\circ} \mathrm{C}$ for $20^{\prime \prime}, 70^{\circ} \mathrm{C}$ for $30^{\prime \prime}$, and $72^{\circ} \mathrm{C}$ for $40^{\prime \prime}$ with 5 minutes final extension at $72^{\circ} \mathrm{C}$. PCR products were then sent for Sanger sequencing using primer MK058. Sequencing results were analyzed using Geneious software (Biomatters, New Zealand). MK146 and MK134 primers (Table S1) were used to PCR amplify a $\sim 6 \mathrm{kbp}$ fragment consisting of the U6:3-g4 and SpCas9 CDS from DsRed female's genomic DNA. PCR products were analyzed by agarose gel electrophoresis and fragment sizes were compared with the PCR product of same primer pairs on genomic DNA of a male individual from the stock as reference. The reduction in size of the fragments served as an indication of an aborted HDR (Fig. S4 $A$ ). To confirm the results at the molecular level, PCR products were then used for Sanger sequencing using MK085, MK086, MK087, and MK088 primers (Table S1).

Population genetic model for homing system targeting the tralocus in C. capitate. To model the potential application of the homing-based gene drive system targeting the tra locus to suppress populations of the medfly, C. capitata, we combined a population genetic model describing the inheritance pattern of the homing system (Fig. S10) with a population dynamic model described previously (163) adapted for the C. capitata (the original model describes the population dynamics of the main African malaria vector, An. gambiae) (Fig. S11).

In the population genetic model (Fig. S10), we describe the offspring genotype distribution in $C$. capitata for each combination of maternal and paternal genotypes for a homing-based gene drive system targeting the tra locus. We denote the wild-type tra allele by "W", the drive allele, $\operatorname{tra}^{\mathrm{nCHE}}$, by "D", a drive-resistant allele with an in-frame indel at the tralocus, $\operatorname{tra}^{\mathrm{Rst}}$, by " $\mathrm{R}$ ", and a drive-resistant null allele, tra', by "B". Homing is only manifest in WD heterozygotes, whereby WD individuals produce $\mathrm{D}$ gametes in the germline at a frequency equal to $(1+e) / 2$, where $e$ denotes the "homing efficiency," which represents the proportion of W gametes that are converted into D gametes through the act of homing. Homing efficiency, $e$, is equal to the product of the cleavage rate, $c$, and the probability of accurate homology-directed repair (HDR) given that cleavage has occurred, $P_{H D R}$, i.e. $e=c P_{H D R}$. Wild-type alleles may also be produced by WD individuals at a rate equal to $(1-c) / 2$; however, it should be noted that the rate at which this occurs has been shown to be negligible in a recent analysis (164) and hence this possibility is not depicted in the crosses in Fig. S10. Homingresistant alleles, which result from $\mathrm{W}$ alleles that are cleaved but for which accurate HDR does not occur, are produced at a frequency equal to $c \delta / 2$, where $\delta=\left(1-P_{H D R}\right)$. A proportion, $\theta$, of these resistant 
alleles are in-frame indels $(\mathrm{R})$, for which expression of functional tra gene is retained, and the remainder, $(1-\theta)$, are out-of-frame or other mutations $(\mathrm{B})$, for which the function of tra gene is disrupted. The proportion of $\mathrm{W}$ gametes that are converted to $\mathrm{R}$ or $\mathrm{B}$ gametes is therefore given by $\rho_{\mathrm{R}}=c \delta \theta$ and $\rho_{\mathrm{B}}=c \delta(1-\theta)$, respectively. The proportion of generated resistant alleles that are in-frame indels is expected to be $\sim 1 / 3$, although this proportion can be reduced through multiplexing. All other individuals produce gametes at standard Mendelian frequencies. In this system, there are six possible fertile female genotypes - DR/XX, RR/XX, RB/XX, WR/XX, WB/XX and WW/XX - and 14 possible fertile male genotypes - DD/XY, WD/XY, DR/XY, DB/XY, RR/XY, RB/XY, BB/XY, WR/XY, WB/XY, WW/XY, DD/XX, WD/XX, DB/XX and BB/XX (Fig. S10).

Population dynamic model for $\boldsymbol{C}$. capitate. In the adapted population dynamics model (Fig. S11), the medfly life cycle is divided into four life stages - egg, larva, pupa and adult (both male and female adults are modeled) (Fig. S10). The durations of the juvenile stages differ (Table S2) but their daily, density-independent mortality rates are assumed to be identical and are chosen for consistency with the population growth rate in the absence of density-dependent mortality. The intrinsic population growth rate of medfly populations in the absence of density-dependent mortality, $r_{M}$, was calculated from average monthly trap catch data across four sites in Western Cape Province, South Africa between the months of December 2009 and March 2010 and shown to be consistent with a population growth rate of $r_{M}=1.031$ per day (165). Additional density-dependent mortality occurs at the larval stage, and we used a density-dependent equation of the form, $F(L)=\sqrt[T_{L}]{(+L)}$, where $L$ is the number of larvae, $T_{L}$ is the duration of the larval stage, and $\alpha$ is a parameter influencing the strength of densitydependence. Adult males mate throughout their lifetime, while adult females mate only once, soon after that they mature. For these simulations, we assumed fecundity rates to be consistent between genotypes, with fertile females laying $\beta$ eggs per day. Initial estimates for these and other parameter values are provided in Table S2 and the equations describing the equivalent implementation of this model for An. gambiae are included in Supplementary File S1 of (163).

We used a stochastic implementation of this model to capture random effects at low population sizes, for instance when the CRISPR-based homing system is causing significant population suppression. We assume the number of eggs produced per day by females follows a Poisson distribution, the number of eggs having each genotype follows a multinomial distribution according to the parental genotypes and inheritance pattern, and all survival/death events follow a Bernoulli distribution. Finally, female mate choice follows a binomial distribution with probabilities given by the relative frequency of each male genotype in the population.

Experimental design and statistics. All individual data points are displayed in supplementary figures with mean and s.d., and sample size for all experiments are mentioned in the main text and figures as appropriate. No power calculations were performed to estimate the sample size. No randomization or blinding was performed, for each cross all progeny was screened. Replicate numbers for estimation of efficiencies are consistent with other similar gene drive studies in insects. A normal probability plot was generated for obtained homing efficiencies of each replicate to ensure the normal distribution of data points. In crosses with individual heterozygous $\operatorname{tra}^{n C H E}$ flies, replicates that did not result in progenies were excluded. These were most likely a result of a cross between an infertile intersex fly 
which are often indistinguishable from true males. In crosses between individual heterozygous $\operatorname{tra} a^{n C H E}$ males with heterozygous $\operatorname{tra}^{n D O C K}$ virgins, two of the replicates that showed a $1: 1$ ratio of DsRed $^{+}$:DsRed ${ }^{-}$offspring were not used for molecular analysis and sequencing. All statistical analyses were performed on Microsoft Excel 2007. Plots were generated using GraphPad Prism 7 and R. 


\section{SI Figures}

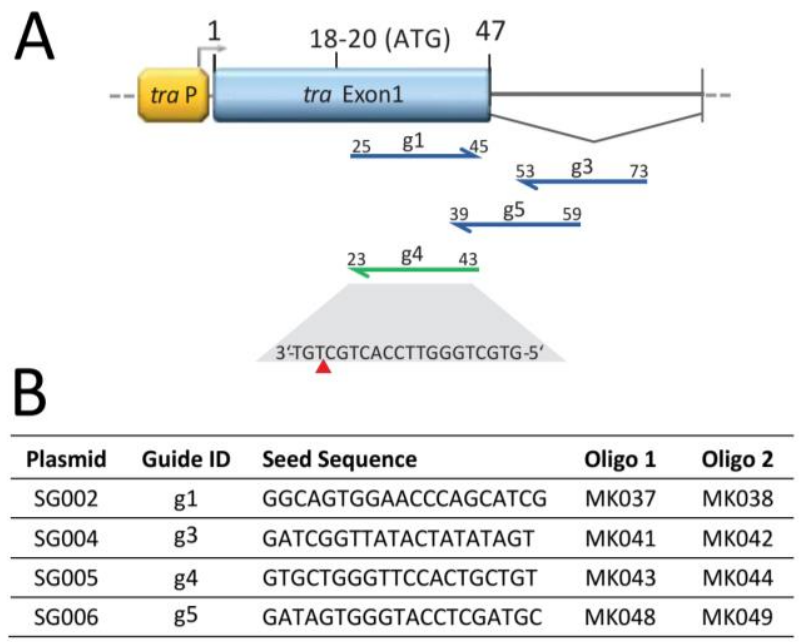

C

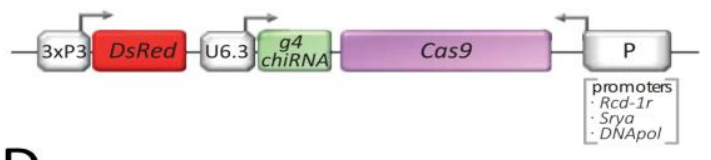

D

\begin{tabular}{|c|c|c|c|c|}
\hline \multicolumn{5}{|c|}{ Female Offspring [\%] } \\
\hline Replicate & $\begin{array}{l}\text { tra }{ }^{\mathrm{nDOCK}} \\
\text { Control }\end{array}$ & \multicolumn{3}{|c|}{ Promoter in tra ${ }^{\mathrm{nCSE}}$} \\
\hline 1 & 56.14 & 20.76 & 20.33 & 3.96 \\
\hline 2 & 54.60 & 23.11 & 15.85 & 20.49 \\
\hline 3 & 49.11 & 12.76 & 8.99 & 4.27 \\
\hline 4 & 51.18 & 10.79 & 22.22 & 17.68 \\
\hline 5 & 53.51 & 2.34 & 10.40 & 7.77 \\
\hline 6 & 52.78 & 15.27 & 24.39 & 14.83 \\
\hline 7 & 49.30 & 11.22 & 0 & 8.61 \\
\hline 8 & 42.22 & 14.06 & 0 & 15.00 \\
\hline 9 & 45.08 & 0 & 14.62 & 10.24 \\
\hline 10 & 39.07 & 17.18 & N/A & 30.09 \\
\hline Average & 49.30 & 12.75 & 12.98 & 13.29 \\
\hline
\end{tabular}

Fig. S1. Generation of a homing CHE for the D. melanogaster tra locus I. (A) Four gRNAs targeting the first exon of the D. melanogaster tra locus with $\mathrm{g} 4$ (green) identified as the most efficient gRNA using HRMA (numbers indicate bases after transcription start site). (B) Oligos (Table S1) used for the generation of gRNA plasmids. (C) Structure of the CHE used in this study. Human codon optimized $\operatorname{Sp} \operatorname{Cas} 9$ coding sequence is placed under the control of each of the three promoters under study. Rcd-1r germline specific promoter, Sry- $\alpha$ early zygotic promoter, and DNApol- $\alpha 180$ cell cycle dependent promoter. g4 guide RNA is expressed by $U 6: 3$ promoter. A $3 \mathrm{xP} 3$ driven DsRed eye marker is used to allow for screening and identification of strains. (D) To evaluate the somatic sex conversion efficiency of each of the promoters, individual $t r a^{\mathrm{nCHE}}$ males from each of the three CHE variants ( $R c d-1 r$-CHE, Sry- $\alpha$-CHE, DNApol- $\alpha 180-\mathrm{CHE}$ ) were crossed with $w$ - virgins and offspring were screened for their sex. $\operatorname{tra}^{\mathrm{nDOCK}}$ strain was used as control. The very low frequency of female offspring clearly indicates that all three of these promoters are capable of inducing somatic sex conversion in D. melanogaster. Assuming an equal distribution of $\mathrm{X}$ and $\mathrm{Y}$ chromosomes and a sex conversion rate of $100 \%$ in XX embryos receiving a tra ${ }^{\mathrm{n}} \mathrm{CHE}$ allele from their father, a homing rate of $\sim 49 \%$ can be calculated for each of these promoters from the below equation which indicates all three of these promoters had equally well been able to successfully perform homing in the germline of the heterozygous $\operatorname{tra}^{\mathrm{n}} \mathrm{CHE}$ males and drive into the next generation.

Homing efficiency $=\frac{E_{f}^{\prime}-O_{f}}{E_{f}^{\prime}}$ 


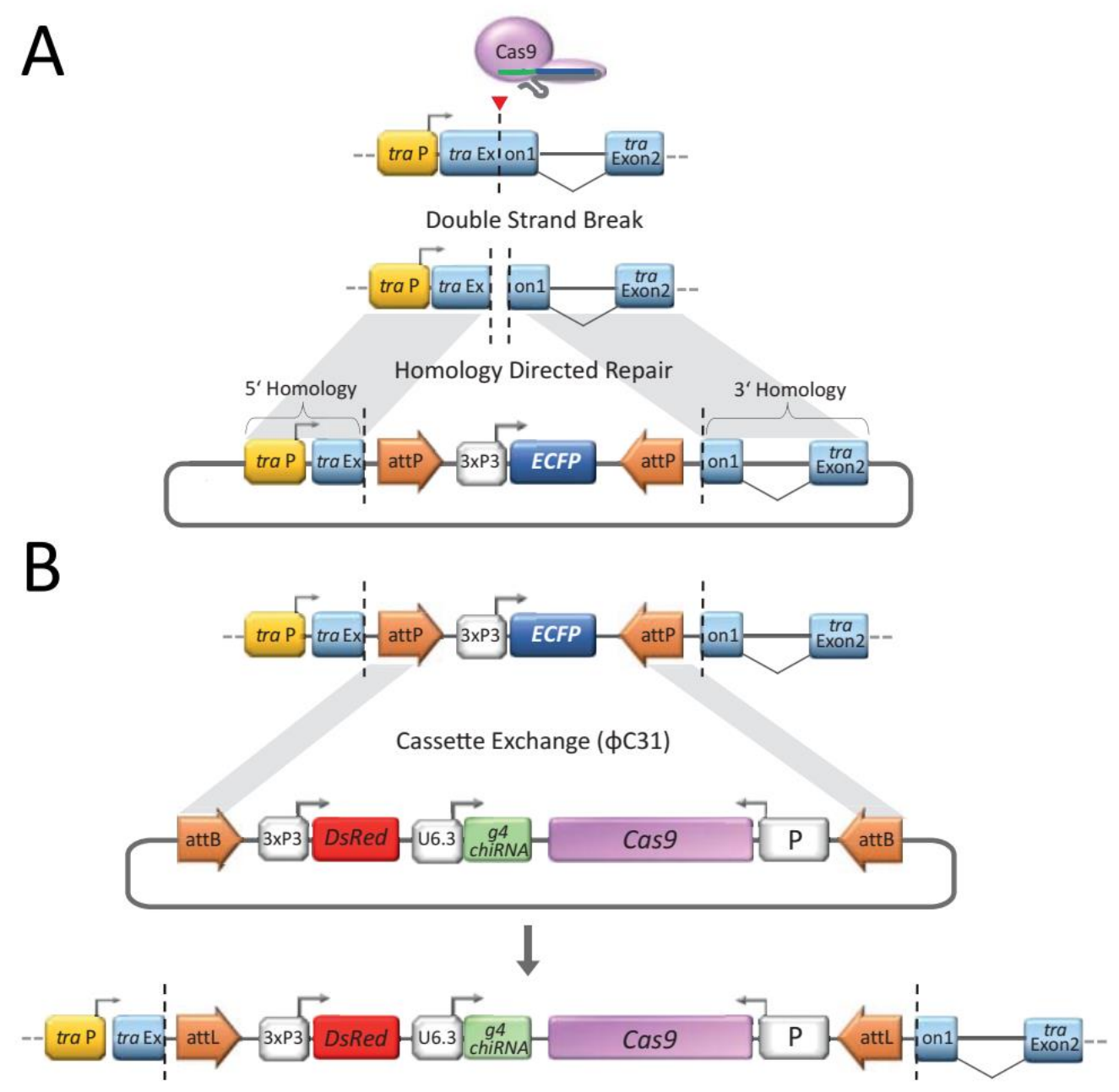

Fig. S2. Generation of a homing CHE for the D. melanogaster tra locus II. (A) Using the g4 gRNA and Cas9, a ФC31 RMCE docking cassette containing an eye-specifically driven (3xP3) cyan fluorescent marker (ECFP) flanked by attP sites was integrated into the 1 st exon of the tra locus to generate the docking null allele $t r a^{n D O C K}$. (B) A cassette - containing the coding sequence of SpCas9 endonuclease (Cas9, under the control of $R c d-1 r$ germline specific promoter), the U6.3 driven chimeric gRNA ( $g 4$ chiRNA), and a red fluorescent eye marker (DsRed) - was used to replace the ECFP marker by $\Phi C 31$-mediated RMCE to generate the homing CHE null allele $\operatorname{tra}^{n C H E}$. 
A

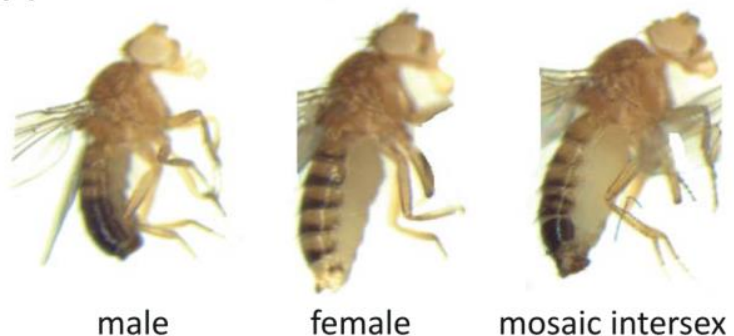

B

\begin{tabular}{|c|c|c|c|c|c|c|c|}
\hline \multirow{2}{*}{$\begin{array}{l}\text { Total No. } \\
\text { Flies }\end{array}$} & \multicolumn{2}{|c|}{ Male } & \multicolumn{2}{|c|}{ Female } & \multirow{2}{*}{$\begin{array}{c}\text { Male } \\
\text { Frequency }\end{array}$} & \multirow{2}{*}{$\begin{array}{l}\text { DsRed }^{+} \\
\text {Frequency }\end{array}$} & \multirow{2}{*}{$\begin{array}{l}\text { Homing } \\
\text { Efficiency }\end{array}$} \\
\hline & DsRed $^{+}$ & DsRed & DsRed $^{+}$ & DsRed' & & & \\
\hline 238 & 221 & 8 & 0 & 9 & $96.22 \%$ & $92.86 \%$ & $85.71 \%$ \\
\hline 183 & 155 & 13 & 0 & 15 & $91.80 \%$ & $84.70 \%$ & $69.40 \%$ \\
\hline 111 & 93 & 8 & 0 & 10 & $90.99 \%$ & $83.78 \%$ & $67.57 \%$ \\
\hline 158 & 129 & 11 & 0 & 18 & $88.61 \%$ & $81.65 \%$ & $63.29 \%$ \\
\hline 158 & 126 & 17 & 0 & 15 & $90.51 \%$ & $79.75 \%$ & $59.49 \%$ \\
\hline 204 & 162 & 23 & 0 & 19 & $90.69 \%$ & $79.41 \%$ & $58.82 \%$ \\
\hline 184 & 144 & 18 & 0 & 22 & $88.04 \%$ & $78.26 \%$ & $56.52 \%$ \\
\hline 195 & 150 & 24 & 0 & 21 & $89.23 \%$ & $76.92 \%$ & $53.85 \%$ \\
\hline 214 & 164 & 25 & 0 & 25 & $88.32 \%$ & $76.64 \%$ & $53.27 \%$ \\
\hline 206 & 147 & 34 & 0 & 25 & $87.86 \%$ & $71.36 \%$ & $42.72 \%$ \\
\hline 161 & 112 & 30 & 0 & 19 & $88.20 \%$ & $69.57 \%$ & $39.13 \%$ \\
\hline 240 & 149 & 32 & 0 & 59 & $75.42 \%$ & $62.08 \%$ & $24.17 \%$ \\
\hline Average & & & & & $88.82 \%$ & $78.08 \%$ & $56.16 \%$ \\
\hline Standard & aon & & & & $4.83 \%$ & $7.91 \%$ & $15.83 \%$ \\
\hline
\end{tabular}

C

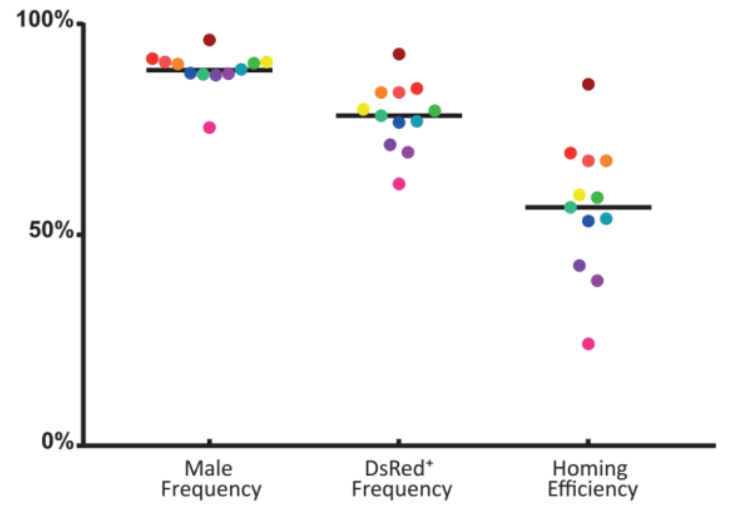

Fig. S3. Sex Conversion and Homing Efficiency. (A) Mosaic intersex fly (right). Because of the high efficiency of Cas 9 at cleaving wild-type tra alleles also in somatic cells, heterozygous $t r a^{n C H E} /+\mathrm{XX}$ individuals develop into infertile mosaic intersex individuals. These intersex individuals, often lack sex combs on one or both of their front legs, show a larger size, show a malformed ovipositor and have an inconsistent yellow and black pattern on their abdomen. (B) Estimation of the homing efficiency at the tra locus. Twelve $t r a^{n C H E} /+$ males were crossed individually with virgin $w^{-}$flies and progenies were screened for sex and presence of the DsRed eye marker. On average about $78 \%$ of the offspring carried the DsRed marker as opposed to the expected $50 \%$ based on Mendelian inheritance. The homing efficiency was calculated using the formula $\frac{\text { DsRed }{ }^{+}-(\text {Total } / 2)}{\text { Total } / 2}$ and determined to be around 56\%. (C) Dot plots showing the distribution of male frequency, DsRedfrequency, and homing efficiency values of the twelve replicates (colors represent the different replicates as indicated in panel B). 

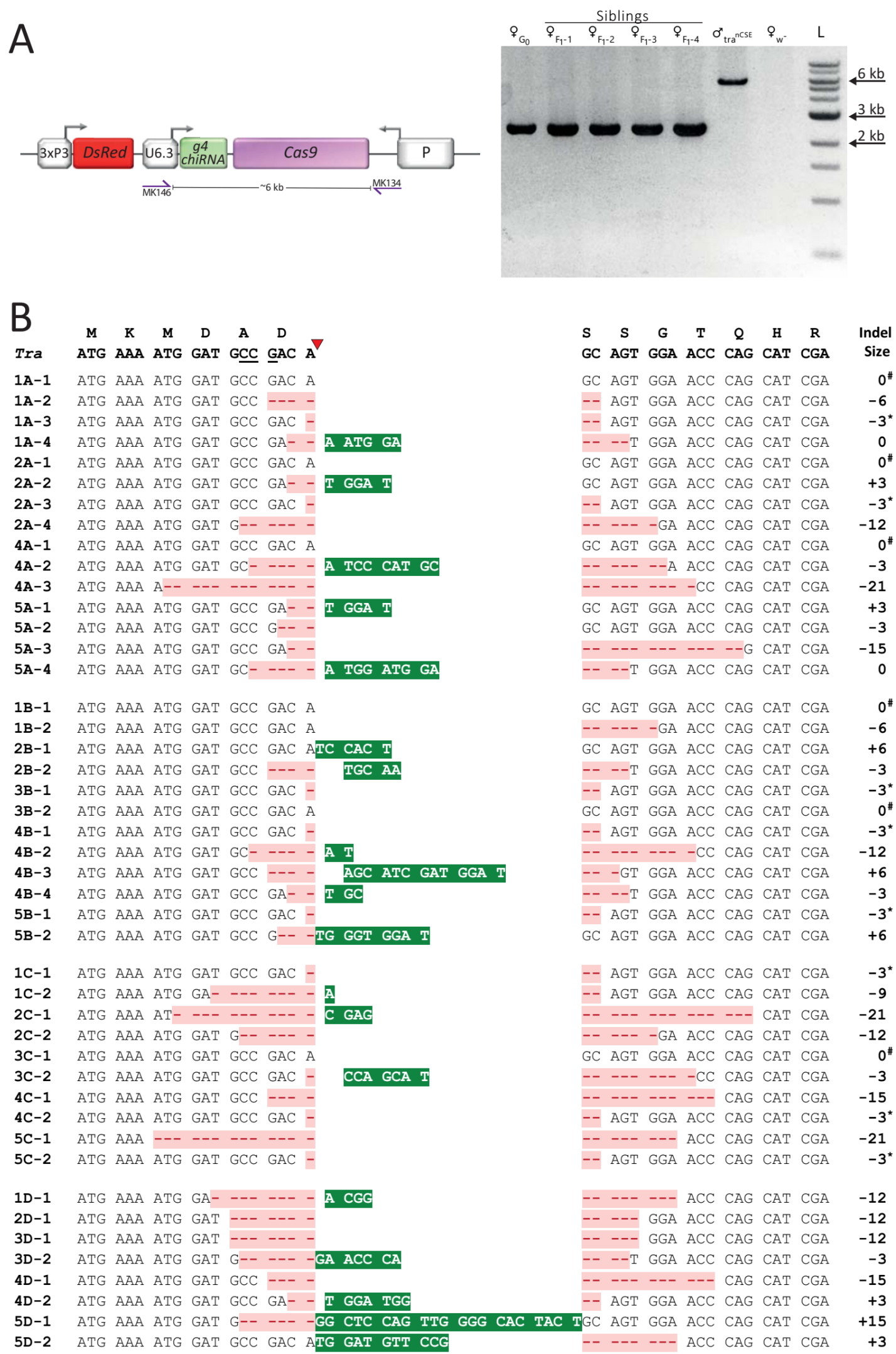

Fig. S4. Example of aborted HDR and CHE-resistant tra alleles in generation $\mathrm{F}_{6}$ of the population experiments. (A) Aborted HDR: In one of the crosses of females with the DsRed eye marker no signs of an active drive system was observed. Amplification of the Cas9 expression cassette using MK134/MK146 primer pair should result into a 6 Kb DNA fragment (Third lane from right; $\operatorname{tra}^{\mathrm{nCHE}}$ ). However, PCR on the genomic DNA of the mother and its female offspring that 
carried the DsRed eye marker with these primers resulted in a truncated $\sim 2.5 \mathrm{~Kb}$ product, indicating a large deletion in the $\mathrm{CHE}$ as a result of an aborted HDR event. (B) Molecular analysis of the CHE target site sequences in DsRed-marked females derived from all replicates (first number) of all four setting A-D (letter). The last number indicates different sequences from the same replicate. Few of the sequences of Setting A were obtained from $F_{13}$, as DsRed females were absent in $\mathrm{F}_{6}$ of some replicates. The unchanged wild type tra allele was identified in six occasions (indel size: $\mathrm{O}^{\#}$ ), but those individuals had defective $\operatorname{tra}^{n C H E}$ alleles with large deletions in the Cas 9 coding sequence (not shown) as the result of an aborted HDR (panel A). In all other DsRed females, $\operatorname{tra}^{\mathrm{Rst}}$ mutations were found that represent in-frame indels, which have, at least, either the seed sequence of the target site or the CRISPR protospacer adjacent motif (PAM) abolished. The size of the indels were multiples of three keeping the tra coding sequence in-frame but destroying the g4 gRNA's recognition sequence at the cleavage site (indicated by a red triangle). The diversity of the indels indicates frequent independent events that can result in the emergence of resistant alleles. A three-base pair AGC deletion at the site of cleavage was identified in eight independent occasions $\left(-3^{*}\right)$. 


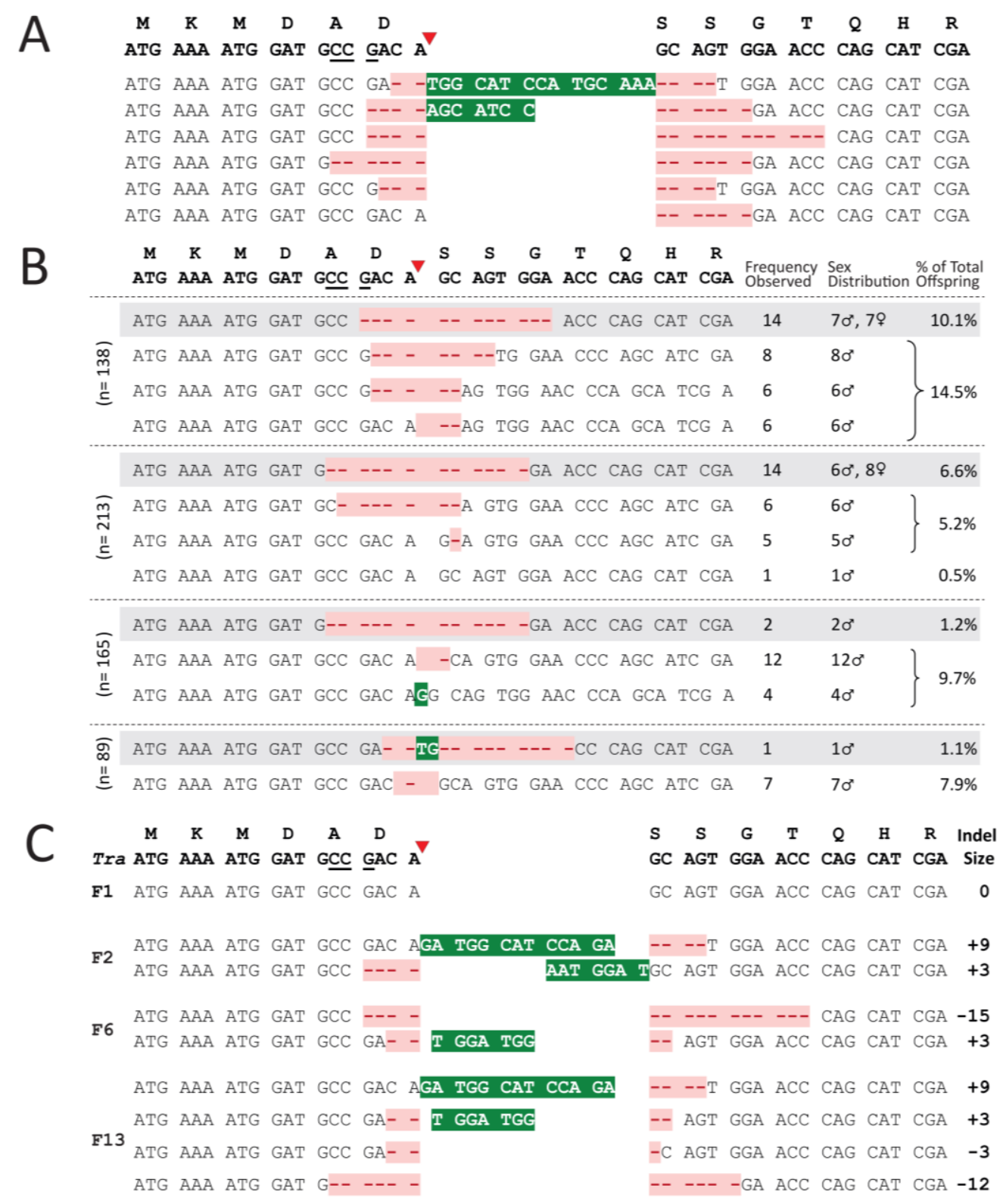

Fig. S5. Molecular analysis of non-HDR events at the site of cleavage. (A) Molecular analysis of the CHE target site (red triangle indicates cleavage site) in $\mathrm{F}_{1}$ female progeny of a cross between $\operatorname{tra} a^{n C H E} /+$ males and $\operatorname{tra} a^{n D O C K} /+$ virgins (Fig. 2) identified independent NHEJ events causing various in-frame indels (red/green) that resulted in resistant alleles. (B) Analysis of all ECFP/non-DsRed progeny derived from four individually crossed $t r a^{n C H E} /+$ males and $t r a^{n D O C K} /+$ virgins (separated by dashed lines) indicates the very efficient targeting of the tra locus in the presence of an active homing CHE (only one wild type allele in a progeny of 605). NHEJ-derived alleles were identified in 9-24\% of all progeny causing frameshift mutations $(t r a)$ in about two thirds of these cases and in-frame indels ( $\operatorname{tra}^{\mathrm{Rst}}$, shaded in grey) in about one third, whereby $\operatorname{tra}^{-}$alleles were expectedly only observed in males or intersexes. The limited number of different NHEJ-derived alleles per single male cross indicates an activity at very early stages of primordial germ cell development. (C) Molecular analysis of the CHE target site (red triangle indicates cleavage position) in the tra locus of population experiment-derived DsRed-marked females. All sequences are taken from flies of the fourth replicate of setting D (SI Appendix, Fig. S4) across different generations. DsRedmarked females from $\mathrm{F}_{1}$ carried a wild type tra allele but defective $\operatorname{tra}^{n}{ }^{C H E}$ alleles with large deletions in the Cas 9 coding sequence as a result of an aborted HDR (Fig. 2). In $\mathrm{F}_{2}$ and $\mathrm{F}_{6}$, various in-frame indel mutations were identified indicating independent emergences of these alleles. Some alleles already observed in $F_{2}$ and $F_{6}$ were also isolated in $F_{13}$, which implies the spread and fixation of these resistant alleles in the population. 
A

\begin{tabular}{lcccc}
\hline Setting & $\begin{array}{c}\text { No. of } \\
\text { Replicates }\end{array}$ & $\begin{array}{c}\text { No. of } \\
w^{-} \text {Virgins }\end{array}$ & $\begin{array}{c}\text { No. of } \\
w^{-} \text {Males }\end{array}$ & $\begin{array}{c}\text { No. of } \\
\text { tra }^{\text {CSEE }} \text { Males }\end{array}$ \\
\hline $\mathrm{A}(1: 10)$ & 5 & 30 & 30 & 3 \\
\hline $\mathrm{B}(1: 2)$ & 5 & 30 & 30 & 15 \\
\hline $\mathrm{C}(1: 1)$ & 5 & 30 & 30 & 30 \\
\hline $\mathrm{D}(1: 0)$ & 5 & 30 & 0 & 30 \\
\hline
\end{tabular}

B

Male Frequency

( $\sigma^{\prime \prime} \sigma^{r} /$ Total)

DsRed $^{+}$Frequency

(DsRed ${ }^{+} /$Total)

Resistance Allele Indicator

(DsRed + $\%$ /\% $\%)$
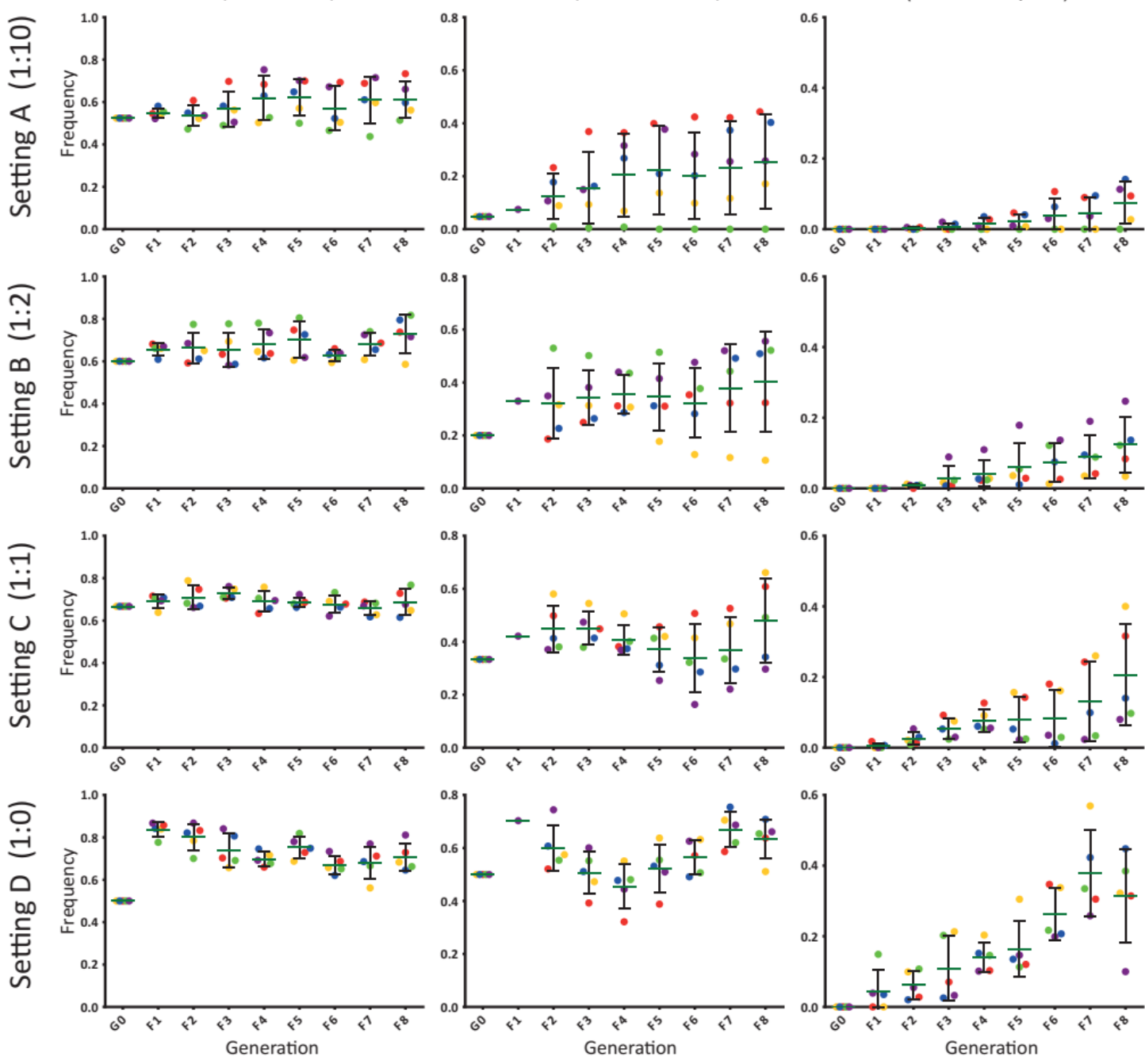

- Replicate $1 \bullet$ Replicate $2 \bullet$ Replicate $3 \bullet$ Replicate $4 \bullet$ Replicate 5

Fig. S6. Population experiments. (A) Set-ups of the different cage experiments (settings A-D). Values in parentheses show the ratio of carrier $t r a^{n C H E}$ males to $W^{-}$males used in each setting. (B) Dot plots representing the results of all five replicates (each indicated by different colors) for each setting of the population experiments. The increase in the DsRed females over the generations is an indicator for the emergence and spread of resistant alleles. Data for the DsRed frequency in $F_{1}$ is from replicate five only (purple dots). In setting A, replicate three (green dots) lost its $\operatorname{tra}^{n C H E}$ allele from $\mathrm{F}_{2}$ onwards. 


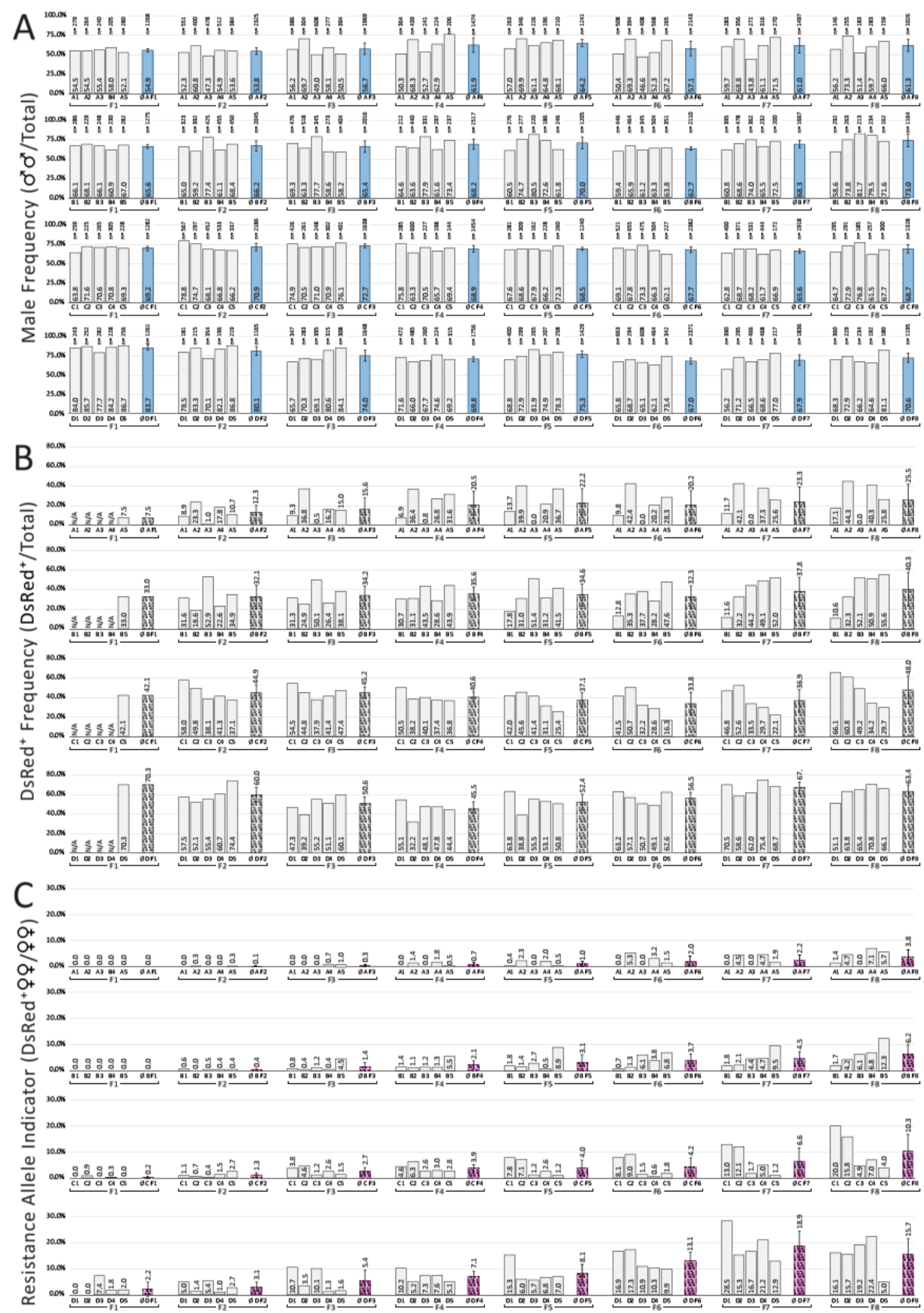

Fig. S7. Original data of the population experiments. Five replicates for each of the four settings A-D (Fig. S6A) were monitored over eight generations $\left(\mathrm{F}_{1}-\mathrm{F}_{8}\right)$. (A) The frequency of males in the respective population. (B) The DsRed frequency depicting the percentage of flies carrying the DsRed eye marker (in $F_{1}$ data only available for replicate five). (C) The resistance allele indicator represented by the proportion of females that carry the DsRed eye marker. Color/pattern-marked columns represent mean and standard deviation for each setting and generation. These means were taken to produce Fig. $3 A$ (please note, for the DsRed frequency in $F_{1}$, only replicate five was counted and that value taken as representative). 
A

Setting B (1:2)

\begin{tabular}{ccccccccc}
\hline \multirow{2}{*}{$\#$} & $\begin{array}{c}\text { Total No. } \\
\text { Flies }\end{array}$ & \multicolumn{2}{c}{ Male } & \multicolumn{2}{c}{ Female } & $\begin{array}{c}\text { Male } \\
\text { nsequed }\end{array}$ & $\begin{array}{c}\text { DsRed }^{+} \\
\text {Frequency }^{+}\end{array}$ & $\begin{array}{c}\text { Resistance Allele } \\
\text { Indicator }\end{array}$ \\
\hline 1 & 337 & 98 & 68 & 54 & 117 & $49.26 \%$ & $29.08 \%$ & $31.58 \%$ \\
2 & 413 & 106 & 102 & 105 & 100 & $50.36 \%$ & $25.67 \%$ & $51.22 \%$ \\
3 & 347 & 129 & 53 & 101 & 64 & $52.45 \%$ & $37.18 \%$ & $61.21 \%$ \\
4 & 381 & 146 & 55 & 107 & 73 & $52.76 \%$ & $38.32 \%$ & $59.44 \%$ \\
5 & 454 & 190 & 72 & 114 & 78 & $57.71 \%$ & $41.85 \%$ & $59.38 \%$ \\
\hline Average & & & & & & $\mathbf{5 2 . 5 1 \%}$ & $\mathbf{3 4 . 4 2 \%}$ & $\mathbf{5 2 . 5 7 \%}$ \\
Standard Deviation & & & & & $\mathbf{3 . 2 5 \%}$ & $\mathbf{6 . 7 7 \%}$ & $\mathbf{1 2 . 3 6 \%}$
\end{tabular}

C

Setting $D$ (1:0)

\begin{tabular}{cccccc}
\hline \multirow{2}{*}{$\#$} & \multirow{2}{*}{$\begin{array}{c}\text { Total No. } \\
\text { Flies }\end{array}$} & \multicolumn{2}{c}{ Male } & \multicolumn{2}{c}{ Female } \\
\cline { 3 - 6 } & DsRed $^{+}$ & DsRed $^{-}$ & DsRed $^{+}$ & DsRed \\
\hline 1 & 446 & 169 & 80 & 100 & 97 \\
2 & 327 & 121 & 62 & 77 & 67 \\
3 & 437 & 101 & 97 & 118 & 121 \\
4 & 432 & 109 & 99 & 113 & 11 \\
5 & 434 & 126 & 139 & 53 & 11
\end{tabular}

Average

Standard Deviation

$3.25 \%$

$6.77 \%$
B

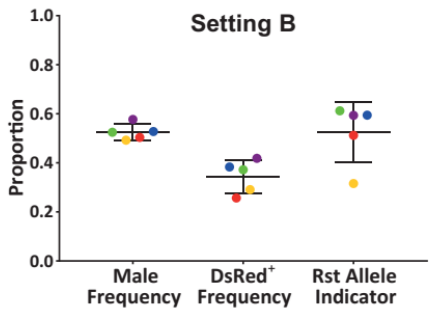

D

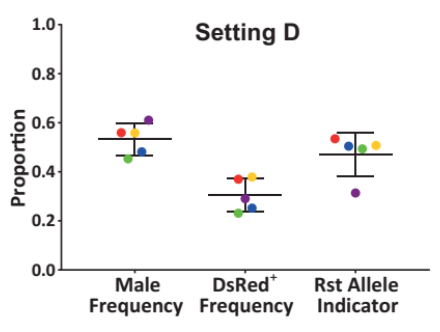

Fig. S8. Population collapse experiment data for generation $\mathbf{F}_{\mathbf{1 5}}$. (A, B) Setting B and (C, D) Setting D populations recovered from the masculinizing effect of the $\operatorname{tra}^{n C H E}$ homing allele as the sex ratios are back to around normal $50 \%$. The DsRed eye marker was present in about one third of the population but in almost half of the females serving as resistance allele indicator. Original data $(\mathrm{A}, \mathrm{C})$ and dot plot representation $(\mathrm{B}, \mathrm{D})$ of all five replicates (each indicated by a different color). The averages were taken to produce Fig. $3 \mathrm{~A}$. 


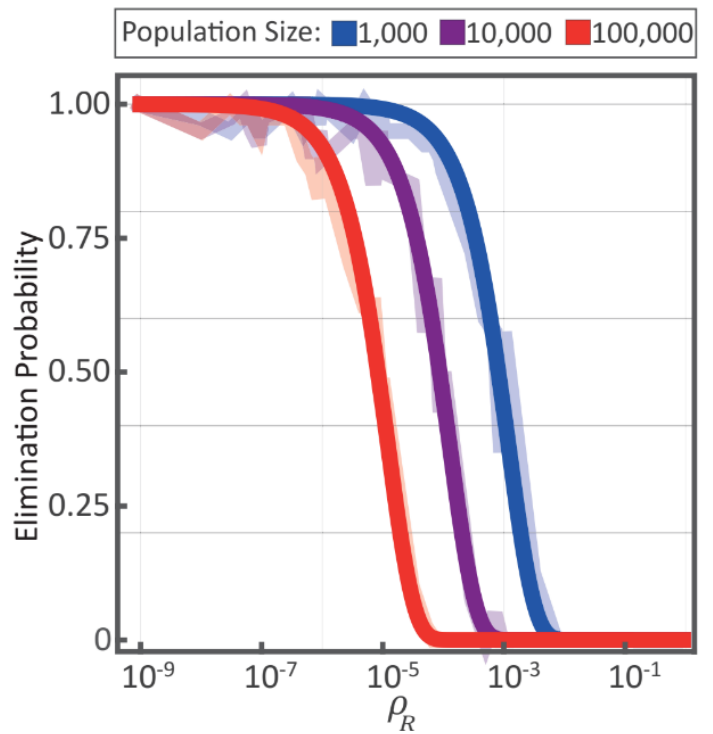

Fig. S9. Elimination probability as a function of in-frame resistant allele generation rate, $\rho_{R}$, for a range of population sizes, $\boldsymbol{N}$, between 1,000 and 100,000 . We consider the scenario in which $\operatorname{tra}^{n C H E}$; XX individuals are fertile males, and assume a 1:1 release to wild ratio, that CRISPR-mediated cleavage efficiency is $100 \%$, that the probability of accurate homology-directed repair following cleavage is $90 \%$, that the in-frame resistant allele generation rate is as specified above, that in-frame resistant alleles have no associated fitness cost, and that the remainder of resistant alleles are out-of-frame or other mutations. Sigmoidal curves are fitted to data points covering $30 \mathrm{in}$-frame resistant allele generation rates sampled logarithmically between $10^{-1}$ and $10^{-9}$. Faint lines represent interpolation between simulated data points while solid lines represent fitted sigmoidal relationships. For an adult population size of 1,000, an in-frame resistant allele generation rate of less than $10^{-4}$ is required to achieve likely elimination, while for an adult population size of 100,000, an in-frame resistant allele generation rate of less than $10^{-6}$ is required to achieve likely elimination. 
Female

\begin{tabular}{|c|c|c|c|c|c|c|}
\hline & $D R / X X$ & $R R / X X$ & $R B / X X$ & WR/XX & WB/XX & $W W / X X$ \\
\hline $\mathrm{DD} / \mathrm{XY}$ & $\begin{array}{l}(1 / 2) D R \\
(1 / 2) D D\end{array}$ & (1) $\mathrm{DR}$ & $\begin{array}{l}(1 / 2) D R \\
(1 / 2) D B\end{array}$ & $\begin{array}{l}(1 / 2) W D \\
(1 / 2) D R\end{array}$ & $\begin{array}{l}(1 / 2) W D \\
(1 / 2) D B\end{array}$ & (1) WD \\
\hline WD/XY & $\begin{array}{l}((1+e) / 4) D D \\
\left(\left(1+e+\rho_{R}\right) / 4\right) \\
D R \\
\left(\rho_{R} / 4\right) R R \\
\left(\rho_{g} / 4\right) D B \\
\left(\rho_{B} / 4\right) \text { RB } \\
\end{array}$ & $\begin{array}{c}((1+\mathrm{e}) / 2) \mathrm{DR} \\
\left(\rho_{\mathrm{R}} / 2\right) \mathrm{RR} \\
\left(\rho_{\mathrm{B}} / 2\right) \mathrm{RB}\end{array}$ & $\begin{array}{c}((1+\mathrm{e}) / 4) \mathrm{DR} \\
((1+\mathrm{e}) / 4) \mathrm{DB} \\
\left(\rho_{\mathrm{R}} / 4\right) \mathrm{RR} \\
\left(\left(\rho_{\mathrm{R}}+\rho_{\mathrm{B}}\right) / 4\right) \mathrm{RB} \\
\left(\rho_{\mathrm{B}} / 4\right) \mathrm{BB} \\
\end{array}$ & $\begin{array}{c}((1+\mathrm{e}) / 4) W D \\
((1+\mathrm{e}) / 4) \mathrm{DR} \\
\left(\rho_{\mathrm{g}} / 4\right) \mathrm{WR} \\
\left(\rho_{\mathrm{p}} / 4\right) \mathrm{RR} \\
\left(\rho_{\mathrm{g}} / 4\right) \mathrm{WB} \\
\left(\rho_{\mathrm{g}} / 4\right) \mathrm{RB} \\
\end{array}$ & \begin{tabular}{|c|}
$((1+\mathrm{e}) / 4) W D$ \\
$((1+\mathrm{e}) / 4) \mathrm{DB}$ \\
$\left(\rho_{\mathrm{g}} / 4\right) W R$ \\
$\left(\rho_{\mathrm{g}} / 4\right) \mathrm{RB}$ \\
$\left(\rho_{\mathrm{g}} / 4\right)$ WB \\
$\left(\rho_{\mathrm{g}} / 4\right) \mathrm{BB}$ \\
\end{tabular} & $\begin{array}{c}((1+e) / 2) W D \\
\left(\rho_{R} / 2\right) W R \\
\left(\rho_{B} / 2\right) W B\end{array}$ \\
\hline DR/XY & $\begin{array}{l}(1 / 4) \mathrm{DD} \\
(1 / 2) \mathrm{DR} \\
(1 / 4) \mathrm{RR} \\
\end{array}$ & $\begin{array}{l}(1 / 2) D R \\
(1 / 2) R R\end{array}$ & $\begin{array}{l}(1 / 4) D R \\
(1 / 4) D B \\
(1 / 4) R R \\
(1 / 4) R B\end{array}$ & $\begin{array}{l}\text { (1/4)WD } \\
(1 / 4) W R \\
(1 / 4) D R \\
(1 / 4) R R\end{array}$ & $\begin{array}{l}\text { (1/4)WD } \\
(1 / 4) W R \\
(1 / 4) D B \\
(1 / 4) R B\end{array}$ & $\begin{array}{l}(1 / 2) W D \\
(1 / 2) W R\end{array}$ \\
\hline$D B / X Y$ & $\begin{array}{l}1 / 4) \mathrm{DD} \\
(1 / 4) \mathrm{DR} \\
(1 / 4) \mathrm{DB} \\
(1 / 4) \mathrm{RB} \\
\end{array}$ & $\begin{array}{l}(1 / 2) D R \\
(1 / 2) B R\end{array}$ & $\begin{array}{l}1 / 4) D R \\
(1 / 4) D B \\
(1 / 4) R B \\
(1 / 4) B B \\
\end{array}$ & $\begin{array}{l}1 / 1 / 4) W D \\
(1 / 4) W B \\
(1 / 4) D R \\
(1 / 4) R B\end{array}$ & $\begin{array}{l}\text { (1/4)WD } \\
(1 / 4) W B \\
1 / 4) D B \\
(1 / 4) B B\end{array}$ & $\begin{array}{l}(1 / 2) W D \\
(1 / 2) W B \\
\end{array}$ \\
\hline $\mathrm{RR} / \mathrm{XY}$ & $\begin{array}{l}(1 / 2) D R \\
(1 / 2) R R\end{array}$ & (1) $R R$ & $\begin{array}{l}(1 / 2) R B \\
(1 / 2) R R\end{array}$ & $\begin{array}{l}(1 / 2) W R \\
(1 / 2) R R\end{array}$ & $\begin{array}{l}(1 / 2) W R \\
(1 / 2) R B\end{array}$ & (1) WR \\
\hline $\mathrm{RB} / \mathrm{XY}$ & $\begin{array}{l}1 / 4) \mathrm{DR} \\
(1 / 4) \mathrm{RR} \\
(1 / 4) \mathrm{DB} \\
(1 / 4) \mathrm{RB} \\
\end{array}$ & $\begin{array}{l}(1 / 2) R R \\
(1 / 2) R B \\
\end{array}$ & $\begin{array}{l}(1 / 4) R R \\
(1 / 2) R B \\
(1 / 4) B B \\
\end{array}$ & $\begin{array}{l}1 / 4) W R \\
(1 / 4) W B \\
(1 / 4) R R \\
(1 / 4) R B \\
\end{array}$ & $\begin{array}{l}1 / 4) W R \\
(1 / 4) W B \\
1 / 4) \mathrm{RB} \\
(1 / 4) \mathrm{BB} \\
\end{array}$ & $\begin{array}{l}(1 / 2) W R \\
(1 / 2) W B \\
\end{array}$ \\
\hline $\mathrm{BB} / \mathrm{XY}$ & $\begin{array}{l}(1 / 2) D B \\
(1 / 2) R B\end{array}$ & (1) RB & $\begin{array}{l}(1 / 2) R B \\
(1 / 2) B B\end{array}$ & $\begin{array}{l}(1 / 2) W B \\
(1 / 2) R B\end{array}$ & $\begin{array}{l}(1 / 2) W B \\
(1 / 2) B B\end{array}$ & (1) WB \\
\hline WR/XY & $\begin{array}{l}(1 / 4) W D \\
(1 / 4) W R \\
1 / 4) D R \\
(1 / 4) R R\end{array}$ & $\begin{array}{l}(1 / 2) W R \\
(1 / 2) R R\end{array}$ & $\begin{array}{l}(1 / 4) W R \\
(1 / 4) W B \\
(1 / 4) R R \\
(1 / 4) R B\end{array}$ & $\begin{array}{l}(1 / 4) W W \\
(1 / 2) W R \\
(1 / 4) R R\end{array}$ & $\begin{array}{l}(1 / 4) W W \\
(1 / 4) W R \\
(1 / 4) R B \\
(1 / 4) W B\end{array}$ & $\begin{array}{l}(1 / 2) W W \\
(1 / 2) W R\end{array}$ \\
\hline WB/XY & $\begin{array}{l}\text { (1/4)WD } \\
(1 / 4) \mathrm{WR} \\
(1 / 4) \mathrm{DB} \\
(1 / 4) \mathrm{RB} \\
\end{array}$ & $\begin{array}{l}(1 / 2) W R \\
(1 / 2) R B \\
\end{array}$ & $\begin{array}{l}(1 / 4) \mathrm{WR} \\
(1 / 4) \mathrm{WB} \\
(1 / 4) \mathrm{RB} \\
(1 / 4) \mathrm{BB} \\
\end{array}$ & $\begin{array}{l}(1 / 4) W W \\
(1 / 4) W R \\
(1 / 4) \mathrm{WB} \\
(1 / 4) \mathrm{RB} \\
\end{array}$ & $\begin{array}{l}(1 / 4) W W \\
(1 / 2) W B \\
(1 / 4) B B \\
\end{array}$ & $\begin{array}{l}(1 / 2) W W \\
(1 / 2) W B\end{array}$ \\
\hline$W W / X Y$ & $\begin{array}{l}(1 / 2) W D \\
(1 / 2) W R\end{array}$ & (1) WR & $\begin{array}{l}(1 / 2) W R \\
(1 / 2) W B\end{array}$ & $\begin{array}{l}(1 / 2) W R \\
(1 / 2) W W\end{array}$ & $\begin{array}{l}(1 / 2) W B \\
(1 / 2) W W\end{array}$ & (1) $W W$ \\
\hline DD/XX & $\begin{array}{l}(1 / 2) D R / X X \\
(1 / 2) D D / X X\end{array}$ & (1) $D R / X X$ & $\begin{array}{l}(1 / 2) D R / X X \\
(1 / 2) D B / X X\end{array}$ & $\begin{array}{l}(1 / 2) W D / X X \\
(1 / 2) D R / X X\end{array}$ & $\begin{array}{l}(1 / 2) W D / X X \\
(1 / 2) D B / X X\end{array}$ & (1) WD/XX \\
\hline WD/XX & $\begin{array}{l}((1+\mathrm{e}) / 4) \mathrm{DD} / \mathrm{XX} \\
\left(\left(1+\mathrm{e}+\rho_{\mathrm{R}}\right) / \mathrm{LR}\right) \\
\mathrm{DR} / \mathrm{XX} \\
\left(\rho_{\mathrm{\rho}} / 4\right) \mathrm{RR} / \mathrm{XX} \\
\left(\rho_{\rho^{\prime}} / 4\right) \mathrm{DB} / \mathrm{XX} \\
\left(\rho_{\mathrm{g}} / 4\right) \mathrm{RB} / \mathrm{XX}\end{array}$ & $\begin{array}{r}((1+e) / 2) D R / X X \\
\left(\rho_{R} / 2\right) R R / X X \\
\left(\rho_{B} / 2\right) R B / X X\end{array}$ & \begin{tabular}{|l|}
$((1+e) / 4) D R / X X$ \\
$((1+e) / 4) D B / X X$ \\
$\left(\rho_{R} / 4\right) R R / X X$ \\
$\left(\left(\rho_{R}+\rho_{B}\right) / 4\right)$ \\
\multicolumn{1}{c}{$R B / X X$} \\
$\left(\rho_{B} / 4\right) B B / X X$
\end{tabular} & $\begin{array}{l}((1+\mathrm{e}) / 4) W D / X X \\
((1+\mathrm{e}) / 4) \mathrm{DR} / X X \\
\left(\rho_{\mathrm{R}} / 4\right) W R / X X \\
\left(\rho_{\mathrm{g}} / 4\right) \mathrm{RR} / \mathrm{XX} \\
\left(\rho_{\mathrm{g}} / 4\right) W B / X X \\
\left(\rho_{\mathrm{g}} / 4\right) \mathrm{RB} / \mathrm{XX}\end{array}$ & \begin{tabular}{|l|}
$((1+\mathrm{e}) / 4) W D / X X$ \\
$((1+\mathrm{e}) / 4) D B / X X$ \\
$\left(\rho_{\mathrm{R}} / 4\right) W R / X X$ \\
$\left(\rho_{\mathrm{R}} / 4\right) \mathrm{RB} / X X$ \\
$\left(\rho_{\mathrm{g}} / 4\right) W B / X X$ \\
$\left(\rho_{\mathrm{g}} / 4\right) \mathrm{BB} / X X$
\end{tabular} & $\begin{array}{r}((1+e) / 2) W D / X X \\
\left(\rho_{R} / 2\right) W R / X X \\
\left(\rho_{8} / 2\right) W B / X X\end{array}$ \\
\hline $\mathrm{DB} / \mathrm{XX}$ & $\begin{array}{l}(1 / 4) \mathrm{DD} / \mathrm{XX} \\
(1 / 4) \mathrm{DR} / \mathrm{XX} \\
(1 / 4) \mathrm{DB} / \mathrm{XX} \\
(1 / 4) \mathrm{RB} / \mathrm{XX} \\
\end{array}$ & $\begin{array}{l}(1 / 2) D R / X X \\
(1 / 2) B R / X X \\
\end{array}$ & \begin{tabular}{|l|}
$(1 / 4) \mathrm{DR} / \mathrm{XX}$ \\
$(1 / 4) \mathrm{DB} / \mathrm{XX}$ \\
$(1 / 4) \mathrm{RB} / \mathrm{XX}$ \\
$(1 / 4) \mathrm{BB} / \mathrm{XX}$ \\
\end{tabular} & $\begin{array}{l}(1 / 4) W D / X X \\
(1 / 4) W B / X X \\
(1 / 4) D R / X X \\
(1 / 4) R B / X X \\
\end{array}$ & $\begin{array}{l}(1 / 4) W D / X X \\
(1 / 4) W B / X X \\
(1 / 4) D B / X X \\
(1 / 4) B B / X X \\
\end{array}$ & $\begin{array}{l}(1 / 2) W D / X X \\
(1 / 2) W B / X X \\
\end{array}$ \\
\hline $\mathrm{BB} / \mathrm{XX}$ & $\begin{array}{l}(1 / 2) D B / X X \\
(1 / 2) R B / X X\end{array}$ & (1) $R B / X X$ & $\begin{array}{l}(1 / 2) R B / X X \\
(1 / 2) B B / X X\end{array}$ & $\begin{array}{l}(1 / 2) W B / X X \\
(1 / 2) R B / X X\end{array}$ & $\begin{array}{l}(1 / 2) W B / X X \\
(1 / 2) B B / X X\end{array}$ & (1) $W B / X X$ \\
\hline
\end{tabular}

Fig. S10. Crosses representing the inheritance pattern of a CRISPR-based homing system targeting the tra locus in $C$. capitata. "D" denotes the drive allele, $\operatorname{tra}^{n C H E}$, "W" denotes the wild-type tra allele, "R" denotes a drive-resistant allele with an in-frame internal deletion at the tra locus, $\operatorname{tra}^{R s t}$ and " $\mathrm{B}$ " denotes a drive-resistant null allele tra. C. capitata is an $\mathrm{XY}$ species in which female development requires presence of the tra allele, hence XY individuals are fertile males, $\mathrm{XX}$ individuals with a functioning tra allele (i.e. having the genotypes WW, WR, WB, RR, DR and RB) are fertile females, and XX individuals without a functioning tra allele (i.e. having the genotypes DD, DB and BB) are fertile males. The only exception is WD/XX individuals, which are infertile intersex individuals, unless a uniform somatic destruction of the tra locus in all cells is guaranteed by using multiple guide RNAs and an early embryogenic promoter such as $S_{r y-} \alpha$ promoter. Homing is only manifest in WD heterozygotes, whereby WD individuals produce $\mathrm{D}$ gametes in the germline at a frequency equal to $(1+e) / 2$, where $e$ denotes the proportion of $\mathrm{W}$ gametes that are converted into $\mathrm{D}$ gametes through the act of homing. Homing-resistant alleles may be generated during the process of DNA cleavage and repair whereby WD individuals produce resistant alleles that are in-frame indels, $\mathrm{R}$, at a rate equal to $\rho_{\mathrm{R}} / 2$, and produce cleavage resistant alleles that are out-of-frame or other mutations, $\mathrm{B}$, at a rate equal to $\rho_{\mathrm{B}} / 2$. Crosses involving $\mathrm{WD} / \mathrm{XX}$ males are shaded out as $\mathrm{WD} / \mathrm{XX}$ individuals may be rendered either infertile intersex or fertile males as described above. Offspring are half XX and half XY. The inheritance pattern of the homing and resistant alleles depicted here is incorporated into the population dynamic model described above and in Fig. S11. 


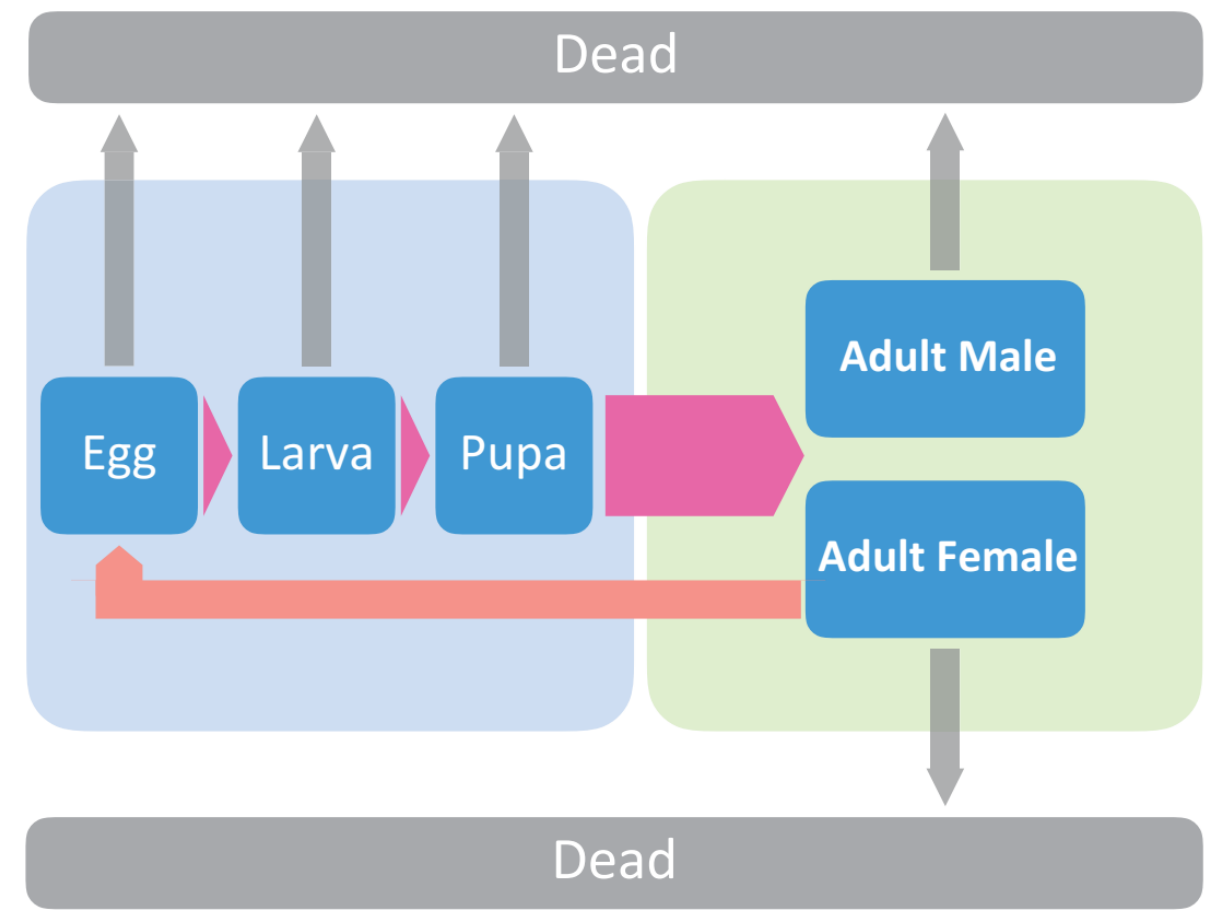

Fig. S11. Population dynamic model of $C$. capitata Eggs develop into larvae which develop into pupae, some of which develop into adult males and some into adult females. Death can occur at any life stage, and adult females lay eggs following fertilization. Additional density-dependent mortality occurs at the larval stage. Parameter values are provided in Table S2 and the equations describing the equivalent implementation of this model for An. gambiae are included in Supplementary File S1 of Marshall et al., 2017 (163). 
Table S1. Parameter values for population genetic/dynamic model for C. capitata.

\begin{tabular}{|c|c|c|c|}
\hline Symbol: & Parameter: & Value: & References: \\
\hline \multicolumn{4}{|c|}{ Primary parameters: } \\
\hline$\beta$ & Egg production per adult female & $20 /$ day & Diamantidis et al., 2011 \\
\hline$T_{E}$ & Duration of egg stage & 2 days & Diamantidis et al., 2011 \\
\hline$T_{L}$ & Duration of larval stage & 6 days & Diamantidis et al., 2011 \\
\hline$T_{P}$ & Duration of pupal stage & 10 days & Diamantidis et al., 2011 \\
\hline$M$ & Death rate of adult stage & $0.1 /$ day & Carey et al., 2005 (167) \\
\hline$r_{M}$ & $\begin{array}{l}\text { Population growth rate (in absence of density- } \\
\text { dependent mortality) }\end{array}$ & $1.031 /$ day & $\begin{array}{l}\text { Nyamukondiwa et al., } \\
1980 \text { (165) }\end{array}$ \\
\hline$c$ & $\begin{array}{l}\text { Probability of CRISPR-mediated cleavage in WD } \\
\text { heterozygote }\end{array}$ & 1.0 & $\begin{array}{l}\text { Champer et al., } 2017 \\
\text { (163) }\end{array}$ \\
\hline$P_{H D R}$ & $\begin{array}{l}\text { Probability of accurate homology-directed repair } \\
\text { given cleavage }\end{array}$ & 0.90 & This paper \\
\hline \multicolumn{4}{|c|}{ Variable parameters: } \\
\hline$\theta$ & $\begin{array}{l}\text { Proportion of resistant alleles that are in-frame } \\
\text { internal deletions }\end{array}$ & $\begin{array}{l}{[1 / 3,(1 / 3) \mathrm{x}} \\
\left.10^{-4}\right]\end{array}$ & This paper \\
\hline$N$ & $\begin{array}{l}\text { Equilibrium adult medfly population size (male and } \\
\text { female) }\end{array}$ & {$\left[10^{3}, 10^{6}\right]$} & $\begin{array}{l}\text { Diamantidis et al., } 2011 \\
(166)\end{array}$ \\
\hline
\end{tabular}


Table S2. List of primers used in this study

\begin{tabular}{|c|c|c|}
\hline ID & Name & Sequence \\
\hline MK024 & DTra_F & CGGCGACAAGCTTGAGGTACCCACTATATAGTATAAC \\
\hline MK025 & DTra_R & CTATAGGGCGAATTGTGTAGCCAAATCGCGGAACTC \\
\hline MK037 & G1-S & CTTCAGCAGTGGAACCCAGCATCG \\
\hline MK038 & G1-AS & AAACCGATGCTGGGTTCCACTGCT \\
\hline MK041 & G3-S & CTTCGATCGGTTATACTATATAGT \\
\hline MK042 & G3-AS & AAACACTATATAGTATAACCGATC \\
\hline MK043 & G4-S & CTTCGTGCTGGGTTCCACTGCTGT \\
\hline MK044 & G4-AS & AAACACAGCAGTGGAACCCAGCAC \\
\hline MK048 & G5-S & CTTCTATAGTGGGTACCTCGATGC \\
\hline MK049 & G5-AS & AAACGCATCGAGGTACCCACTATA \\
\hline MK058 & Tra_T7endo_F & CCTGCTAATTCTGCTTTCCCTATGTTTGTG \\
\hline MK059 & Tra_T7endo_R & CCTCGTCTGCAAAGTACGGAATCTTGTG \\
\hline MK060 & attB-SmaI-S & $\begin{array}{l}\text { CTAGCCGCGGTGCGGGTGCCAGGGCGTGCCCTTGGGCTCCCCGGGGAGCCCAA } \\
\text { GGGCACGCCCTGGCACCCGCACCGCGG }\end{array}$ \\
\hline MK061 & attB-SmaI-AS & $\begin{array}{l}\text { AGCTCCGCGGTGCGGGTGCCAGGGCGTGCCCTTGGGCTCCCCGGGGAGCCCAA } \\
\text { GGGCACGCCCTGGCACCCGCACCGCGG }\end{array}$ \\
\hline MK072 & aTubE1_F & CCAAGTGACCGCGGATCTTCATATTCGTTTTACGTTTGTCAAGCCTC \\
\hline MK073 & aTubE1_R & TCGTGGTCCTTATAGTCCATATTGAGTTTTTATTGGAAGTGTTTCAC \\
\hline MK075 & aTubI1GT_R & TCGTGGTCCTTATAGTCCTCAACCTGTGGATGAGGAGGAAGGGAAAACGGATG \\
\hline MK076 & Cas9DYK_F & GACTATAAGGACCACGACGGAGACTACAAGGATCATG \\
\hline MK077 & Cas9_R & GATCTAGATCTGCGGCCGATCACTAGATTACTTTTTCTTTTTTGCCTG \\
\hline MK078 & HMA_F1 & CGGTCACACTGAGGAAAGTG \\
\hline MK079 & HMA_R1.1 & CAACAAAAAGATGGCACTGG \\
\hline MK085 & Cas9_SeqR1 & TGGTGCTCGTCGTATCTC \\
\hline MK086 & Cas9_SeqR2 & TTGATAATTTTCAGCAGATCGTG \\
\hline MK087 & Cas9_SeqR3 & CTTGTTGTCGATGGAGTC \\
\hline MK088 & Cas9_SeqR4 & CAGCACAGAATAGGCCAC \\
\hline MK116 & 3xP3attP_F & $\begin{array}{l}\text { ACTGGGGTAACCTTTGAGTTCTCTCAGTTGGGGGCGTAGGGGGGATTATTCATT } \\
\text { AGAGAC }\end{array}$ \\
\hline MK117 & SV40attP_R & $\begin{array}{l}\text { GGGGTAACCTTTGAGTTCTCTCAGTTGGGGGCGTAGGGATGATGAGTTTGGACA } \\
\text { AACCAC }\end{array}$ \\
\hline MK122 & Transformer4_UR & CAAAGGTTACCCCAGTTGGGGCACTACTCTGTCGGCATCCATTTTCATC \\
\hline MK123 & Transformer4_DF & CTCAAAGGTTACCCCAGTTGGGGCACTACTGCAGTGGAACCCAGCATCGAG \\
\hline MK126 & Tra_HRCheck_F & CCGACCGAATCGTGAGGACTTGAAG \\
\hline MK127 & Tra_HRCheck_R & GAATTAAGTAACTTCCACTTCCTAACTCGTGTGAC \\
\hline MK128 & XFPct_F & AACGAGAAGCGCGATCACATGGTC \\
\hline MK129 & XFPnt_R & ACGCTGAACTTGTGGCCGTTTACGTC \\
\hline MK134 & attBaTubE1_F & $\begin{array}{l}\text { GGGCGCGTACTCCACCTCACCTAGGTGACTCGAGTTCATATTCGTTTTACGTTT } \\
\text { GTC }\end{array}$ \\
\hline MK135 & Cas_attBSmaI_AscIR & GGGCGCGTACTCCACCTCACGGCGCGCCATTACTTTTTCTTTTTTGCCTG \\
\hline MK138 & DPa180_AvrIIF & AATAACCTAGGTGGTGATCATTGTTCTTTCTTACTTGGTG \\
\hline MK139 & DPa180_XhoIR & TAATCCTCGAGTAATAATTTCCCCGTGTTGTGCTG \\
\hline MK140 & Rcd1r_XbaIF & AATAATCTAGACACGGCCAAATCGATGCAGAC \\
\hline MK141 & Rcd1r_XhoIR & TAATCCTCGAGGTTAGCTTGCAAAGATCTAGTAG \\
\hline MK142 & Srya_AvrIIF & AATAACCTAGGGCCACCAGCAGTTCAAGACCAAG \\
\hline MK143 & Srya_XhoIR & TAATCCTCGAGTATCAGATGTGCTCCGGGAAACAG \\
\hline MK144 & bTub3UTR_F & AAAAGTAATGGCGCGATTAACTTCCCACTCAAGATCAC \\
\hline MK145 & bTub3UTR_R & CGCTTAATGCGTATGGTTTAGGTTTATGCAATGCCT \\
\hline MK146 & U63P_F & CTGTTTTGCTCACCTGTGATTGCTCCTACTC \\
\hline MK147 & U63DS_R & CATACGCATTAAGCGAACATTAAAAAGATG \\
\hline MK148 & 3xP3attB_F & CCACCTCACGGCGCGGGGGATTATTCATTAGAGAC \\
\hline MK149 & SV40toU63_R & AGGTGAGCAAAACAGGATGATGAGTTTGGACAAACCAC \\
\hline MK153 & PhiC31_BsaI_F & ATGGTCTCACATGGACACGTACGCGGGTGCTTACGAC \\
\hline
\end{tabular}




\begin{tabular}{|l|l|l|}
\hline MK154 & PhiC31_NotI_R & GTGTATGCGGCCGCTTACTAGGCAGCTACGTCTTC \\
\hline MK155 & HRMA_OT1F & GGACCAGGAGCGTTATCTG \\
\hline MK156 & HRMA_OT1R & GGCAAATTGATGTCGAGCAC \\
\hline MK157 & HRMA_OT2F & CCATATCCGACCTGACCAC \\
\hline MK158 & HRMA_OT2R & CGGTTGCTGTTCCGTTTC \\
\hline MK159 & HRMA_OT3F & CAGCTTGTTGTCCTCGATG \\
\hline MK160 & HRMA_OT3R & GTGGCAGACCGAATCCAG \\
\hline
\end{tabular}




\section{SI References}

1. United Nations, Department of Economic and Social Affairs, Population Division. World population prospects Highlights, 2019 revision Highlights, 2019 revision. 2019.

2. Deutsch CA, Tewksbury JJ, Tigchelaar M, Battisti DS, Merrill SC, Huey RB, et al. Increase in crop losses to insect pests in a warming climate. Science. 2018 Aug 31;361(6405):916-9.

3. Global Issues: Food Security [Internet]. [cited 2019 Nov 17]. Available from: https://www.peacecorps.gov/educators/resources/global-issues-food-security/

4. Paini DR, Sheppard AW, Cook DC, De Barro PJ, Worner SP, Thomas MB. Global threat to agriculture from invasive species. Proc Natl Acad Sci USA. 2016 Jul 5;113(27):7575-9.

5. Sharma KR, Jaiswal DK, Babu SR, Saraoj AK. Impact of invasive insect pests species on agroecosystem in India and their management. :8.

6. Mutamiswa R, Chidawanyika F, Nyamukondiwa C. Dominance of spotted stemborer Chilo partellus Swinhoe (Lepidoptera: Crambidae) over indigenous stemborer species in Africa's changing climates: ecological and thermal biology perspectives. Agricultural and Forest Entomology. 2017;19(4):344-56.

7. Kumela T, Mendesil E, Enchalew B, Kassie M, Tefera T. Effect of the Push-Pull Cropping System on Maize Yield, Stem Borer Infestation and Farmers' Perception. Agronomy. 2019 Aug;9(8):452.

8. Yaninek JS, Herren HR. Introduction and spread of the cassava green mite, Mononychellus tanajoa (Bondar) (Acari: Tetranychidae), an exotic pest in Africa and the search for appropriate control methods: a review. Bulletin of Entomological Research. 1988 Mar;78(1):1-13.

9. Yaninek JS. Continental dispersal of the cassava green mite, an exotic pest in Africa, and implications for biological control. Exp Appl Acarol. 1988 Jun 1;4(3):211-24.

10. Shukla PT. Preliminary report on the green mite (Mononychellus tanajoa, Bonder) resistance in Tanzanian local cassava varieties. [Internet]. 1976 [cited 2019 Nov 17]. Available from: https://www.cabi.org/isc/abstract/19780558425

11. Westphal MI, Browne M, MacKinnon K, Noble I. The link between international trade and the global distribution of invasive alien species. Biol Invasions. 2008 Apr;10(4):391-8.

12. Hauser M. A historic account of the invasion of Drosophila suzukii (Matsumura) (Diptera: Drosophilidae) in the continental United States, with remarks on their identification. Pest Management Science. 2011;67(11):1352-7. 
13. Walsh DB, Bolda MP, Goodhue RE, Dreves AJ, Lee J, Bruck DJ, et al. Drosophila suzukii (Diptera: Drosophilidae): Invasive Pest of Ripening Soft Fruit Expanding its Geographic Range and Damage Potential. Journal of Integrated Pest Management. 2011 Apr 1;2(1):G1-7.

14. Mitsui H, Achterberg KV, Nordlander G, Kimura MT. Geographical distributions and host associations of larval parasitoids of frugivorous Drosophilidae in Japan. Journal of Natural History. 2007 Sep 1;41(25-28):1731-8.

15. Bolda MP, Goodhue RE, Zalom FG. Spotted Wing Drosophila: Potential Economic Impact of a Newly Established Pest. : 4.

16. Calabria G, Máca J, Bächli G, Serra L, Pascual M. First records of the potential pest species Drosophila suzukii (Diptera: Drosophilidae) in Europe. Journal of Applied Entomology. 2012;136(1-2):139-47.

17. Baroffio C, Fischer S. Neue Bedrohung für Obstplantagen und Beerenpflanzen: Die Kirschessigfliege. [Internet]. [cited 2019 Nov 17]. Available from: https://ira.agroscope.ch/de$\mathrm{CH} /$ publication/28097

18. EPPO Global Database [Internet]. [cited 2019 Nov 17]. Available from: https://gd.eppo.int/reporting/article-2416

19. Vogt H, Baufeld P, Gross J, Koppler K, Hoffmann C. Drosophila suzukii: eine neue Bedrohung für den Europäischen Obst- und Weinbau. Bericht über eine internationale Tagung in Trient, 2. Dezember 2011. Journal für Kulturpflanzen. 2012;64(2):68-72.

20. HARRIS A, SHAW B. First record of Drosophila suzukii (Matsumura) (Diptera, Drosophilidae) in Great Britain. Dipterists Digest. 2014;21:8.

21. Kiss B, Lengyel GD, Kárpáti Z. First record of spotted wing drosophila [Drosophila suzukii (Matsumura, 1931)] in Hungary. [Internet]. 2013 [cited 2019 Nov 17]. Available from: https://www.cabi.org/isc/abstract/20133128433

22. Lengyel GD, Orosz S, Kiss B, Lupták R, Kárpáti Z. New records and present status of the invasive Spotted Wing Drosophila, Drosophila suzukii (Matsumura, 1931) (Diptera) in Hungary. Acta Zool Acad Sci H. 2015;61(1):73-80.

23. Orhan A, Aslantaş R, Önder BŞ, Tozlu G. First record of the invasive vinegar fly Drosophila suzukii (Matsumura) (Diptera: Drosophilidae) from eastern Turkey. Turk J Zool. :4.

24. Deprá M, Poppe JL, Schmitz HJ, De Toni DC, Valente VLS. The first records of the invasive pest Drosophila suzukii in the South American continent. J Pest Sci. 2014 Sep;87(3):379-83. 
25. Lavagnino NJ, Díaz BM, Cichón LI, De la Vega GJ, Garrido SA, Lago JD, et al. New records of the invasive pest Drosophila suzukii (Matsumura) (Diptera: Drosophilidae) in the South American continent. Rev Soc Entomol Arg. 2018 Mar 30;77(1):27-31.

26. Parchami-Araghi M, Gilasian E, Keyhanian A. Spotted Wing Drosophila, Drosophila suzukii (Matsumura) (Dip.: Drosophilidae), an invasive fruit pest new to the Middle East and Iran. Drosophila Information Service. 2015 Jan 1;98:59-60.

27. Burrack HJ, Fernandez GE, Spivey T, Kraus DA. Variation in selection and utilization of host crops in the field and laboratory by Drosophila suzukii Matsumara (Diptera: Drosophilidae), an invasive frugivore. Pest Management Science. 2013;69(10):1173-80.

28. Hauser M, Gaimari S, Damus M. Drosophila suzukii new to North America. Fly Times. $2009 ;(43): 12-5$.

29. Walton V, Lee J, Bruck D, Shearer P, Parent E, Whitney T, et al. Recognizing Fruit Damaged by Spotted Wing Drosophila (SWD), Drosophila suzukii [Internet]. Oregon, USA: Oregon State University Extension Service; 2010 [cited 2019 Nov 17]. Report No.: EM 9021. Available from: https://catalog.extension.oregonstate.edu/sites/catalog/files/project/pdf/em9021.pdf

30. Lee JC, Bruck DJ, Curry H, Edwards D, Haviland DR, Van Steenwyk RA, et al. The susceptibility of small fruits and cherries to the spotted-wing drosophila, Drosophila suzukii. Pest Manag Sci. 2011 Nov;67(11):1358-67.

31. Lee JC, Dreves AJ, Cave AM, Kawai S, Isaacs R, Miller JC, et al. Infestation of Wild and Ornamental Noncrop Fruits by Drosophila suzukii (Diptera: Drosophilidae). Annals of the Entomological Society of America. 2015 Mar 1;108(2):117-29.

32. De Ros G, Conci S, Pantezzi T, Savini G. The economic impact of invasive pest Drosophila suzukii on berry production in\&nbsp;the\&nbsp;Province of Trento, Italy. Journal of Berry Research. 2015 Jan 1;5(2):89-96.

33. Goodhue RE, Bolda M, Farnsworth D, Williams JC, Zalom FG. Spotted wing drosophila infestation of California strawberries and raspberries: economic analysis of potential revenue losses and control costs. Pest Management Science. 2011;67(11):1396-402.

34. Fava ED, Ioriatti C, Melegaro A. Cost-benefit analysis of controlling the spotted wing drosophila (Drosophila suzukii (Matsumura)) spread and infestation of soft fruits in Trentino, Northern Italy. Pest Management Science. 2017;73(11):2318-27.

35. Bruck DJ, Bolda M, Tanigoshi L, Klick J, Kleiber J, DeFrancesco J, et al. Laboratory and field comparisons of insecticides to reduce infestation of Drosophila suzukii in berry crops. Pest Management Science. 2011;67(11):1375-85. 
36. Van Timmeren S, Isaacs R. Control of spotted wing drosophila, Drosophila suzukii, by specific insecticides and by conventional and organic crop protection programs. Crop Protection. 2013 Dec 1;54:126-33.

37. Cuthbertson AGS, Collins DA, Blackburn LF, Audsley N, Bell HA. Preliminary Screening of Potential Control Products against Drosophila suzukii. Insects. 2014 Jun;5(2):488-98.

38. Knipling EF. Possibilities of Insect Control or Eradication Through the Use of Sexually Sterile Males1. Journal of Economic Entomology. 1955 Aug 1;48(4):459-62.

39. Klassen W, Curtis CF. History of the Sterile Insect Technique. In: Dyck VA, Hendrichs J, Robinson AS, editors. Sterile Insect Technique: Principles and Practice in Area-Wide Integrated Pest Management [Internet]. Dordrecht: Springer Netherlands; 2005 [cited 2019 Nov 17]. p. 336. Available from: https://doi.org/10.1007/1-4020-4051-2_1

40. Klassen W. AREA-WIDE INTEGRATED PEST MANAGEMENT AND THE STERILE INSECT TECHNIQUE. In 2005.

41. Vargas-Terán M, Hofmann HC, Tweddle NE. Impact of Screwworm Eradication Programmes Using the Sterile Insect Technique. In: Dyck VA, Hendrichs J, Robinson AS, editors. Sterile Insect Technique [Internet]. Berlin/Heidelberg: Springer-Verlag; 2005 [cited 2019 Nov 17]. p. 629-50. Available from: http://link.springer.com/10.1007/1-4020-4051-2_24

42. Lindquist DA, Abusowa M, Hall MJR. The New World screwworm fly in Libya: a review of its introduction and eradication. Medical and Veterinary Entomology. 1992;6(1):2-8.

43. Lance D, Mcinnis DO. BIOLOGICAL BASIS OF THE STERILE INSECT TECHNIQUE. In 2005.

44. McInnis DO, Vargas RI. Field Evaluation of a Medfly Genetic Sexing Strain in Hawaii. In: Aluja M, Liedo P, editors. Fruit Flies. New York, NY: Springer; 1993. p. 95-102.

45. Franz G. Recombination between homologous autosomes in medfly (Ceratitis capitata) males: type- 1 recombination and the implications for the stability of genetic sexing strains. Genetica. 2002 Sep;116(1):73-84.

46. Kerremans P, Franz G. Cytogenetic analysis of chromosome 5 from the Mediterranean fruit fly, Ceratitis capitata. Chromosoma. 1994 Apr 1;103(2):142-6.

47. Franz G. Genetic Sexing Strains in Mediterranean Fruit Fly, an Example for Other Species Amenable to Large-Scale Rearing for the Sterile Insect Technique. In 2005.

48. Meza JS, Haq I ul, Vreysen MJB, Bourtzis K, Kyritsis GA, Cáceres C. Comparison of classical and transgenic genetic sexing strains of Mediterranean fruit fly (Diptera: Tephritidae) for application of the sterile insect technique. PLOS ONE. 2018 Dec 14;13(12):e0208880. 
49. Schetelig MF, Handler AM. A transgenic embryonic sexing system for Anastrepha suspensa (Diptera: Tephritidae). Insect Biochemistry and Molecular Biology. 2012 Oct;42(10):790-5.

50. Ogaugwu CE, Schetelig MF, Wimmer EA. Transgenic sexing system for Ceratitis capitata (Diptera: Tephritidae) based on female-specific embryonic lethality. Insect Biochemistry and Molecular Biology. 2013 Jan;43(1):1-8.

51. Yan Y, Scott MJ. A transgenic embryonic sexing system for the Australian sheep blow fly Lucilia cuprina. Sci Rep. 2015 Dec;5(1):16090.

52. Patterson RS, Lofgren CS, Boston MD. The Sterile-Male Technique for Control of Mosquitoes: A Field Cage Study with Anopheles quadrimaculatus. The Florida Entomologist. 1968;51(2):77-82.

53. Curtis CF, Grover KK, Suguna SG, Uppal DK, Dietz K, Agarwal HV, et al. Comparative field cage tests of the population suppressing efficiency of three genetic control systems for Aedes aegypti. Heredity. 1976 Feb;36(1):11-29.

54. Bakri A, Mehta K, Lance DR. Sterilizing Insects with Ionizing Radiation. In: Dyck VA, Hendrichs J, Robinson AS, editors. Sterile Insect Technique: Principles and Practice in AreaWide Integrated Pest Management [Internet]. Dordrecht: Springer Netherlands; 2005 [cited 2019 Nov 17]. p. 233-68. Available from: https://doi.org/10.1007/1-4020-4051-2_9

55. Horn C, Wimmer EA. A transgene-based, embryo-specific lethality system for insect pest management. Nat Biotechnol. 2003 Jan;21(1):64-70.

56. Schetelig MF, Caceres C, Zacharopoulou A, Franz G, Wimmer EA. Conditional embryonic lethality to improve the sterile insect technique in Ceratitis capitate (Diptera: Tephritidae). BMC Biol. 2009 Dec;7(1):4.

57. Gossen M, Bujard H. Tight control of gene expression in mammalian cells by tetracyclineresponsive promoters. Proceedings of the National Academy of Sciences. 1992 Jun 15;89(12):5547-51.

58. Urlinger S, Baron U, Thellmann M, Hasan MT, Bujard H, Hillen W. Exploring the sequence space for tetracycline-dependent transcriptional activators: Novel mutations yield expanded range and sensitivity. Proceedings of the National Academy of Sciences. 2000 Jul 5;97(14):79638 .

59. Thomas DD, Donnelly CA, Wood RJ, Alphey LS. Insect Population Control Using a Dominant, Repressible, Lethal Genetic System. Science. 2000 Mar 31;287(5462):2474-6.

60. Catteruccia F, Benton JP, Crisanti A. An Anopheles transgenic sexing strain for vector control. Nat Biotechnol. 2005 Nov;23(11):1414-7. 
61. Scolari F, Schetelig MF, Bertin S, Malacrida AR, Gasperi G, Wimmer EA. Fluorescent sperm marking to improve the fight against the pest insect Ceratitis capitata (Wiedemann; Diptera: Tephritidae). New Biotechnology. 2008 Jun;25(1):76-84.

62. Zimowska GJ, Nirmala X, Handler AM. The $\beta 2$-tubulin gene from three tephritid fruit fly species and use of its promoter for sperm marking. Insect Biochemistry and Molecular Biology. 2009 Aug;39(8):508-15.

63. Ahmed HMM, Hildebrand L, Wimmer EA. Improvement and Use of CRISPR/Cas9 to Engineer a Sperm-marking Strain for the Invasive Fruit Pest Drosophila suzukii. 2019 Aug 14 [cited 2019 Nov 2]; Available from: https://www.researchsquare.com/article/804bbff3-022844d5-8e09-3acb0d8e408e/v1

64. Schetelig MF, Wimmer EA. Insect Transgenesis and the Sterile Insect Technique. In: Vilcinskas A, editor. Insect Biotechnology [Internet]. Dordrecht: Springer Netherlands; 2011 [cited 2019 Nov 3]. p. 169-94. (Biologically-Inspired Systems). Available from: https://doi.org/10.1007/978-90-481-9641-8_9

65. Berghammer AJ, Klingler M, Wimmer EA. A universal marker for transgenic insects. Nature. 1999 Nov;402(6760):370-1.

66. Spradling AC, Rubin GM. Transposition of cloned P elements into Drosophila germ line chromosomes. Science. 1982 Oct 22;218(4570):341-7.

67. Hacker U, Nystedt S, Barmchi MP, Horn C, Wimmer EA. piggyBac-based insertional mutagenesis in the presence of stably integrated P elements in Drosophila. Proceedings of the National Academy of Sciences. 2003 Jun 24;100(13):7720-5.

68. Klemenz R, Weber U, Gehring WJ. The white gene as a marker in a new P-element vector for gene transfer in Drosophila. Nucl Acids Res. 1987;15(10):3947-59.

69. Handler AM, Ii RAH. Germline transformation of Drosophila melanogaster with the piggyBac transposon vector. Insect Molecular Biology. 1999;9.

70. Masumoto M, Ohde T, Shiomi K, Yaginuma T, Niimi T. A Baculovirus Immediate-Early Gene, ie1, Promoter Drives Efficient Expression of a Transgene in Both Drosophila melanogaster and Bombyx mori. Singh A, editor. PLoS ONE. 2012 Nov 13;7(11):e49323.

71. Li J, Handler AM. Temperature-dependent sex-reversal by a transformer-2 gene-edited mutation in the spotted wing drosophila, Drosophila suzukii. Sci Rep. 2017 Dec;7(1):12363.

72. Horn C, Wimmer EA. A versatile vector set for animal transgenesis. Development genes and evolution. 2000 Dec;210(12):630-7. 
73. Caroti F, Urbansky S, Wosch M, Lemke S. Germ line transformation and in vivo labeling of nuclei in Diptera: report on Megaselia abdita (Phoridae) and Chironomus riparius (Chironomidae). Dev Genes Evol. 2015 Jun;225(3):179-86.

74. Gonzalez-Estevez C, Momose T, Gehring WJ, Salo E. Transgenic planarian lines obtained by electroporation using transposon-derived vectors and an eye-specific GFP marker. Proceedings of the National Academy of Sciences. 2003 Nov 25;100(24):14046-51.

75. Holtzman S, Miller D, Eisman RC, Kuwayama H, Niimi T, Kaufman TC. Transgenictools for members of the genus Drosophila with sequenced genomes. Fly. 2010 Oct;4(4):349-62.

76. McClintock B. The origin and behavior of mutable loci in maize. PNAS. 1950 Jun 1;36(6):34455.

77. Munoz-Lopez M, Garcia-Perez J. DNA Transposons: Nature and Applications in Genomics. CG. 2010 Apr 1;11(2):115-28.

78. Rio DC. Identification and purification of a Drosophila protein that binds to the terminal 31base-pair inverted repeats of the P transposable element. Proc Natl Acad Sci USA. 1988;5.

79. Trubitsyna M, Michlewski G, Finnegan DJ, Elfick A, Rosser SJ, Richardson JM, et al. Use of mariner transposases for one-step delivery and integration of DNA in prokaryotes and eukaryotes by transfection. Nucleic Acids Research. 2017 Jun 2;45(10):e89-e89.

80. Lidholm DA, Lohe AR, Hartl DL. The transposable element mariner mediates germline transformation in Drosophila melanogaster. Genetics. 1993 Jul;134(3):859-68.

81. Medhora MM, MacPeek AH, Hartl DL. Excision of the Drosophila transposable element mariner: identification and characterization of the Mos factor. EMBO J. 1988 Jul;7(7):2185-9.

82. Cary LC, Goebel M, Corsaro BG, Wang HG, Rosen E, Fraser MJ. Transposon mutagenesis of baculoviruses: analysis of Trichoplusia ni transposon IFP2 insertions within the FP-locus of nuclear polyhedrosis viruses. Virology. 1989 Sep;172(1):156-69.

83. Yusa K, Zhou L, Li MA, Bradley A, Craig NL. A hyperactive piggyBac transposase for mammalian applications. Proceedings of the National Academy of Sciences. 2011 Jan $25 ; 108(4): 1531-6$.

84. Wimmer EA. Insect transgenesis by site-specific recombination. Nat Methods. 2005 Aug;2(8):580-2.

85. Siegal ML, Hartl DL. Transgene Coplacement and high efficiency site-specific recombination with the Cre/loxP system in Drosophila. Genetics. 1996 Oct;144(2):715-26. 
86. Long D-P, Zhao A-C, Chen X-J, Zhang Y, Lu W-J, Guo Q, et al. FLP RecombinaseMediated Site-Specific Recombination in Silkworm, Bombyx mori. Liu Z, editor. PLoS ONE. 2012 Jun 29;7(6):e40150.

87. Sauer B. Cre/Iox: One more step in the taming of the genome. Endocr. 2002 Dec 1;19(3):2217.

88. Zheng B, Sage M, Sheppeard EA, Jurecic V, Bradley A. Engineering Mouse Chromosomes with Cre-loxP: Range, Efficiency, and Somatic Applications. Molecular and Cellular Biology. 2000 Jan 15;20(2):648-55.

89. Oberdoerffer P. Unidirectional Cre-mediated genetic inversion in mice using the mutant loxP pair lox66/lox71. Nucleic Acids Research. 2003 Nov 15;31(22):140e-140.

90. Medberry SL, Dale E, Qin M, Ow DW. Intra-chromosomal rearrangements generated by Crelox site-specific recombination. Nucl Acids Res. 1995;23(3):485-90.

91. Horn C, Handler AM. Site-specific genomic targeting in Drosophila. Proceedings of the National Academy of Sciences. 2005 Aug 30;102(35):12483-8.

92. Siegal ML, Hartl DL. Application of Cre/loxP in Drosophila. In: Tuan RS, Lo CW, editors. Developmental Biology Protocols: Volume II [Internet]. Totowa, NJ: Humana Press; 2000 [cited 2019 Nov 17]. p. 487-95. (Methods in Molecular Biology ${ }^{\mathrm{TM}}$ ). Available from: https://doi.org/10.1385/1-59259-065-9:487

93. Baer A, Bode J. Coping with kinetic and thermodynamic barriers: RMCE, an efficient strategy for the targeted integration of transgenes. Current Opinion in Biotechnology. 2001 Oct 1;12(5):473-80.

94. Rausch H, Lehmann M. Structural analysis of the actinophae Acids Res. 1991 Oct 11;19(19):5187-9.

95. Rutherford K, Yuan P, Perry K, Sharp R, Van Duyne GD. Attachment site recognition and regulation of directionality by the serine integrases. Nucleic Acids Res. 2013 Sep;41(17):834156.

96. Thorpe HM, Wilson SE, Smith MCM. Control of directionality in the site-specific recombination system of the Streptomyces phage $\varphi$ C31. Molecular Microbiology. 2000;38(2):232-41.

97. Bateman JR, Lee AM, Wu C -ting. Site-Specific Transformation of Drosophila via $\phi C 31$ Integrase-Mediated Cassette Exchange. Genetics. 2006 Jun;173(2):769-77.

98. Driever W, Solnica-Krezel L, Schier AF, Neuhauss SC, Malicki J, Stemple DL, et al. A genetic screen for mutations affecting embryogenesis in zebrafish. Development. 1996 Dec;123:37-46. 
99. Justice MJ. Capitalizing on large-scale mouse mutagenesis screens. Nat Rev Genet. 2000 Nov;1(2):109-15.

100. Favor J, Neuhäuser-Klaus A. Saturation mutagenesis for dominant eye morphological defects in the mouse Mus musculus. Mamm Genome. 2000 Jul;11(7):520-5.

101. Nadeau JH, Frankel WN. The roads from phenotypic variation to gene discovery: mutagenesis versus QTLs. Nat Genet. 2000 Aug;25(4):381-4.

102. Lanzov VA. Gene Targeting for Gene Therapy: Prospects. Molecular Genetics and Metabolism. 1999 Oct 1;68(2):276-82.

103. Bibikova M, Carroll D, Segal DJ, Trautman JK, Smith J, Kim Y-G, et al. Stimulation of Homologous Recombination through Targeted Cleavage by Chimeric Nucleases. Molecular and Cellular Biology. 2001 Jan 1;21(1):289-97.

104. Bibikova M, Beumer K, Trautman JK, Carroll D. Enhancing gene targeting with designed zinc finger nucleases. Science. 2003 May 2;300(5620):764.

105. Bibikova M, Golic M, Golic KG, Carroll D. Targeted Chromosomal Cleavage and Mutagenesis in Drosophila Using Zinc-Finger Nucleases. Genetics. 2002 Jul 1;161(3):1169-75.

106. Liang F, Han M, Romanienko PJ, Jasin M. Homology-directed repair is a major double-strand break repair pathway in mammalian cells. PNAS. 1998 Apr 28;95(9):5172-7.

107. Choulika A, Perrin A, Dujon B, Nicolas JF. Induction of homologous recombination in mammalian chromosomes by using the I-SceI system of Saccharomyces cerevisiae. Mol Cell Biol. 1995 Apr;15(4):1968-73.

108. Kim YG, Cha J, Chandrasegaran S. Hybrid restriction enzymes: zinc finger fusions to Fok I cleavage domain. Proceedings of the National Academy of Sciences. 1996 Feb 6;93(3):1156-60.

109. Miller JC, Tan S, Qiao G, Barlow KA, Wang J, Xia DF, et al. A TALE nuclease architecture for efficient genome editing. Nat Biotechnol. 2011 Feb;29(2):143-8.

110. Reyon D, Tsai SQ, Khayter C, Foden JA, Sander JD, Joung JK. FLASH assembly of TALENs for high-throughput genome editing. Nat Biotechnol. 2012 May;30(5):460-5.

111. Barrangou R, Fremaux C, Deveau H, Richards M, Boyaval P, Moineau S, et al. CRISPR Provides Acquired Resistance Against Viruses in Prokaryotes. Science. 2007 Mar 23;315(5819):1709-12.

112. Makarova KS, Grishin NV, Shabalina SA, Wolf YI, Koonin EV. A putative RNA-interferencebased immune system in prokaryotes: computational analysis of the predicted enzymatic 
machinery, functional analogies with eukaryotic RNAi, and hypothetical mechanisms of action. Biology Direct. 2006 Mar 16;1(1):7.

113. Hsu PD, Lander ES, Zhang F. Development and Applications of CRISPR-Cas9 for Genome Engineering. Cell. 2014 Jun;157(6):1262-78.

114. Cong L, Ran FA, Cox D, Lin S, Barretto R, Habib N, et al. Multiplex Genome Engineering Using CRISPR/Cas Systems. Science. 2013 Feb 15;339(6121):819-23.

115. Bassett AR, Tibbit C, Ponting CP, Liu J-L. Highly Efficient Targeted Mutagenesis of Drosophila with the CRISPR/Cas9 System. Cell Rep. 2013 Jul 11;4(1):220-8.

116. Anders C, Niewoehner O, Duerst A, Jinek M. Structural basis of PAM-dependent target DNA recognition by the Cas9 endonuclease. Nature. 2014 Sep;513(7519):569-73.

117. Hall B, Cho A, Limaye A, Cho K, Khillan J, Kulkarni AB. Genome Editing in Mice Using CRISPR/Cas9 Technology. Current Protocols in Cell Biology. 2018;81(1):e57.

118. Hwang WY, Fu Y, Reyon D, Maeder ML, Tsai SQ, Sander JD, et al. Efficient genome editing in zebrafish using a CRISPR-Cas system. Nat Biotechnol. 2013 Mar;31(3):227-9.

119. Sternberg SH, Redding S, Jinek M, Greene EC, Doudna JA. DNA interrogation by the CRISPR RNA-guided endonuclease Cas9. Nature. 2014 Mar;507(7490):62-7.

120. Lin S, Ewen-Campen B, Ni X, Housden BE, Perrimon N. In Vivo Transcriptional Activation Using CRISPR/Cas9 in Drosophila. Genetics. 2015 Oct 1;201(2):433-42.

121. La Russa MF, Qi LS. The New State of the Art: Cas9 for Gene Activation and Repression. Mol Cell Biol. 2015 Nov 15;35(22):3800-9.

122. Mali P, Aach J, Stranges PB, Esvelt KM, Moosburner M, Kosuri S, et al. CAS9 transcriptional activators for target specificity screening and paired nickases for cooperative genome engineering. Nat Biotechnol. 2013 Sep;31(9):833-8.

123. Gilbert LA, Horlbeck MA, Adamson B, Villalta JE, Chen Y, Whitehead EH, et al. GenomeScale CRISPR-Mediated Control of Gene Repression and Activation. Cell. 2014 Oct 23;159(3):647-61.

124. Xie N, Zhou Y, Sun Q, Tang B. Novel Epigenetic Techniques Provided by the CRISPR/Cas9 System. Stem Cells Int [Internet]. 2018 Jul 8 [cited 2019 Nov 17];2018. Available from: https://www.ncbi.nlm.nih.gov/pmc/articles/PMC6079388/

125. Kang JG, Park JS, Ko J-H, Kim Y-S. Regulation of gene expression by altered promoter methylation using a CRISPR/Cas9-mediated epigenetic editing system. Sci Rep. 2019 Aug 19;9(1):1-12. 
126. Pulecio J, Verma N, Mejía-Ramírez E, Huangfu D, Raya A. CRISPR/Cas9-Based Engineering of the Epigenome. Cell Stem Cell. 2017 Oct;21(4):431-47.

127. Satomura A, Nishioka R, Mori H, Sato K, Kuroda K, Ueda M. Precise genome-wide base editing by the CRISPR Nickase system in yeast. Sci Rep. 2017 May 18;7(1):1-10.

128. Gopalappa R, Suresh B, Ramakrishna S, Kim H (Henry). Paired D10A Cas9 nickases are sometimes more efficient than individual nucleases for gene disruption. Nucleic Acids Research. 2018 Jul 6;46(12):e71-e71.

129. Trevino AE, Zhang F. Chapter Eight - Genome Editing Using Cas9 Nickases. In: Doudna JA, Sontheimer EJ, editors. Methods in Enzymology [Internet]. Academic Press; 2014 [cited 2019 Nov 17]. p. 161-74. (The Use of CRISPR/Cas9, ZFNs, and TALENs in Generating SiteSpecific Genome Alterations; vol. 546). Available from: http://www.sciencedirect.com/science/article/pii/B9780128011850000088

130. Kim YB, Komor AC, Levy JM, Packer MS, Zhao KT, Liu DR. Increasing the genometargeting scope and precision of base editing with engineered Cas9-cytidine deaminase fusions. Nat Biotechnol. 2017 Apr;35(4):371-6.

131. Zong Y, Wang Y, Li C, Zhang R, Chen K, Ran Y, et al. Precise base editing in rice, wheat and maize with a Cas9-cytidine deaminase fusion. Nat Biotechnol. 2017 May;35(5):438-40.

132. Kim K, Ryu S-M, Kim S-T, Baek G, Kim D, Lim K, et al. Highly efficient RNA-guided base editing in mouse embryos. Nat Biotechnol. 2017 May;35(5):435-7.

133. Cheng T-L, Li S, Yuan B, Wang X, Zhou W, Qiu Z. Expanding C-T base editing toolkit with diversified cytidine deaminases. Nat Commun. 2019 Dec;10(1):3612.

134. Tan J, Zhang F, Karcher D, Bock R. Engineering of high-precision base editors for site-specific single nucleotide replacement. Nat Commun. 2019 Jan 25;10(1):1-10.

135. Zetsche B, Gootenberg JS, Abudayyeh OO, Slaymaker IM, Makarova KS, Essletzbichler P, et al. Cpf1 Is a Single RNA-Guided Endonuclease of a Class 2 CRISPR-Cas System. Cell. 2015 Oct;163(3):759-71.

136. Gantz VM, Jasinskiene N, Tatarenkova O, Fazekas A, Macias VM, Bier E, et al. Highly efficient Cas9-mediated gene drive for population modification of the malaria vector mosquito Anopheles stephensi. Proc Natl Acad Sci USA. 2015 Dec 8;112(49):E6736-43.

137. Hammond A, Galizi R, Kyrou K, Simoni A, Siniscalchi C, Katsanos D, et al. A CRISPR-Cas9 gene drive system targeting female reproduction in the malaria mosquito vector Anopheles gambiae. Nat Biotechnol. 2016 Jan;34(1):78-83. 
138. Gantz VM, Bier E. The mutagenic chain reaction: A method for converting heterozygous to homozygous mutations. Science. 2015 Apr 24;348(6233):442-4.

139. Galizi R, Hammond A, Kyrou K, Taxiarchi C, Bernardini F, O'Loughlin SM, et al. A CRISPR-Cas9 sex-ratio distortion system for genetic control. Sci Rep. 2016 Nov;6(1):31139.

140. Werren JH, Nur U, Wu C-I. Selfish genetic elements. Trends in Ecology \& Evolution. 1988 Nov 1;3(11):297-302.

141. Hurst GDD, Werren JH. The role of selfish genetic elements in eukaryotic evolution. Nat Rev Genet. 2001 Aug;2(8):597-606.

142. Chevalier BS. Homing endonucleases: structural and functional insight into the catalysts of intron/intein mobility. Nucleic Acids Research. 2001 Sep 15;29(18):3757-74.

143. Burt A. Site-specific selfish genes as tools for the control and genetic engineering of natural populations. Proc Biol Sci. 2003 May 7;270(1518):921-8.

144. Chatterjee P, Jakimo N, Jacobson JM. Minimal PAM specificity of a highly similar SpCas9 ortholog. Science Advances. 2018 Oct 1;4(10):eaau0766.

145. Kleinstiver BP, Prew MS, Tsai SQ, Topkar VV, Nguyen NT, Zheng Z, et al. Engineered CRISPR-Cas9 nucleases with altered PAM specificities. Nature. 2015 Jul;523(7561):481-5.

146. Kleinstiver BP, Prew MS, Topkar VV, Tsai SQ, Joung JK. 58. Engineered Cas9 Variants with Novel PAM Specificities Expand the Targeting Range of CRISPR/Cas Nucleases. Molecular Therapy. 2015 May 1;23:S26.

147. Unckless RL, Clark AG, Messer PW. Evolution of Resistance Against CRISPR/Cas9 Gene Drive. Genetics. 2016 Dec 10;

148. Port F, Chen H-M, Lee T, Bullock SL. Optimized CRISPR/Cas tools for efficient germline and somatic genome engineering in Drosophila. Proceedings of the National Academy of Sciences of the United States of America. 2014 Jul;111(29):E2967-76.

149. Protocols: Testing gRNA cleavage efficiency [Internet]. 2013. Available from: http://flycrispr.molbio.wisc.edu/protocols/embryonicPCR

150. Bassett AR, Tibbit C, Ponting CP, Liu J-L. Highly Efficient Targeted Mutagenesis of Drosophila with the CRISPR/Cas9 System. Cell Reports. 2013 Jul 11;4(1):220-8.

151. Zhang F. CRISPR DESIGN [Internet]. 2014. Available from: http://crispr.mit.edu

152. Groth AC, Olivares EC, Thyagarajan B, Calos MP. A phage integrase directs efficient sitespecific integration in human cells. Proceedings of the National Academy of Sciences of the United States of America. 2000 May;97(11):5995-6000. 
153. gRNA cloning protocols [Internet]. 2013. Available from: http://www.crisprflydesign.org/grnaexpression-vectors/

154. Schetelig MF, Handler AM. Strategy for enhanced transgenic strain development for embryonic conditional lethality in Anastrepha suspensa. Proceedings of the National Academy of Sciences of the United States of America. 2012 Jun;109(24):9348-53.

155. Chan YS, Huen DS, Glauert R, Whiteway E, Russell S. Optimising Homing Endonuclease Gene Drive Performance in a Semi-Refractory Species: The Drosophila melanogaster Experience. PLoS ONE. 2013;8(1).

156. Horn C, Schmid BGM, Pogoda FS, Wimmer EA. Fluorescent transformation markers for insect transgenesis. Insect biochemistry and molecular biology. 2002 Oct;32(10):1221-35.

157. Ibnsouda S, Schweisguth F, Jullien D, Kucherer C, Lepesant JA, Vincent A. Evolutionarily conserved positive and negative cis-acting elements control the blastoderm-specific expression of the Drosophila serendipity alpha cellularisation gene. Mechanisms of development. 1995 Jan;49(1-2):71-82.

158. Hirose F, Yamaguchi M, Handa H, Inomata Y, Matsukage A. Novel 8-base pair sequence (Drosophila DNA replication-related element) and specific binding factor involved in the expression of Drosophila genes for DNA polymerase alpha and proliferating cell nuclear antigen. The Journal of biological chemistry. 1993 Jan;268(3):2092-9.

159. Bischof J, Maeda RK, Hediger M, Karch F, Basler K. An optimized transgenesis system for Drosophila using germ-line-specific phiC31 integrases. Proceedings of the National Academy of Sciences of the United States of America. 2007;104:3312-7.

160. Ramos DM, Kamal F, Wimmer EA, Cartwright AN, Monteiro A. Temporal and spatial control of transgene expression using laser induction of the hsp70 promoter. BMC developmental biology. 2006 Nov;6:55.

161. Bateman JR, Lee AM, Wu CT. Site-specific transformation of Drosophila via phiC31 integrasemediated cassette exchange. Genetics. 2006;173:769-77.

162. Akbari OS, Bellen HJ, Bier E, Bullock SL, Burt A, Church GM, et al. Safeguarding gene drive experiments in the laboratory. Science. 2015 Aug 27;349(6251):927-9.

163. Marshall JM, Buchman A, Sánchez C. HM, Akbari OS. Overcoming evolved resistance to population-suppressing homing-based gene drives. Scientific Reports. 2017;7(1):3776.

164. Champer J, Reeves R, Oh SY, Liu C, Liu J, Clark AG, et al. Novel CRISPR/Cas9 gene drive constructs reveal insights into mechanisms of resistance allele formation and drive efficiency in genetically diverse populations. PLOS Genetics. 2017 Jul 20;13(7):e1006796. 
165. Nyamukondiwa C, Weldon CW, Chown SL, le Roux PC, Terblanche JS. Thermal biology, population fluctuations and implications of temperature extremes for the management of two globally significant insect pests. Journal of insect physiology. 2013 Dec;59(12):1199-211.

166. Diamantidis AD, Carey JR, Nakas CT, Papadopoulos NT. Population-specific demography and invasion potential in medfly. Ecology and evolution. 2011 Dec;1(4):479-88.

167. Carey JR, Liedo P, Vaupel JW. Mortality dynamics of density in the mediterranean fruit fly. Experimental Gerontology. 1995;30(6):605-29. 


\subsection{Hyperactive piggyBac transposase improves transformation efficiency in diverse insect species}

The use of transposon-based transformation vectors remains to be the first strategy to try when starting transgenesis work in a new insect organism. The versatile piggyBac transformation is widely used in many biological systems. This chapter presents tremendous improvement in piggyBac germline transformation in three different insect species from two different orders by using a mutated hyperactive piggyBactransposase. The important contribution of this work is correcting the previous perception that the hyperactive piggyBac transposase does not improve the efficiency of germline transformation. This is particularly relevant to those who are trying to develop biotechnological pest control strategies based on transgenesis.

Kolja N. Eckermann ${ }^{\Delta}$, Hassan M. M. Ahmed ${ }^{\Delta}$, Mohammad KaramiNejadRanjbar ${ }^{\Delta}$, Stefan Dippel, Christian E. Ogaugwu, Peter Kitzmann, Musa D. Isah \& Ernst A. Wimmer

$\Delta=$ Co-first authors

Authors contributions as stated in the published paper:

E.A.W., K.N.E., and S.D. conceived and designed the research; S.D., K.N.E., and M.K. designed the helper plasmids; K.N.E., M.K., P.K. and C.E.O. constructed the plasmids; K.N.E., M.K., and H.M.M.A. designed the experiments and performed the injections; K.N.E, M.K., H.M.M.A., and M.D.I performed the crossings and screenings; K.N.E., S.D., and H.M.M.A. performed the inverse PCR and K.N.E. analyzed the sequence data; K.N.E, S.D., and E.A.W. wrote the manuscript; K.N.E prepared the figures and tables; All authors edited and approved the final version of the manuscript.

My specific contributions were particularly to Table 1 and Fig. 1 with a minor contribution to Table 4.

Status of manuscript: Published in Insect Biochemistry and Molecular Biology (IBMB) in 2018.

DOI: $10.1016 / j . i b m b .2018 .04 .001$ 


\title{
Hyperactive piggyBac transposase improves transformation efficiency in diverse insect species
}

Kolja N. Eckermann ${ }^{\mathrm{a}, \mathrm{b}, \mathrm{c}, \dagger}$, Hassan M. M. Ahmed ${ }^{\mathrm{a}, \dagger}$, Mohammad KaramiNejadRanjbar ${ }^{\mathrm{a}, \dagger, 1}$, Stefan Dippel ${ }^{\mathrm{a}, \dagger, 2}$, Christian E. Ogaugwu ${ }^{\mathrm{a}, 3}$, Peter Kitzmann ${ }^{\mathrm{b}, \mathrm{d}}$, Musa D. Isah ${ }^{\mathrm{a}}$ \& Ernst A. Wimmer ${ }^{\mathrm{a}, \mathrm{b}, *}$

a Department of Developmental Biology, Johann-Friedrich-Blumenbach-Institute of Zoology and Anthropology, Göttingen Center of Molecular Biosciences, University of Göttingen, 37077 Göttingen, Germany. ${ }^{\mathrm{b}}$ Göttingen Graduate Center for Neurosciences, Biophysics, and Molecular Biosciences, University of Göttingen, 37077 Goettingen, Germany. ${ }^{c}$ Molecular Cell Dynamics, Max-Planck-Institute for Biophysical Chemistry, 37077 Göttingen, Germany. ${ }^{\mathrm{d} D e p a r t m e n t ~ o f ~ E v o l u t i o n a r y ~}$ Developmental Genetics, Johann-Friedrich-Blumenbach-Institute of Zoology and Anthropology, Göttingen Center of Molecular Biosciences, University of Göttingen, 37077 Göttingen, Germany.

*Corresponding Author: E. A. Wimmer. Tel +49 5513922888.

E-Mail address: ewimmer@gwdg.de (E.A. Wimmer).

\footnotetext{
† These authors contributed equally to this work.

${ }^{1}$ Current address: Weatherall Institute of Molecular Medicine, University of Oxford, Oxford OX3 9DU, UK.

${ }^{2}$ Current address: Department of Biology - Neurobiology/Ethology, Philipps-University Marburg, 35032 Marburg, Germany

${ }^{3}$ Current address: Department of Microbiology \& Molecular Genetics, University of California, Irvine, CA 92697.
}

\begin{abstract}
Even in times of advanced site-specific genome editing tools, the improvement of DNA transposases is still on high demand in the field of transgenesis: especially in emerging model systems where evaluated integrase landing sites have not yet been created and more importantly in non-model organisms such as agricultural pests and disease vectors, in which reliable sequence information and genome annotations are still pending. In fact, random insertional mutagenesis is essential to identify new genomic locations that are not influenced by position effects and thus can serve as future stable transgene integration sites. In this respect, a hyperactive version of the most widely used piggyBac transposase (PBase) has been engineered. The hyperactive version (hyPBase) is currently available with the original insect codon-based coding sequence ( $\left.{ }^{i} h y P B a s e\right)$ as well as in a mammalian codon-optimized ( ${ }^{m}$ hyPBase) version. Both facilitate significantly higher rates of transposition when expressed in mammalian in vitro and in vivo systems compared to the classical PBase at similar protein levels. Here we demonstrate that the usage of helper plasmids encoding the hyPBase irrespective of the codon-usage - also strikingly increases the rate of successful germline transformation in the Mediterranean fruit fly (Medfly) Ceratitis capitata, the red flour beetle Tribolium castaneum, and the vinegar fly Drosophila melanogaster. hyPBase-encoding helpers are therefore highly suitable for the generation of transgenic strains of diverse insect
\end{abstract}


orders. Depending on the species, we achieved up to 15-fold higher germline transformation rates compared to PBase and generated hard to obtain transgenic $T$. castaneum strains that express constructs affecting fitness and viability. Moreover, previously reported high sterility rates supposedly caused by hyPBase (iPB7), encoded by ${ }^{i} h y P B a s e$, could not be confirmed by our study. Therefore, we value hyPBase as an effective genetic engineering tool that we highly recommend for insect transgenesis.

- Keywords: Coleoptera; molecular entomology; Tephritid fruit flies; transgenics; transposon.

\section{Introduction}

Class II DNA transposases are enzymes that are utilized as genetic tools based on their ability to translocate DNA fragments by a "cut-and-paste-like" mechanism. The piggyBac transposase (PBase), isolated from a mutant Baculovirus strain in the cabbage looper moth Trichoplusia ni (Cary et al., 1989; Fraser et al., 1995; Handler and Harrell, 1999), is widely recognized for its broad range of targetable species and its ability to integrate large DNA cargo (Ding et al., 2005; Kahlig et al., 2010; Li et al., 2011). piggyBac-based elements can be excised without leaving a footprint, thus restoring the genomic locus to its pre-transposition state of the original TTAA target sequence (Elick et al., 1996). These features opened new possibilities for successful manipulation of various mammalian somatic and stem cell lines (Saridey et al., 2009; Wang et al., 2008; Yusa et al., 2009) and successful germline transformation in a vast variety of species including the mouse (Ding et al., 2005) as well as species of holometabolous and hemimetabolous insects (Berghammer et al., 1999; Handler et al., 1998; Nakamura et al., 2010; Tamura et al., 2000). This versatility led to its use in a wide range of scientific fields such as insect pest and disease vector control (Fu et al., 2010; Schetelig et al., 2009), gene or enhancer trap experiments (Bonin and Mann, 2004; Horn et al., 2003), the induction of pluripotent stem cells (Woltjen et al., 2009), or gene therapy (Wilson et al., 2007). Another distinguishing quality of PBase is the possibility to generate chimeric fusion constructs with e.g. DNA-binding domains to target specific genomic loci without hampering the transposition efficiency (Owens et al., 2012).

Despite the fact that there have been substantial variations in the reported relative transposition efficiencies and germline transformation rates of PBase throughout diverse target species and cell lines, direct comparisons of PBase with other transposases - including the hyperactive versions SB11 and SB12 of the previously widely used transposase Sleeping Beauty (SB) affirmed PBase to be the most effective (Wilson et al., 2007; Wu et al., 2006). Moreover, applications in mammalian systems highly benefited from the adjustment of the codon-usage from the original - by default insect codon-based - ${ }^{i}$ PBase to a mammalian codon-optimized ${ }^{m}$ PBase version of the piggyBac coding sequence (CDS). This elevated the transposition 
efficiency up to twenty times due to increased levels of PBase protein (Cadiñanos and Bradley, 2007). Furthermore, Yusa et al. (2011) generated a hyperactive version of PBase termed hyPBase, which carries seven amino acid substitutions that were implemented into the ${ }^{m} P B a s e$ CDS background ( $\left.{ }^{m} h y P B a s e\right)$. Expression of ${ }^{m} h y P B a s e$ in mouse embryonic stem cells demonstrated an additional tenfold increase in the transposition rate when compared to ${ }^{m}$ PBase (Yusa et al., 2011). Besides, examination of ${ }^{m}$ hyPBase, and the insect codon-based equivalent ihyPBase, confirmed the hyperactivity when compared to their wild-type counterparts for several human cell lines in vitro and mouse liver cells in vivo (Burnight et al., 2012; Doherty et al., 2012).

First results in our laboratory using ${ }^{m} h y P B a s e$ driven by the Drosophila melanogaster (Dm) heat-shock protein 70 regulatory region (Dm-hsp7g) (Lis et al., 1983) for germline transformation in the agricultural pest Ceratitis capitata $(C \mathcal{C})$ and the storage pest and emerging coleopteran model organism Tribolium castaneum $(T c)$, supported the literature and gave rise to approximately fourfold elevated transformation rates. This effect was even more prominent when utilizing the $T$. castaneum endogenous heat-shock protein 68 upstream region ( $T c$ hsp68) (Schinko et al., 2012) for germline transformation in T. castaneum (Dippel, 2016). Consequently, hyPBase has been established in our laboratory as a standard tool. To our surprise, the publication by Wright et al. (2013) reported low transformation efficiency and high sterility rates when co-injecting the ${ }^{i} h y P B a s e$ helper for germline transformation in Drosophila melanogaster and Aedes aegypti.

Because of these disparate experiences to deploy hyPBase for transgenesis in insects, we decided to conduct a systematic comparison and analysis of the performance of expressed ${ }^{i} P B a s e,{ }^{i} h y P B a s e$, and ${ }^{m} h y P B a s e$ for germline transformation in C. capitata, T. castaneum, and D. melanogaster. Our data from seven large-scale injection-sets with various donor plasmids confirmed our initial observations of substantially increased transformation efficiencies and could not detect any correlations between the use of hyPBase and elevated sterility rates.

We decided to use the indices ${ }^{\mathbf{i}}$ or ${ }^{\mathbf{m}}$, respectively, to ease the discrimination of mammalian and insect codon-optimized coding sequences of the wild-type (PBase) or hyperactive (hyPBase) transposase protein. Various nomenclatures and abbreviations have been used in the published piggyBac research, which are summarized in the materials and methods

\section{Materials and methods}

\section{Nomenclature of wild-type and hyperactive piggyBac transposases and respective coding sequences}

The wild-type ${ }^{i} P B a s e$ CDS was isolated from the cabbage looper moth and is therefore by default insect codon-based and was previously also abbreviated as $p B a c$ (Handler and Harrell, 1999), PB, PBase (Ding et al., 2005), iPB (Cadiñanos and Bradley, 2007), iPBase (Yusa et al., 2011), $p B$ and $i p B$ (Doherty et al., 2012). 
${ }^{m} P B a s e$ is the mammalian codon-optimized version of ${ }^{i} P B a s e$ and both encode the same wildtype PBase protein. ${ }^{m}$ PBase was previously also abbreviated as $m P B$ (Cadiñanos and Bradley, 2007) $m p B$ (Doherty et al., 2012) and mPBase (Yusa et al., 2009, 2011).

${ }^{i} h y P B a s e$ stands for the insect codon-based CDS of the hyperactive transposase hyPBase and was previously also abbreviated as $i 7 p B$, i7piggyBac(Doherty et al., 2012) and iPB7(Burnight et al., 2012).

${ }^{m}$ hyPBase stands for the mammalian codon-optimized CDS of the hyperactive transposase hyPBase and was previously also abbreviated as hyPBase (Yusa et al., 2011), $m 7 p B$, m7piggyBac (Doherty et al., 2012) and hypPB (Burnight et al., 2012). Again, both ${ }^{i} h y P B a s e$ and ${ }^{m} h y P B a s e$ encode the same hyPBase transposase.

\section{Plasmid construction}

\section{Helper plasmids}

The NC-iPB7 plasmid containing the ${ }^{i} h y P B a s e$ CDS (Doherty et al., 2012; Burnight et al., 2012) was purchased from Transposagen Biopharmaceuticals, Inc. (Lexington, U.S.A.). Sequencing of the received plasmid revealed an undesired "eighth" mutation (V336A) that we re-mutated by site directed mutagenesis of whole plasmids as previously described (Laible and Boonrod, 2009), using the primers $i P B-7 \_C t o T_{-} F$ and $i P B-7 \_C t o T \_R$, prior to further usage of the CDS for subsequent cloning steps. Primer sequences are listed in Supplementary Table 1.

The helper plasmid $T c-{ }^{m} h y P B a s e\left(p S L-f a \_T c-h s p \_5{ }^{\prime} U T R \_{ }^{m} h y P B a s e \_3{ }^{\prime} U T R \_f a\right)$ was generated by cloning the ${ }^{\mathrm{m}} h y P B a s e$ CDS from $p C M V$-hyPBase (Yusa et al., 2011) with Kpnl/Xhol into pSL-fa_Tc-hsp68_5'3'UTR_fa (Schinko et al., 2012) between the T. castaneum heat-shock protein 68 upstream region including the 5' UTR and the 3'UTR. Furthermore, an undesired $\mathrm{Ncol}-$ site in the backbone of $T_{c-}{ }^{m} h y P B a s e$ was removed by $B s t \mathrm{BI} /$ SmaI digest, blunting with T4 DNA polymerase, and re-ligation with T4 DNA ligase. To create $T c-{ }^{i} h y P B a s e$ and $T c-{ }^{i} P B a s e$ corresponding transposase CDS was amplified with primers piggyBac-NcoiForł piggyBac-NotIrev from templates remutated_NC-iPB7 and phsp-pBac (Handler and Harrell, 1999), respectively, and was subsequently cloned with Ncol/Not into $T \mathrm{C}^{-m}{ }^{m} h y P B a s e$, replacing the ${ }^{m} h y P B a s e$ CDS.

The helper plasmid Dm- ${ }^{m} h y P B a s e$ was created by cloning the ${ }^{m} h y P B a s e$ CDS with EcoRI/Not into the plasmid pSLfaHSfa (Ramos et al., 2006) between the upstream region plus 5' and 3' UTR of the D. melanogaster heat-shock protein 70. Helper plasmids Dm${ }^{i} h y P B a s e$ and $D m-{ }^{i} P B a s e$ were cloned analogously to $T c-{ }^{i} h y P B a s e$ and $T c-{ }^{i} P B a s e$. 


\section{Donor plasmids}

The donor plasmid PKO1 ( $\left.p B a c\left\{3 x P 3-g T c v ; T c^{\prime} a T u b 1 P-T c^{\prime} H 2 A v-E G F P\right\} a f\right)$ was used without further modifications (Kitzmann, 2016). To generate the donor plasmid PK13 (pBac\{3xP3-gTcv;alphaTubP-GAP43-mcherry\}af), the chimeric CDS of the growth associated protein-43 fused to $m$ Cherrywas amplified via PCR from plasmid $p C S 2+\{G A P 43-$ $m$ Cherry (a kind gift from Jubin Kashef, University Medical Center of Goettingen), using the primers PK124_FseIGAP43FW and PK122_mCherryAscIRV. The PCR product was subsequently cloned with $A s c \mathrm{I} / F s$ I into $P K 01$, between the $T c-\alpha-t u b u l i n 1$ promoter (5') and the SV4OPolyA site (3').

Plasmids pMK007 and KNE006 were assembled with the In-Fusion $® H D$ Cloning System (Takara Bio Europe/ Clontech., Stain-Germain-en-Laye, France) according to the manufacturer's protocol. To create plasmid pMKO07 (pBac\{3xP3-DsRed_5xQUAS$t G F P\}$ ), the $Q U A S$ and $t G F P C D S$ were amplified from $p Q U A S T$ (Potter et al., 2010) and $p S L-f a \_U A S \_T c-b h s p \_t G F P$ (Schinko et al., 2010) with primers QUAS_Fl QUAS_R and $H s p 68 \_F / t G F P \_R$, respectively. The two fragments were then cloned simultaneously into the AvrI-linearized plasmid \#707 pBac $\{3 x P 3-D s R e d>a f>\}$ (Horn et al., 2003) via the In-

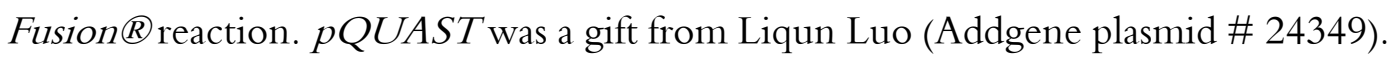

To generate plasmid KNE006 (pBac\{attP_TREhs43-mCherry_PUbEGFP\}), the TREhs43 and mCherry_SV4O fragments were amplified from KNEOOS pJFRC_20xUAS_Actin5c_mCherry_SV40 and \#1402 pBac\{fa_attP_TREhs43ATGCctra-hidAla5_PUb-nls-EGFP) (Ogaugwu et al., 2013) with primers IF_TRE_hs43_Fl IF_TRE_hs43_KS_R and IF_Cherry_SV4O_F/ IF_Cherry_SV4O_R, respectively. The two fragments were then cloned simultaneously into the $A s c$-linearized

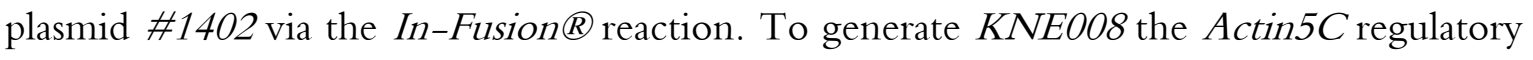
region and $m$ Cherry fragment were amplified from $p A C-G A L 4$ (Potter et al., 2010) and pcDNA3.1/hChR2(H134R)-mCherry (Zhang et al., 2007) with primers IC102/IC83 and IC86/IC91, respectively. The two fragments were then cloned simultaneously into the AatII/ BamHI linearized plasmid pJFR C7-20XUAS-IVS-mCD8::GFP(Pfeiffer et al., 2010) via the

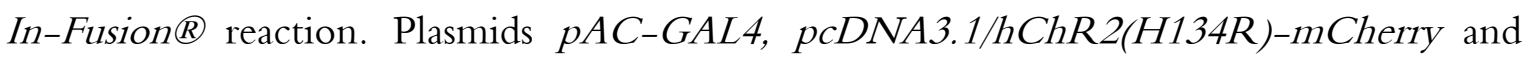
pJFRC7-20XUAS-IVS-mCD8::GFP were gifts from Liqun Luo (Addgene plasmid \# 24344), Karl Deisseroth (Addgene plasmid \# 20938), and Gerald Rubin (Addgene plasmid \# 26220), repectively.

To create plasmid KNEOOT (pXLII\{Dmß2tubulin-tTA_PUb-DsRed_attP\}), the $D$. melanogaster B2-tubulin upstream region plus 5'UTR was PCR amplified from $p C R I I[B 2-$ tubulinP] (Michiels et al., 1989) with primers B2Tub-F/ B2Tub-R, which added an AvrI and $X b a \mathrm{I}$ cut site to the 5' and 3' of the amplicon, respectively. The AvrII_Dmß2tubulin_XbaI fragment and \#437 pXLII_attP_PUb-DsRedT3_Ccvas-tTA (Schetelig and Handler, 2013) plasmid were restriction digested with $A v r \mathrm{I} / X b a \mathrm{I}$ and 
subsequently ligated. \#437 was a gift from Marc Schetelig (Justus-Liebig-University Giessen, Germany).

For $K N E 017$ ( $p X L I I\{A C P 70 A-t T A$; PUb-DsRed_attP $\}$ ), the $A C P 70 A$ upstream region plus 5'UTR was amplified from genomic DNA with primers AP70AgeIF/ AP70NheIIR which added an AgeI and NheI cut-site to the 5' and 3' of the amplicon, respectively. The AgeI_Dm-ACP7OA_NheI fragment and KNE007 plasmid were restriction digested with AgeI and Nhel and subsequently ligated.

To generate \#1413 (pBac\{Ccß2tubulin-tTA_PUb-DsRed\}), the Ccß2tubulin-tTA fragment was excised with $B g \Lambda \mathrm{I} / A s c$ from \#1412 $p S L \_C c \beta 2 t u b u l i n-t T A$ and subsequently ligated into $B g \Pi \mathrm{I} / A s c \mathrm{I}$ cut \#1200 pBac\{fa_PUb-DsRed\} (Scolari et al., 2008). To create \#1412, the Ccß2tubulin upstream region plus 5' UTR was PCR amplified from \#1228 pSLaf_CcB2t-tGFP-SV40_af(Scolari et al., 2008) with primers co109/ co110, which added an $\mathrm{NcO}$ and $\mathrm{XbaI}$ cut-site to the 5' and 3 ' of the amplicon, respectively. The NCOI_CC-B2tubulin_XbaI fragment was then cloned with $N c o I / X b a I$ into the plasmid \#1225 pSLaf_srya2-tTA-SV4O_af(Schetelig, 2008).

\section{DNA preparation}

Helper and donor plasmids were precipitated individually. To $90 \mu \mathrm{l}$ of an aqueous plasmid solution, containing $50 \mu \mathrm{g}$ DNA, $10 \mu \mathrm{l} 3 \mathrm{M} \mathrm{NaAc}$ and $900 \mu \mathrm{l}$ ice-cold EtOH were added and incubated over night at $-80^{\circ} \mathrm{C}$. Following centrifugation $\left(16000 \mathrm{rcf}, 4^{\circ} \mathrm{C}, 30 \mathrm{~min}\right)$ the DNA pellet was washed with 70\% ice-cold EtOH and again centrifuged (16000 rcf, $4^{\circ} \mathrm{C}, 15 \mathrm{~min}$ ), before being dried and redesolved in de-ionized $\mathrm{H}_{2} \mathrm{O}$. Helper and donor plasmids were mixed in a ratio of $300 \mathrm{ng} / \mu \mathrm{l}$ to $500 \mathrm{ng} / \mu \mathrm{l}$, respectively, in $1 \mathrm{x}$ injection buffer $(5 \mathrm{mM} \mathrm{KCl}, 0.1 \mathrm{mM}$ $\mathrm{NaH}_{2} \mathrm{PO}_{4}$, ph 6.8, 5\% phenol red) (Bachmann and Knust, 2008) and filtered using Millex®_ HV $0.45 \mu \mathrm{m}$ (Merck Millipore, Billerica, U.S.A.).

\section{Insect strains}

Experiments in C. capitata were conducted with the Egypt-II (EgII) wild-type strain which was obtained from the FAO/IAEA Agriculture and Biotechnology Laboratory (Entomology Unit, Seibersdorf, Austria). For experiments in $T$. castaneum, the white-eyed Tcvermillion ${ }^{\text {white }}\left(\mathrm{V}^{W}\right)$ strain was used (Lorenzen et al., 2002), whereas experiments regarding $D$. melanogaster were performed in the Oregon- $R$ wild-type strain. All strains of the different species were maintained under their respective standard relaxed artificial rearing conditions (Brown et al., 2009; Roberts, 1998; Saul, 1982).

\section{Germline transformation}

All experiments - injection, screening, and recording of the data - were performed doubleblind regarding which helper plasmid was used in the injection subsets. To reduce the impact of technical errors, which could induce additional lethality and sterility, we decided to inject only moderate volumes into the embryos. This is in contrast to experiments that do not aim 
for a systematic comparison but only seek for the highest possible transformation rate with the minimum effort for subsequent crossing and screening.

\section{Germline transformation in Ceratitis capitata and Drosophila melanogaster}

Germline transformation in C. capitata and D. melanogaster was carried out based on the principles of the previously described procedures (Bachmann and Knust, 2008; Handler et al., 1998; Spradling and Rubin, 1982) unless stated otherwise. Embryos were collected in a time interval of 30 minutes and were subsequently de-chorionated for 3 minutes in a $50 \%$ Klorix solution containing 2.5\% sodium hypochlorite (DanKlorix, CP GABA GmbH, Hamburg, Germany), briefly washed in washing buffer (100 mM NaCl, 0.02\% Triton X-100), washed thoroughly with double-deionized $\mathrm{H}_{2} \mathrm{O}$ and left for 5-10 min in double-deionized $\mathrm{H}_{2} \mathrm{O}$ to increase the internal pressure, before being fixed on double sided tape (Scotch ${ }^{\mathrm{TM}} \mathrm{Brand} / 3 \mathrm{M}$, St. Paul, USA) and covered with a thin layer of Voltalef 10 S oil (Lehmann \& Voss \& Co., Hamburg, Germany). Microinjections were performed using a FemtoJet ${ }^{\circledR}$ Microinjector (Eppendorf, Hamburg, Germany) and needles made from $10 \mathrm{~mm}$ x $1 \mathrm{~mm}$ quartz capillaries (Sutter Instrument, Novato, U.S.A.) using a P-2000 micropipette puller (Sutter Instrument, Novato, U.S.A.) applying the following settings: Heat $=750$, Fil $=4$, Vel $=50$, Del $=125$, $\mathrm{PUL}=175$. Needles were opened and sharpened using a microelectrode beveler (Bachofer $\mathrm{GmbH}$, Reutlingen, Germany). All steps post embryo collection were done at $18^{\circ} \mathrm{C}$. After injection, the oil was partially drained and $D$. melanogaster embryos were kept until hatching at $25^{\circ} \mathrm{C}$ and C. capitata embryos at $18^{\circ} \mathrm{C}$. A subset of D. melanogaster embryos were heatshocked twenty hours after injection at $37^{\circ} \mathrm{C}$ for 1 hour. Hatched larvae were transferred into the respective larval diet at the respective temperature according to standard laboratory rearing conditions.

\section{Germline transformation in Tribolium castaneum}

Germline transformation in $T$. castaneum was carried out based on the principles of the previously described procedure (Berghammer et al., 1999). Embryos were collected in a time interval of 1 hour and kept for one more hour at RT. The up to two-hours-old embryos were washed twice for 30 seconds in a $1 \%$ Klorix solution (equivalent to $0.05 \%$ sodium hypochlorite) (DanKlorix, CP GABA GmbH, Hamburg, Germany), and let dry for 5 minutes after aligning them into a row. Microinjection was performed using a FemtoJet ${ }^{\circledR}$ Microinjector (Eppendorf, Hamburg, Germany) and needles made from $10 \mathrm{~mm}$ x $1 \mathrm{~mm}$ borosilicate capillaries (Hilgenberg GmbH, Malsfeld, Germany) using a P-2000 micropipette puller (Sutter Instrument, Novato, U.S.A.) applying the following settings: Heat $=350$, Fil= 4, $\mathrm{Vel}=50, \mathrm{Del}=225, \mathrm{PUL}=150$. Needles were opened and sharpened using a microelectrode beveler (Bachofer $\mathrm{GmbH}$, Reutlingen, Germany). All steps post embryo collection were done at RT. After injection, the embryos were placed onto an apple agar plate in a sealed box at $30^{\circ} \mathrm{C}$ for 72 hours. Hatched larvae were transferred into vials with whole-wheat flour at $30^{\circ} \mathrm{C}$ according to standard laboratory rearing conditions. 


\section{Crossings and screening}

Irrespective of the species, $\mathrm{G}_{0}$ animals were backcrossed individually to three virgin animals of the opposite sex. Crosses were monitored regularly to differentiate between sterile $\mathrm{G}_{0}$ animals and those that have died. $\mathrm{F}_{1}$ offspring were anesthetized with $\mathrm{CO}_{2}$ and screened under a Leica M205 FA fluorescent stereo microscope (Leica, Wetzlar, Germany).

\section{Inverse PCR and sequence analysis}

To determine the genomic location of the piggyBac insertions and investigate for possible multiple insertions, inverse PCR (iPCR) was in principle carried out as described (Horn et al., 2003; Huang et al., 2000). The following modifications were introduced: genomic DNA was purified from approx. 10 adult D. melanogaster flies of each tested line, using the NucleoSpin ${ }^{\circledR}$ DNA Insect Kit (Macherey-Nagel GmbH \& Co. KG, Düren, Germany); isolated genomic DNA was restriction digested with $M s p I$ or $M b o I$ for the 5' junction and Bst or HindII for the 3' junction, respectively (Supplementary Table 2). Resulting DNA fragments were self-ligated and circularized DNA was used as template for the first PCR with primers $i P C R \_5 p B a c \_F /$ iPCR_5pBac_R for the 5' junction and primers $i P C R \_3 p B a c \_F /$ $i P C R \_3 p B a c \_R$ for the 3 ' junction. An aliquot of this first PCR reaction served as direct template for subsequently conducted nested PCRs, carried out with primers iPCR_5pBac_F_nested/ iPCR_5pBac_R_nested and primers iPCR_3pBac_F_nested/ iPCR_3pBac_R_nested, respectively (Supplementary Table 1). Distinct DNA bands were obtained by electrophoresis and DNA was purified from cut gel slices using the NucleoSpin ${ }^{\circledR}$ Gel and PCR Clean-up Kit (Macherey-Nagel GmbH \& Co. KG, Düren, Germany). The isolated DNA was Sanger-sequenced with either primer 5'-PB-SEQ for the 5' junction or 3'-PB-SEQ for the 3' junction (Supplementary Table 1). Sequences were used for BLAST search against the D. melanogaster genome (genome version: dmel_r6.20_FB2018_01; http://flybase.org/blast/).

\section{Results}

For a systematic comparative analysis of expressed ${ }^{i} P B a s e,{ }^{i} h y P B a s e$ and ${ }^{m} h y P B a s e$ in germline transformation, we generated two independent sets of the three helper plasmids for coinjection along with piggyBac donor plasmids in either C. capitata and D. melanogaster or $T$. castaneum. To be able to offer an objective comparison, helper plasmids within one set are identical except for the respective transposase CDS. For embryonic injections in Ceratitis and Drosophila we used the same set of helper plasmids which all have the Dm-hsp70 upstream region driving the expression of the transposase, whereas the Tribolium helper possess the endogenous $T c-h s p 68$ upstream region. To enable reliable screening for transgenic $F_{1}$ offspring, all donor plasmids contained a fluorescent eye or body marker. 


\section{Performance of the hyperactive piggyBac transposase variants in $C$. capitata}

The analysis of the germline transformation experiments conducted with the three different helper plasmids $D m-{ }^{i} P B a s e, D m-{ }^{i} h y P B a s e$, and $D m-{ }^{m} h y P B a s e$ in combination with either of the different donor plasmids (\#1413 and KNE006) in C. capitata (Table 1) revealed a substantially increased transformation rate (Figure 1A) for the experiments with both helper plasmids encoding the hyPBase (12.5\% to $16 \%$ ) compared to the wild-type PBase (0\%). The codon usage of the hyperactive piggyBac helper plasmids had no significant influence on its performance. Furthermore, in all experiments about two-thirds (62.5\% to $72.4 \%$ ) of the flies reaching adulthood produced offspring, with no obvious differences between the different helper plasmids (Figure 1B, n=228), which indicates no influence of the hyperactive piggyBac on the fertility rate.

\section{The activity of the hyperactive piggyBac variants in $T$. castaneum}

The systematic analysis of the germline transformation experiments in $T$. castaneum Table 2), which were performed using the helpers $T c{ }^{i} P B a s e, T c-{ }^{i} h y P B a s e$, and $T c-{ }^{m} h y P B a s e$ in combination with either of the three different donor plasmids (PK13, PK01, and pMK007), showed a substantial and at least doubled increase in the transformation rate by the hyPBase helpers $(17.6 \%$ to $39.4 \%)$ compared to the wild-type PBase ( $0 \%$ to $15 \%)$, and therefore confirmed our preliminary observations as well as the results from C. capitata. Interestingly, we found that both hyperactive helpers remarkably improved the transformation rate of constructs PK13 and PKO1, which were previously difficult to transform in our lab. Also in our initial experiment, we failed to generate transgenic beetles using the wild-type helper ( $T C$ ${ }^{i}$ PBase). Only by repeating one injection set (Figure 2A, PK13, second column) in a much larger scale, we eventually achieved a transformation rate of $1.6 \%$. In comparison to the over $20 \%$ transformation rate obtained with both hyperactive helpers, this clearly indicates an enormous improvement and offers the possibility to circumvent problems with constructs that are difficult to transform.

In $T$. castaneum we observed in contrast to $C$. capitata a slightly better performance using the helper with the insect codon-based hyperactive transposase CDS ( $\left.T c-{ }^{i} h y P B a s e\right)$.

Besides the much better performance of the hyPBase, we could not observe a negative effect on the survival or fertility rate in comparison to the wild-type PBase of the injected beetles (Figure 2B).

\section{The performance of the hyperactive piggyBac variants in D. melanogaster}

The systematic analysis of the germline transformation experiments conducted with the three different helper plasmids Dm- ${ }^{i} P B a s e, D m-{ }^{i} h y P B a s e$, and $D m-{ }^{m} h y P B a s$ in combination with either of the two donor plasmids (KNE007 and KNE017) and with or without heat-shock in $D$. melanogaster (Table 3) confirmed our previous results from the germline transformation experiments in T. castaneum and C. capitata. Under all conditions the two hyperactive helper 
variants outperformed the wild-type helper (Figure 3A), with a 3 to 11 -fold increased transformation rate compared to the wild-type helper. The codon usage of the hyPBase vectors had no consistent influence on the transformation rate. Heat-shock treatment however, consistently led to better performance of all tested helpers. As in the other species, also in $D$. melanogaster the fertility rate was not affected by the type of helper plasmid used (Figure 3B).

\section{hyPBase does not cause an increased rate of multiple insertions}

During the regular Drosophila crossings to determine the chromosomal localization of the diverse piggyBacinsertions, we did not find any indication for multiple insertions on different chromosomes for either of the three helper plasmids. To further investigate whether the enhanced germline transformation rate of hyPBase might result in an increased number of multiple insertion events on the same chromosome, we performed iPCR on ten lines generated by using the three different helper plasmids. In all cases, the 5 ' insertion sequence matches the 3' sequence (Table 4), which argues for single insertions. Therefore, we have no implication of an increased rate of multiple insertions for hyPBase compared to PBase that already had been shown to generate rare multiple insertions (Handler and Harrell, 1999).

\section{Discussion}

Our results clearly show that the hyperactive piggyBactransposase hyPBase, regardless of the codon-usage, decidedly increases the rate of successful germline transformation compared to the wild-type piggyBactransposase PBase in all three tested insect species. In C. capitata, we were not able to produce any transgenic offspring using $D m{ }^{i} P B a s e$ at the scale of our experimental setup but reached transformation rates of up to $14.3 \%$ or $16.0 \%$ deploying $D m$ ${ }^{m}$ hyPBase or Dm- ${ }^{i} h y P B a s e$, respectively. In D. melanogaster, we achieved a 3 - to 11 -fold increase in germline transformation when using $D m-{ }^{i} h y P B a s e$ and 5- to 8-times higher transformation efficiencies when co-injecting the $D m_{-}{ }^{m} h y P B a s e$ helper plasmid than with Dm-iPBase. However, in C. capitata and D. melanogaster we could not observe a constant trend towards a better performance for either of the codon-usages (Figure 1 and 3), indicating similarly effective translation of both codon-variants. Only in T. castaneum, the ${ }^{i} h y$ PBase helper showed consistently a slightly higher transformation efficiency than ${ }^{m} h y P B a s e$, where with 39.4\% ( $\left.T c-^{i} h y P B a s e\right)$ and $36.4 \%$ ( $\left.T c^{-}{ }^{m} h y P B a s e\right)$ also the highest transformation rates were obtained in our study (Figure 2). Actually, our results - especially the higher transformation rates of heat-shocked compared to not heat-shocked $D$. melanogaster embryos (Figure 3) - are consistent with the current opinion that piggyBac transposases lack the phenomenon of overproduction inhibition (Burnight et al., 2012; Cadiñanos and Bradley, 2007; Wilson et al., 2007).

Considering that $C$. capitata, D. melanogasterand T. castaneumbelong to two different insect orders and three different families, it is reasonable to speculate that similar improvements for 
germline transformation could also be achieved applying hyPBase to a variety of other insect species. This might be of particular interest when targeting non-model organisms, since their more elaborated handling during and after the injection procedure is often very labor intensive due to the species-specific biology, sensitivity to micro-manipulation and laborious artificial rearing conditions. In $C$. capitata for instance, successful individual backcrossing is one of the most restrictive steps since their polyandrous and complex mating behavior favors mating in larger populations (Bertin et al., 2010; Bonizzoni et al., 2006), which is directly represented in the lower average fertility rate in C. capitata of $67.4 \%$ compared to $96 \%$ in $T$. castaneum and $88 \%$ in $D$. melanogaster.

Furthermore, cytoplasmic localization of morphogenetic determinants at the posterior pole of the developing embryo are essential for germline formation and consequently for the fertility of the adult insect. Injuries at the posterior pole can result in the loss of germ cell development, followed by a decreased percentage of fertile survivors (Swanson and Poodry, 1980). In contrast to the injection directly into the tip of posterior pole in embryos of C. capitata and $D$. melanogaster, the injection from the lateral site at the posterior end in $T$. castaneum embryos seems less invasive and thus poses an additional factor for the observed high average fertility rates of the adult beetles. Therefore, we assume that sterility is mainly a consequence of the injection procedure itself, the injected volume, the degree of experience of the injecting person to prevent leakage, the mating behavior of the organism and a species-specific natural variation in fertility, rather than being a direct effect of the transposase as it has been previously hypothesized for the ${ }^{i} h y P B a s e$ helper (Wright et al., 2013).

In ordinary germline transformation experiments in our laboratory, we would usually inject higher volumes at the expense of lower survival rates and higher sterility rates. This can be convenient as it simultaneously reduces the workload on backcrossing and screening and increases the chance that survivors of this procedure will give rise to transgenic offspring. However, to be able to accurately evaluate the possible effects of the different transposases on survival and fertility rates in this systematic comparison, we decided to not exhaust the maximally injectable volume, allowing the speculation that even higher transformation rates could be achieved.

In conclusion, the main aim of our study was to systematically test the performance of the hyperactive piggyBac transposase compared to its wild-type version and further elucidate whether the codon usage could make a difference for germline transformation of insects. We found that the hyperactive variant indeed functions hyperactively in all respects but could not see a general strong favor in insects for either of the insect-based or mammalian codonoptimized version. Therefore, based on our findings, we encourage molecular entomologists to consider hyPBase for future germline transformation experiments. 


\section{Author Contributions}

E.A.W., K.N.E., and S.D. conceived and designed the research; S.D., K.N.E. and M.K. designed the helper plasmids; K.N.E., M.K., P.K. and C.E.O. constructed the plasmids; K.N.E., M.K. and H.M.M.A. designed the experiments; K.N.E, M.K., and H.M.M.A. performed the injections; K.N.E, M.K., H.M.M.A. and M.D.I performed the crossings and screenings; K.N.E, S.D. and E.A.W., wrote the manuscript; K.N.E prepared the figures and tables; All authors edited and approved the final version of the manuscript.

\section{Acknowledgments}

We would like to express our gratitude to Johannes B. Schinko (University of Goettingen), Marc Schetelig (Justus-Liebig-University Giessen), Jubin Kashef (University Medical Center Goettingen) and Renate Renkawitz-Pohl (Philipps-University Marburg) for providing plasmids. We thank Nancy L. Craig and the Welcome Trust Sanger Institute for making the $p C M V$-hypBase plasmid available and Ingrid M. Curril (University of Goettingen) for technical assistance.

Personal funding: H.M.M.A. and M.D.I. are supported by the German Academic Exchange Service (DAAD) and the Evangelisches Studienwerk Villigst, respectively. M.K. received a Stipend of the Excellence Foundation for the Promotion of the Max Planck Society. In addition, we would like to thank the department of Molecular Developmental Biology (Herbert Jäckle) at the MPI for Biophysical Chemistry for support.

Project funding. This research did not receive any specific grant from funding agencies in the public, commercial, or not-for-profit sectors. 


\section{References}

Bachmann, A., Knust, E., 2008. The Use of P-Element Transposons to Generate Transgenic Flies, in: Dahmann, C. (Ed.), Drosophila: Methods and Protocols, Methods in Molecular Biology. Humana Press, Totowa, N.J, pp. 61-77.

Berghammer, A.J., Klingler, M., A. Wimmer, E., 1999. Genetic techniques: A universal marker for transgenic insects. Nature 402, 370-371. doi:10.1038/46463

Bertin, S., Scolari, F., Guglielmino, C.R., Bonizzoni, M., Bonomi, A., Marchini, D., Gomulski, L.M., Gasperi, G., Malacrida, A.R., Matessi, C., 2010. Sperm storage and use in polyandrous females of the globally invasive fruitfly, Ceratitis capitata. J. Insect Physiol. 56, 1542-1551. doi:10.1016/j.jinsphys.2010.05.006

Bonin, C.P., Mann, R.S., 2004. A piggyBac Transposon Gene Trap for the Analysis of Gene Expression and Function in Drosophila. Genetics 167, 1801-1811. doi:10.1534/genetics.104.027557

Bonizzoni, M., Gomulski, L.M., Mossinson, S., Guglielmino, C.R., Malacrida, A.R., Yuval, B., Gasperi, G., 2006. Is Polyandry a Common Event Among Wild Populations of the Pest Ceratitis capitata? J. Econ. Entomol. 99, 1420-1429. doi:10.1603/0022-0493-99.4.1420

Brown, S.J., Shippy, T.D., Miller, S., Bolognesi, R., Beeman, R.W., Lorenzen, M.D., Bucher, G., Wimmer, E.A., Klingler, M., 2009. The Red Flour Beetle, Tribolium castaneum (Coleoptera): A Model for Studies of Development and Pest Biology. Cold Spring Harb. Protoc. 2009, pdb.emo126-pdb.emo126. doi:10.1101/pdb.emo126

Burnight, E.R., Staber, J.M., Korsakov, P., Li, X., Brett, B.T., Scheetz, T.E., Craig, N.L., McCray, P.B., 2012. A Hyperactive Transposase Promotes Persistent Gene Transfer of a piggyBac DNA Transposon. Mol. Ther. Nucleic Acids 1, e50. doi:10.1038/mtna.2012.12

Cadiñanos, J., Bradley, A., 2007. Generation of an inducible and optimized piggyBac transposon system. Nucleic Acids Res. 35, e87. doi:10.1093/nar/gkm446

Cary, L.C., Goebel, M., Corsaro, B.G., Wang, H.-G., Rosen, E., Fraser, M.J., 1989. Transposon mutagenesis of baculoviruses: Analysis of Trichoplusia ni transposon IFP2 insertions within the FP-locus of nuclear polyhedrosis viruses. Virology 172, 156-169. doi:10.1016/00426822(89)90117-7

Ding, S., Wu, X., Li, G., Han, M., Zhuang, Y., Xu, T., 2005. Efficient Transposition of the piggyBac (PB) Transposon in Mammalian Cells and Mice. Cell 122, 473-483. doi:10.1016/j.cell.2005.07.013

Dippel, S., 2016. Comprehensive Morphological and Transcriptomic Analysis of the Chemosensory System in the Red Flour Beetle, Tribolium castaneum. 
Doherty, J.E., Huye, L.E., Yusa, K., Zhou, L., Craig, N.L., Wilson, M.H., 2012. Hyperactive piggyBac gene transfer in human cells and in vivo. Hum. Gene Ther. 23, 311-320. doi:10.1089/hum.2011.138

Elick, T.A., Bauser, C.A., Fraser, M.J., 1996. Excision of the piggyBac transposable element in vitro is a precise event that is enhanced by the expression of its encoded transposase. Genetica 98, 3341. doi:10.1007/BF00120216

Fraser, M.J., Cary, L., Boonvisudhi, K., Wang, H.-G.H., 1995. Assay for Movement of Lepidopteran Transposon IFP2 in Insect Cells Using a Baculovirus Genome as a Target DNA. Virology 211, 397-407. doi:10.1006/viro.1995.1422

Fu, G., Lees, R.S., Nimmo, D., Aw, D., Jin, L., Gray, P., Berendonk, T.U., White-Cooper, H., Scaife, S., Phuc, H.K., Marinotti, O., Jasinskiene, N., James, A.A., Alphey, L., 2010. Femalespecific flightless phenotype for mosquito control. Proc. Natl. Acad. Sci. 107, 4550-4554. doi:10.1073/pnas.1000251107

Handler, A.M., Harrell, R.A., 1999. Germline transformation of Drosophila melanogaster with the piggyBac transposon vector. Insect Mol. Biol. 8, 449-457. doi:10.1046/j.13652583.1999.00139.x

Handler, A.M., McCombs, S.D., Fraser, M.J., Saul, S.H., 1998. The lepidopteran transposon vector, piggyBac, mediates germ-line transformation in the Mediterranean fruit fly. Proc. Natl. Acad. Sci. 95, 7520-7525.

Horn, C., Offen, N., Nystedt, S., Häcker, U., Wimmer, E.A., 2003. piggyBac-based insertional mutagenesis and enhancer detection as a tool for functional insect genomics. Genetics 163, 647661.

Kahlig, K.M., Saridey, S.K., Kaja, A., Daniels, M.A., George, A.L., Wilson, M.H., 2010. Multiplexed transposon-mediated stable gene transfer in human cells. Proc. Natl. Acad. Sci. 107, 1343-1348. doi:10.1073/pnas.0910383107

Kitzmann, P., 2016. Morphogenesis and Genetic Regulation of the Insect Head. Dissertation, GeorgAugust-University Göttingen.

Laible, M., Boonrod, K., 2009. Homemade Site Directed Mutagenesis of Whole Plasmids. J. Vis. Exp. JoVE. doi:10.3791/1135

Li, M.A., Turner, D.J., Ning, Z., Yusa, K., Liang, Q., Eckert, S., Rad, L., Fitzgerald, T.W., Craig, N.L., Bradley, A., 2011. Mobilization of giant piggyBac transposons in the mouse genome. Nucleic Acids Res. 39, e148-e148. doi:10.1093/nar/gkr764 
Lis, J.T., Simon, J.A., Sutton, C.A., 1983. New heat shock puffs and $\beta=$ galactosidase activity resulting from transformation of Drosophila with an hsp70-lacZ hybrid gene. Cell 35, 403-410. doi:10.1016/0092-8674(83)90173-3

Lorenzen, M.D., Brown, S.J., Denell, R.E., Beeman, R.W., 2002. Cloning and Characterization of the Tribolium castaneum Eye-Color Genes Encoding Tryptophan Oxygenase and Kynurenine 3-Monooxygenase. Genetics 160, 225-234.

Michiels, F., Gasch, A., Kaltschmidt, B., Renkawitz-Pohl, R., 1989. A 14 bp promoter element directs the testis specificity of the Drosophila beta 2 tubulin gene. EMBO J. 8, 1559-1565.

Nakamura, T., Yoshizaki, M., Ogawa, S., Okamoto, H., Shinmyo, Y., Bando, T., Ohuchi, H., Noji, S., Mito, T., 2010. Imaging of Transgenic Cricket Embryos Reveals Cell Movements Consistent with a Syncytial Patterning Mechanism. Curr. Biol. 20, 1641-1647. doi:10.1016/j.cub.2010.07.044

Ogaugwu, C.E., Schetelig, M.F., Wimmer, E.A., 2013. Transgenic sexing system for Ceratitis capitata (Diptera: Tephritidae) based on female-specific embryonic lethality. Insect Biochem. Mol. Biol. 43, 1-8. doi:10.1016/j.ibmb.2012.10.010

Owens, J.B., Urschitz, J., Stoytchev, I., Dang, N.C., Stoytcheva, Z., Belcaid, M., Maragathavally, K.J., Coates, C.J., Segal, D.J., Moisyadi, S., 2012. Chimeric piggyBac transposases for genomic targeting in human cells. Nucleic Acids Res. 40, 6978. doi:10.1093/nar/gks309

Pfeiffer, B.D., Ngo, T.-T.B., Hibbard, K.L., Murphy, C., Jenett, A., Truman, J.W., Rubin, G.M., 2010. Refinement of tools for targeted gene expression in Drosophila. Genetics 186, 735-755. doi:10.1534/genetics.110.119917

Potter, C.J., Tasic, B., Russler, E.V., Liang, L., Luo, L., 2010. The Q System: A Repressible Binary System for Transgene Expression, Lineage Tracing and Mosaic Analysis. Cell 141, 536-548. doi:10.1016/j.cell.2010.02.025

Ramos, D.M., Kamal, F., Wimmer, E.A., Cartwright, A.N., Monteiro, A., 2006. Temporal and spatial control of transgene expression using laser induction of the hsp70promoter. BMC Dev. Biol. 6, 55. doi:10.1186/1471-213X-6-55

Roberts, D.B. (Ed.), 1998. Drosophila: a practical approach, 2nd ed, The practical approach series. Oxford [u.a.] : IRL Press, 1984-. IRL Press at Oxford Univ. Press, Oxford [u.a.].

Saridey, S.K., Liu, L., Doherty, J.E., Kaja, A., Galvan, D.L., Fletcher, B.S., Wilson, M.H., 2009. PiggyBac Transposon-based Inducible Gene Expression In Vivo After Somatic Cell Gene Transfer. Mol. Ther. 17, 2115. doi:10.1038/mt.2009.234 
Saul, S.H., 1982. Rosy-like Mutant of the Mediterranean Fruit Fly, Ceratitis capitata (Diptera: Tephritidae), and Its Potential for Use in a Genetic Sexing Program. Ann. Entomol. Soc. Am. 75, 480-483. doi:10.1093/aesa/75.4.480

Schetelig, M., Handler, A., 2013. Y-Linked Markers for Improved Population Control of the Tephritid Fruit Fly Pest, Anastrepha suspensa, in: Vilcinskas, A. (Ed.), Yellow Biotechnology II, Advances in Biochemical Engineering/Biotechnology. Springer Berlin Heidelberg, pp. 123133. doi:10.1007/10_2013_209

Schetelig, M.F., 2008. New molecular technologies to improve the Sterile Insect Technique for the Mediterranean fruitfly Ceratitis capitata (Diptera; Tephritidae).

Schetelig, M.F., Caceres, C., Zacharopoulou, A., Franz, G., Wimmer, E.A., 2009. Conditional embryonic lethality to improve the sterile insect technique in Ceratitis capitata(Diptera: Tephritidae). BMC Biol. 7, 4. doi:10.1186/1741-7007-7-4

Schinko, J.B., Hillebrand, K., Bucher, G., 2012. Heat shock-mediated misexpression of genes in the beetle Tribolium castaneum. Dev. Genes Evol. 222, 287-298. doi:10.1007/s00427-012-0412$\mathrm{x}$

Schinko, J.B., Weber, M., Viktorinova, I., Kiupakis, A., Averof, M., Klingler, M., Wimmer, E.A., Bucher, G., 2010. Functionality of the GAL4/UAS system in Tribolium requires the use of endogenous core promoters. BMC Dev. Biol. 10, 53. doi:10.1186/1471-213X-10-53

Scolari, F., Schetelig, M.F., Bertin, S., Malacrida, A.R., Gasperi, G., Wimmer, E.A., 2008. Fluorescent sperm marking to improve the fight against the pest insect Ceratitis capitata (Wiedemann; Diptera: Tephritidae). New Biotechnol. 25, 76-84. doi:10.1016/j.nbt.2008.02.001

Spradling, A.C., Rubin, G.M., 1982. Transposition of cloned P elements into Drosophila germ line chromosomes. Science 218, 341-347. doi:10.1126/science.6289435

Swanson, M.M., Poodry, C.A., 1980. Pole cell formation in Drosophila melanogaster. Dev. Biol. 75 , 419-430. doi:10.1016/0012-1606(80)90173-6

Tamura, T., Thibert, C., Royer, C., Kanda, T., Eappen, A., Kamba, M., Kômoto, N., Thomas, J.L., Mauchamp, B., Chavancy, G., Shirk, P., Fraser, M., Prudhomme, J.-C., Couble, P., 2000. Germline transformation of the silkworm Bombyx mori L. using a piggyBac transposon-derived vector. Nat. Biotechnol. 18, 81-84. doi:10.1038/71978

Wang, W., Lin, C., Lu, D., Ning, Z., Cox, T., Melvin, D., Wang, X., Bradley, A., Liu, P., 2008. Chromosomal transposition of PiggyBac in mouse embryonic stem cells. Proc. Natl. Acad. Sci. 105, 9290-9295. doi:10.1073/pnas.0801017105 
Wilson, M.H., Coates, C.J., George Jr, A.L., 2007. PiggyBac Transposon-mediated Gene Transfer in Human Cells. Mol. Ther. 15, 139-145. doi:10.1038/sj.mt.6300028

Woltjen, K., Michael, I.P., Mohseni, P., Desai, R., Mileikovsky, M., Hämäläinen, R., Cowling, R., Wang, W., Liu, P., Gertsenstein, M., Kaji, K., Sung, H.-K., Nagy, A., 2009. piggyBac transposition reprograms fibroblasts to induced pluripotent stem cells. Nature 458, 766-770. doi:10.1038/nature07863

Wright, J.A., Smith, R.C., Xie, K., Craig, N.L., Atkinson, P.W., 2013. IPB7 transposase behavior in Drosophila melanogaster and Aedes aegypti. Insect Biochem. Mol. Biol. 43, 899-906. doi:10.1016/j.ibmb.2013.06.009

Wu, S.C.-Y., Meir, Y.-J.J., Coates, C.J., Handler, A.M., Pelczar, P., Moisyadi, S., Kaminski, J.M., 2006. piggyBac is a flexible and highly active transposon as compared to Sleeping Beauty, Tol2, and Mos1 in mammalian cells. Proc. Natl. Acad. Sci. 103, 15008-15013. doi:10.1073/pnas.0606979103

Yusa, K., Rad, R., Takeda, J., Bradley, A., 2009. Generation of transgene-free induced pluripotent mouse stem cells by the piggyBac transposon. Nat. Methods 6, 363-369. doi: $10.1038 /$ nmeth.1323

Yusa, K., Zhou, L., Li, M.A., Bradley, A., Craig, N.L., 2011. A hyperactive piggyBac transposase for mammalian applications. Proc. Natl. Acad. Sci. U. S. A. 108, 1531-1536. doi:10.1073/pnas.1008322108

Zhang, F., Wang, L.-P., Brauner, M., Liewald, J.F., Kay, K., Watzke, N., Wood, P.G., Bamberg, E., Nagel, G., Gottschalk, A., Deisseroth, K., 2007. Multimodal fast optical interrogation of neural circuitry. Nature 446, 633-639. doi:10.1038/nature05744 


\section{Figures:}
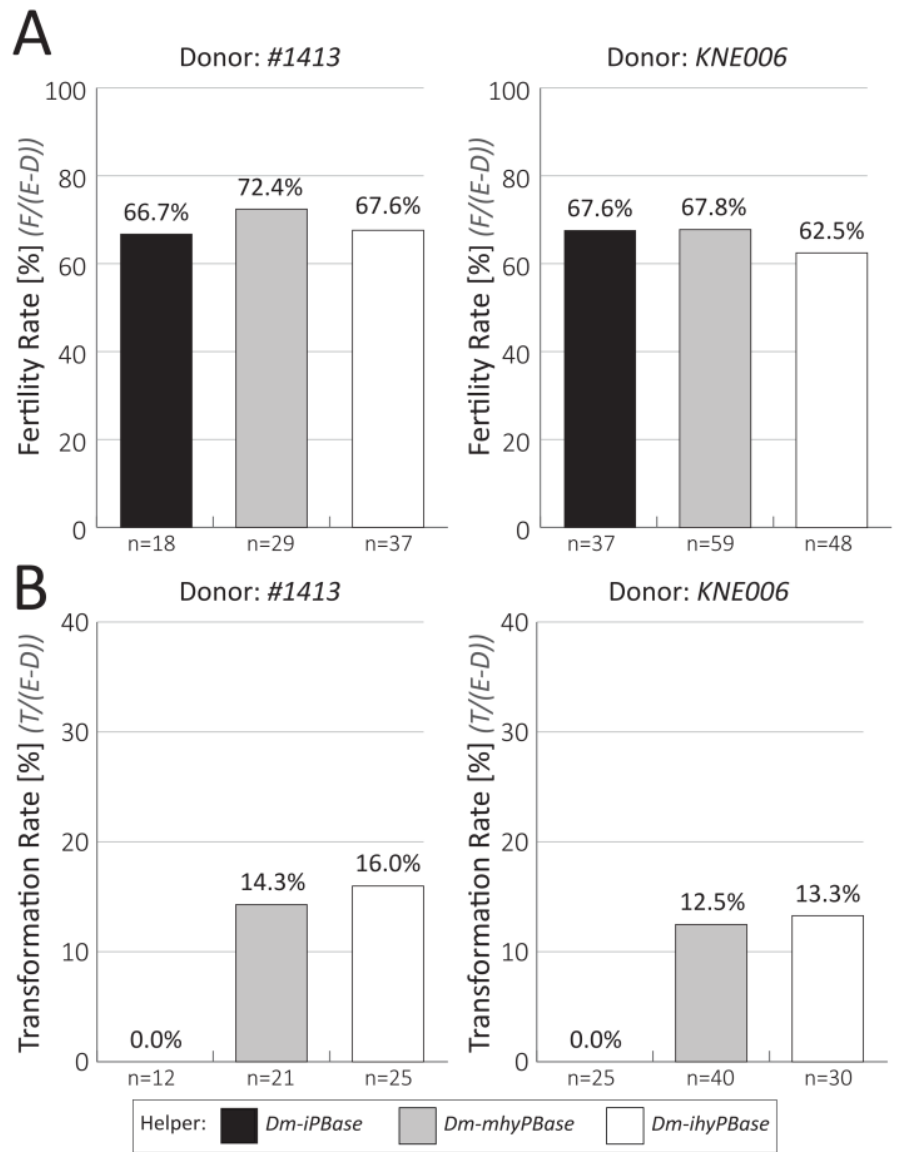

Figure 1 Transformation rate (A) and fertility rate (B) obtained from co-injection of three different helper plasmids (Dm- ${ }^{i} P B a s e$ (black), Dm- ${ }^{i} h y P B a s e$ (grey), and Dm- ${ }^{m} h y P B a s e$ (white)) in combination with two different donor plasmids (\#1413 and KNE006) in C. capitata. D, death; E, eclosed flies; F, fertile crosses; T, producing transgenic offspring). 

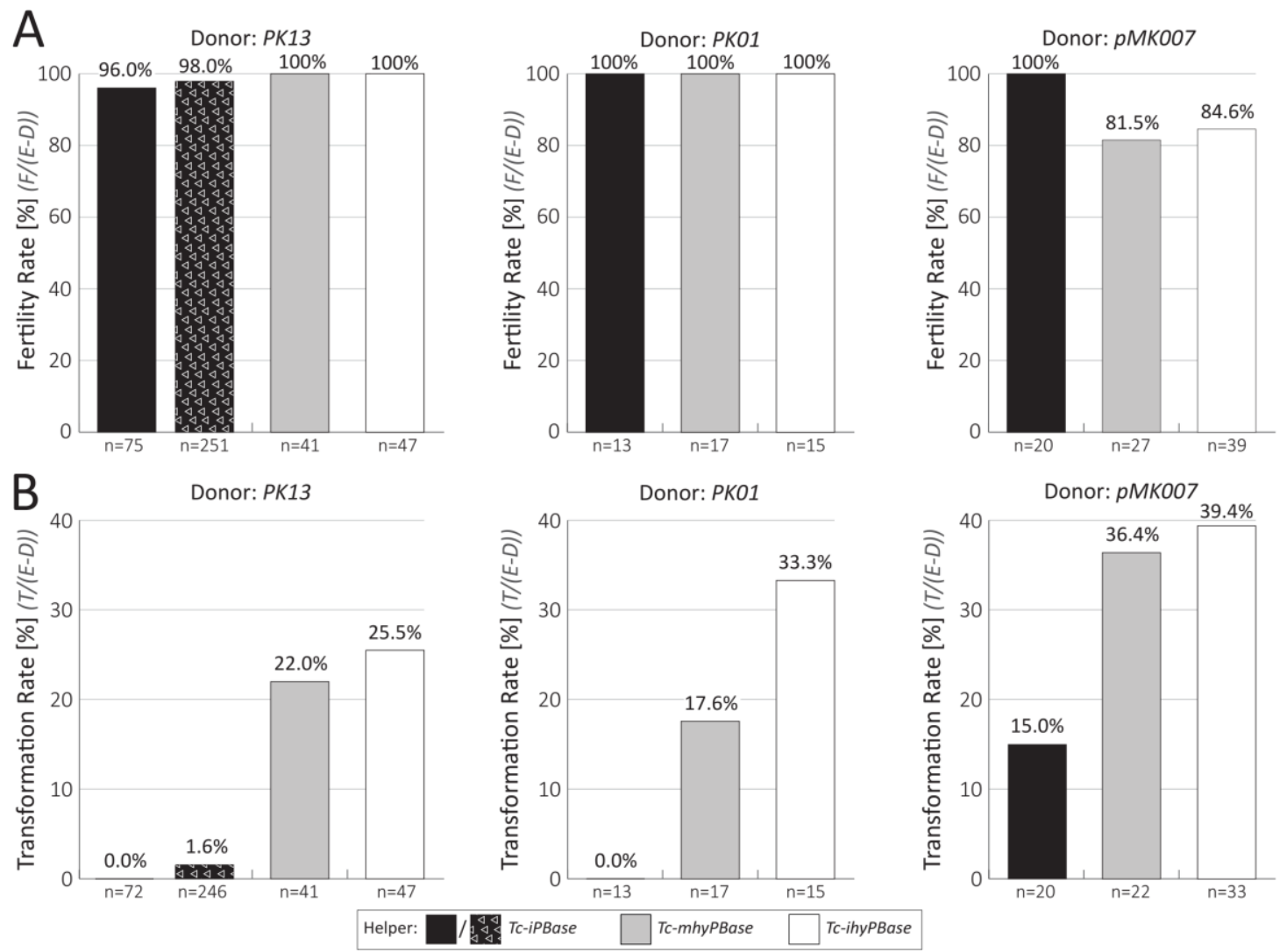

Figure 2 Transformation rate (A) and fertility rate (B) obtained from co-injection of three different helper

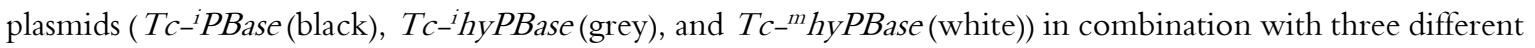
donor plasmids PK13, PK01, and pMKO07) in T.castaneum. D, death; E, eclosed beetles; F, fertile crosses; T, producing transgenic offspring). 
A

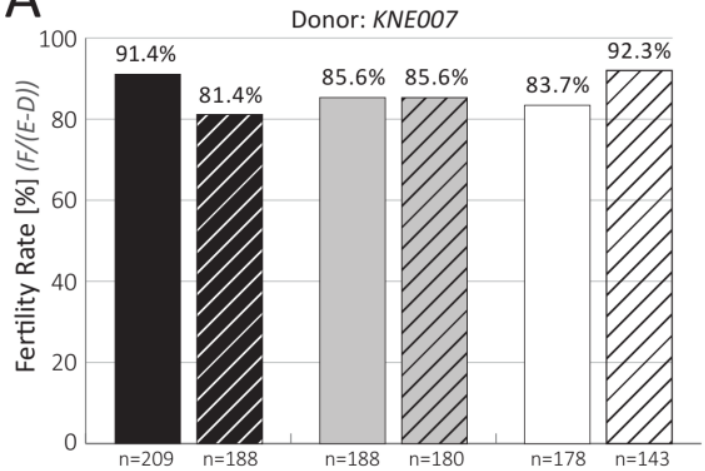

B

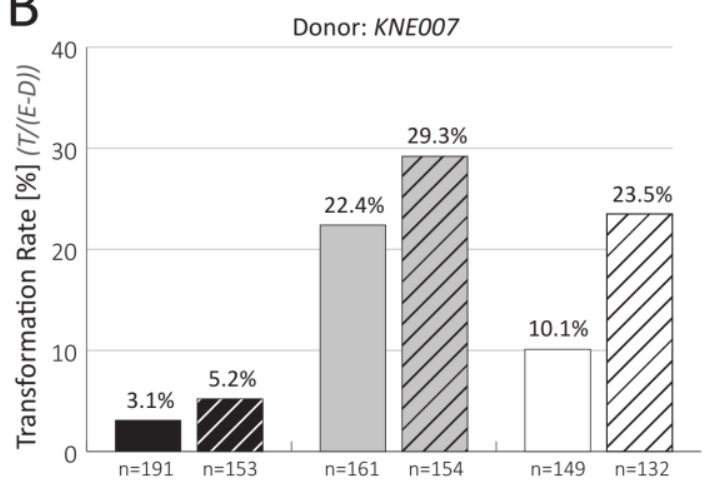

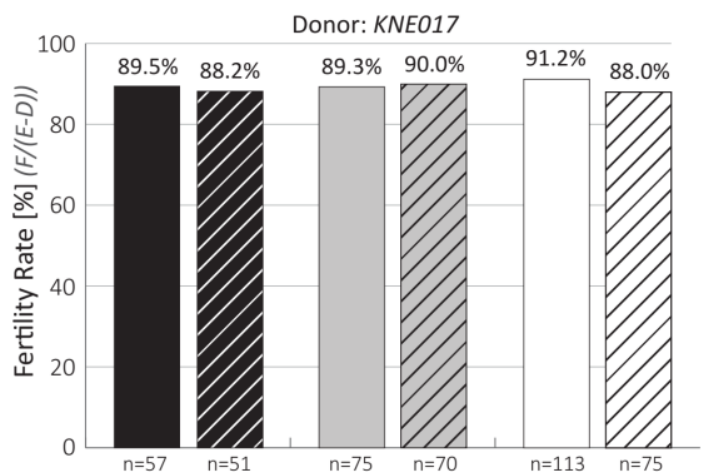

Donor: KNE017

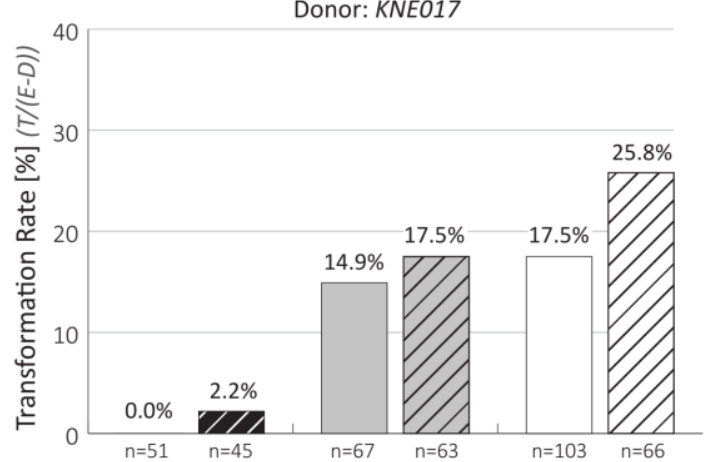

Figure 3 Transformation rate (A) and fertility rate (B) obtained from co-injection of three different helper plasmids (Dm- ${ }^{i}$ PBase (black), Dm- ${ }^{i} h y P B a s e$ (grey), and $D m-{ }^{m} h y P B a s e$ (white)) in combination with two different donor plasmids (KNEOO7 and KNE017) as well as without and with heat-shock (striped columns) in D. melanogaster. D, death; E, eclosed flies; F, fertile crosses; T, producing transgenic offspring). 
Table 1 Numbers of injected embryos, survivors and fertile crosses, and their respective rates, using the helpers $D m-$ ${ }^{i}$ PBase, Dm- ${ }^{i}$ hyPBase, and Dm- ${ }^{m}$ hyPBase in C. capitata.

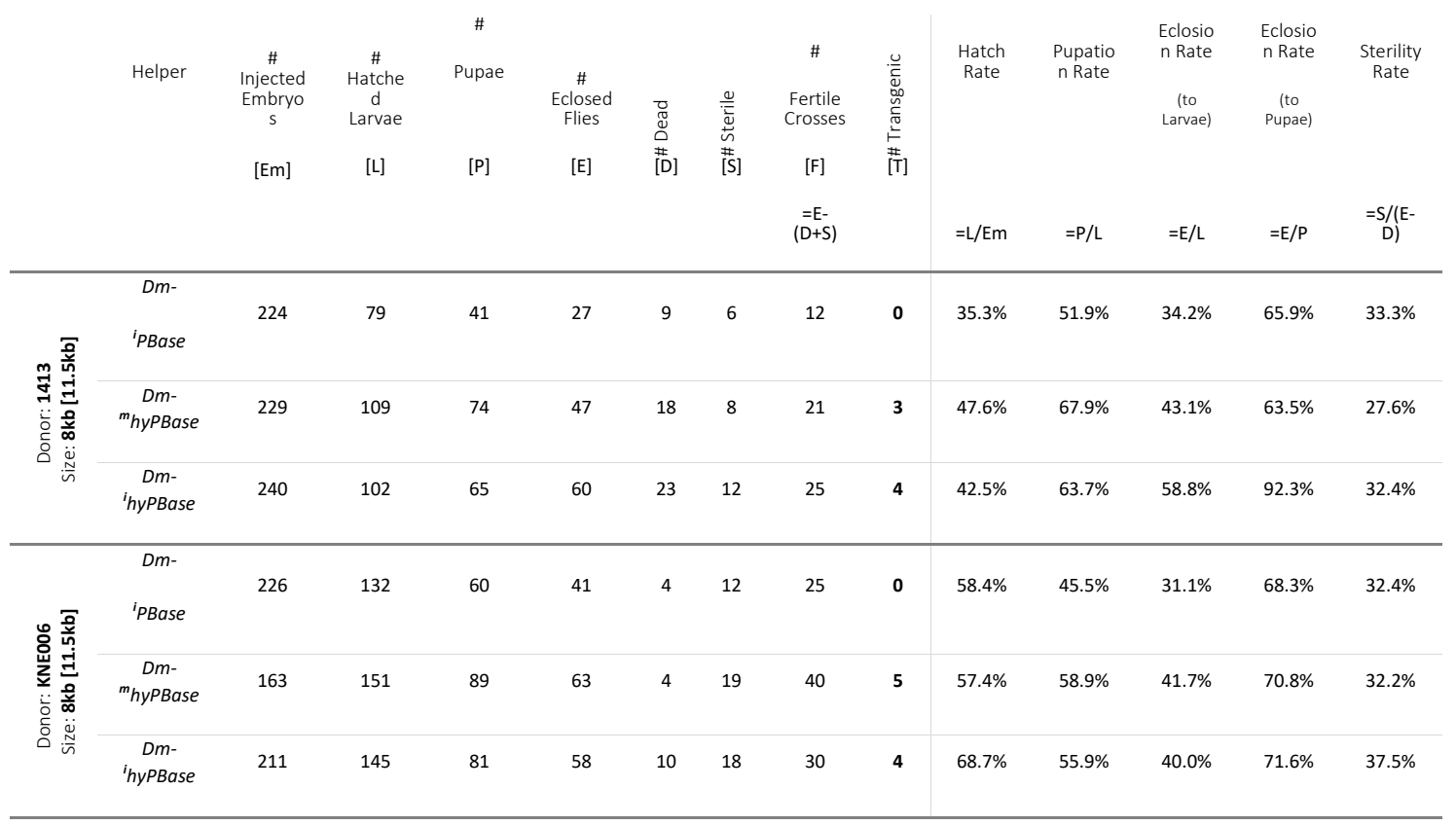


Table 2 Numbers of injected embryos, survivors, fertile crosses, and their respecitve rates, using the helpers $T c{ }^{i} P B a s e$, $\overline{T c-}{ }^{i} h y P B a s e$, and $T c_{-}{ }^{m} h y P B a s e$ in T. castaneum.

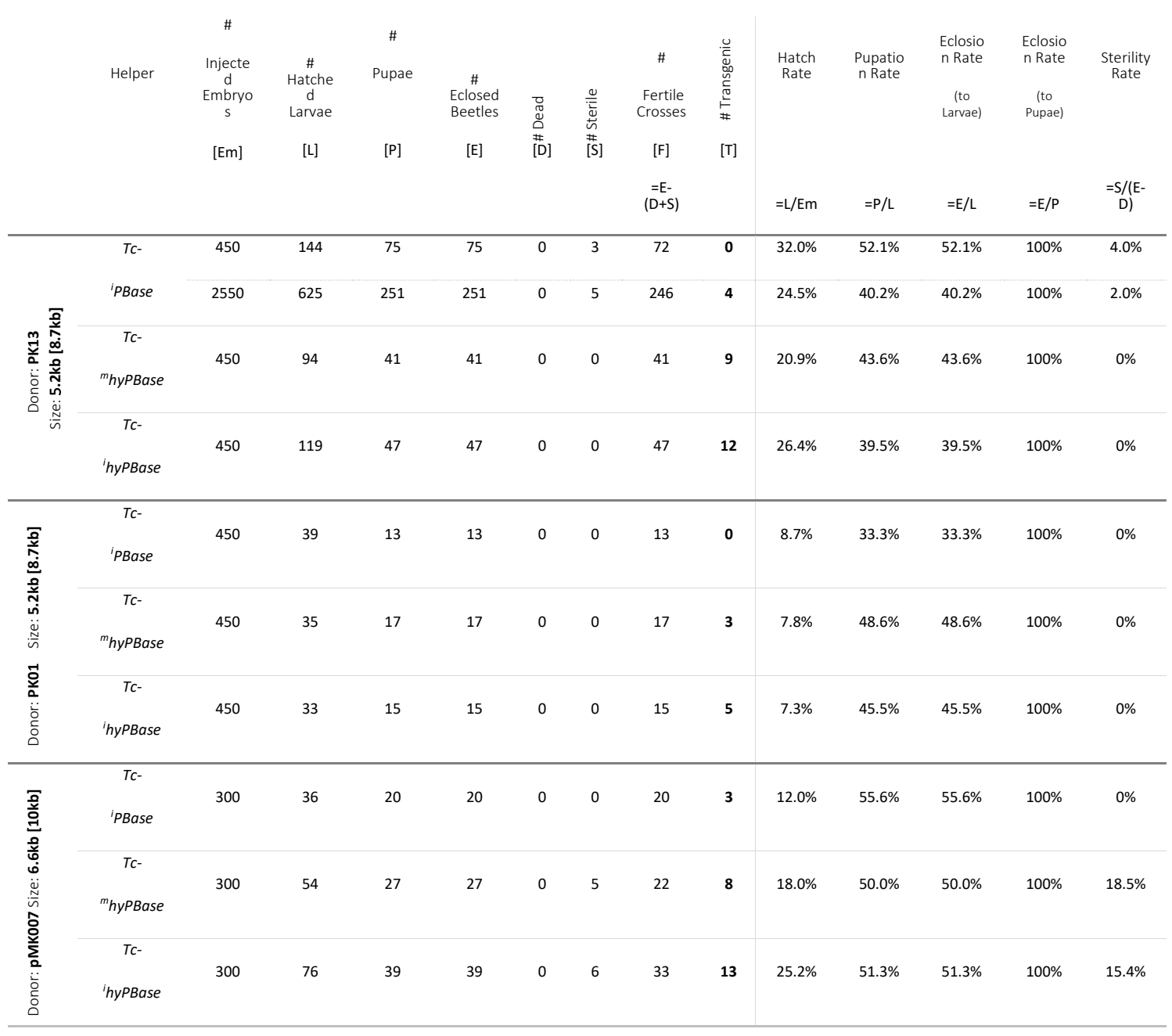


Table 3 Numbers of injected embryos, survivors, fertile crosses, and their respective rates, using the helpers $D m-{ }^{i} P B a s e$, $\overline{D m-}{ }^{i} h y P B a s e$, and $D m{ }^{m}{ }^{m} h y P B a s e$ in D. melanogaster (greyscale: heat-shock).

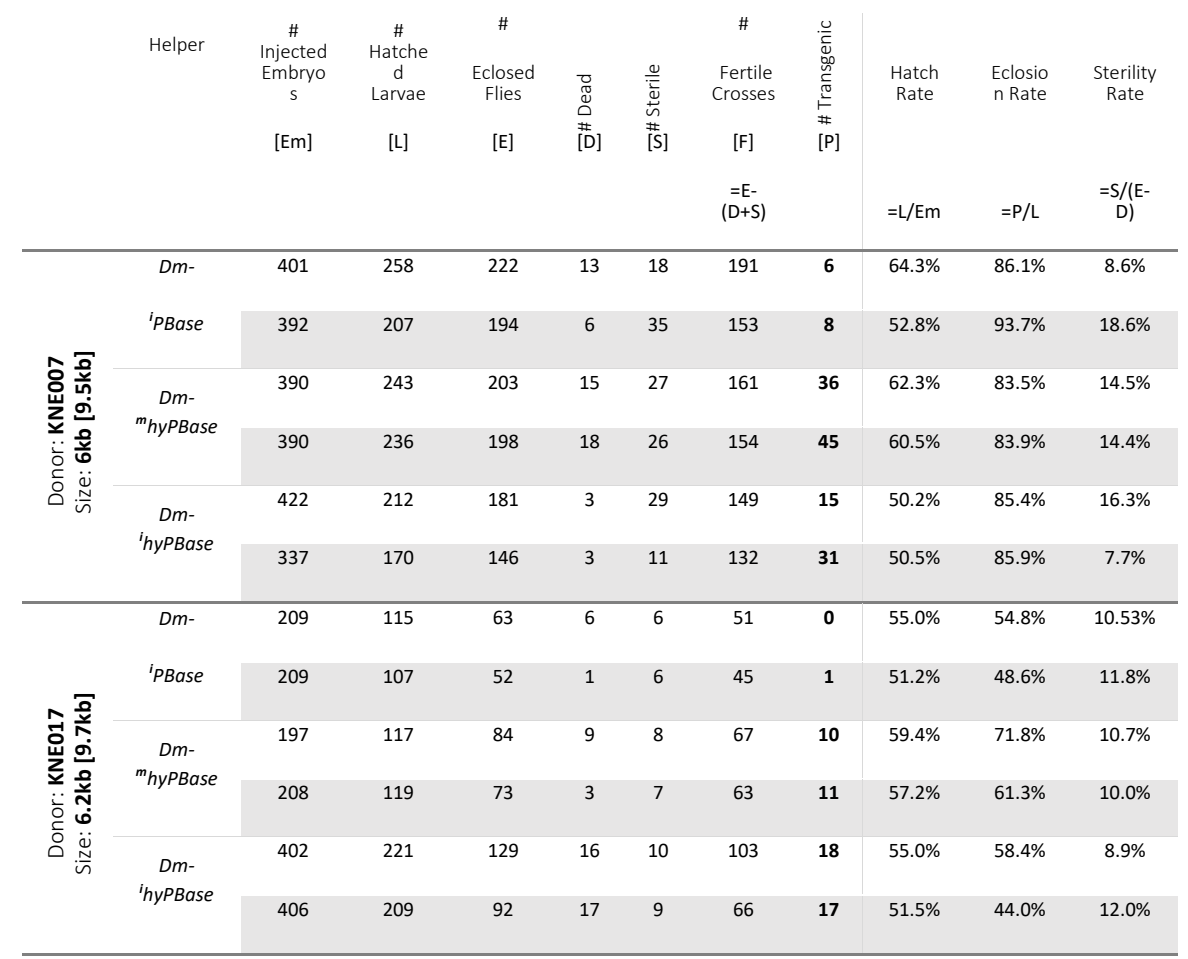


Table 4 Insertion-site sequences isolated by inverse PCR from sets of $D$. melanogaster lines which were generated by either of the three helper plasmids (Dm- ${ }^{i}$ PBase, Dm- ${ }^{i} h y P B a s e$ or $\left.D m-{ }^{m} h y P B a s e\right)$. In all ten examined lines only a single

\begin{tabular}{|c|c|c|c|c|}
\hline Donor & Helper & Line & Genomic 5' and 3' Sequence of piggyBac Insertion & $\begin{array}{l}\text { Genomic Locus } \\
\text { of Insertion }\end{array}$ \\
\hline \multirow{6}{*}{$\begin{array}{l}0 \\
\vdots \\
\dot{\omega} \\
z\end{array}$} & \multirow{3}{*}{$\begin{array}{c}D m- \\
{ }^{i} P B a s e\end{array}$} & 007.2 & 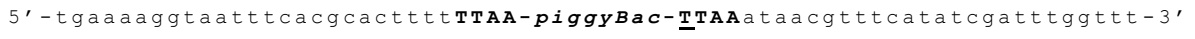 & $2 R: 11605849$ \\
\hline & & 007.4 & 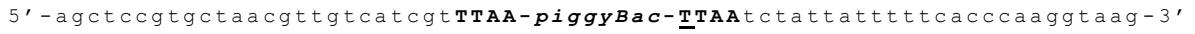 & $x: 10070165$ \\
\hline & & 007.7 & $5^{\prime}-$ tctttaagggtgagtgacttcattgTTAA-piggyBac- TTAAaggctttaccacatacactctgtga-3' & $3 \mathrm{~L}: 10473387$ \\
\hline & \multirow{3}{*}{$\begin{array}{c}\text { Dm- } \\
{ }^{m} \text { hyPBase }\end{array}$} & 007.3 & $5^{\prime}$-ttccagcatgctcacataattaactTTAA-piggyBac- TTAAataaaacagttgtaactatatat-3' & $2 \mathrm{R}: 17563360$ \\
\hline & & 007.6 & 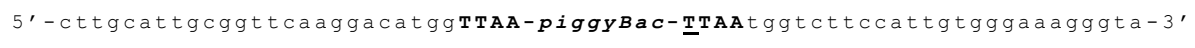 & $2 R: 23868999$ \\
\hline & & 007.8 & 5'-aatgcatgcatttcacaacaggctTTAA-piggYBac-TTAAagttgagcaatagatcgccagccat-3' & $3 \mathrm{~L}: 16708553$ \\
\hline \multirow{4}{*}{$\begin{array}{l}1 \\
\text { - } \\
\text { U } \\
z\end{array}$} & \multirow{4}{*}{$\begin{array}{c}\text { Dm- } \\
\text { 'hyPBase }\end{array}$} & 017.1 & $5^{\prime}$-ataggtacctgtcattcaataacaTTAA-piggyBac-TTAAagctttgcaaaaagtagctacatt-3' & $2 R: 11639211$ \\
\hline & & 017.2 & 5'-aataggaccgaccaccgggtattctTTAA-piggyBac-TTAAatattgtttacgttgcacttataac-3' & $3 R: 9592260$ \\
\hline & & 017.5 & 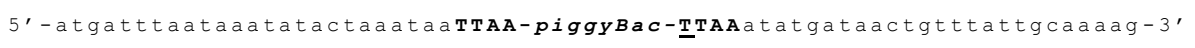 & $2 R: 8566663$ \\
\hline & & 017.7 & $5^{\prime}-$ aattctcacacttcttctttcaattTTAA-piggyBac- TTAAaagggggcattgtaaattaaaa- ${ }^{\prime}$ & $4: 1156850$ \\
\hline
\end{tabular}

chromosomal transposition could be detected. 
Supplementary Table 1. Primers used in this study

\begin{tabular}{|c|c|}
\hline Primer name & Sequence \\
\hline AP70AgeIF & AGCATACCGGTCAGGAATAAGGTTGGCTGCTGC \\
\hline AP70NheIIR & AGCATGCTAGCTTTTACACCGACATTCAAGCTAATCGGC \\
\hline B2Tub-F & AATAACCTAGGACCGGTCATTGTAGGAGCCAGAGCCAATG \\
\hline B2Tub-R & CTAATCTAGACATTTTGCTAGCAAAGTTAGGGCCCCTCTTTCAC \\
\hline co109 & ATTCGAATGGCCATGGGACG \\
\hline $\operatorname{co1} 10$ & 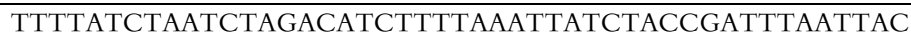 \\
\hline Hsp68_F & CGTTTCATATATAAGCGCGGTCTCGCGGCGCGTTGTC \\
\hline IC102 & GCGGAGACTCTAGCGGAAGTACACTCTTCATGGCGATA \\
\hline IC83 & GCCTTTGCTCACCATGGTGTCTCTGGATTAGACGACT \\
\hline IC86 & ATGGTGAGCAAAGGCGAAGAAG \\
\hline IC91 & CTTCACAAAGATCCTCTAGATTATTTATACAGTTCAT CCATGCCG \\
\hline IF_Cherry_SV40_F & TCGAATTCCAAAATGGTGAGCAAGGGCGAGGA \\
\hline IF_Cherry_SV40_R & CGAGATCTAGGCGCGCCGGCCAGATCGATCCAGAC \\
\hline IF_TRE_hs43_KS_R & CATTTTGGAATTCGATATCAAGCTTATCGATGGATTG \\
\hline IF_TRE_hs43_F & GCCGGCCTTGGCGCGCCAAG \\
\hline iPB-7_CtoT_F & GTTATCAAAGCCTGTGCACGGTAGTTGTCG \\
\hline iPB-7_CtoT_R & CGACAACTACCGTGCACAGGCTTTGATAAC \\
\hline piggyBac-NcoiFor & ATATCCATGGGTAGTTCTTTAGACGATGAGCATATC \\
\hline piggyBac-NotIrev & TCGAGCGGCCGCTCATCAGAAACAACTTTGGCACATATCA \\
\hline PK124_FseIGAP43Fw & GTGACTGGCCGGCCATGACGTCAATGGGAGGGCAATG \\
\hline PK124_FseIGAP43Fw & GTCAGTGGCGCGCCCTACTTGTACAGCTCGTCCATGC \\
\hline QUAS_F & GATCGGCCGGCCTAGGCTAGCGCAAAGCTTGGCTGCATC \\
\hline QUAS_R & CTTATATATGAAACGGCTCGAGCAATTCGATATCAAG \\
\hline tGFP_R & GTACGGCGCGCCTAGCTTTATTCTTCACCGGCATCTG \\
\hline
\end{tabular}




\subsection{Improvement and Use of CRISPR/Cas9 to Engineer a Sperm-marking Strain for the Invasive Fruit Pest Drosophila suzukii}

In this chapter improvement in CRISPR/Cas9 genome editing system of the invasive pest is described. It gives details on isoaltion and use of endogenous promoters of Ds heat shock protein 70 and the small nuclear RNA genes $U 6$ to drive the expressoin of Cas 9 and guide RNAs for genome engineering and provide comparison and improvement in CRISPR/Cas9 mediated homolgoy directed repair (HDR). In addition, the generation of the first D. Suzukii embryonic driver line is described which can be used to develop conditional embryonic or conditional female specific embryonic lethality as a first step to etablish the sterile insect technique (SIT) for this onerous fruit pest. Furthemore, the isolation, characterization of $D$. suzukii $\beta$-2-tubulingene $(\beta 2 t)$ and the use of its promoter to generate the first sperm-marking strains for this pest. This strain is another addition towards the establishment of the SIT for $D$. Suzukii. This work is considered the first record of using $D$. suzukii own regulatroy elemets to drive the expression of genes from an episome or as trangene and present an important contribution to the development of biotechnological pest control strategies.

Hassan M. M. Ahmed, Luisa Hildebrand, \& Ernst A. Wimmer

Authors contributions

Hassan M. M. Ahmed:

Luisa Hildebrand:

Status of manuscript:

DOI: $\underline{10.1186 / s 12896-019-0588-5}$
All experiments besides the ones performed by LH. Writing of first draft of manuscript and composition of all figures.

Testes whole mount in situ hybridizations.

Published in BMC Biotechnology.2019 


\title{
Improvement and Use of CRISPR/Cas9 to Engineer a Sperm-marking Strain for the Invasive Fruit Pest Drosophila suzukii
}

\author{
Hassan M. M. Ahmed ${ }^{1,2}$, Luisa Hildebrand ${ }^{1}$, \& Ernst A. Wimmer ${ }^{1^{*}}$ \\ ${ }^{1}$ Department of Developmental Biology, Johann-Friedrich-Blumenbach-Institute of Zoology and Anthropology, \\ Göttingen Center for Molecular Biosciences, Georg-August-University Göttingen, 37077 Göttingen, Germany. \\ ${ }^{2}$ Department of Crop Protection, Faculty of Agriculture-University of Khartoum P.O. Box 32, Postal Code 13314 \\ Khartoum North, Khartoum, Sudan \\ *Corresponding Author: E. A. Wimmer. Tel. +495513928666 \\ E-Mail address: ewimmer@gwdg.de.
}

\begin{abstract}
Background: The invasive fruit pest Drosophila suzukii was reported for the first time in Europe and the USA in 2008 and has spread since then. The adoption of type II clustered regularly interspaced short palindromic repeats (CRISPR)/CRISPR-associated (Cas) as a tool for genome manipulation provides new ways to develop novel biotechnologically-based pest control approaches. Stage or tissue-specifically expressed genes are of particular importance in the field of insect biotechnology. The enhancer/promoter of the spermatogenesis-specific beta-2-tubulin $(\beta 2 t)$ gene was used to drive the expression of fluorescent proteins or effector molecules in testes of agricultural pests and diseases vectors for sexing, monitoring, and reproductive biology studies. Here, we demonstrate an improvement to CRISPR/Cas-based genome editing in D. suzukii and establish a sperm-marking system.
\end{abstract}

Results: To improve genome editing, we isolated and tested the D. suzukii endogenous promoters of the small nuclear RNA gene $U 6$ to drive the expression of a guide RNA and the Ds heat shock protein 70 promoter to express Cas9. For comparison, we used recombinant Cas9 protein and in vitro transcribed gRNA as a preformed ribonucleoprotein. We demonstrate the homology-dependent repair (HDR)-based genome editing efficiency by applying a previously established transgenic line that expresses DsRed ubiquitously as a target platform. In addition, we isolated the $D s \_\beta 2 t$ gene and used its promoter to drive the expression of a red fluorescence protein in the sperm. A transgenic sperm-marking strain was then established by the improved HDR-based genome editing.

Conclusion: The deployment of the endogenous promoters of the D. suzukii U6 and hsp70 genes to drive the expression of $g R N A$ and Cas 9 , respectively, enabled the effective application of helper plasmid co-injections instead of preformed ribonucleoproteins used in previous reports for HDR-based genome editing. The sperm-marking system should help to monitor the success of pest control campaigns in the context of the Sterile Insect Technique and provides a tool for basic research in reproductive biology of this invasive pest. 
Furthermore, the promoter of the $\beta 2 t$ gene can be used in developing novel transgenic pest control approaches. The CRISPR/Cas9 system can be used as an additional tool for the modification of previously established transgenes.

Keywords: Cherry vinegar fly, insect transgenesis, molecular entomology, pest management, Spotted Wing Drosophila,

\section{Background}

Native to East Asia (1), the cherry vinegar fly D. suzukii, also known as the Spotted Wing Drosophila "SWD" was reported for the first time in Europe, Spain, and Italy, and the mainland USA in California in 2008 (1-3). The pest has since then expanded its geographic distribution to include all of Europe as reported by the European Plant Protection Organization (2). In the USA, the situation is as severe as in Europe. Four years after its first invasion in California, the SWD has been reported in more than 41 states (4). By now, this invasive insect pest has also been reported further down in South America: for the first time between the years 2012 and 2013 in Brazil (5) and more recently also in Argentina in four localities (6).

The devastating fruit pest $D$. suzukii infests mainly soft-skinned as well as stone fruits with a wide host range spanning cultivated and wild plants (7). In contrast to other Drosophila spp., the SWD is armoured with a sharp serrated ovipositor, which allows it to infest ripening and not only overripe or rotten fruits (8). Earlier studies have shown that economic impact due to the infestation is in the order of millions of US dollar $(9,10)$. Current control efforts mainly rely on heavy application of insecticides $(11,12)$, which is on the one hand not compatible with organic farming and prone to rapid emergence of insecticide resistance owning to the short generation time of this fly. And on the other hand, it is not safe, as the time between onset of infestation and harvest is very short and does not allow for a sufficiently long period post pesticide application. Other control strategies include the use of natural enemies such as parasitoids, predators, or pathogens (13), netting to cover the plants (14), and good cultural practices to minimise the source of infestation (15). The sterile Insect technique (SIT) presents itself as an additional safe and effective pest management strategy. It provides a species-specific, environmentally sound pest control approach (16) and is compatible with other pest control strategies in integrated pest management (IPM) programs. The system has been proposed more than half a century ago and was used to successfully eradicate the tsetse fly from Zanzibar as well as the screw worm from Libya and the USA $(17,18)$. It encompasses mass production of the target insect, removal of the females, and sterilization of males by ionizing radiation prior to release (16). Using transposon-based germline transformation, many transgenic strategies have been developed to overcome some of the drawbacks of classical SIT. A transgene-based embryonic lethality system was developed for several dipterans including the model $D$. melanogaster and the cosmopolitan fruit pest Ceratitis capitata $(19,20)$. The system relies on 
the ectopic expression of a pro-apoptotic gene during early embryonic stages, which leads to cell death and hence reproductive sterility (19). The same system has also been used for sexing, when the embryonic lethality was rendered female-specific by making use of the sexspecifically spliced intron of the transformer gene, which allows for elimination of females at the embryonic stage (20-22). Furthermore, for monitoring the competitiveness of released males, sperm-marking systems were developed for a number of pest insects and diseases vectors by driving the expression of fluorescent protein during spermatogenesis (23-26).

Recently, a revolution in genome engineering was started by the application of the CRISPR/Cas system, which stands for type II clustered regularly interspaced short palindromic repeats CRISPR/CRISPR-associated. Respective sequences were first observed in bacterial genomes in 1987 (27). Two decades later, researchers found an association between these repeated sequences and resistance of bacteria to bacteriophages (28) and showed that the bacteria use this system as an adaptive defence mechanism against invading DNA elements (29). The system consists of the Cas9 effector endonuclease, the CRISPR RNA ( $\operatorname{cr} R N A$ ), which confers specificity to Cas9, and the transactivating crRNA (tracrR $N A$ ), which facilitates maturation of $c r R N A s$ and the interaction with Cas9 protein for forming active RNP complexes $(30,31)$. The $c r R N A$ and tracrR $N A$ were fused together to generate a single chimeric gRNA that facilitated the use of the system (32). The Cas9 endonuclease can easily be programmed to target and induce DNA double strands break (DSB) by replacing the 20 nucleotides (spacer) at the 5' of the crRNA with 17-20 nucleotides (nt) complementary to the target of interest. The prerequisite for the RNP complex to unwind, bind, and induce DSB in the target DNA is a proto-spacer adjacent motif (PAM) immediately downstream of the $20 \mathrm{nt}$ target sequence, which is NGG in the case of the most commonly used Sp_Cas 9 from Streptococcus pyogenes (31). Similar to other programmable endonucleases such as Zinc finger nucleases (ZFNs) and Transcription activators like nucleases (TALENs), the role of Cas9 as a genome editing tool ends with the induction of a DSB. Repairing the genome - by either homology directed repair (HDR) or by non-homologous end joining (NHEJ) - is a function of the cell own DSB repair machinery, the stage of the cell at which the DSB is induced, and the availability of homologous DNA (32). The system has rapidly been adopted as a genome engineering tool for many model and non-model organisms including zebrafish (33), mouse (34,35), Drosophila (36), mosquitoes $(37,38)$, and human cell lines. The CRISPR/Cas9 system has also been used to induce chromosomal translocations in embryonic stem cells (39), and to engineer new balancer chromosomes in the nematode model Caenorhabditis elegans (40).

In the genetics power horse D. melanogaster, CRISPR/Cas9 has been used and delivered in different forms: as helper plasmids, mRNA and gRNA, as well as a ribonucleoprotein complex. Several promoters have been used to drive the expression of Cas 9 including germline-specific promoters of genes such as nanos and vasa, inducible promoters such as heat 
shock protein 70 ( $h s p 70$, and promoters of ubiquitously expressed genes such as Actin $5 C$. Systematic analysis of the three different promoters of the small nuclear RNA (U6) genes in $D$. melanogaster has shown that the $U 6: 3$ promoter drives the strongest expression measured by gene editing events $(41,42)$.

In Drosophila suzukii, the CRISPR/Cas9 system has been used albeit with low efficiency to mutate the genes white $(w)$ and $\operatorname{Sex}$ lethal $\left(S_{X}\right)$ using $D$. melanogaster promoters to drive the expression of $g R N A$ and $\operatorname{Cas} 9$ (43). Another study reported on the use of pre-assembled a ribonucleoprotein complex (RNP) to induce mutations in the white gene (44). The introduction of the mutations was in both studies based on NHEJ. The system has also been used to engineer by HDR a temperature sensitive mutation in the Ds_transformer-2 gene (Ds_tra-2) that leads to sex conversion. In this study a RNP complex in combination with RNA interference against the Ds_lig4 gene was used and an HDR frequency of 7.3\% was reported (45). Furthermore, a RNP complex has also been used in a behavioural study of $D$. suzukii to knockout the gene that encodes the odorant receptor co-receptor (Orco) by HDRmediated mutagenesis (46).

In applied insect biotechnology, CRISPR/Cas9 has become very popular particularly in the development of insect control strategies. One possible application for the system in SIT is the development of a reproductive sterility system that targets Cas9 to induce many DSBs at defined loci during spermatogenesis. This could mimic the desired effect of ionizing radiation in generating redundant sterility and at the same time overcome the random action of radiation affecting all organs, which reduces the overall fitness of the sterile males (47).

To restrict Cas9 activity to spermatogenesis, the isolation of a tissue-specific promoter is essential. The Drosophila $\beta 2$ tgene has been shown to code for a $\beta$-tubulin, which is expressed in a tissue-specific manner during spermatogenesis (48). Its testes-specific expression makes it a good candidate for developmental studies related to reproductive biology and male germline development as well as pest control strategies. $D m \_\beta 2 t$ is a TATA-less gene, which relies on an initiator element (Inr) as a core promoter with the testes-specific expression conferred by a $14 \mathrm{bp}$ activator element called $\beta 2$ Upstream Element 1 ( $\beta 2 U E 1)$ (49). Further elements required for the expression level are $\beta 2 U E 2$ at position -25 and $\beta 2 D E 1$ at position +60 (50). Homologs of $D m \_\beta 2 t$ were identified in a number of insects including Anopheles stephensi, Aedes aegypti, Ceratitis capitata, Anstrepha suspensa, Anastrepha ludens, and Bacterocera dorsalis (23-26). The upstream regulatory sequence has been used to drive the expression of fluorescent protein in the testes, which serves as a strategy for sex separation as well as for monitoring released males in SIT. In the major malaria vector Anopheles gambiae, the promoter of the $\beta 2 t$ gene was used to drive the expression of the homing endonuclease $I-P p o l$ during spermatogenesis. I-Ppol is a highly specific Homing Endonuclease Gene (HEG), which targets and cuts a conserved sequence within the $r D N \bar{A}$ on the $\mathrm{X}$ chromosome and 
thereby leads to $\mathrm{X}$-chromosome shredding leaving mostly $\mathrm{Y}$-chromosome bearing sperm functional, which results in sex-ratio distortion (51).

In this study, we present an improved CRISPR/Cas9-based genome engineering system for the invasive fruit pest $D$. suzukii and its application to edit a transgenic line generated using piggyBac germline transformation. Moreover, we report on the use of this editing system to generate a $D$. suzukii sperm marking line based on the $D s \_\beta 2 t$ promoter driving the expression of DsRed in the testes.

\section{Results}

\section{Improvement on CRISPR/Cas9 genome editing in Drosophila suzukii}

In order to improve on the HDR-mediated genome editing based on CRISPR/Cas9induced DSBs, we isolated endogenous polymerase II (hsp70 gene) and polymerase III promoters (U6 genes) from $D$. suzukii to drive Cas 9 or $g R N A s$, respectively. Searching for homologs of the $D$. melanogaster heat shock protein 70 ( hsp 70 ) gene, we identified the $D$. suzukii Ds_hsp70 gene, cloned and sequenced 500bp upstream of the ATG translation start codon and used this upstream sequence to drive the expression of Cas 9 .

First attempts using PCR to isolate the $U 6$ genes based on D. suzukii genome database sequences were not successful. The presence of three tandem copies obviously rendered the assembly inaccurate. Since $D$. suzukii is a close relative to $D$. melanogaster, we then tried to isolate the $U 6$ locus based on synteny cloning: we amplified and sequenced a $3.7 \mathrm{kbp}$ fragment encompassing the U6 locus. We identified three $U 6$ genes and refer to them in 5' to 3' direction as $U 6 a, U 6 b$, and $U 6 c$ (Figure 1A) to distinguish them from their D. melanogaster equivalents.

To test the efficiency of the endogenous $h s p 70$ and $U 6$ promoters in order to drive the expression of Cas 9 and $g R N A$, respectively, for mediating HDR-based genome editing, we used the embryonic line 06_F5M2 generated by piggyBac germline transformation as a target platform (Figure 1B). This driver line can be used to express the heterologous tetracyclinecontrolled transactivator $t T A$ gene specifically at early embryonic stages due to the use of the enhancer/promoter element of the cellularization gene Ds_sry $\alpha$. Such lines can be employed to establish conditional embryonic lethality for reproductive sterility $(19,20)$ or conditional female-specific embryonic lethality $(21,22,52)$. As a transgenic marker, this line expresses $D s R e d$ under the $D$. melanogaster promoter of the polyubiquitin $(P U b)$ gene. Based on a T7EndoI assay, a functional guide targeting upstream of the DsRed translation start codon was identified (Figure 1B). In a first attempt, in which donor (HMMA134), Cas9 (HMMA 056), and gRNA (HMMA104; U6c) plasmids were injected at concentrations of 350, 400, and 150 ng/ $\mu$ l, respectively, we obtained $9.5 \%$ homology directed repair (HDR) knock-in events, which we scored based on the change of the body marker from DsRed to EGFP(Figure 1C- 
E). Sequencing of the knock-in junctions revealed faithful scar-less HDR events. The HDR was facilitated by the 1989bp left homology arm (PUb promoter) and the 672bp right homology arm (DsRed).

To compare the three promoters of the $D s U 6$ genes, we injected in a second attempt donor (HMMA134), Cas9(HMMA056), and either of the three gRNA plasmids HMMA102 (U6a), HMMA103 (U6b), or HMMA104 $(U 6 c)$ at a concentration of 400, 400 and $250 \mathrm{ng} / \mu \mathrm{l}$, respectively. This resulted in HDR events of $12.5 \%, 2 \%$, and $15.5 \%$ for $U 6 a, U 6 b$, and $U 6 c$, respectively (Figure 1F). Injection of a RNP complex resulted in 33\% HDR events (Figure $1 \mathrm{~F})$. This indicates, that at slightly higher concentrations of donor template and gRNA plasmids, we were able to obtain $15.5 \%$ knock-in events using the $U 6 c$ promoter. The $U 6 b$ showed the lowest performance with only $2 \%$ knock-in events, and U6a was intermediate with $12.5 \%$ efficiency (Figure 1F). Interestingly, the tendency observed for the strength of the different promoters is in line with their D. melanogaster counterparts. The high HDR-rates of above $10 \%$ indicate that the use of the endogenous promoters allows for effective application of helper plamids instead of RNPs to induce HDR-dependent knock-ins, which represents an improvement for CRIPR/Cas9-based genome editing in D. suzukii.

\section{Isolation of the 82 tubulin gene from Drosophila suzukii}

To be able to drive sperm-specific gene expression, we identified the $D s \_\beta 2 t$ gene by homology search in the $D$. suzukiigenome database (www.spottedwingflybase.org) using the $D m \_\beta 2 t$ sequence as query. The open reading frame of the $D s \_\beta 2 t$ gene from the translation start codon to the stop codon is $1341 \mathrm{bp}$, which is interrupted by a $215 \mathrm{bp}$ intron. The gene has a 5'UTR of $196 \mathrm{bp}$, which demarcates the transcription start site (Figure 2A). Conceptual translation of the $D s \_\beta 2 t$ coding sequence gives rise to a protein of 446 amino acids.

To validate the testes-specific gene expression of the isolated $D s \_\beta 2 t$ gene, we performed whole mount in situ hybridization on the complete reproductive tract of 3-5 days old males using DIG-labelled antisense and sense RNA probes against the $D s \_\beta 2 t 5$ 'UTR and exon I. These in situ hybridizations detected expression only in the testes with no expression at the apical part that consists of stem cells (Figure 2B). No transcription was detected in the rest of the reproductive tract (Figure 2B) or with sense RNA probe as negative control (Figure 2C).

\section{Generation of a sperm-marking line of Drosophila suzukii}

To identify the necessary upstream and downstream regulatory elements driving spermspecific gene expression, we compared the $D$. suzukii $\beta 2 t$ sequence with the characterized counterpart in $D$. melanogster. The $14 \mathrm{bp}$ upstream activator element $\beta 2 t U E 1$ that confers testes specificity to the $\beta 2 t$ gene was found at the exact position -51 to -38 relative to the transcription start site with a $C>G$ exchange at position -41 and a $T>A$ exchange at position -39 (Figure 3A). A second upstream regulatory element, $\beta 2 t U E 2$, which is not involved in 
specificity but its overall activity, was identified at position -32 to -25 with a $G>T$ exchange at position -32 and an $\mathrm{A}>\mathrm{C}$ exchange at position -28 . Another element that functions as a TATAAA-box in TATA-less promoter is the $7 \mathrm{bp}$ initiator sequence encompassing the transcription start, which was identified -3 to +4 with the first and last nucleotide differing from $D$. melanogaster (Figure 3A). A further element involved in $\beta 2 t$ promoter function is the $\beta 2 t D E 1$ element that is highly conserved and lies relative to the transcription start site at position +51 to +68 (Figure $3 \mathrm{~A}$ ).

To examine whether the 51 bp upstream regulatory element plus 196 bp 5'UTR ( -51 to +196 ) drives strong testes-specific gene expression, we fused this $247 \mathrm{bp}$ enhancer/promoter fragment of the Ds_ $\beta 2 t$ gene to DsRed.T3 (Figure 3B) and performed an HDR-based knock-in into the $D$. suzukii embryonic piggyBac line 06_F5M2, which we had used before as target platform (Figure 3B). The repair template consisted in this case of EGFP fused to the PUb promoter followed by $S V 40$ 3'UTR and the 247 bp $D s \_\beta 2 t$ promoter fused to DsRed.T3 (Figure 3B). The HDR-based knock-in resulted with 13.3\% efficiency. One of the resulting D. suzukii lines, 134M16M2, showing a ubiquitous green fluorescence and testes-specific red fluorescence (Figure 3C-H), was moleculary characterized to confirm the proper HDR event. In this line, red fluorescent sperm could be detected in the testes (Figure 3I-L) and males of this line transferred red fluorescent sperm to the female spermatheca (Figure $3 \mathrm{M}-\mathrm{P}$ ). This line 134M16M2 thus serves as a sperm-marking line for this invasive pest insect.

\section{Discussion}

The programmable genome editing system CRISPR/Cas9 has enabled a series of new strategies of biotechnological engineering in model and non-model organisms. Based on the objective of the study, financial resources, and availability of functional promoters, researchers can choose the best strategy for delivery of CRISPR/Cas9 components. From published literature, it can be concluded that the most efficient strategy is germline-specific transgenic expression of Cas9, followed by application of RNP-complexes, then mRNA and gRNA co-injection, and with the least efficiency helper plasmids co-injection $(42,53)$. The latter, however, is the most convenient even though it requires the identification and characterization of suitable promoters.

CRISPR/Cas9 holds big promises in the field of insect biotechnology especially for the development of novel pest control strategies, such as reproductive sterility systems based on chromosome shredding (47). To be able to engineer such strategies in D. suzukii, promoters that drive strong expression of gRNAs and other components are of particular importance. Inducible promoters of heat shock genes such as D. melanogaster hsp70 and Tribolium 
castaneum Tc_hsp68 have been used for a long time to conditionally express genes both transiently from a plasmid and as transgenes $(54,55)$.

Due to their defined transcription start site and transcription termination, the RNA polIII promoters of the small nuclear RNA genes (snRNA) U6 have been widely used to express short hairpins to induce an RNA interference effect. With the development of the CRISPR/Cas9 genome editing system, such promoters gained even more popularity and have intensively been used to drive the expression of the chimeric gRNAs transiently and as transgene components from mammals to plants. D. melanogaster has three copies in tandem on the right arm of chromosome 3 and have the cytological map location 96A, based on which they were termed $U 6: 96 \mathrm{Aa}, U 6: 96 \mathrm{Ab}$, and $U 6: 96 \mathrm{Ac}$. The promoters of the three genes were systematically tested and the promoter of the U6:96Ac gene (referred to also as U6:3) outperforms the other two, which made it the promoter of choice among Drosophilists. Our results are consistent in this respect, as also the Ds_U6c promoter has the highest effectivity (Figure 1F).

Previous reports demonstrated the functionality of the promoters of $D m-U 6: 3$ and vasa genes to drive expression of $g R N A$ and Cas 9 , respectively, to target and mutate $D$. suzukii $W$ and $S_{x} l$ by NHEJ but with low frequency. The authors argued that this low efficiency might be attributed to the use of plasmids to drive the expression of Cas 9 and $g R N A$ or their bulk crossing scheme (43). Another study demonstrated the feasibility of using RNP-complexes to induce mutations in D. suzukii $w$ by NHEJ (44). In a more recent study, researchers used RNP-complexes to induce DSBs and were able to knock-in by HDR a mutated temperature-sensitive version of Ds-tra2 along with a transformation marker cassette. They reported on 7.3\% HDR events even though they tried to shift the cell DSB repair machinery towards HDR by co-injection of dsRNA against the Ds_lig4 gene (45). In our hands, using RNP complex resulted in a four times higher rate of HDR-based knock-ins. However, no direct comparison with the previous studies is possible since the target itself is different. Anyway, also our helper plasmid co-injections yielded a two times higher rate of HDR-based knock-ins, which indicates that the isolated endogenous promoters allow for an efficient application of the CRISPR/Cas system with the more convenient use of plasmid helpers. However, if the objective is to manipulate the genome and recombinant Cas9 is available, the RNP approach is probably the best option, if no transgenic lines expressing Cas 9 in the germline are available. Studies in D. melanogaster and mosquitoes also showed that the use of RNP-complexes always leads to better editing results compared to injection of plasmids or mRNA and in vitro transcribed gRNA.

The use of the regulatory elements (enhancer/promoter) of sex-, tissue-, or stage-specifically expressed genes to drive effector molecules in a particular sex or developmental stage is not only useful in basic research to elucidate gene function, but also in applied insect 
biotechnology to develop transgene-based pest control strategies. The gene $\beta 2 t$ has been identified in a number of insects to be testes-specific with its activity starting at the late larval instar. The gene in D. melanogaster is known to code for a 446aa protein. Here, we identified the $D$. suzukii homolog that shows at the amino acid level $100 \%$ identity but not at the nucleotide level. Interestingly, the transcript structure of the $D s \_\beta 2 t$ gene revealed the presence of a $215 \mathrm{bp}$ intron (Figure 2A) compared to a highly conserved intron of $57 \mathrm{bp}$ in Aedes egypti (24), 58 bp in Anastrepha ludens, 59 bp in D. melanogaster, 60 bp Anstrepha suspensa, and $67 \mathrm{bp}$ in Bacterocera dorsalis (25). Testes whole mount in situ hybridization identified a similar expression pattern as previously obtained in $D$. melanogasterwith the apical part of the testes that contains the stem cells not expressing the gene. The testes specificity of the gene is conferred by a $14 \mathrm{bp}$ activator element upstream of the transcription start site called upstream element $1 \beta 2 t U E 1$, which is not only contextually conserved but also spatially relative to the transcription start site and other regulatory elements. This activator element was also identified in $D$. suzukii, which shares high similarity to its $D m \_\beta 2 t$ counterpart. The other elements that are quantitatively contributing to the expression of $\beta 2 t$ were also identified in exactly the same positions as in $D$. melanogaster relative to each other and to the transcription start site.

The promoter of the $\beta 2 t$ gene has been used to drive the expression of a fluorescent protein in mosquitoes and tephritid fruit flies $(23,24,26)$, which serve as a sexing system to automate separation of males from females and also as a monitoring system for released males in the context of SIT programs. The generated sperm marking strain of D. suzukii proved that the $247 \mathrm{bp}$ regulatory sequence made of $51 \mathrm{bp}$ upstream sequence plus $196 \mathrm{bp}$ leader immediately upstream of the translation start codon has the necessary elements to drive expression of effector molecules specifically in the sperm. The fluorescent sperm can also be identified stored in the spermathecae of wild type females mated to the transgenic sperm marked strain, which facilitates monitoring and allows assessment of the competitiveness of released sterile males compared to their wild type counterparts. The sperm marking system can also help in conducting reproductive biology studies that will enrich our understanding of the biology of this pest and allow us to better design pest control strategies. For example, the promoter of the $\beta 2$ tgene in Anopheles was used to drive the expression of an HEG that targets and shreds the $\mathrm{X}$ chromosome in the mosquito during spermatogenesis leading towards a $\mathrm{Y}$ sperm bias and as a consequence to sex ratio distortion, which eventually can lead to a population collapse (51).

\section{Conclusion}

We obtained improved usability of the CRISPR/Cas9 gene editing in D. suzukii compared to previous reports (43-45) by the employment of helper plasmids that contain endogenous promoters of the $U 6$ and $h s p 70$ genes to drive the expression of $g R N A$ and $C a s 9$, respectively. 
Moreover, we show that the CRISPR/Cas9 system can be used as an additional tool for the modification of previously established transgenes. The identification and cloning of the $\beta 2 t$ promoter enabled us to generate a sperm-marking system in D. suzukii, which provides a tool for basic research in reproductive biology and should help to monitor the success of pest control campaigns in the context of SIT (23-26). In addition, the $\beta 2 t$ promoter can be used in developing novel transgenic pest control approaches (47) for this invasive pest insect.

\section{Methods}

Unless otherwise specified, all PCR amplifications were performed using Phusion DNA polymerase and Phusion-HF buffer (New England Biolabs GmbH, D-65926 Frankfurt am Main). Routine plasmid min-preps and PCR products were purified using NucleoSpin ${ }^{\circledR}$ Plasmid and NucleoSpin ${ }^{\circledR}$ Gel and PCR Clean-up kits (Macherey-Nagel GmbH \& Co., 52355 Dueren, Germany), respectively. Plasmid vectors for microinjections were prepared using NucleoSpin ${ }^{\circledR}$ Plasmid Transfection-grade (Macherey-Nagel) or QIAGEN Plasmid Plus Midi Kit (QIAGEN GmbH, 40724 Hilden, Germany). Primers used are listed in Additional File 1: Supplementary Table 1.

\section{Fly strain and husbandry}

All fly experiments were performed in our well-equipped safety level one (S1) laboratory, which is certified for generating and using genetically modified insects. Wild type $D$. suzukii from Italy (kindly provided by Prof. Marc F. Schetelig) as well as generated transgenic lines were reared on standard Drosophila food supplemented with baker yeast and kept at $25^{\circ} \mathrm{C}$ throughout this study. For germline transformation, flies were transferred to Drosophila egg laying cages and allowed to lay eggs on apple juice agar plates with some yeast on top to increase egg laying.

\section{Nucleic acid isolation}

Genomic DNA was isolated from a mix of adult males and females of D. suzukii (Italian strain) using NucleoSpin ${ }^{\circledR}$ DNA Insect (Macherey-Nagel) according to the manufacturer instructions. To generate a testes-specific cDNA library, testes of 100 males (3-4 days old) were dissected in ice cold $1 \mathrm{X}$ PBS and used for total RNA preparation using ZR Tissue \& Insect RNA MicroPrep (Zymo Research Europe, 79110 Freiburg) according to manufacturer instructions.

\section{Isolation of DsU6 and hsp 70 genes}

Based on synteny we identified $D$. suzukii the homologs of D. melanogastergenes Esyt2 and REPTOR bordering the U6locus. Primer pair HM\#137/138 was designed on the conserved 
parts of these genes and used to PCR amplify the sequence between them supposedly containing the Ds_U6 locus, (initial denaturation temperature $98^{\circ} \mathrm{C} 3 \mathrm{~min}$ followed by 35 cycles of $98^{\circ} \mathrm{C} 30 \mathrm{sec}, 72^{\circ} \mathrm{C} 2 \mathrm{~min} 30 \mathrm{sec}$ ). A $3.7 \mathrm{kbp}$ fragment was obtained and sequenced.

To identify the D. suzukii heat shock protein 70 (Dshsp70) gene, we BLASTed D. melanogaster hsp70Aa in the D. suzukii genome data base (www.spottedwingflybase.org) and compared the amino acid sequence as well as the corresponding DNA sequence individually to their D. melanogaster counterparts using the geneious program version 10.2.6 (Auckland, 1010, New Zealand).

\section{Isolation of $D s \beta 2 t$ gene and its 5'UTR}

To isolate the spermatogenesis specific beta-2-tubulin ( $\beta 2 t)$ gene of $D$. suzukii, we searched in the www.spottedwingflybase.org with the $D$. melanogaster $D m \_\beta 2 t$ gene. A putative $D s$ $\beta 2 t$ gene sharing high homology to $D m \_\beta 2 t$ was PCR amplified from genomic DNA using primer pair $\mathrm{HM \# 25/26}$ and the PCR program $98^{\circ} \mathrm{C}$ for $3 \mathrm{~min}$ followed by 35 cycles of $98^{\circ} \mathrm{C}$ $30 \mathrm{sec}, 72^{\circ} \mathrm{C} 1 \mathrm{~min} 40 \mathrm{sec}$, and $7 \mathrm{~min}$ final elongation at $72^{\circ} \mathrm{C}$. The amplified fragment was purified, blunt cloned into pJet1.2 vector (Thermo Fisher Scientific, 64293 Darmstadt, Germany), and sequenced using standard primers pJet1.2_fwd and pJet1.2_rev.

Since the 5'UTR of $\beta 2 t$ has some regulatory elements, whose position relative to the transcription start site and the upstream regulatory elements is highly conserved and important for correct tissue specific expression, it was imperative to isolate the 5'UTR and to identify the transcription start site. To do so, $1.7 \mu \mathrm{g}$ of testes total RNA were used to generate a 5' RACE-ready cDNA library using the SMAR Ter ${ }^{\mathrm{TM}}$ RACE cDNA amplification kit (Takara Bio Europe SAS, 78100 Saint-Germain-en-Laye, France) according to manufacturer instructions. The 5'UTR was recovered by RACE PCR using gene specific primer HM\#33 and universal primer (UPM) provided with the kit using Advantage2 DNA polymerase (Takara) with the following program: $94^{\circ} \mathrm{C} 2 \mathrm{~min},\left(94^{\circ} \mathrm{C} 30 \mathrm{sec}, 72^{\circ} \mathrm{C} 3 \mathrm{~min}\right) 5 \mathrm{X},\left(94^{\circ} \mathrm{C} 30\right.$ sec, $\left.70^{\circ} \mathrm{C} 30 \mathrm{sec}, 72^{\circ} \mathrm{C} 3 \mathrm{~min}\right) 5 \mathrm{X},\left(94^{\circ} \mathrm{C} 30 \mathrm{sec}, 68^{\circ} \mathrm{C} 30 \mathrm{sec}, 72^{\circ} \mathrm{C} 3 \mathrm{~min}\right) 30 \mathrm{X}$. A single prominent band was recovered, purified, cloned into pCRII (Thermo Fisher Scientific) to generate pCRII_Dsb2t_5'UTR (HMMA24), and sequenced using a standard M13 primer.

\section{Testes whole mount in situ hybridization}

To generate DIG-labelled sense and antisense RNA probes of $D s \_\beta 2 t$, we prepared DNA templates for in vitro transcription by PCR amplification of the 5'RACE-fragment including the Sp6 or T7 promoters from pCRII_Ds $\beta 2 \mathrm{t}$ 5'UTR (HMMA24). Primer pairs HM\#33/128and HM\#41/127 were used respectively with the following PCR conditions: initial denaturation at $98^{\circ} \mathrm{C} 3 \mathrm{~min}$, followed by 35 cycles of $98^{\circ} \mathrm{C} 30 \mathrm{sec}, 72^{\circ} \mathrm{C} 50 \mathrm{sec}$ with a final elongation step of $7 \mathrm{~min}$. RNA probes were synthesized using DIG-labelling kit (Thermo Fisher Scientific) according to manufacturer instructions using 200ng of DNA as 
template in a total reaction mix of $10 \mu \mathrm{l}$. The reaction was allowed to proceed for $2 \mathrm{~h}$ at $37^{\circ} \mathrm{C}$ followed by Turbo DNaseI treatment (Thermo Fisher Scientific) for $15 \mathrm{~min}$ to remove template DNA. $2 \mu \mathrm{l}$ of $0.2 \mathrm{M}$ EDTA was used to inactivate the reaction. Sense and antisense probes were precipitate and resuspended in 100 $\mu \mathrm{l}$ RNA resuspension buffer (5:3:2 H2O: 20X SSC: formaldehyde) and stored at $-80^{\circ} \mathrm{C}$.

Testes of 3-5 days old males were dissected in ice cold 1X Phosphate buffered saline (PBS) and fixed in PBF-tween (4\% formaldehyde and $0.1 \%$ tween 20 in 1X PBS) for $20 \mathrm{~min}$ at room temperature. In situ hybridization was performed according to an established protocol (56) with inclusion of dehydration steps according to Zimmerman et al. (57).

\section{Plasmid construction}

To generate plasmid HMMA006, 300 bp upstream of Ds_sryo plus 50bp 5'UTR sequence were PCR amplified using primer pair HM\#23/24 introducing AgeI/NheI cut sites respectively and cloned into AgeI/NheI cut site of KNE007 (58) upstream of tTA CDS replacing the $D m \_\beta 2 t$ promoter. Description of the $D s \_s r y \alpha$ gene and its cloning will be described elsewhere (Ahmed et al.)

To generate pSLaf_T7-BbsI-BbsI-ChiRNA_af (HMMA034) for in vitro transcription of gRNAs, annealed oligos HM\#55/56 generating T7 promoter and 2X BbsI restriction sites were cloned into BbsI/HindII digested plasmid p U6-chiRNA(Addgene: \#45946) giving rise to HMMA033. Next, the HindII/SacI T7-BbsI-BbsI-chiRNA fragment from HMMA033 was cloned into pSLaf1180af (59) HindII/ SacI cut sites.

To generate plasmids pDsU6a-BbsI-BbsI-chiRNA-DSE (HMMA091), pDsU6b-BbsIBbsI-ChiRNA DSE (HMMA092), and pDsU6c-BbsI-BbsI-chiRNA-DSE (HMMA093) for transient expression of gRNAs, primer pairs HM\#358/159, HM\#104/158, and HM\#360/160 were used to amplify the promoters of $s n R N A$ genes $U 6 a$, U6b, and U6c, respectively, with PCR condition $98^{\circ} \mathrm{C} 3$ min followed by 5 cycles of $98^{\circ} \mathrm{C} 30 \mathrm{sec}, 66^{\circ} \mathrm{C} 40$ sec, and $72^{\circ} \mathrm{C} 1 \mathrm{~min}$ then 30 cycles of $98^{\circ} \mathrm{C} 30 \mathrm{sec}, 72^{\circ} \mathrm{C} 1 \mathrm{~min} 40 \mathrm{sec}$ with a final elongation $72^{\circ} \mathrm{C}$ for $7 \mathrm{~min}$. The promoters were then cloned into HMMA034 by megaprimer PCR cloning (60) using 30ng of plasmid HMMA034 and 200ng of the promoter as megaprimer in a $25 \mu \mathrm{l}$ reaction with $\mathrm{PCR}\left(98^{\circ} \mathrm{C} 3 \mathrm{~min},\left[98^{\circ} \mathrm{C} 30 \mathrm{sec}, 72^{\circ} \mathrm{C} 2 \mathrm{~min} 30 \mathrm{sec}\right] 30 \mathrm{X}, 72^{\circ} \mathrm{C} 7 \mathrm{~min}\right)$ generating plasmids HMMA088, HMMA089, and HMMA090. Finally, 250 bp of the sequence downstream of the $U 6 c$ termination sequence was PCR amplified from genomic DNA using primer pair $\mathrm{HM \# 186/187}$ with PCR $\left(98^{\circ} \mathrm{C} 3 \mathrm{~min},\left[98^{\circ} \mathrm{C} 30 \mathrm{sec}, 68^{\circ} \mathrm{C} 30 \mathrm{sec}\right.\right.$, $72^{\circ} \mathrm{C} 20 \mathrm{sec}$ ] $35 \mathrm{X}$ with a final elongation of $7 \mathrm{~min}$ at $72^{\circ} \mathrm{C}$ ). The amplified fragment was then cloned into HMMA088, HMMA089, and HMMA090 by megaprimer cloning as described above with annealing temperature at $68^{\circ} \mathrm{C}$. 
For Cas9 recombinant protein expression, the plasmid pET-T7-3XFlag-nls-Cas9-nls6XHis Tag(HMMA101) was generated. The sumo part of the pET-SUMO expression vector was removed using XhoI/NdeI and the annealed oligos HM\#152/153 were cloned introducing $2 \mathrm{X}$ BsaIsites giving rise to HMMA080. The 4.3Kb Bbs/ XbaI 3XFlag-nls-Cas9$n l s$ fragment was excised from HMMA066 and cloned into Bsallinearized HMMA080 to give rise to HMMA099. Finally, annealed oligos HM\#180/181 introducing a 6XHisTag were cloned into FseI/BasI digested plasmid HMMA099. Plasmid HMMA066 was generated by cloning ClaI/HpaI fragment 3XFlag-nls-Cas9-nls from HMMA039 into ClaI/HpaI cut \#1215 (20) giving rise to HMMA065 followed by cloning of annealed self-complementary oligo HM\#102 into the ClaI site of HMMA065 to introduce 2X BbsI restriction sites. Cas9 protein was expressed and purified according to Paix et al. (61), and frozen at $-20^{\circ} \mathrm{C}$ until needed.

The plasmid pSLaf_Dshsp70P_Cas9-SV40_af(HMMA056) to express Cas9 transiently was generated by cloning of the $4.2 \mathrm{~Kb} \mathrm{ClaI} / \mathrm{XbaI}$ fragment containing insect codon optimized Cas $9 \mathrm{CDS}$ with $\mathrm{N}$ and $\mathrm{C}$ terminal nuclear localization signals from plasmid \#46294 (Addgene) into ClaI/XbaIdigested pCS2-Sp6-Cas9-SV40 (Addgene: \#47322) replacing the mammalian codon optimized Cas 9 CDS giving rise to HMMA039. The Ds_hsp70 promoter was PCR amplified from genomic DNA using primer pair HM\#73/75 with PCR using the following condition: $98^{\circ} \mathrm{C} 3 \mathrm{~min}\left[\left(98 \mathrm{C}{ }^{\circ} \mathrm{C} 30 \mathrm{sec}, 66^{\circ} \mathrm{C} 40 \mathrm{sec}, 72^{\circ} \mathrm{C} 1 \mathrm{~min}\right) 5 \mathrm{X},\left(98^{\circ} \mathrm{C} 30 \mathrm{sec}, 72^{\circ} \mathrm{C} 1\right.\right.$ min $40 \mathrm{sec}) 35 \mathrm{X}$ with a final elongation step of $7 \mathrm{~min}$ at $72^{\circ} \mathrm{C}$. The fragment was purified and cloned into EcoRI/ClaI cut \#1215 (20) to give rise to HMMA052. Finally, Cas9-SV4O was excised from HMMA039 by ClaI/HpaIand cloned into ClaI/HpaIcut HMMA052 generating HMMA056.

To generate donor plasmid HMMA134, a 3.2Kb fragment containing PUb-nls-EGFP-SV4O was excised from \#1254 (20) using SacI/AflIIand cloned into SacI/AflII cut pSLaf1108af(59) giving rise to plasmid HMMA094. DsRed CDS was PCR amplified from plasmid KNE007 (58) using primer pair (HM\#37/167) with PCR $\left(98^{\circ} \mathrm{C} 3\right.$ min followed by 35 cycles of $98^{\circ} \mathrm{C}$ $30 \mathrm{sec}, 72^{\circ} \mathrm{C} 1 \mathrm{~min}$ and a final elongation of $7 \mathrm{~min}$ at $\left.72^{\circ} \mathrm{C}\right)$. The fragment was phosphorylated and ligated into blunted AfIII cut HMMA095 generating HMMA096. To change the target PAM sequence in front of EGFP from TGG to TGA in the repair template (Figure 1B), PCR mutagenesis using primer pair HM\#221/222 was performed $\left(98^{\circ} \mathrm{C} 3\right.$ min followed by 30 cycles of $98^{\circ} \mathrm{C} 30 \mathrm{sec}, 72^{\circ} \mathrm{C} 4 \mathrm{~min}$ and final elongation of $7 \mathrm{~min}$ at $72^{\circ} \mathrm{C}$ ) to give rise to HMMA097, which results in changing the second amino acid of the EGFP from valine to methionine. Finally, the 247 bp $D s \_\beta 2 t$ regulatory sequence spanning -51 to +196 was PCR amplified using primer pair $\mathrm{HM \# 285/252}$ with PCR conditions $98^{\circ} \mathrm{C} 3 \mathrm{~min}\left[\left(98^{\circ} \mathrm{C} 30 \mathrm{sec}\right.\right.$, $\left.60^{\circ} \mathrm{C} 30 \mathrm{sec}, 72^{\circ} \mathrm{C} 20 \mathrm{sec}\right) 5 \mathrm{X},\left(98^{\circ} \mathrm{C} 30 \mathrm{sec}, 72^{\circ} \mathrm{C} 1 \mathrm{~min}\right) 30 \mathrm{X}$ with a final elongation step of $7 \mathrm{~min}$ at $72^{\circ} \mathrm{C}$. The promoter was then cloned upstream of DsRed in HMMA097 by megaprimer PCR cloning as described previously with annealing at $61^{\circ} \mathrm{C}$. 


\section{Guide RNAs design, cloning, and validation}

Guide RNAs were identified using the online target finder tool built by Wisconsin University (http://targetfinder.flycrispr.neuro.brown.edu/). Identified potential targets were checked against $D$. suzukii database to exclude those with off-target sites. For each potential target, two oligos, a forward and reverse, were designed and the respective overhangs were added. Oligos were ordered as normal primers without phosphorylation. The two oligos for each target were annealed at a concentration of $10 \mu \mathrm{M}$ in a total volume of $100 \mu \mathrm{l}$ in a heat block. The gRNAs were validated using a T7EndoI assay $(62,63)$. Each $g R N A$ plasmid was mixed with Cas 9 plasmid HMMA056 at a concentration of 400/500 $\mathrm{ng} / \mu \mathrm{l}$, respectively, and injected into 50 pre-blastoderm embryos. 10 - 15 hatching larvae were collected in $1.5 \mathrm{ml}$ Eppendorf tubes and crushed by using a pipette tip against the tube wall. $200 \mu$ l of squishing buffer (19) was added and mixed well. The tubes were then incubated at $55^{\circ} \mathrm{C}$ for $1 \mathrm{~h}$ with occasional vortexing. Tubes were then centrifuged, and $5 \mu \mathrm{l}$ of the supernatant was used as a template in $50 \mu \mathrm{l}$ PCR reactions using primers HM\#192/69. PCR products were gel purified, quantified, and 400ng were mixed in 1 X NEB 2.1 buffer in a total volume of $19 \mu 1$. DNA was denatured, rehybridized, $0.75 \mu \mathrm{l}$ of $\mathrm{T} 7 \mathrm{EndoI}(\mathrm{NEB})$ were added, and incubated at $37^{\circ} \mathrm{C}$ for 20 minutes. The reactions were stopped using $2 \mu \mathrm{l}$ of $0.25 \mathrm{M}$ EDTA and run in a 1.5\% agarose gel. Only one guide showed obvious digest by T7 EndoI. Wild type un-injected larvae were used as control. To generate the plasmids expressing the functional guide RNA against the identified target upstream of DsRed(Figure 1B), annealed oligos HM\#161/162 and HM\#169/162 were cloned by golden gate $(64,65)$ into gRNA vectors HMMA091, HMMA092, and HMMA093 to generate pU6a_Red1chic HMMA102, pU6b_Red1chiHMMA103, and pU6c_Red1chi HMMA104, respectively.

\section{In vitro transcription of the gRNA}

The functional gRNA was cloned by ligation of annealed oligos HM\#162/215 into BbsI cut plasmid HMMA035, which was then used to generate the template for in vitro transcription by PCR using primer pair HM\#84/128. In vitro transcription of $g R N A$ was performed using MEGAscript ${ }^{\circledR}$ (Ambion) according to the manufacturer protocol. The reaction was allowed to proceed for $2 \mathrm{~h}$ at $37^{\circ} \mathrm{C}$ followed by DNA template removal using $1 \mu \mathrm{l}$ DNase I for 30 minutes. $g R N A$ was purified using RNA clean and concentrator (Zymo Research) and the concentration was determined by nano-drop (Thermo Fisher Scientific) and stored at $-80^{\circ} \mathrm{C}$.

\section{Germline transformation}

All embryonic injections were performed using transfection grade plasmid preparations without further precipitation steps. To generate the embryonic driver line 06_F5M2 by random piggyBac integration, the transformation vector HMMA006 and the helper plasmid MK006 (58) were mixed at a final concentration of 400 and $200 \mathrm{ng} / \mu \mathrm{l}$ respectively. To validate 
that the transgene represents a single integration even, we performed inversePCR as described (58) using XhoI and EcoRI restriction enzymes. For both the 5 and 3' junctions, we each obtained only a single fragment, whose sequences confirmed a single integration site in the second intron of a gene referred to as Suppressor of Under Replication (Additional File 2: piggyBac insertion in D. suzukiiline 06_F5M2).

For the transgene editing experiments using CRISPR/Cas9, DNA was mixed at a concentration of 400, 150, and $350 \mathrm{ng} / \mu \mathrm{l}$ for Cas9 (HMMA056), gRNA (HMMA102, HMMA103, or HMMA104), and donor plasmid HMMA097, respectively. Higher concentration was used at 400, 250, and $400 \mathrm{ng} / \mu \mathrm{l}$, respectively. All DNA injection mixes were prepared in $1 \mathrm{X}$ injection buffer $\left(5 \mathrm{mM} \mathrm{KCl}, 0.1 \mathrm{mM} \mathrm{NaH}_{2} \mathrm{PO}_{4}, \mathrm{pH}\right.$ 6.8). For RNP injection, recombinant Cas9 endonuclease, gRNA, and donor plasmid HMMA097 were mixed together at a final concentration of $300 \mathrm{ng} / \mu \mathrm{l}, 150 \mathrm{ng} / \mu \mathrm{l}$, and $400 \mathrm{ng} / \mu \mathrm{l}$ respectively, incubated at $37^{\circ} \mathrm{C}$ for 10 minutes for the RNP-complex formation, and injected into 90 preblastoderm embryos.

Injection needles were prepared as previously described (58). To inject in D.suzukii embryos, the eggs have to be squeezed out of the apple agar plates individually using home-made closed-tip glass pipettes. Embryos were then de-chorionated for 3 minutes using generic Clorox (DanKlorix, CP GABA GmbH, Hamburg, Germany) containing 2.5\% sodium hypochlorite at final concentration of $1.25 \%$ sodium hypochlorite and washed in washing buffer $(100 \mathrm{mM} \mathrm{NaCl}, 0.02 \%$ Triton X-100) followed by thorough wash with desalted water. Embryos were then aligned on apple agar blocks and transferred to double sticky tape on a coverslip and covered by Voltalef $10 \mathrm{~S}$ oil (VWR International, Darmstadt, Germany). Injections were performed using a Femtojet (Eppendorf, Hamburg, Germany) and a manual micromanipulator. Excessive oil was drained and the injected embryos were incubated on apple agar plates at the room temperature until hatching. Larvae were manually transferred to fly food vials. Each emerging $G_{0}$ fly was out-crossed to 3-4 wild type individuals of the opposite sex.

\section{Microscopy}

Screening for transgenic flies and fluorescence imaging were performed using a Leica M205 FA fluorescence stereomicroscope equipped with camera $\mathrm{Q}$ imaging Micropublisher 5.0 RTV (Leica Mikrosysteme Vertrieb Gmb, Wetzlar, 35578 Germany). Transgenic flies were screened using filter sets RFP (excitation: ET546/10x, emission: ET605/70m) or GFP-LP (excitation: ET480/40, emission: ET510 LP), respectively, and imaged using cold light (Figure 1C) or filter sets: RFP (Figures 1D; 3F-H), EYFP (excitation: ET500/20, emission: ET535/30) for Figure 1E, or GFP-LP (Figure 3C-3E). 
Epifluorescence microscopy was performed using a Zeiss Imager.Z2 equipped with two cameras, Axiocam 506 mono and Axiocam 305 colour (Zeiss, 73447 Oberkochen, Germany). The testes or the spermathecae were dissected in ice-cold PBS, fixed for 10 minutes in 4\% formaldehyde prepared in $0.1 \%$ PBS-tween 20, permeabilized for 10 minutes using $1 \%$ Triton $\mathrm{X}-100$ in PBS, and nuclei were stained for 10 minutes using DAPI (4',6-Diamidino-2Phenylindole, Dihydrochloride) at a concentration of $1 \mu \mathrm{g} / \mathrm{ml}$. Samples were mounted in $70 \%$ glycerol and the spermathecae were broken open using dissection needles. The tissues were imaged under bright field and to observe cell nuclei and expression of DsRed, images were taken with filters for DAPI (excitation: 335-383, emission: 420-470) or DsRed (excitation: 533-558, emission: 570-640), and composed in ZEN Blue (Zeiss).

\section{Competing interests:}

The authors declare that they have no competing interests.

\section{Funding:}

H.M.M.A was supported by the German Academic Exchange Service (DAAD), which had no role in the design of the study, the collection, analysis, and interpretation of data, or in writing the manuscript.

\section{Authors' Contributions:}

E.A.W and H.M.M.A. conceived and designed the study; H.M.M.A. isolated the genes, designed the constructs and generated the transgenic lines; L.H. performed in situ hybridizations; E.A.W and H.M.M.A. wrote the manuscript; H.M.M.A. prepared the figures; all authors read and approved of the final manuscript.

\section{Acknowledgements:}

We would like to thank Prof. Dr. Marc F. Schetelig (Justus-Liebig-University Giessen) for providing wild type Drosophila suzukii(Italian strain) and Kolja N. Eckermann (University of Göttingen) for providing plasmid KNE007. Our thanks extend to our colleagues who made plasmids available through Addgene (\#46294 and \#47322). The project profited also from discussions at the International Atomic Energy Agency funded meetings of the Coordinated Research Project "Multifactorial reproductive sterility system to avoid resistance development against transgenic Sterile Insect Technique approaches". We acknowledge support by the Open Access Publication Funds of the Göttingen University. 


\section{References}

1. Hauser M. A historic account of the invasion of Drosophila suzukii (Matsumura) (Diptera: Drosophilidae) in the continental United States, with remarks on their identification. Pest Manag Sci. 2011;67(11):1352-7.

2. Cini A, Ioriatti C, Anfora G. A review of the invasion of Drosophila suzukii in Europe and a draft research agenda for integrated pest management. Bull Insectology. 2012;65(1):12.

3. Walsh DB, Bolda MP, Goodhue RE, Dreves AJ, Lee J, Bruck DJ, et al. Drosophila suzukii (Diptera: Drosophilidae): Invasive Pest of Ripening Soft Fruit Expanding its Geographic Range and Damage Potential. J Integr Pest Manag. 2011 Apr 1;2(1): G1-7.

4. Asplen MK, Anfora G, Biondi A, Choi D-S, Chu D, Daane KM, et al. Invasion biology of spotted wing Drosophila (Drosophila suzukii): a global perspective and future priorities. J Pest Sci. 2015 Sep;88(3):469-94.

5. Deprá M, Poppe JL, Schmitz HJ, De Toni DC, Valente VLS. The first records of the invasive pest Drosophila suzukii in the South American continent. J Pest Sci. 2014 Sep;87(3):379-83.

6. Lavagnino NJ, Díaz BM, Cichón LI, De la Vega GJ, Garrido SA, Lago JD, et al. New records of the invasive pest Drosophila suzukii (Matsumura) (Diptera: Drosophilidae) in the South American continent. Rev Soc Entomológica Argent. 2018 Mar 30;77(1):27-31.

7. Kenis M, Tonina L, Eschen R, van der Sluis B, Sancassani M, Mori N, et al. Non-crop plants used as hosts by Drosophila suzukii in Europe. J Pest Sci. 2016 Jul;89(3):735-48.

8. Lee JC, Bruck DJ, Curry H, Edwards D, Haviland DR, Van Steenwyk RA, et al. The susceptibility of small fruits and cherries to the spotted-wing drosophila, Drosophila suzukii. Pest Manag Sci. 2011 Nov;67(11):1358-67.

9. Lee JC, Bruck DJ, Dreves AJ, Ioriatti C, Vogt H, Baufeld P. In Focus: Spotted wing drosophila, Drosophila suzukii, across perspectives. Pest Manag Sci. 2011 Nov;67(11):1349-51.

10. Mazzi D, Bravin E, Meraner M, Finger R, Kuske S. Economic Impact of the Introduction and Establishment of Drosophila suzukii on Sweet Cherry Production in Switzerland. Insects. 2017 Feb 8;8(1):18.

11. Haviland DR, Beers EH. Chemical Control Programs for $<\mathrm{I}>$ Drosophila suzukii $</ \mathrm{I}>$ that Comply With International Limitations on Pesticide Residues for Exported Sweet Cherries. J Integr Pest Manag. 2012 Jun 1;3(2):1-6.

12. Diepenbrock LM, Rosensteel DO, Hardin JA, Sial AA, Burrack HJ. Season-long programs for control of Drosophila suzukii in southeastern U.S. blueberries. Crop Prot. 2016 Mar;81:76-84. 
13. Lee JC, Wang X, Daane KM, Hoelmer KA, Isaacs R, Sial AA, et al. Biological Control of Spotted-Wing Drosophila (Diptera: Drosophilidae) - Current and Pending Tactics. J Integr Pest Manag. 2019 Jan 1;10(1):13.

14. Leach H, Van Timmeren S, Isaacs R. Exclusion Netting Delays and Reduces Drosophila suzukii (Diptera: Drosophilidae) Infestation in Raspberries. J Econ Entomol. 2016 Oct;109(5):2151-8.

15. Rendon D, Hamby KA, Arsenault-Benoit AL, Taylor CM, Evans RK, Roubos CR, et al. Mulching as a cultural control strategy for Drosophila suzukii in blueberry. Pest Manag Sci [Internet]. 2019 Jun 17 [cited 2019 Jun 30];0(ja). Available from: https://onlinelibrary.wiley.com/doi/abs/10.1002/ps.5512

16. Knipling EF. Possibilities of Insect Control or Eradication Through the Use of Sexually Sterile Males1. J Econ Entomol. 1955 Aug 1;48(4):459-62.

17. Krafsur ES. Sterile Insect Technique for Suppressing and Eradicating Insect Population: 55 Years and Counting. J Agric Entomol. 1998 Oct;15(4):17.

18. Krafsur ES, Lindquist DA. Did the Sterile Insect Technique or Weather Eradicate Screwworms (Diptera: Calliphoridae) from Libya? J Med Entomol. 1996 Nov 1;33(6):877-87.

19. Horn C, Wimmer EA. A transgene-based, embryo-specific lethality system for insect pest management. Nat Biotechnol. 2003 Jan;21(1):64-70.

20. Schetelig MF, Caceres C, Zacharopoulou A, Franz G, Wimmer EA. Conditional embryonic lethality to improve the sterile insect technique in Ceratitis capitata (Diptera: Tephritidae). BMC Biol. 2009 Dec;7(1):4.

21. Ogaugwu CE, Schetelig MF, Wimmer EA. Transgenic sexing system for Ceratitis capitata (Diptera: Tephritidae) based on female-specific embryonic lethality. Insect Biochem Mol Biol. 2013 Jan;43(1):1-8.

22. Yan Y, Scott MJ. A transgenic embryonic sexing system for the Australian sheep blow fly Lucilia cuprina. Sci Rep. 2015 Dec;5(1):16090.

23. Scolari F, Schetelig MF, Bertin S, Malacrida AR, Gasperi G, Wimmer EA. Fluorescent sperm marking to improve the fight against the pest insect Ceratitis capitata (Wiedemann; Diptera: Tephritidae). New Biotechnol. 2008 Jun;25(1):76-84.

24. Smith RC, Walter MF, Hice RH, O’Brochta DA, Atkinson PW. Testis-specific expression of the ?2 tubulin promoter of Aedes aegypti and its application as a genetic sex-separation marker. Insect Mol Biol. 2007 Feb;16(1):61-71.

25. Zimowska GJ, Nirmala X, Handler AM. The $\beta 2$-tubulin gene from three tephritid fruit fly species and use of its promoter for sperm marking. Insect Biochem Mol Biol. 2009 Aug;39(8):508-15. 
26. Catteruccia F, Benton JP, Crisanti A. An Anopheles transgenic sexing strain for vector control. Nat Biotechnol. 2005 Nov;23(11):1414-7.

27. Ishino Y, Shinagawa H, Makino K, Amemura M, Nakata A. Nucleotide sequence of the iap gene, responsible for alkaline phosphatase isozyme conversion in Escherichia coli, and identification of the gene product. J Bacteriol. 1987 Dec;169(12):5429-33.

28. Makarova KS, Grishin NV, Shabalina SA, Wolf YI, Koonin EV. [No title found]. Biol Direct. 2006;1(1):7.

29. Barrangou R, Fremaux C, Deveau H, Richards M, Boyaval P, Moineau S, et al. CRISPR Provides Acquired Resistance Against Viruses in Prokaryotes. Science. 2007 Mar 23;315(5819):1709-12.

30. Garneau JE, Dupuis M-È, Villion M, Romero DA, Barrangou R, Boyaval P, et al. The CRISPR/Cas bacterial immune system cleaves bacteriophage and plasmid DNA. Nature. 2010 Nov;468(7320):67-71.

31. Jinek M, Chylinski K, Fonfara I, Hauer M, Doudna JA, Charpentier E. A Programmable DualRNA-Guided DNA Endonuclease in Adaptive Bacterial Immunity. Science. 2012 Aug 17;337(6096):816-21

32. Bassett AR, Tibbit C, Ponting CP, Liu J-L. Highly Efficient Targeted Mutagenesis of Drosophila with the CRISPR/Cas9 System. Cell Rep. 2013 Jul 11;4(1):220-8.

33. Hwang WY, Fu Y, Reyon D, Maeder ML, Tsai SQ, Sander JD, et al. Efficient genome editing in zebrafish using a CRISPR-Cas system. Nat Biotechnol. 2013 Mar;31(3):227-9.

34. Platt RJ, Chen S, Zhou Y, Yim MJ, Swiech L, Kempton HR, et al. CRISPR-Cas9 Knockin Mice for Genome Editing and Cancer Modeling. Cell. 2014 Oct;159(2):440-55.

35. Hall B, Cho A, Limaye A, Cho K, Khillan J, Kulkarni AB. Genome Editing in Mice Using CRISPR/Cas9 Technology. Curr Protoc Cell Biol. 2018;81(1):e57.

36. Gratz SJ, Cummings AM, Nguyen JN, Hamm DC, Donohue LK, Harrison MM, et al. Genome Engineering of Drosophila with the CRISPR RNA-Guided Cas9 Nuclease. Genetics. 2013 Aug;194(4):1029-35.

37. Li M, Bui M, Yang T, Bowman CS, White BJ, Akbari OS. Germline Cas9 expression yields highly efficient genome engineering in a major worldwide disease vector, Aedes aegypti. Proc Natl Acad Sci. 2017 Dec 5;114(49):E10540-9.

38. Li M, Akbari OS, White BJ. Highly Efficient Site-Specific Mutagenesis in Malaria Mosquitoes Using CRISPR. G3. 2018 Feb;8:653-8. 
39. Jiang J, Zhang L, Zhou X, Chen X, Huang G, Li F, et al. Induction of site-specific chromosomal translocations in embryonic stem cells by CRISPR/Cas9. Sci Rep. 2016 Apr;6(1):21918.

40. Iwata S, Yoshina S, Suehiro Y, Hori S, Mitani S. Engineering new balancer chromosomes in C. elegans via CRISPR/Cas9. Sci Rep. 2016 Dec;6(1):33840.

41. Port F, Bullock SL. Augmenting CRISPR applications in Drosophila with tRNA-flanked sgRNAs. Nat Methods. 2016 Oct;13(10):852-4.

42. Port F, Chen H-M, Lee T, Bullock SL. Optimized CRISPR/Cas tools for efficient germline and somatic genome engineering in Drosophila. Proc Natl Acad Sci. 2014 Jul 22;111(29):E2967-76.

43. Li F, Scott MJ. CRISPR/Cas9-mediated mutagenesis of the white and Sex lethal loci in the invasive pest, Drosophila suzukii. Biochem Biophys Res Commun. 2016 Jan;469(4):911-6.

44. Kalajdzic P, Schetelig MF. CRISPR/Cas-mediated gene editing using purified protein in Drosophila suzukii. Entomol Exp Appl. 2017 Sep;164(3):350-62.

45. Li J, Handler AM. Temperature-dependent sex-reversal by a transformer-2 gene-edited mutation in the spotted wing drosophila, Drosophila suzukii. Sci Rep. 2017 Dec;7(1):12363.

46. Karageorgi M, Bräcker LB, Lebreton S, Minervino C, Cavey M, Siju KP, et al. Evolution of Multiple Sensory Systems Drives Novel Egg-Laying Behavior in the Fruit Pest Drosophila suzukii. Curr Biol. 2017 Mar 20;27(6):847-53.

47. Eckermann KN, Dippel S, KaramiNejadRanjbar M, Ahmed HM, Curril IM, Wimmer EA. Perspective on the combined use of an independent transgenic sexing and a multifactorial reproductive sterility system to avoid resistance development against transgenic Sterile Insect Technique approaches. BMC Genet. 2014;15(Suppl 2):S17.

48. Kemphues J, Kaufman C, Raff A, Raff C. The Testis-Specific P-Tubulin Subunit in Drosophila melanogaster Has Multiple Functions in Spermatogenesis. Cell. 1982 Dec;31:655-70.

49. Michiels F, Gasch A, Kaltschmidt B, Renkawitz-Pohl R. A 14 bp promoter element directs the testis specificity of the Drosophila 32 tubulin gene. EMBO J. 1989;8(5):1559-65.

50. Michiels F, Wolk A, Renkawitz-Pohl R. Further sequence requirements for male germ cellspecific expression under the control of the 14 bp promoter element (B2UE1) of the Drosophila B2 tubulin gene. Nucleic Acids Res. 1991 Jul 26;19(16):4515-21.

51. Galizi R, Doyle LA, Menichelli M, Bernardini F, Deredec A, Burt A, et al. A synthetic sex ratio distortion system for the control of the human malaria mosquito. Nat Commun. 2014 Sep;5(1):3977. 
52. Schetelig MF, Handler AM. A transgenic embryonic sexing system for Anastrepha suspensa (Diptera: Tephritidae). Insect Biochem Mol Biol. 2012 Oct;42(10):790-5.

53. Kondo S, Ueda R. Highly Improved Gene Targeting by Germline-Specific Cas9 Expression in Drosophila. Genetics. 2013 Nov 1;195(3):715-21.

54. Shoji W, Sato-Maeda M. Application of heat shock promoter in transgenic zebrafish. Dev Growth Differ. 2008 Aug;50(6):401-6.

55. Schulte C, Leboulle G, Otte M, Grünewald B, Gehne N, Beye M. Honey bee promoter sequences for targeted gene expression: Honey bee promoter analysis. Insect Mol Biol. 2013 Aug;22(4):399-410.

56. Lécuyer E. High Resolution Fluorescent In Situ Hybridization in Drosophila. In: Gerst JE, editor. RNA Detection and Visualization [Internet]. Totowa, NJ: Humana Press; 2011 [cited $2019 \mathrm{Jul}$ 4]. p. 31-47. Available from: http://link.springer.com/10.1007/978-1-61779-005-8_3

57. Zimmerman SG, Peters NC, Altaras AE, Berg CA. Optimized RNA ISH, RNA FISH and protein-RNA double labeling (IF/FISH) in Drosophila ovaries. Nat Protoc. 2013 Nov;8(11):2158-79.

58. Eckermann KN, Ahmed HMM, KaramiNejadRanjbar M, Dippel S, Ogaugwu CE, Kitzmann P, et al. Hyperactive piggyBac transposase improves transformation efficiency in diverse insect species. Insect Biochem Mol Biol. 2018 Jul 1;98:16-24.

59. Horn C, Wimmer EA. A versatile vector set for animal transgenesis. Dev Genes Evol. 2000 Nov $1 ; 210(12): 630-7$.

60. Ulrich A, Andersen KR, Schwartz TU. Exponential Megapriming PCR (EMP) CloningSeamless DNA Insertion into Any Target Plasmid without Sequence Constraints. Isalan M, editor. PLoS ONE. 2012 Dec 31;7(12):e53360.

61. Paix A, Folkmann A, Rasoloson D, Seydoux G. High Efficiency, Homology-Directed Genome Editing in Caenorhabditis elegans Using CRISPR-Cas9 Ribonucleoprotein Complexes. Genetics. 2015 Sep;201(1):47-54.

62. Vouillot L, Thélie A, Pollet N. Comparison of T7E1 and Surveyor Mismatch Cleavage Assays to Detect Mutations Triggered by Engineered Nucleases. G3amp58 GenesGenomesGenetics. 2015 Mar;5(3):407-15.

63. Huang MC, Cheong WC, Lim LS, Li M-H. A simple, high sensitivity mutation screening using Ampligase mediated T7 endonuclease I and Surveyor nuclease with microfluidic capillary electrophoresis. Electrophoresis. 2012 Mar;33(5):788-96. 
64. Engler C, Kandzia R, Marillonnet S. A One Pot, One Step, Precision Cloning Method with High Throughput Capability. El-Shemy HA, editor. PLoS ONE. 2008 Nov 5;3(11):e3647.

65. Engler C, Gruetzner R, Kandzia R, Marillonnet S. Golden Gate Shuffling: A One-Pot DNA Shuffling Method Based on Type IIs Restriction Enzymes. Peccoud J, editor. PLoS ONE. 2009 May 14;4(5):e5553. 


\section{Figures}

A

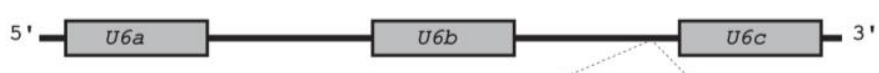

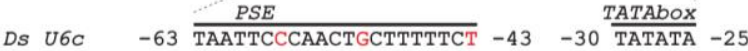

Dm U6:3 -63 TAATTCTCAACTTCTTTTTCC $-43 \quad-30$ TATATA -25

B

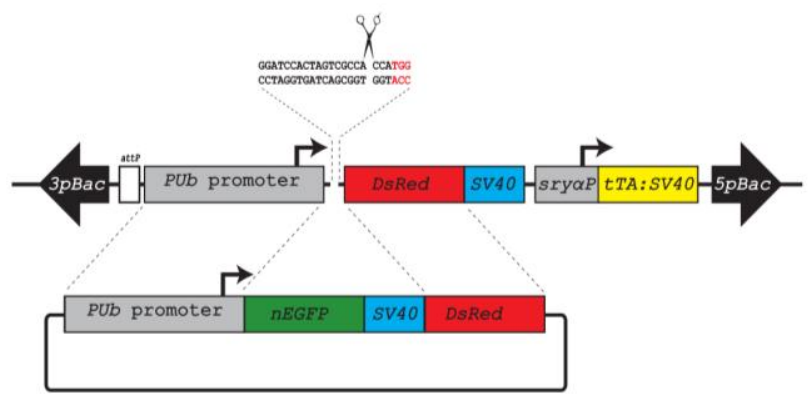

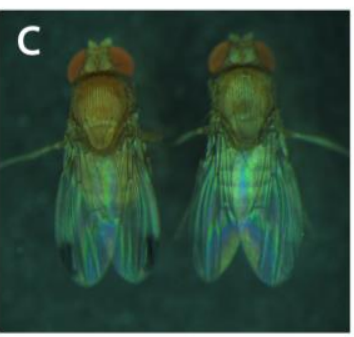

D

E
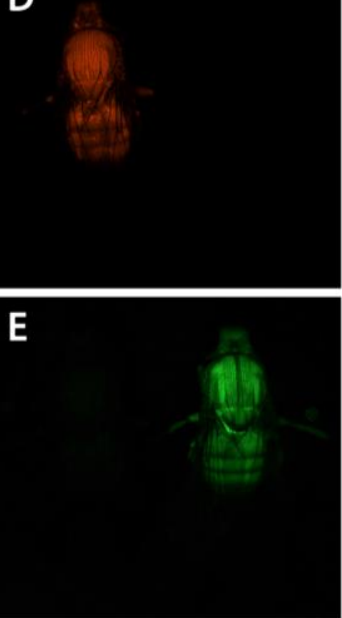

$\mathbf{F}$

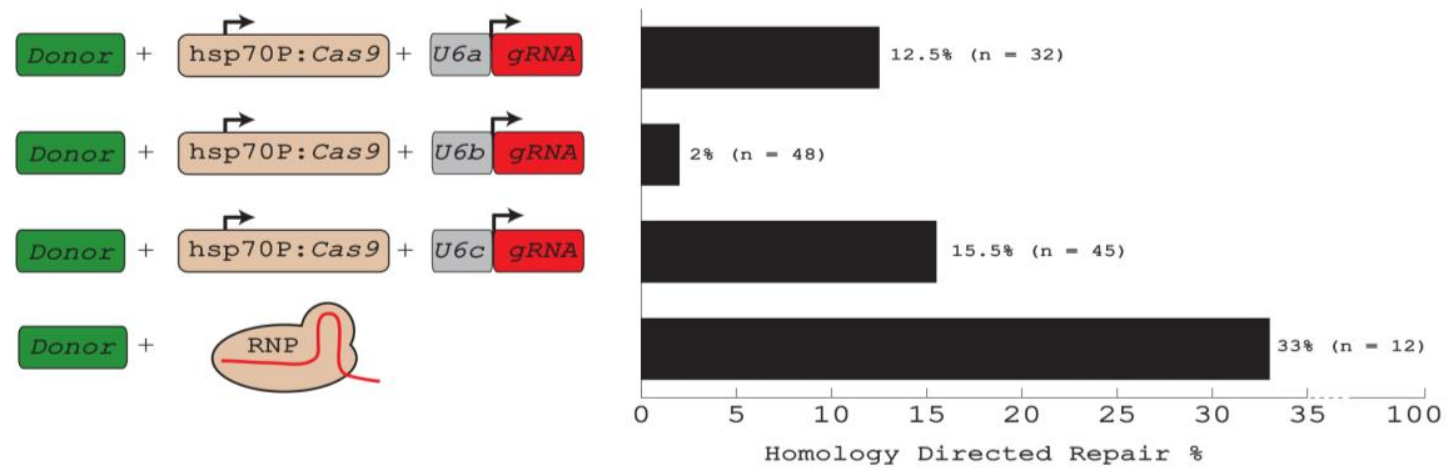

Fig. 1 Improvement of genome editing in D. suzukii. (A) Three copies of the $s n R N A$ gene $U 6$ in the genome of $D$. suzkuii. The transcription from U6 genes by RNA pol III is directed by the proximal sequence element PSE which is highly conserved between D. suzukii and D. melanogaster. (B) Scheme for HDR-based genome editing at a transgenic target platform. Sequence of the target site in the transgenic strain showing the PAM sequence in red. The scissors indicate where Cas9 induces the DSB three nucleotides upstream of the PAM. (C-E) Fluorescent marker change as the result of the HDR knock-in: images of two male flies taken with cold light (C), RFP fliter $(\mathbf{D})$, and EYFP filter $(\mathbf{E})$. (F) Comparison of Ds U6a, $U 6 b, U 6 c$ promoters as well as RNP in their efficiency to promote HDR-mediated knock-ins. 
(A)

$D s \beta 2 t$

\begin{tabular}{|l|l|l|l|}
+1 & & \\
5 ' UTR & ExonI & ExonII \\
\hline
\end{tabular}

\begin{tabular}{|l|l|l|}
+1 & & \\
5
\end{tabular}

(B)

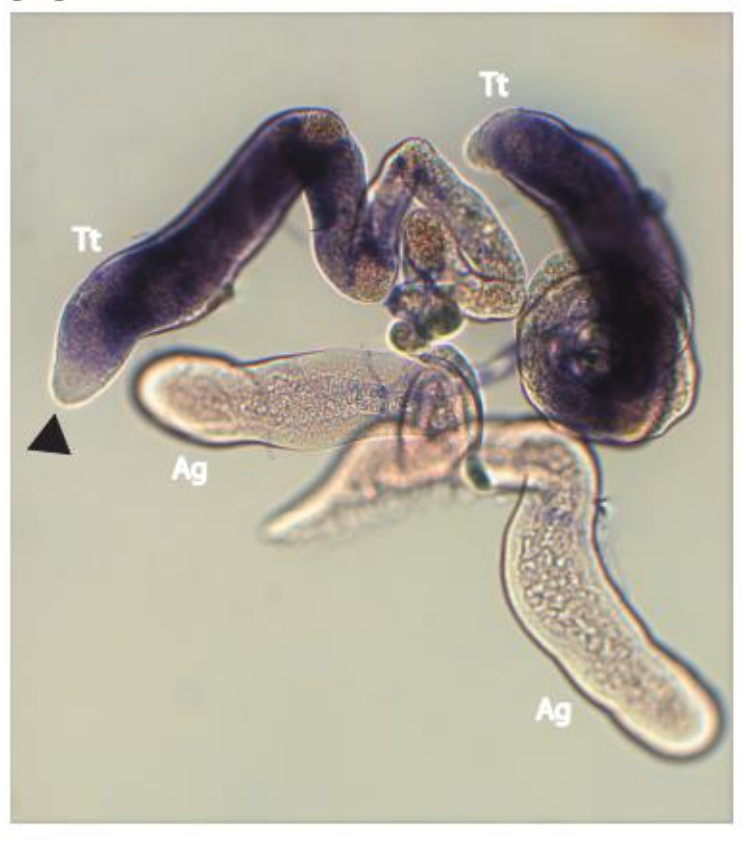

(C)

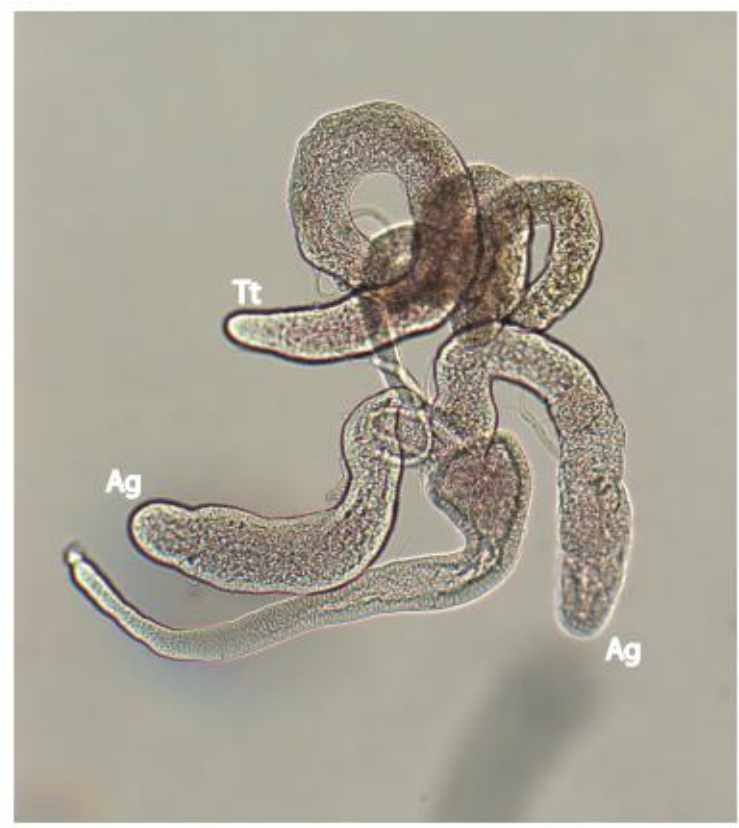

Fig. 2 D. suzukii $\beta 2 t$ gene and its expression. (A) $D s \beta 2 t$ gene has two exons and one intron similar to D. melanogaster. The gene is slightly longer in $D$. suzukii due to increase in the size of the 5'UTR and the intron. The numbers indicate the first nucleotide of the respective feature relative to the first transcribed nucleotide. (B) Testes whole mount in situ hybridization using DIG labeled RNA antisense probe against Ds $\beta 2 t 5$ 'UTR and exon I detects strong and testes-specific expression. The gene is not expressed at the tip of the testes (black triangle) where stem cells reside. (C) Negative control using DIG labeled sense probe shows no signs of staining. The abbreviations $\mathrm{Tt}$ and Ag refer to testes or the accessory glands, respectively. 
A D.suzuki1 ATCOAGACCTA--5n--GAACATTC--20n--TTCAGTT--46n--AAAATTATACGTPTAAAT +68

B
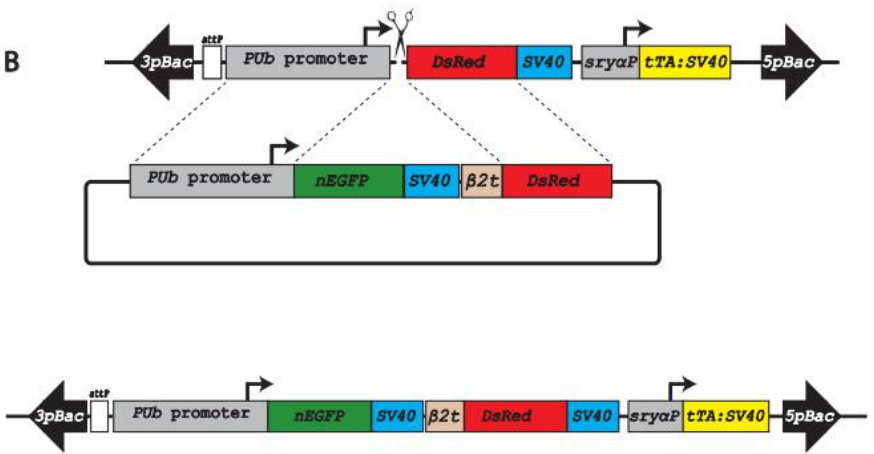
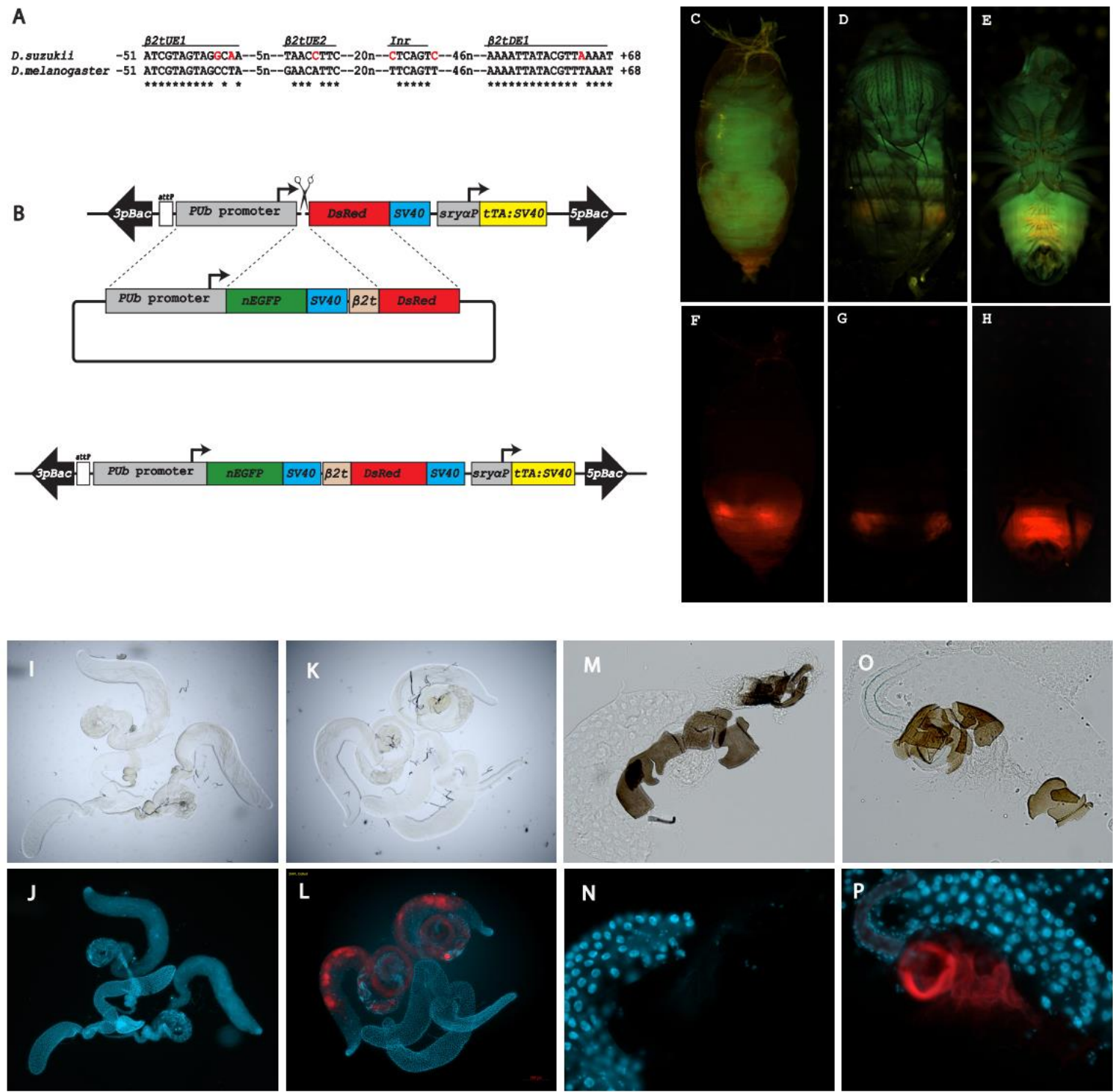

Fig. 3 Generation of a sperm marking strain. (A) Drosophila $\beta 2 t$ genes have a very short and highly conserved promoter/enhancer region with a $14 \mathrm{bp}$ upstream element $(\beta 2 t U E 1)$ that confers testes-specific expression while the other indicated elements play quantitative roles. (B) Scheme for HDR knock-in of the repair template having EGFP:SV4O and $\beta 2 t$ promoter fused to DsRed. (C-H) Result of the HDR knock-in: images of Pupae (C, F) as well as adult males in dorsal (D, G) or ventral view $(\mathbf{E}, \mathbf{H})$ taken with GFP-LP $(\mathbf{C}-\mathbf{E})$ or RFP $(\mathbf{F}-\mathbf{H})$ filters, respectively. Compared to wild type $(\mathbf{I}, \mathbf{J})$, the testes of the knock-in males show strong expression of DsRed under control of the $\beta 2 t$ promoter $(\mathbf{K}, \mathbf{L})$. In contrast to wild type females mated to wild type males $(\mathbf{M}, \mathbf{N})$, the fluorescent sperm can also be detected in the storage organ (spermatheca) of wild type females mated to the transgenic sperm-marked strain $(\mathbf{O}, \mathbf{P})$. I, K, M, O images were taken under bright field, and J, L, N, P are composites of images made of the same objects using a DAPI and a DsRed filter. 
Additional File 1: Supplementary Table 1: List of primers used.

\begin{tabular}{|c|c|c|}
\hline Code & Name & Sequence $5^{\prime}-3{ }^{\prime}$ \\
\hline HM\#23 & AgeI_srya_F4 & GAACCGGTGTGCACAAGCGAGTCCACCAG \\
\hline $\mathrm{HM} \# 24$ & NheI_Srya_R & CCGCTAGCCCTAGGAGCTCTATAAGATGTGCT \\
\hline HM\#25 & Ds_B2t_F1 & AAGATGCGTGAAATCGTGCACATTCAG \\
\hline $\mathrm{HM} \# 26$ & Ds_B2t_R1 & TTATTCATCGCCGCCACCCTCTTC \\
\hline $\mathrm{HM} \# 33$ & Ds_B2t_GSP & GTCCGGCCTGAATGTGCACGATTTCACGC \\
\hline HM\#37 & $\mathrm{Hma}-44 \mathrm{~F}$ & ACTCATCGATATGGCCTCCTCCGAGGACGTCATC \\
\hline $\mathrm{HM} \# 41$ & B2t5UTR-F1 & AGTCCACCCTAGTATCAGCTAGCAAGCACACG \\
\hline $\mathrm{HM} \# 55$ & HindIII-T7-BbsI-F & AGCTTGATGTGAATTGTAATACGACTCACTATAGGGTCTTCGAGAAGACCT \\
\hline HM\#56 & BbsI-T7-HindIII-R & AAACAGGTCTTCTCGAAGACCCTATAGTGAGTCGTATTACAATTCACATCA \\
\hline HM\#69 & DsRed-mega-R & GGAAGGACAGCTTCTTGTAGTCGGGG \\
\hline $\mathrm{HM} \# 73$ & hspEcoRIF2 & GCGAATTCTCCAGAAACTCAAACAGAAACC \\
\hline $\mathrm{HM} \# 75$ & Dshsp-ClaIR & GCATATCGATTTGTGTGTTTGTGTTTGTGGATGCAGTTG \\
\hline $\mathrm{HM} \# 84$ & chiRNA_R & AAAAAGCACCGACTCGGTGCCACTTTTTCAAGTTG \\
\hline HМ\#104 & DsU6MegaF & $\begin{array}{l}\text { CGAGGTCGACGGTATCGATAAGCTTGTTCCTTGCTAACAATATCTTTTTATGTC } \\
\text { ATTTCC }\end{array}$ \\
\hline HM\#127 & HM_pSL_F & CGAAAGGGGGATGTGCTGCAAGGCGATTAAG \\
\hline HM\#128 & HM_pSL_R & CCGGCTCGTATGTTGTGTGGAATTGTG \\
\hline HM\#137 & U6_neuF2 & CGTTTGGGAGCAGGGCTTCACCTTCCTGG \\
\hline HM\#138 & U6_neuR2 & GCACTCGTATCACCCACAGCGATGAACACG \\
\hline HM\#152 & pET_BsaI_BsaIF & TATGGAGACCGTCGACCTAGGGAGACC \\
\hline HM\#153 & pET_BsaI_BsaIR & TCGAGGTCTCCCTAGGTCGACGGTCTCCA \\
\hline HM\#158 & DsU6MegaR2 & $\begin{array}{l}\text { CTCTAAAACAGGTCTTCTCGAAGACCCGAAGTTCAAGTGAGATTCTTCCCTATT } \\
\text { TATATTG }\end{array}$ \\
\hline HМ\#159 & U6a_Mega_R2 & $\begin{array}{l}\text { CTCTAAAACAGGTCTTCTCGAAGACCCGAAGTTCAAGTGGGTTTCTTCCCTATT } \\
\text { TATACTGC }\end{array}$ \\
\hline HМ\#160 & U6c_Mega_R2 & $\begin{array}{l}\text { CTCTAAAACAGGTCTTCTCGAAGACCCGATTTCGTATTGAAAAATGTCGTATAT } \\
\text { ATACTACC }\end{array}$ \\
\hline HM\#161 & Red_T1F & CTTCGGATCCACTAGTCGCCACCA \\
\hline HM\#162 & Red_T1R & AAACTGGTGGCGACTAGTGGATCC \\
\hline HМ\#169 & Red_T1_CF & AATCGGATCCACTAGTCGCCACCA \\
\hline HM\#167 & Red_T5F & CTTCGAACAGGTGGTGGCGGCCCT \\
\hline HM\#180 & NdeI_Histag_F & TATGCATCATCATCATCACCA \\
\hline HM\#181 & NdeI_Histag_R & TATGGTGATGATGATGATGCA \\
\hline HМ\#186 & U6_Down_MegaF & $\begin{array}{l}\text { CTTGAAAAAGTGGCACCGAGTCGGTGCTTTTTTTGCAAACCTGAGGGTGAATAT } \\
\text { TCAAAC }\end{array}$ \\
\hline HM\#187 & U6_Down_MegaR & $\begin{array}{l}\text { GTGGATCCCCCGGGCTGCAGGAATTCGATATGTATGCGTTAAGCAAAAATTGAA } \\
\text { AACATAAC }\end{array}$ \\
\hline HМ\#192 & PUbT7 & CGAACGCACTCGAGCATTTGTGTGCATG \\
\hline HM\#215 & T7_Red1F & TATAGGATCCACTAGTCGCCACCA \\
\hline HM\#221 & Red1_mutG2AF & CATGATGAGCAAGGGCGAGGAGCTGTTC \\
\hline $\mathrm{HM} \# 222$ & Red1_mutG2AR & GCTCATCATGGTGGCGACCGGTGGATC \\
\hline $\mathrm{HM} \# 252$ & B2t-DsRedR & GATGACGTCCTCGGAGGAGGCCATCTTAACCGACTGTCAAGGAAC \\
\hline HM\#285 & SV40-B2tF & CATCAATGTATCTTAAACTCATCGATATCGTAGTAGGCAAGCTAATAACC \\
\hline HM\#358 & U6a_Mega_F & CGAGGTCGACGGTATCGATAAGCTTTCCGCCGAGTCGCCCATCAGCGAGGAGG \\
\hline HM\#360 & U6c_Mega_F & $\begin{array}{l}\text { CGAGGTCGACGGTATCGATAAGCTTGTTCCTTAAACACTCAATATTTTTATAAT } \\
\text { CTGC }\end{array}$ \\
\hline
\end{tabular}




\section{Additional File 2: piggyBacinsertion in D. suzukii line 06_F5M2}

piggyBac insertion in the second intron of a gene referred to as Suppressor of Under-Replication (SuUR). Underlined are the restriction sites for EcoRI and Xhol, respectively. The bold TTAA sequence represents the site of piggyBac integration, which was duplicated.

TTCGCGAATTCTGCATCCTGAACGATGAGAGTGGCCTGGGTAAACTGGCCACGGTGGCGGCACTTC TCAGTGCCCTAGATCCCGCCAAGAAAACTCTCATTGTGCTGCAGAACGACGAGCAACTGCTCGCTG GTTGGCGGTTCCATCTGGACACACTCACGAACCTGCAGGTGTACACCATTCAAGGAGTCCAAGGTA ATGCTCTGCGTTTAATGATATTCTTGGATTTCTATGTGGATTAGAATTTGGAAAACACCATGTTCT GATATTTTTATACTTCTCCTAAATCAACATTCCTTGATAGTTAGTTTCGAAGAATGAATCTCWCTG CAGCGTAAAATGMYTATATTTTGAAAACCACAAAATTGATCAGTTTTTTATTTTGTGTGTACAATT TCGCAATTAAACGAAAGTAGTGGGTTCCATTTAAGGGATCTGTTAAAATTTGATTTTACCAACTTT ATTTTGCTATACGGTCTGAACCATTTTAAATAATTTTTTATTATAGTTTAGTTTATAGTTTTATTT ACAAAATAAATAATGAATATTCTTTAAACTGCTAAAACAATTTGAAAAATTAACATTATTTCCATA TAATCAATTTTTAAAACGAAAACTTGTTTAA_5'piggYBac3'_TTAATTAAGTCTTGTATAAAC CTTATCAAAGAACTATATATTTTTATTCGGAAACTAACAGTTTGTTTTACCTCCTTTACAGACACC ACAGACTCCCCGCACAACGTTTACCTGGCAAAGTGGAGCCAGTTACGCAGCATTGGAGATCTCAGT CGCCTCAAGTTCGACTACGTTCTGGTAGACAATCGGGGCCACACGCTGAACAACAGCTTCTGCACA TCAATGCTTCTCAAGCATTTTGAGGGAAGGGTAAACATTCTTATCTCCAGTGTTGACATTACGGTG AGGCAATGCACCCATAAGTGGTTCACATGGAGCTTTATTTAGTTTGTTTCCCTATCTGCAGTCAGA CGTAAGGTTGCTGTTCATGTTTTGCGGTTGGGCGGGCGCCTGGAGCATCAGTATCGGAGCTTCCAG AGCTTCGACCGCAAGTTCCATTTGCCAGATCCAAAGGAGGTCTTTAGCAAGCGTATAGATCTCGAG AT 


\subsection{Improvement on the genetic engineering of an invasive agricultural pest insect, the cherry vinegar fly, Drosophila suzukii}

To be able to develop any transgene-based improvement of the sterile insect technique (SIT) as a pest control strategy, it is necessary to have at least one relaible and efficient tool for germline transformation. In this part, we present an improvement on piggyBac germline tranformation with regard to the suitability of different $D$. suzukii strains and identified the AM strain as the most suitable. In addition, we present for the first time the use of site-specific germline transformation based on the phage $\varphi \mathrm{C} 31$ integrase system that will facilitate testing different trangenes in the same genomic environment and can be used to stabilize transgenes generated by transposon-based vectors. We also showed for the first time that $\varphi \mathrm{C} 31 \mathrm{RMCE}$ works in D. suzukii. Moreover, the tet-off binary system was tested.

This chapter thus provides a set of genome manipulation tools for the fruit pest $D$. suzukiithat should enable development of transgene-based pest control strategies necessary for the improvement of the SIT.

Hassan M. M. Ahmed, Fabienne Heese, \& Ernst A. Wimmer

Authors contributions:

Hassan M. M. Ahmed: Isolation of genes, in situ hybridization, generation of constructs, generation of the transgenic attP self-docking lines, generation of $t T A$ driver lines, testing of the tet-Off binary system, RMCE analysis. Writing of first draft of manuscript and composition of all figures.

Fabienne Heese:

Generation of transgenic attP docking lines for RMCE and Cas9 responder line.

Status of manuscript: Accepted for publication in BMC Genetics 
Improvement on the genetic engineering of an invasive agricultural pest insect, the cherry vinegar fly, Drosophila suzukii

\author{
Hassan M. M. Ahmed ${ }^{1,2}$, Fabienne Heese $^{1}$, \& Ernst A. Wimmer ${ }^{1 *}$ \\ ${ }^{1}$ Department of Developmental Biology, Johann-Friedrich-Blumenbach-Institute of Zoology and Anthropology, \\ Göttingen Center for Molecular Biosciences, Georg-August-University Göttingen, 37077 Göttingen, Germany. \\ ${ }^{2}$ Department of Crop Protection, Faculty of Agriculture-University of Khartoum P.O. Box 32, Postal Code 13314 \\ Khartoum North, Khartoum, Sudan \\ *Corresponding Author: E. A. Wimmer. Tel. +49 5513928666 \\ E-Mail address: ewimmer@gwdg.de (E.A. Wimmer).
}

\begin{abstract}
Background: The invasive fly Drosophila suzukii has become an established fruit pest in Europe, the USA, and South America with no effective and safe pest management. Genetic engineering enables the development of transgene-based novel genetic control strategies against insect pests and disease vectors. This, however, requires the establishment of reliable germline transformation techniques. Previous studies have shown that $D$. suzukii is amenable to transgenesis using the transposon-based vectors piggyBac and Minos, site-specific recombination (lox/Cre), and CRISPR/Cas9 genome editing.
\end{abstract}

Results: We experienced differences in the usability of piggyBac-based germline transformation in different strains of $D$. suzukii: we obtained no transgenic lines in a US strain, a single rare transgenic line in an Italian strain, but observed a reliable transformation rate of 2.5 to $11 \%$ in a strain from the French Alps. This difference in efficiency was confirmed by comparative examination of these three strains. In addition, we used an attPlanding site line to successfully established $\varphi \mathrm{C} 31$-integrase-mediated plasmid integration at a rate of $10 \%$ and generated landing site lines with two attP sequences to effectively perform $\varphi \mathrm{C} 31$ Recombinase Mediated Cassette Exchange ( $\varphi$ C31-RMCE) with 11\% efficiency. Moreover, we isolated and used the endogenous regulatory regions of $D s$ nanos to express $\varphi \mathrm{C} 31$ integrase maternally to generate self-docking lines for $\varphi \mathrm{C} 31-\mathrm{RMCE}$. Besides, we isolated the promoter/enhancer of $D$ s serendipity $\square$ to drive the heterologous tetracycline-controlled transactivator ( $t T A$ ) during early embryonic development and generated a testes-specific tTA driver line using the endogenous beta-2-tubulin $(\beta 2 t)$ promoter/enhancer.

Conclusion: Our results provide evidence that the $D$. suzukii strain AM derived from the French Alps is more suitable for piggyBac germline transformation than other strains. We demonstrated the feasibility of using $\varphi \mathrm{C} 31-\mathrm{RMCE}$ in the cherry vinegar fly and generated a set of lines that can be used for highly efficient integration of larger constructs. The $\varphi \mathrm{C} 31-$ based integration will facilitate modification and stabilization of previously generated 
transgenic lines that carry at least one attPsite in the transgene construction. An early embryospecific and a spermatogenesis-specific driver line were generated for future use of the binary expression system tet-off to engineer tissue- and stage-specific effector gene expression for genetic pest control strategies.

Keywords: Binary expression system, enhancer/promoter, insect transgenesis, molecular entomology, pest management, Spotted Wing Drosophila, Sterile Insect Technique.

\section{Background}

The invasive pest Drosophila suzukii commonly referred to as the cherry vinegar fly or the spotted wing Drosophila (SWD) originated from East Asia [1, 2]. It was described for the first time in Japan in 1916. In 2008, the fly has concomitantly been reported in Europe (Spain and Italy) and the USA (California), where the SWD presents a major threat to the soft and stone fruit industry [1-3]. The fly is armed with a prominent serrated ovipositor that enables it to lay eggs inside ripening intact fruits. The larvae eat and develop inside the fruits and lead to a crop loss of up to $100 \%$ [4]. Several insecticides have been used to control the fly with limited degrees of success [5, 6]. A genetic control method, the Sterile Insect Technique (SIT), might provide the most promising pest management strategy. SIT was proposed more than 75 years ago as biological control method to fight agricultural pests and diseases vectors. It is a speciesspecific birth control strategy, which makes it safe for pollinators and natural enemies and is thus environmentally friendly [7]. The SIT consists of mass rearing of the target pest in large numbers, sexing, sterilization of the males and successive inundative release in the target area. Genetic engineering offers different approaches for improvement of SIT [8-13]. For example, a transgene-based conditional embryonic lethality system was developed as a way to induce reproductive sterility, which can replace the need for ionizing radiation and ensure production of competent males [9, 10]. A transgenic female-specific embryonic lethality system developed for several dipterans, notably tephritid fruit flies, serves a method to eliminate females during early embryonic development and facilitates the production of only males for SIT releases [11-14].

The ability to genetically manipulate biological systems from mammalian and insect cell lines to insects and mouse has been revolutionized by the discovery and utilization of the most versatile transposon, piggyBac [15-17]. It belongs to the class II DNA transposons, which work by a cut and paste mechanisms [18]. piggyBac-based vectors were generated to insert cargo sequences at a TTAA recognition sequence in the genome of the target species. piggyBac-based germline transformation has been successfully established for many model and no-model organisms including Drosophila melanogaster [19-21], Ceratitis captitata [22, 23], Anastrepha suspensa [24], Drosophila suzukii [25], Anopheles gambiae [26], Aedes aegypti [27], Musca domestica [28], among others. The increase in the efficiency of germline transformation due to the use of a hyperactive version of the piggyBac transposase was 
demonstrated in several insects [23]. An inherent characteristic of transposon vectors using piggy $B a c$ is the random integration in the genome which makes them a useful tool for mutagenesis screens, enhancer traps, and exon traps [19, 29-31]. Also, in cases, when no clear target sequence can be identified, the random integration might result in a set of insertions, from which to choose the most suitable ones. However, this randomness is considered a drawback, when different transgenes were to be compared in the same genomic context [32, 33].

Site-specific recombinases (SSR) offer a more precise approach for genetic engineering of biological systems $[34,35]$. In the presence of the respective recombinase, recombination takes place between two identical sequences in case of Flp/FRT and Cre/lox [36, 37] or nonidentical sequences in case of $\varphi \mathrm{C} 31 \mathrm{att} P / \mathrm{attB}$ [38]. The use of SSR necessitates the generation of landing site lines by integrating at least a single landing site ( $F R T$, lox or attP) into the genome of the target species. This is routinely done by including the sequence within a transposon vector and integrate it randomly in the genome. Once generated, these landing sites can be used repeatedly to integrate different transgenes. In case a single landing site is integrated, the transgene of interest has to be delivered in a plasmid vector that has the respective recombinase recognition sequence which leads to integration of the whole plasmid including the antibiotic resistance gene. To avoid this, two landing sites can be placed close to each other into the genome ideally separated by a marker. The transgene to be inserted has to be flanked by two recombinase recognition sequences, which facilitate double recombination events leading to a recombinase mediated cassette exchange (RMCE). The $\varphi \mathrm{C} 31$-based integration and RMCE have been established in many insects for either modification and or stabilization of previously generated transgenes [39] or for site-specific germline transformation, which allows examination of different transgenes in the same genomic context [40]. Furthermore, the use of the $\varphi \mathrm{C} 31$ system allows for large transgenes to be integrated. In fact, BAC constructs of up to $133 \mathrm{~kb}$ were integrated using this system [41]. Moreover, in Drosophila and mosquitoes the $\varphi \mathrm{C} 31$ system has been used to generate self-docking strains that expresses the integrase from the enhancer/promoter of the maternal effect gene nanos. This has remarkably improved the efficiency of site-directed germline transformation $[42,43]$.

To generate transgene-based reproductive sterility or sexing strains, food supplementcontrolled binary expression systems have widely been used for conditional and tissue- or stage-specific gene expression [8-14]. The UAS/Gal4 system has intensively been used in $D$. melanogaster to drive tissue-specific expression of dsRNA to knockdown genes and study their function $[44,45]$. The tet system has initially been developed to be used in human cell culture and has since been engineered into tet-off and tet-on systems [46-48]. In insect biotechnology, the tet-off system was used e.g. to control the expression of effector molecules such as the proapoptotic gene head involution defective (hid), which leads to apoptotic cell 
death [8]. To drive the heterologous transactivator of such a binary expression system to cause effective reproductive sterility [9, 10] or female-specific killing [11, 13, 14, 49] based on early embryonic lethality, the promoter/enhancers of cellularization-specific genes need to be identified and isolated. Moreover, to direct sperm-specific expression for transgenic marking [50-52] or the development of multifactorial reproductive sterility [53], the use of promoters/enhancers active during spermatogenesis are of interest.

Here we show that $D$. suzukii strains originated from different locations can be transformed using piggyBac germline transformation with varying efficiency. In addition, we demonstrate the successful use of $\varphi \mathrm{C} 31$-based site-specific germline transformation both by integration in one attP site or by RMCE. Moreover, we provide a set of $D$. suzukii self-docking lines expressing $\varphi \mathrm{C} 31$ integrase maternally during oogenesis. Furthermore, we provide an early embryo-specific and a spermatogenesis-specific driver line for using the tet-off binary expression system to drive tissue-specific expression of effector genes.

\section{Results}

\section{Comparison of piggyBac germline transformation in different $D$. suzukii strains}

Transposon-based vectors have been intensively used for genetic manipulation from cell culture to mouse. The vector piggyBachas gained particular attention due to its versatility and usability in different systems. When we started to use piggyBac for germline transformation of an Italian strain of $D$. suzukii, we had only poor success and retrieved a rare transgenic line (06_F5M2) carrying construct HMMA006 [52], which mediates early embryonic expression of $t$ TA (Fig. 1), with a transformation rate of 1.6\% (300 embryos injected, 200 survived, 60 fertile, 1 transgenic line). However, several previous attempts with the same construct and additional attempts with five other constructs were unsuccessful. Changing to a US strain did not improve our approach, since trying the same five different constructs in this strain did not yield any transgenic lines. Only once we changed to the strain Alpes Maritimes (AM) isolated from the French Alps [54], we started to get reliable piggyBac germline transformation to work. In this strain, we regularly obtained transgenic lines for three different constructs with transformation rates between 2.5 and 11\% (Additional file 1): The testes-specific driver construct HMMA389, which is designed to be also useable for $\varphi \mathrm{C} 31-$ mediated RMCE and mediates spermatogenesis-specific expression of $t T A$ (Fig. 2); the DsRed-marked construct HMMA185 containing two attP sites for $\varphi \mathrm{C} 31$-mediated RMCE (Fig. 3); as well as the construct HMMA223 to generate self-docking lines for $\varphi \mathrm{C} 31$-mediated RMCE (Fig. 4). Additional file 2 provides a list of the obtained lines.

To examine the suitability of the three different strains for piggyBac germline transformation in a truly comparative manner, we injected construct HMMA223 to generate more selfdocking lines for $\varphi$ C31-mediated RMCE (Fig. 2C) into similar amounts of embryos on the 
same day and with the same injection needle to minimize variations in the injection procedure. Table 1 shows that no transgenic lines were obtained with the US or Italian strains, but were successfully obtained with the French AM strain with a transformation rate of $4.2 \%$. This demonstrates the higher usability of the AM strain for piggyBac germline transformation.

\section{Isolation of an enhancer/promoter region active during early embryonic development}

To direct gene expression specifically at early embryonic development, we identified the serendipity $\alpha$ (sry $\alpha$ ) gene by homology search in the $D$. suzukii genome database (www.spottedwingflybase.org) using the Dm_sry $\alpha$ sequence as query. The open reading frame of the Ds_sry $\alpha$ gene from the translation start codon to the stop codon is $1593 \mathrm{bp}$ without introns. The gene has a 5 'UTR of $49 \mathrm{bp}$, which demarcates the transcription start site (Fig. 1A). The Ds_sry $\alpha$ coding sequence encodes a putative protein of 530 amino acids, which shares $86 \%$ identity to Dm_Sry $\alpha$ protein.

To validate the cellularization-specific expression of the isolated Ds_sry $\alpha$ gene, we performed whole mount in situ hybridization on different stage wild type embryos using a DIG-labelled antisense probe against the whole Ds_sry $\alpha$ ORF plus the 5' UTR. These in situ hybridizations detected expression only during blastoderm cellularization with no expression at earlier or later embryonic stages (Fig. 1C-E).

To identify the necessary upstream and downstream regulatory elements driving cellularization-specific gene expression, we compared the Ds_sry $\alpha$ sequence with the characterized counterpart in D. melanogaster [9]. To examine, whether the 300 bp upstream regulatory element plus the 49 bp 5'UTR drive cellularization-specific gene expression, we fused this $349 \mathrm{bp}$ enhancer/promoter fragment of the Ds_sry $\alpha$ gene to $t T A$ (Fig. 1B) and generated D. suzukii line 06_F5M2 [52] by piggyBac-based germline transformation. Embryos from this line were then tested by whole mount in situ hybridization for expression of $t T A$, which revealed the respective cellularization-specific expression pattern of $D$ s_sry $\alpha$ (Fig. 1F-H) indicating that the isolated promoter/enhancer element is suitable for stagespecific gene expression during early embryonic development.

\section{Spermatogenesis-specific driver for binary tet-off expression system}

Since direct expression of effector molecules potentially causing harm obstructs the generation of transgenic lines, we aim to establish the tet-off binary system in D. suzukii to develop transgenic improvements for SIT approaches. To examine this binary expression system, we used the Ds_ $\beta 2 t$ enhancer/ promoter [52] to generate construct HMMA389 (Fig. 2A). By piggyBac-based germline transformation, we obtained the spermatogenesis-specific driver line 389_F25M1 that expresses $t$ TA in the testes. The spermatogenesis-specific expression was 
confirmed by in situ hybridization and compared to the endogenous expression of $D s \_\beta 2 t$ (Fig. 2B-D).

\section{$\varphi \mathrm{C} 31-$ mediated site-specific germline transformation}

Modification and/or stabilization of transgenes generated by transposon-based vectors by sitespecific recombination have been demonstrated in D. melanogaster and Ceratitis capitata [39, 55]. To establish $\varphi \mathrm{C} 31$-based site-specific germline transformation by integration of a transgene construct into a single attPsite, we injected donor plasmid HMMA182 carrying an $E G F P$ transformation marker and the bacterial attachment sequence att $B$ along with helper plasmid HMMA098 expressing $\varphi \mathrm{C} 31$ integrase under the promoter of the $D s-h s p 70$ gene into pre-blastoderm embryos of the DsRed-marked transgenic embryonic driver line 06_F5M2 (attP\#1). This line was generated with construct HMMA006 [52], which harbours in addition to the early embryonic tTA-driver also an attP site (Figs. 1B, 3A). Out of 250 injected embryos 110 hatched and 40 fertile $G_{0}$ crosses gave rise to four independent integrations (Additional File 2), which were identified by showing both red and green fluorescent markers (Fig. 3A'-A'"), resulting in a site-specific transformation efficiency of $10 \%$.

\section{$\varphi$ C31-mediated recombination mediated cassette exchange}

To examine a docking line with two attP sites in opposite orientation of a DsRed-based transformation marker for establishment of RMCE in D. suzukii, we used the docking line 185_F3F1 (RMCE\#1), which resulted from piggyBac-mediated integration of vector HMMA185 (Fig. 3B) into the AM strain (Additional files 1 and 2). In this line, we confirmed the presence of the two attP sites by sequencing. To see whether the $\varphi \mathrm{C} 31-$ based RMCE works in $D$. suzukii, we co-injected into this line plasmid HMMA336 having two attB recombination sites in opposite orientation flanking an EGFP-based transformation marker and the transgene of interest (an effector to drive Cas 9 expression under the control of the binary expression system tet-off) along with the helper plasmid HMMA098 (Fig.3B). We obtained $71 \mathrm{G}_{0}$ fertile crosses, of which eight gave rise to $\mathrm{F}_{1}$ progeny that showed EGFP and absence of DsRed fluorescence (Fig. 3B'-B'") indicating an RMCE rate of 11,3\%. RMCE line 336_F3F2 was then used to verify the faithful double recombination event by PCR and sequencing of the resulting hybrid att $L$ and attR sites (Fig. 3B).

\section{Isolation of an enhancer/promoter region active during oogenesis and in the germline to generate self-docking lines for $\varphi \mathrm{C} 31$-mediated RMCE}

To improve $\varphi \mathrm{C} 31$-mediated RMCE further, we wanted to establish self-docking lines (Fig.

4) that express $\varphi \mathrm{C} 31$ integrase maternally in addition to carrying two attPrecombination sites. In this respect, we identified the Ds_nanos gene by homology search in the D. suzukiigenome database (www.spottedwingflybase.org) using the Dm_nanos sequence as query. The open 
reading frame of the Ds_nanos gene from the translation start codon to the stop codon is 2433 bp, which is interrupted by three introns. The gene has a 5'UTR of $236 \mathrm{bp}$, which demarcates the transcription start site and a 3' UTR of 878 bp (Fig. 4A). To validate the oogenesis- and germline-specific gene expression of the isolated Ds_nanos gene, we performed whole mount in situ hybridization on ovaries using DIG-labelled antisense probes against the Ds_nanos 3'UTR and $103 \mathrm{bp}$ of exon IV. These in situ hybridizations detected expression in ovarian nurse cells (Fig. 4B).

To identify the necessary upstream and downstream regulatory elements driving oogenesisspecific gene expression, we compared the Ds_nanos sequence with the characterized counterpart in $D$. melanogaster. To examine, whether the $2 \mathrm{~Kb}$ enhancer/promoter region including the 5'UTR drives oogenesis-specific gene expression, we fused this $2 \mathrm{~Kb}$ enhancer/promoter fragment of the Ds_nanos gene to the coding region of $\varphi$ C31 integrase (Fig. 4C) and generated D. suzukii lines 223_F7M1 and 223_M3M2 by piggyBac-based germline transformation of the AM strain (Additional files 1 and 2). In addition, two more self-docking lines were generated in the comparative approach to evaluate the different $D$. suzukii strains (Table 1). Ovaries from line 223_M3M2 were then tested by whole mount in situ hybridization for expression of $\varphi C 31$ integrase, which revealed the respective nurse cellspecific expression in the ovaries (Fig. 4D) resembling Ds_nanos expression, which indicates that the isolated promoter/enhancer element is suitable for maternal gene expression.

\section{Discussion}

The discovery that exogenous DNA can be stably introduced into the germline of living organisms which can then be stably inherited by the offspring has tremendously contributed to the advancement of biological and biomedical research and in particular functional genetic studies [15, 19, 30, 31]. The road for insect genetic engineering has been well paved by geneticists working with the model organism $D$. melanogaster. Genetic screens in $D$. melanogaster using $P$-element based transformation vectors to perform insertional mutagenesis, enhancer- and gene-traps, as well as ectopic or overexpression studies provided an enormous contribution to our understanding of gene function [56-58]. Unfortunately, the $P$-element is not functional in other organisms due to the requirement of host-specific factors [59]. Transformation vectors based on the lepidopteran transposable element piggyBac have been used to engineer many insects $[20,22,25,26]$ and encouraged the establishment of new insect model systems such as Tribolium castaneum [60, 61].

The invasive fruit pest, D. suzukii, had been successfully transformed using piggyBac-based vectors $[25,54,62]$. We have used three different lab strains of $D$. suzukii from Italy, USA, and France. After many attempts to generate transgenic $D$ suzukii using piggyBac germline transformation with different constructs by microinjection into the Italian strain, we obtained only one transgenic line, 06_F5M2, with a low transformation efficiency of $1.6 \%$ and failed 
to obtain any transgenic flies from the US strain. When we obtained the French strain AM (which was kindly provided to us by N. Gompel, Munich), we managed to get reliably transgenics with varying efficiency. Based on these observations and the comparative examination of these three strains (Table 1), we recommend the AM strain for piggyBac germline transformation.

Due to the random integration of transposon-based transformation vectors and the limited size of cargo they can carry, we decided to extend the toolkit for D. suzukiitransformation by firmly establishing a site-specific transformation technology. Recombinase-based site-specific germline transformation such as (Cre/lox, flp/FRT and $\varphi \mathrm{C} 31$ attP/attB) had been established in many model and non-model insects and shown to overcome the shortcomings of transposon-based germline transformation [40]. The Cre/lox Recombinase Mediated Cassette Exchange has recently been established for the cherry vinegar fly D. suzukii [63]. In this study, we demonstrate the feasibility of using the $\varphi C 31$ integrase system to integrate a construct in a single attP landing site. This approach had previously been established for $D$. melanogaster and the Mediterranean fruit fly Ceratitis capitata, where it was used to modify transgenic lines generated by random transposon-based vectors and to stabilize the transgene by subsequent deletion of one of the inverted repeats required for transposition $[39,55]$. In addition, we have generated a docking line with two attP sites in opposite orientations and show that $\varphi \mathrm{C} 31$ mediated RMCE works in D suzukii. The use of an endogenous source of $\varphi \mathrm{C} 31$ integrase by expression from a germline specific enhancer/promoter had been shown to increase the efficiency of $\varphi \mathrm{C} 31$-mediated integration and RMCE $[42,43]$. In this regard, we set to generate self-docking lines that express $\varphi C 31$ integrase maternally. We isolated the endogenous Ds-nanos gene (Fig. 4) in order to use the upstream enhancer/promoter and the downstream 3'UTR for directing the expression of $\varphi C 31$ integrase to the nurse cells for maternal contribution to the early embryo. By random piggyBacgermline transformation, we generated four transgenic lines with a DsRed body marker and the $\varphi C 31$ integrase cassette flanked by attP sites.

To be able to conditionally drive expression of effector genes in a tissue- or stage-specific manner, a suppressible or inducible binary expression system is required. This has been successfully exploited to develop biotechnological pest control strategies such as early embryonic lethality or female-specific embryonic lethality [9-14]. To develop such transgenic pest control strategies for the invasive pest $D$. suzukii, we identified a gene that is active during early embryonic stages $\left(D s \_s r y \alpha\right)$ and a gene that is spermatogenesis-specific ( $\left.D s \_\beta 2 t\right)$ [52]. $350 \mathrm{bp}$ upstream regulatory sequence of the $D$ s_sry $\alpha$ gene were identified to be sufficient to drive the expression of $t T A$ specifically during cellularization similar to the endogenous gene. This driver line will be usable to generate reproductive sterility or sexing lines by driving expression of pro-apoptotic genes as previously described for several tephritid fruit pests [10$12,14]$. Such systems will be very important to establish SIT programs for the control of this 
invasive pest species. In addition, we were able to generate a spermatogenesis specific driver line using the promoter of the $D s \_\beta 2 t$ gene described previously [52].

\section{Conclusion}

By comparing different $D$. suzukii strains for their usability for piggyBac-based germline transformation, we could clearly identify the AM strain derived from the French Alps as the most suitable one. In addition, we demonstrated that $\varphi \mathrm{C} 31$-based site-specific integration and RMCE can be used routinely in the cherry vinegar fly, D. suzukii, and generated four self-docking lines for RMCE. The $\varphi \mathrm{C} 31$-based integration will facilitate efficient integration of larger transgenic constructs and allow for the modification and stabilization of previously generated transgenic lines that carry at least one attP site in the transgene construction.

\section{Methods}

\section{Drosophila suzukii strains}

All fly experiments were performed in our well-equipped safety level one (S1) laboratory, which is certified for generating and using genetically modified insects. Wild type D. suzukii from Italy, USA (both kindly provided by Prof. Marc F. Schetelig), and French Alps (Prof. Dr. Nicolas Gompel) as well as the generated transgenic flies were reared on standard Drosophila food and kept at $25^{\circ} \mathrm{C}$ throughout this study.

\section{Nucleic acid isolation}

Genomic DNA isolation was done from a mix of adult males and females using NucleoSpin ${ }^{\circledR}$ DNA Insect (Macherey-Nagel) according to the manufacturer instructions. Total RNA was isolated from 0-24 hours embryos enriched for 0-4 hours stages using ZR Tissue \& Insect RNA MicroPrep (Zymo Research Europe, 79110 Freiburg) according to manufacturer instructions.

All PCR amplifications during the course of this study were performed using Phusion DNA polymerase and Phusion-HF buffer (New England Biolabs GmbH, D-65926 Frankfurt am Main). A list of the used primers is provided in Additional file 3. Plasmid min-preps and PCR products were purified using NucleoSpin ${ }^{\circledR}$ Plasmid and NucleoSpin ${ }^{\circledR}$ Gel and PCR Cleanup kits (Macherey-Nagel GmbH \& Co., 52355 Dueren, Germany), respectively. NucleoSpin ${ }^{\circledR}$ Plasmid Transfection-grade (Macherey-Nagel) or QIAGEN Plasmid Plus Midi Kit (QIAGEN GmbH, 40724 Hilden, Germany) were used to prepare plasmids for germline transformation.

\section{Amplification of cDNA ends}

To isolate the 5'UTR and the 3'UTR of the early embryonic gene Ds_sry $\alpha$ and the maternal effect gene Ds_nanos, total RNA from 0-24 h old (enriched for 0-4h) D. suzukii embryos 
was isolated and $1.3 \mu \mathrm{g}$ were used to generate 5' RACE-ready cDNA or 3'RACE-ready cDNA using SMARTer ${ }^{\mathrm{TM}}$ RACE cDNA amplification kit (Takara Bio Europe SAS, 78100 Saint-Germain-en-Laye, France) according to manufacturer instructions.

The 5'UTR of Ds_sry $\alpha$ and Ds_nanos were recovered by RACE PCR using gene specific primers HM\#34 and HM\#76, respectively, along with the universal primer (UPM) provided with the kit using Advantage 2 DNA polymerase (Takara) with the following program: $94^{\circ} \mathrm{C}$ $2 \mathrm{~min},\left(94^{\circ} \mathrm{C} 30 \mathrm{sec}, 72^{\circ} \mathrm{C} 3 \mathrm{~min}\right) 5 \mathrm{X},\left(94^{\circ} \mathrm{C} 30 \mathrm{sec}, 70^{\circ} \mathrm{C} 30 \mathrm{sec}, 72^{\circ} \mathrm{C} 3 \mathrm{~min}\right) 5 \mathrm{X},\left(94^{\circ} \mathrm{C} 30\right.$ sec, $\left.68^{\circ} \mathrm{C} 30 \mathrm{sec}, 72^{\circ} \mathrm{C} 3 \mathrm{~min}\right) 30 \mathrm{X}$. A single prominent band for each gene was recovered, purified, cloned into pCRII (Thermo Fisher Scientific) to generate pCRII_sryo_5'UTR (HMMA001) and pCRII_nos_5UTR (HMMA012), and sequenced using standard M13 primers.

To recover the 3'UTR of Ds_sry $\alpha$ and Ds_nanos, the gene specific primers HM\#42 and HM\#77, respectively, along with UPM provided with the kit using Advantage2 DNA polymerase (Takara) were used with the following program: $94^{\circ} \mathrm{C} 2 \mathrm{~min},\left(94^{\circ} \mathrm{C} 30 \mathrm{sec}, 72^{\circ} \mathrm{C}\right.$ $3 \mathrm{~min}) 5 \mathrm{X},\left(94^{\circ} \mathrm{C} 30 \mathrm{sec}, 70^{\circ} \mathrm{C} 30 \mathrm{sec}, 72^{\circ} \mathrm{C} 3 \mathrm{~min}\right) 5 \mathrm{X},\left(94^{\circ} \mathrm{C} 30 \mathrm{sec}, 68^{\circ} \mathrm{C} 30 \mathrm{sec}, 72^{\circ} \mathrm{C} 3\right.$ $\mathrm{min}) 30 \mathrm{X}$. A single prominent band for each gene was recovered, purified, cloned into pCRII (Thermo Fisher Scientific) to generate pCRII_srya_3UTR (HMMA002) and pCRII_nos_3UTR (HMMA013), and sequenced using standard M13 primer.

\section{Plasmids construction}

The plasmid HMMA020 was generated by PCR amplification of the coding sequence of $D$. suzukii sryo gene plus the 5'UTR using primer pair HM\#16/HM\#17 and advantage 2 DNA polymerase (Invitrogene) with program $98{ }^{\circ} \mathrm{C} 3$ ' followed by $\left[98{ }^{\circ} \mathrm{C} 30 ”, 55{ }^{\circ} \mathrm{C} 30,, 72{ }^{\circ} \mathrm{C}\right.$ 2'] $35 \mathrm{X}$ and cloned into the pCRII vector (Invitrogene).

To generate plasmid HMMA021 for in vitro synthesis of RNA probes, the $t T A$ coding sequence was excised from mfs\#1215[10] using EcoRV/BamHI and cloning into pCRII vector digested by the same enzymes.

To generate plasmid HMMA339 for in vitro synthesis of RNA probe against $\varphi C 31$ integrase mRNA, $800 \mathrm{bp}$ of the coding sequence was digested out from plasmid HMMA98 using SmaI/Not and cloned into pCRII plasmid digested by EcoRV/Not.

The plasmid FCMH01 was generated by PCR amplification of 800 bp of Cas 9 coding

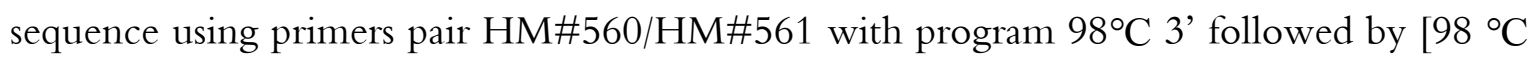
30 ”, $\left.64{ }^{\circ} \mathrm{C} 30^{\prime \prime}, 72{ }^{\circ} \mathrm{C} 30^{\prime \prime}\right] 5 \mathrm{X}$ [98 ${ }^{\circ} \mathrm{C} 30^{\prime \prime}, 72{ }^{\circ} \mathrm{C} 1$ '] 35X, digested by and cloned into Xhol/BamHI sites of pCRII vector.

To generate piggyBac transformation vector HMMA185 and HMMA186, first plasmid HMMA006 [52] was digested by $A s c$ to remove $s r y \alpha-t T A$, and the backbone was ligated to 
give rise to HMMA007. attP220 was PCR amplified from HMMA007 using primer pair HM\#368/HM369 and program $98^{\circ} \mathrm{C} 3$ 'followed by [98 'C 30”, $\left.58{ }^{\circ} \mathrm{C} 30^{\prime \prime}, 72{ }^{\circ} \mathrm{C} 20^{\prime \prime}\right] 5 \mathrm{X}$ $\left[98{ }^{\circ} \mathrm{C} 30^{\prime \prime}, 72^{\circ} \mathrm{C} 1^{\prime}\right] 35 \mathrm{X}$ and cloned into EcoRV cut site of HMMA007 to give rise to HMMA185. To generate HMMA186 the EcoRI/HpaI fragment PUb::nlsEGFP from mfs\#1213 [51] was cloned into the EcoRI/HpaI sites of HMMA185.

For the generation of piggyBac transformation vectors HMMA330 and HMMA331, first Gibson assembly was performed to clone EGFPSV4O and the $3 X P 3$ promoter into the piggy Bac backbone of HMMA007 digested by EcoRI to give rise to HMMA227, in which the EGFP gene was then replaced by DsRed.T3 from HMMA007 by AgeI/Not to give rise to HMMA228. Then the attP220 was PCR amplified from HMMA007 using primer pair HM\#131/HM\#117 with PCR program $98{ }^{\circ} \mathrm{C} 3$ 'followed by $\left[98^{\circ} \mathrm{C} 30^{\prime \prime}, 60{ }^{\circ} \mathrm{C} 30^{\prime \prime}, 72{ }^{\circ} \mathrm{C}\right.$ 20"] $35 \mathrm{X}$ and cloned into EcoRI site of HMMA227 and HMMA228 giving rise to HMMA304 and HMMA305, respectively. Finally, the AscI/AgeI fragments from mfs\#1213 and mfs\#1214 [51] containing the PUb promoter were cloned into Ascl/Agel sites of HMMA304 and HMMA305 to give rise to HMMA330 and HMMA331, respectively.

To generate the spermatogenesis specific driver construct HMMA389, $1 \mathrm{~kb}$ upstream region of $D$. suzukii Ds- $\beta 2 t$ gene including the 5'UTR was PCR amplified from genomic DNA of the wild type Italian strain using primer pair HM\#35/HM\#36 with program $98{ }^{\circ} \mathrm{C} 3^{\prime}\left[98^{\circ} \mathrm{C}\right.$ 30 ", $\left.61{ }^{\circ} \mathrm{C} 30^{\prime \prime}, 72{ }^{\circ} \mathrm{C} 30^{\prime \prime}\right] 5 \mathrm{X}$ [ $\left.98^{\circ} \mathrm{C} 30^{\prime \prime}, 67^{\circ} \mathrm{C} 30^{\prime \prime} 72^{\circ} \mathrm{C} 30^{\prime \prime}\right] 35 \mathrm{X}$ and cloned in NcoI/ XbaI sites of mfs\#1215 [10] giving rise to HMMA015. The Dm- $\beta 2 t$ UUTR was then PCR amplified from gDNA of wild type $D$. melanogaster strain $\mathrm{OreR}$ using primer pair HM\#706/HM\#707 with program $98{ }^{\circ} \mathrm{C} 3$ ' [98 $\left.{ }^{\circ} \mathrm{C} 30 ", 63{ }^{\circ} \mathrm{C} 30^{\prime \prime}, 72{ }^{\circ} \mathrm{C} 20^{\prime \prime}\right] 5 \mathrm{X}\left[98^{\circ} \mathrm{C}\right.$ $\left.30 ", 70{ }^{\circ} \mathrm{C} 30^{\prime \prime} 72{ }^{\circ} \mathrm{C} 20^{\prime \prime}\right] 35 \mathrm{X}$ and cloned into HMMA015 to give rise to HMMA253. Finally, the Asc fragment from HMMA253 was cloned into the Asc site of the transformation vector HMMA331.

To generate attBintegration vector HMMA182 which can be used to integrate a plasmid into single attP site, the 5-piggyBac region was PCR amplified from plasmid HMMA006 using primer pair T7/mfs \#370, with program $98{ }^{\circ} \mathrm{C} 3$ ' [98 'C 30", $51{ }^{\circ} \mathrm{C} 30$ ”, $\left.72{ }^{\circ} \mathrm{C} 20^{\prime \prime}\right] 40 \mathrm{X}$ digested by EcoRV and cloned into the blunted BamHI site of HMMA172, giving rise to HMMA181. Then the EcoRI/ApaI fragment containing the PUb::nlsEGFP was excised mfs\#1213 [51] and cloned into EcoRI/ApaI of HMMA181.

To generate the helper plasmid HMMA098, the coding sequence of $\varphi \mathrm{C} 31$ was PCR amplified from plasmid mfs\#1289 [39] using primers pair MK153/HM\#123 with program 98 ${ }^{\circ} \mathrm{C} 3$ ' [98 'C 30", $72{ }^{\circ} \mathrm{C} 1^{\prime}$ '20'] 35X. The reverse primer introduces the SV40 nuclear localization sequence at the $\mathrm{C}$-terminus, which can improve the efficiency of $\varphi \mathrm{C} 31$ integrase [64]. A second round of PCR using primer pair MK153/HM\#203 was used to amplify $\varphi C 31 n l s$ using $1 \mu \mathrm{l}$ of the first PCR reaction as a template with program $98^{\circ} \mathrm{C} 3$ ' [98 ${ }^{\circ} \mathrm{C} 30^{\prime \prime}$, 
$67{ }^{\circ} \mathrm{C} 30^{\prime \prime}, 72{ }^{\circ} \mathrm{C} 1$ '] $5 \mathrm{X}\left[98^{\circ} \mathrm{C} 30^{\prime \prime}, 72{ }^{\circ} \mathrm{C} 1\right.$ ' 20”'] 35X and clone into HMMA051 NcoI/Not replacing the piggyBac transposase coding sequence and giving rise to HMMA098. The piggyBac helper HMMA051 was generated by cloning the SV4O 3'UTR digested from CH\#705 by HindII/Not into HMMA050 HindIII/NotI. sites. The latter was made by PCR amplification of $D s-h s p 70$ promoter [52] from gDNA using primer pair HM73/HM\#74 and program $98{ }^{\circ} \mathrm{C} 3$ 'followed by [98 $\left.{ }^{\circ} \mathrm{C} 30^{\prime}, 58^{\circ} \mathrm{C} 30^{\prime \prime}, 72{ }^{\circ} \mathrm{C} 30^{\prime \prime}\right] 5 \mathrm{X}\left[98^{\circ} \mathrm{C} 30^{\prime \prime}, 66^{\circ} \mathrm{C} 30^{\prime \prime}\right.$, $\left.72{ }^{\circ} \mathrm{C} 30^{\prime}\right] 35 \mathrm{X}$ and cloning into EcoRI site of HMMA049, which was generated by cloning the piggyBac transposase coding sequence excised from MK004 [23] by EcoRI/Not into the shuttle vector pSLaf1180af [65].

To generate $\varphi \mathrm{C} 31$ integrase based RMCE donor plasmids, HMMA253 and HMMA254, the annealed oligos HM\#101/HM\#337 generating the bacterial attachment site attB were cloned into SpeI site of pCRII vector (Invitrogene) giving rise to HMMA172. The gypsy insulators were digested out using Spel/EcoRI from a fragment amplified from mfs\#1213[51] using primer pair HM\#469/HM\#470 with program $98{ }^{\circ} \mathrm{C} 3$ 'followed by $\left[98^{\circ} \mathrm{C} 30\right.$ ", $70{ }^{\circ} \mathrm{C} 30^{\prime \prime}$, $72{ }^{\circ} \mathrm{C} 2$ '] $35 \mathrm{X}$ and cloned into the cut site of HMMA172 to give rise to HMMA189. The EcoRI/Not fragments PUb::nlsEGFP and PUb::DsRed.T3 were excised from HMMA186 and HMMA185, respectively, and cloned into HMMA189 to give rise to HMMA190 and HMMA191, respectively. Finally, SV40 was PCR amplified from HMMA007 using primer pair HM\#179/HM\#124 and program $98^{\circ} \mathrm{C} 3$ 'followed by [98 'C 30", $\left.62{ }^{\circ} \mathrm{C} 30^{\prime \prime}, 72^{\circ} \mathrm{C} 20^{\prime \prime}\right]$ $5 \mathrm{X}$ [98 $\left.{ }^{\circ} \mathrm{C} 30^{\prime \prime}, 68{ }^{\circ} \mathrm{C} 30^{\prime \prime}, 72{ }^{\circ} \mathrm{C} 20^{\prime \prime}\right] 35 \mathrm{X}$ and cloned along with annealed oligos HM\#101/HM\#108 into HMMA190 and HMMA191 Not/ XbaI-blunted.

To generate HMMA336, for $\varphi \mathrm{C} 31-\mathrm{RMCE}$, the tetracycline responsive element TRE along with the $P$-element basal promoter was PCR amplified from $\mathrm{CH} 727$ [9] using primers pair HM\#584/ CH6R [9] with PCR program $98^{\circ} \mathrm{C} 3$ 'followed by $\left[98^{\circ} \mathrm{C} 30^{\prime}, 69^{\circ} \mathrm{C} 30^{\prime}, 72^{\circ} \mathrm{C}\right.$ 30"' 35X and cloned into EcoRI/ ClaI sites of HMMA56 [52] replacing the hsp70 promoter giving rise to HMMA317 then the $A s C$ fragment containing Cas 9 fused to the TREp and the SV403'UTR was clone into AscI site of HMMA253

To generate self-docking transformation plasmid HMMA223 the Asc fragment containing nosE/P- $\varphi \mathrm{C} 31-$ nos was excised from the shuttle vector HMMA221 and cloned into Asc site of HMMA185. HMMA221 was generated by replacement of Cas 9 coding sequence in plasmid HMMA167 by $\varphi$ C31 integrase CDS. To make HMMA167, first the 3UTR of Dsnanos was PCR amplified from HMMA013 using primer pair HM\#94/HM95 with program $98{ }^{\circ} \mathrm{C} 3$ 'followed by $\left[98^{\circ} \mathrm{C} 30^{\prime}, 66^{\circ} \mathrm{C} 30 ”, 72{ }^{\circ} \mathrm{C} 30\right] 5 \mathrm{X}\left[98{ }^{\circ} \mathrm{C} 30 ”, 72{ }^{\circ} \mathrm{C} 1\right.$ '] 35X and cloned into the shuttle vector pSLaf1180af [65] XbaI/AflI sites giving rise to HMMA062. Then Cas 9 CDS was excised from HMMA056 [52] and cloned into ClaI/XbaI sites of HMMA062 giving rise to HMMA165. Then the palindromic (self-complementary) oligo HM\#102 was annealed to itself to introduce the 2X BbsI recognition site and cloned into the 
ClaI site of HMMA165 to give rise to HMMA166. Finally, a $2 \mathrm{~Kb}$ upstream regulatory region of Ds-nanos gene including the 5'UTR was PCR amplified from gDNA using primer pair HM\#345/HM\#113 and program $98{ }^{\circ} \mathrm{C} 3$ 'followed by [98 ${ }^{\circ} \mathrm{C} 30 ”, 72{ }^{\circ} \mathrm{C} 1$ ' 30”] 35X and cloned into HMMA166 BbsI site by golden gate resulting in HMMA167.

\section{Germline transformation}

All piggyBac germline transformation experiments were performed using transformation vector and helper plasmid MK006 [23] at a final concentration of $500 \mathrm{ng} / \mu \mathrm{L}$ and $200 \mathrm{ng} / \mu \mathrm{L}$ respectively. For $\varphi \mathrm{C} 31$-mediated site-specific transformation and $\varphi \mathrm{C} 31$-mediated $\mathrm{RMCE}$, the donor vectors were injected along with the helper plasmid HMMA098 at a concentration of $500 \mathrm{ng} / \mu \mathrm{L}$ and $300 \mathrm{ng} / \mu \mathrm{L}$, respectively. The materials and the procedure of germline transformation were as described previously [23, 52]. Emerged $\mathrm{G}_{0}$ flies were crossed individually to three wild type flies of the opposite sex.

\section{Generation of RNA probes}

To generate DIG-labelled antisense RNA probes for in situ hybridization against Ds_sryo, Ds_nanos, $t T A$, Cas 9 , or $\varphi C 31$ integrase, DNA templates for in vitro transcription were prepared by restriction enzyme linearization of pCRII vectors containing either the whole gene pCRII_Ds-sry $\alpha$ (HMMA020), the 3'RACE fragment pCRII_Ds-nos_3UTR (HMMA013), the coding sequence pCRII_tTA (HMMA021), or 800 bp of the coding sequence of in case of pCRII_Cas9(FCMH01) and pCRII_ $\varphi$ C31 (HMMA399) using XhoI, BamHI, NotI, NotI, or EcoRI, respectively. The antisense RNA labelling reaction was done using the DIG-labelling kit (Thermo Fisher Scientific) according to manufacturer instructions using $1 \mu \mathrm{g}$ of DNA as template in a total reaction mix of $20 \mu \mathrm{L}$. The reaction was allowed to proceed for $3 \mathrm{~h}$ at $37^{\circ} \mathrm{C}$ followed by Turbo DNaseI treatment (Thermo Fisher Scientific) for $30 \mathrm{~min}$ to remove template DNA. $2 \mu \mathrm{L}$ of $0.2 \mathrm{M}$ EDTA were used to inactivate the reaction. The probes were then ethanol precipitated and resuspended in $100 \mu \mathrm{L}$ RNA resuspension buffer $\left(5: 3: 2 \mathrm{H}_{2} \mathrm{O}: 20 \mathrm{X}\right.$ SSC: formaldehyde) and stored at $-80^{\circ} \mathrm{C}$.

\section{Testes, ovary, and embryo whole mount in situ hybridization}

Testes from 3-5 days old males from wild type $D$. suzukii, spermatogenesis specific driver line 389_M25M1, or progeny of the cross of the driver 389_M25M1 to the responder line 366_F3F1 were dissected in ice cold 1X phosphate buffered saline (PBS). Fixation and in situ hybridization were performed according to protocol by Lecuyer [66]. Anti-sense DIG labelled RNA probe against $t T A$ was used to detect the expression driven by the $D s-\beta 2 t E / P$. The Cas 9 anti-sense RNA probe was used to detect the expression of Cas 9 in the progenies arising from the cross testing the tet-off system. Anti-sense and sense probes previously described [52] were used as control. 
To confirm the expression of the isolated Ds-nanos gene and the $\varphi C 31$ integrase driven by the regulatory regions of Ds-nanos in the ovaries of $D$. suzukii wild type flies and the transgenic self-docking line 223_F7M1, respectively, we collected 3-5 days old female flies and dissected the ovaries in ice-cold $1 \mathrm{X}$ PBS. The fixation and the in situ hybridization were performed as described [66].

To confirm the endogenous cellularization-specific expression of Ds_sry $\alpha$ in wild type embryos. and whether the $349 \mathrm{bp}$ of its upstream regulatory region including the 5'UTR are enough to drive expression of $t T A$ in the transgenic driver line 06_F5M2 in a similar pattern, we performed embryo whole mount in situ hybridization using respective anti-sense DIGlabelled RNA probes in $0-24 \mathrm{~h}$ old embryos. Fixation and in situ hybridization were performed according to Lecuyer [66].

\section{Microscopy}

To observe and image testes, ovaries, and embryos, Zeiss Imager.Z2 equipped with two cameras, Axiocam 506 mono and Axiocam 305 colour (Zeiss, 73447 Oberkochen, Germany) was used. Images were taken using Axiocam 305 with bright field or DIC settings.

Screening for transgenic flies and fluorescence imaging were performed using Leica M205 FA fluorescence stereomicroscope equipped with camera Q imaging Micropublisher 5.0 RTV (Leica Mikrosysteme Vertrieb Gmb, Wetzlar, 35578 Germany). Transgenic flies were screened using filter sets RFP (excitation: ET546/10, emission: ET605/70) or GFP-LP (excitation: ET480/40, emission: ET510 LP), respectively, and imaged using cold light (Fig. 3A', B'), filter sets RFP (Fig. 3A", B"), or EYFP (excitation: ET500/20, emission: ET535/30; Fig. 3A'", B"').

\section{Declarations:}

Ethics approval and consent to participate: Not applicable.

Consent to publish: Not applicable.

\section{Availability of data and materials:}

All data generated or analysed during this study are included in this published article and its supplementary information files.

\section{Competing interests:}

The authors declare that they have no competing interests. 


\section{Funding}

H.M.M.A was supported by the German Academic Exchange Service (DAAD), which had no role in the design of the study, the collection, analysis, and interpretation of data, or in writing the manuscript. Publication costs are funded by the Joint FAO/IAEA Division of Nuclear Techniques in Food and Agriculture, IAEA (CRP No.: D4.20.16) Vienna, Austria.

\section{Authors' Contributions:}

E.A.W and H.M.M.A. conceived and designed the study; H.M.M.A. isolated the genes, designed the constructs, and performed in situ hybridizations; H.M.M.A. and F.H. generated the transgenic lines; E.A.W and H.M.M.A. wrote the manuscript; H.M.M.A. prepared the figures; all authors read and approved the final manuscript.

\section{Acknowledgements}

We would like to thank Marc F. Schetelig (Justus-Liebig-University Giessen) for providing wild type Drosophila suzukii strains (Italian and US), Nicolas Gompel (Ludwig-MaximillianUniversity Munich) for the AM strain from the French Alps. This study was benefitted from discussions at International Atomic Energy Agency funded meetings for the Coordinated Research Project "Comparing Rearing Efficiency and Competitiveness of Sterile Male Strains Produced by Genetic, Transgenic or Symbiont-based Technologies. 


\section{References}

1. Hauser M. A historic account of the invasion of Drosophila suzukii (Matsumura) (Diptera: Drosophilidae) in the continental United States, with remarks on their identification. Pest Management Science. 2011;67:1352-7.

2. Walsh DB, Bolda MP, Goodhue RE, Dreves AJ, Lee J, Bruck DJ, et al. Drosophila suzukii (Diptera: Drosophilidae): Invasive Pest of Ripening Soft Fruit Expanding its Geographic Range and Damage Potential. Journal of Integrated Pest Management. 2011;2:G1-7.

3. Cini A, Ioriatti C, Anfora G. A review of the invasion of Drosophila suzukii in Europe and a draft research agenda for integrated pest management. B INSECTOL Journal ISSN: 1721-8861. 2012;65:12.

4. Mazzi D, Bravin E, Meraner M, Finger R, Kuske S. Economic Impact of the Introduction and Establishment of Drosophila suzukii on Sweet Cherry Production in Switzerland. Insects. 2017;8:18.

5. Haviland DR, Beers EH. Chemical Control Programs for $<$ I $>$ Drosophila suzukii $</$ I $>$ that Comply With International Limitations on Pesticide Residues for Exported Sweet Cherries. $\mathrm{j}$ integ pest manage. 2012;3:1-6.

6. Van Timmeren S, Isaacs R. Control of spotted wing drosophila, Drosophila suzukii, by specific insecticides and by conventional and organic crop protection programs. Crop Protection. 2013;54:126-33.

7. Knipling EF. Possibilities of Insect Control or Eradication Through the Use of Sexually Sterile Males1. Journal of Economic Entomology. 1955;48:459-62.

8. Heinrich JC, Scott MJ. A repressible female-specific lethal genetic system for making transgenic insect strains suitable for a sterile-release program. Proceedings of the National Academy of Sciences. 2000;97:8229-32.

9. Horn C, Wimmer EA. A transgene-based, embryo-specific lethality system for insect pest management. Nat Biotechnol. 2003;21:64-70.

10. Schetelig MF, Caceres C, Zacharopoulou A, Franz G, Wimmer EA. Conditional embryonic lethality to improve the sterile insect technique in Ceratitis capitata(Diptera: Tephritidae). BMC Biol. 2009;7:4.

11. Ogaugwu CE, Schetelig MF, Wimmer EA. Transgenic sexing system for Ceratitis capitata (Diptera: Tephritidae) based on female-specific embryonic lethality. Insect Biochemistry and Molecular Biology. 2013;43:1-8.

12. Yan $Y$, Scott MJ. A transgenic embryonic sexing system for the Australian sheep blow fly Lucilia cuprina. Sci Rep. 2015;5:16090. 
13. Concha C, Palavesam A, Guerrero FD, Sagel A, Li F, Osborne JA, et al. A transgenic male-only strain of the New World screwworm for an improved control program using the sterile insect technique. BMC Biol. 2016;14:72.

14. Schetelig MF, Handler AM. A transgenic embryonic sexing system for Anastrepha suspensa (Diptera: Tephritidae). Insect Biochemistry and Molecular Biology. 2012;42:790-5.

15. Woltjen K, Michael IP, Mohseni P, Desai R, Mileikovsky M, Hämäläinen R, et al. piggyBac transposition reprograms fibroblasts to induced pluripotent stem cells. Nature. 2009;458:766-70.

16. Wang W, Lin C, Lu D, Ning Z, Cox T, Melvin D, et al. Chromosomal transposition of PiggyBac in mouse embryonic stem cells. Proceedings of the National Academy of Sciences. 2008;105:9290-5.

17. Park MA, Jung HS, Slukvin I. Genetic Engineering of Human Pluripotent Stem Cells Using PiggyBac Transposon System. Current Protocols in Stem Cell Biology. 2018;47:e63.

18. Cary LC, Goebel M, Corsaro BG, Wang HG, Rosen E, Fraser MJ. Transposon mutagenesis of baculoviruses: analysis of Trichoplusia ni transposon IFP2 insertions within the FP-locus of nuclear polyhedrosis viruses. Virology. 1989;172:156-69.

19. Hacker U, Nystedt S, Barmchi MP, Horn C, Wimmer EA. piggyBac-based insertional mutagenesis in the presence of stably integrated $\mathrm{P}$ elements in Drosophila. Proceedings of the National Academy of Sciences. 2003;100:7720-5.

20. Handler AM. Use of the piggyBac transposon for germ-line transformation of insects. Insect Biochemistry and Molecular Biology. 2002;32:1211-20.

21. Handler AM, Ii RAH. Germline transformation of Drosophila melanogaster with the piggyBac transposon vector. Insect Molecular Biology. 1999;:9.

22. Handler AM, McCombs SD, Fraser MJ, Saul SH. The lepidopteran transposon vector, piggyBac, mediates germ-line transformation in the Mediterranean fruit fly. Proceedings of the National Academy of Sciences. 1998;95:7520-5.

23. Eckermann KN, Ahmed HMM, KaramiNejadRanjbar M, Dippel S, Ogaugwu CE, Kitzmann P, et al. Hyperactive piggyBac transposase improves transformation efficiency in diverse insect species. Insect Biochemistry and Molecular Biology. 2018;98:16-24.

24. Condon KC, Condon GC, Dafa'alla TH, Forrester OT, Phillips CE, Scaife S, et al. Germ-line transformation of the Mexican fruit fly. Insect Molecular Biology. 2007;16:573-80.

25. Schetelig MF, Handler AM. Germline transformation of the spotted wing drosophilid, Drosophila suzukii, with a piggyBac transposon vector. Genetica. 2013;141:189-93. 
26. Grossman GL, Rafferty CS, Clayton JR, Stevens TK, Mukabayire O, Benedict MQ. Germline transformation of the malaria vector, Anopheles gambiae, with the piggyBac transposable element. Insect Molecular Biology. 2001;10:597-604.

27. Lobo NF, Hua-Van A, Li X, Nolen BM, Fraser MJ. Germ line transformation of the yellow fever mosquito, Aedes aegypti, mediated by transpositional insertion of a piggyBac vector. Insect Molecular Biology. 2002;11:133-9.

28. Hediger M, Niessen M, Wimmer EA, Dübendorfer A, Bopp D. Genetic transformation of the housefly Musca domestica with the lepidopteran derived transposon piggyBac. Insect Molecular Biology. 2001;10:113-9.

29. Bonin CP, Mann RS. A piggyBac Transposon Gene Trap for the Analysis of Gene Expression and Function in Drosophila. Genetics. 2004;167:1801-11.

30. O’Brochta DA, Alford RT, Pilitt KL, Aluvihare CU, Harrell RA. piggyBac transposon remobilization and enhancer detection in Anopheles mosquitoes. Proceedings of the National Academy of Sciences. 2011;108:16339-44.

31. Gayle S, Pan Y, Landrette S, Xu T. piggyBac Insertional Mutagenesis Screen Identifies a Role for Nuclear RHOA in Human ES Cell Differentiation. Stem Cell Reports. 2015;4:926-38.

32. Henikoff S. Position effect and related phenomena. Current Opinion in Genetics \& Development. 1992;2:907-12.

33. Levis R, Hazelrigg T, Rubin GM. Effects of genomic position on the expression of transduced copies of the white gene of Drosophila. Science. 1985;229:558-61.

34. Siegal ML, Hartl DL. Transgene Coplacement and high efficiency site-specific recombination with the Cre/loxP system in Drosophila. Genetics. 1996;144:715-26.

35. Long D-P, Zhao A-C, Chen X-J, Zhang Y, Lu W-J, Guo Q, et al. FLP Recombinase-Mediated Site-Specific Recombination in Silkworm, Bombyx mori. PLoS ONE. 2012;7:e40150.

36. Turan S, Galla M, Ernst E, Qiao J, Voelkel C, Schiedlmeier B, et al. Recombinase-Mediated Cassette Exchange (RMCE): Traditional Concepts and Current Challenges. Journal of Molecular Biology. 2011;407:193-221.

37. Turan S, Bode J. Site-specific recombinases: from tag-and-target- to tag-and-exchange-based genomic modifications. The FASEB Journal. 2011. doi:10.1096/fj.11-186940.

38. Bode J, Schlake T, Iber M, Schübeler D, Seibler J, Snezhkov E, et al. The Transgeneticists Toolbox: Novel Methods for the Targeted Modification of Eukaryotic Genomes. Biological Chemistry. 2005;381:801-813. 
39. Schetelig MF, Scolari F, Handler AM, Kittelmann S, Gasperi G, Wimmer EA. Site-specific recombination for the modification of transgenic strains of the Mediterranean fruit fly Ceratitis capitata. Proceedings of the National Academy of Sciences. 2009;106:18171-6.

40. Wimmer EA. Insect transgenesis by site-specific recombination. Nat Methods. 2005;2:580-2.

41. Venken KJT, He Y, Hoskins RA, Bellen HJ. P[acman]: A BAC Transgenic Platform for Targeted Insertion of Large DNA Fragments in D. melanogaster. Science. 2006;314:1747-51.

42. Bischof J, Maeda RK, Hediger M, Karch F, Basler K. An optimized transgenesis system for Drosophila using germ-line-specific $\varphi$ C31 integrases. PNAS. 2007;104:3312-7.

43. Meredith JM, Underhill A, McArthur CC, Eggleston P. Next-Generation Site-Directed Transgenesis in the Malaria Vector Mosquito Anopheles gambiae: Self-Docking Strains Expressing Germline-Specific phiC31 Integrase. PLOS ONE. 2013;8:e59264.

44. Leulier F, Vidal S, Saigo K, Ueda R, Lemaitre B. Inducible Expression of Double-Stranded RNA Reveals a Role for dFADD in the Regulation of the Antibacterial Response in Drosophila Adults. Current Biology. 2002;12:996-1000.

45. Matsushima Y, Adán C, Garesse R, Kaguni LS. Functional Analysis by Inducible RNA Interference in Drosophila melanogaster. In: Leister D, Herrmann JM, editors. Mitochondria. Totowa, NJ: Humana Press; 2007. p. 207-17. doi:10.1007/978-1-59745-365-3_15.

46. Gossen M, Bujard H. Tight control of gene expression in mammalian cells by tetracyclineresponsive promoters. Proceedings of the National Academy of Sciences. 1992;89:5547-51.

47. Urlinger S, Baron U, Thellmann M, Hasan MT, Bujard H, Hillen W. Exploring the sequence space for tetracycline-dependent transcriptional activators: Novel mutations yield expanded range and sensitivity. Proceedings of the National Academy of Sciences. 2000;97:7963-8.

48. Zhou X, Vink M, Klaver B, Berkhout B, Das AT. Optimization of the Tet-On system for regulated gene expression through viral evolution. Gene Ther. 2006;13:1382-90.

49. Fu G, Condon KC, Epton MJ, Gong P, Jin L, Condon GC, et al. Female-specific insect lethality engineered using alternative splicing. Nat Biotechnol. 2007;25:353-7.

50. Catteruccia F, Benton JP, Crisanti A. An Anopheles transgenic sexing strain for vector control. Nat Biotechnol. 2005;23:1414-7.

51. Scolari F, Schetelig MF, Bertin S, Malacrida AR, Gasperi G, Wimmer EA. Fluorescent sperm marking to improve the fight against the pest insect Ceratitis capitata (Wiedemann; Diptera: Tephritidae). New Biotechnology. 2008;25:76-84. 
52. Ahmed HMM, Hildebrand L, Wimmer EA. Improvement and use of CRISPR/Cas9 to engineer a sperm-marking strain for the invasive fruit pest Drosophila suzukii. BMC Biotechnol. 2019;19:85.

53. Eckermann KN, Dippel S, KaramiNejadRanjbar M, Ahmed HM, Curril IM, Wimmer EA. Perspective on the combined use of an independent transgenic sexing and a multifactorial reproductive sterility system to avoid resistance development against transgenic Sterile Insect Technique approaches. BMC Genet. 2014;15 Suppl 2:S17.

54. Karageorgi M, Bräcker LB, Lebreton S, Minervino C, Cavey M, Siju KP, et al. Evolution of Multiple Sensory Systems Drives Novel Egg-Laying Behavior in the Fruit Pest Drosophila suzukii. Current Biology. 2017;27:847-53.

55. Handler AM, Zimowska GJ, Horn C. Post-integration stabilization of a transposon vector by terminal sequence deletion in Drosophila melanogaster. Nat Biotechnol. 2004;22:1150-4.

56. Liebl FLW, Werner KM, Sheng Q, Karr JE, McCabe BD, Featherstone DE. Genome-widePelement screen forDrosophila synaptogenesis mutants. J Neurobiol. 2006;66:332-47.

57. Bachmann A, Knust E. The Use of P-Element Transposons to Generate Transgenic Flies. In: Dahmann C, editor. Drosophila. Totowa, NJ: Humana Press; 2008. p. 61-77. doi:10.1007/978-159745-583-1_4.

58. Venken KJT, Bellen HJ. Emerging technologies for gene manipulation in Drosophila melanogaster. Nature Reviews Genetics. 2005;6:167-78.

59. Rio DC. Identification and purification of a Drosophila protein that binds to the terminal 31-basepair inverted repeats of the P transposable element. Proc Natl Acad Sci USA. 1988;:5.

60. Brown SJ, Shippy T, Miller S, Bolognesi R, Beeman RW, Lorenzen MD, et al. The red flour beetle, Tribolium castaneum (Coleoptera): a model for studies of development and pest biology. Cold Spring Harbor protocols. 2009;2009:pdb.emo126.

61. Berghammer AJ, Klingler M, Wimmer EA. A universal marker for transgenic insects. Nature. 1999;402:370-1.

62. Buchman A, Marshall JM, Ostrovski D, Yang T, Akbari OS. Synthetically engineered Medeagene drive system in the worldwide crop pest Drosophila suzukii. Proc Natl Acad Sci USA. 2018;115:472530 .

63. Schetelig MF, Yan Y, Zhao Y, Handler AM. Genomic targeting by recombinase-mediated cassette exchange in the spotted wing drosophila, Drosophila suzukii. Insect Molecular Biology. 2019;28:187-95. 
64. Andreas S, Schwenk F, Küter-Luks B, Faust N, Kühn R. Enhanced efficiency through nuclear localization signal fusion on phage PhiC31-integrase: activity comparison with Cre and FLPe recombinase in mammalian cells. Nucleic Acids Res. 2002;30:2299-306.

65. Horn C, Wimmer EA. A versatile vector set for animal transgenesis. Dev Gene Evol. 2000;210:630-7.

66. Lécuyer E. High Resolution Fluorescent In Situ Hybridization in Drosophila. In: Gerst JE, editor. RNA Detection and Visualization. Totowa, NJ: Humana Press; 2011. p. 31-47. doi:10.1007/978-161779-005-8_3. 
Table 1: Comparative piggyBac transformation efficiency in different $D$. suzukii strains

\begin{tabular}{llllll}
\hline $\begin{array}{l}\text { Origin of } D \text {. suzukii } \\
\text { strain }\end{array}$ & $\begin{array}{l}\text { No. of injected } \\
\text { embryos }\end{array}$ & $\begin{array}{l}\text { Hatched } \\
\text { larvae }\end{array}$ & Fertile & No. of & Transformation \\
\hline Italy & 400 & 190 & 35 & 0 & transgenics \\
France (AM) & 450 & 210 & 47 & 2 & 4.2 \\
USA & 430 & 240 & 50 & 0 & - \\
\hline
\end{tabular}




\section{Figures:}
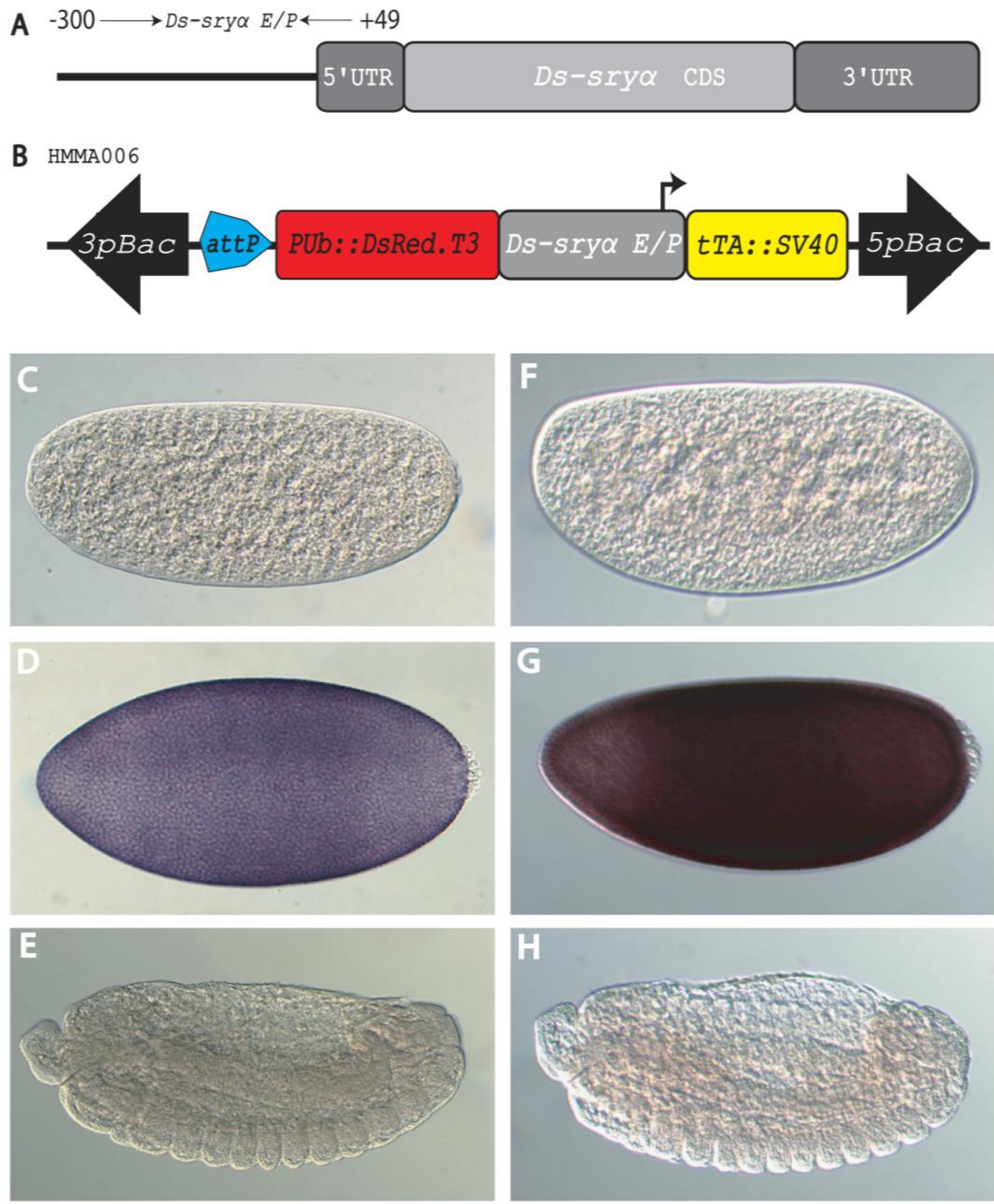

WT

srya 06-F5M2 (attP1)

tTA

Fig. 1. D. suzukii serendipity $\boldsymbol{\alpha}$ and the use of its promoter/enhancer for directed expression. (A) Schematic representation of the D. suzukii gene serendipity $\alpha$. (B) piggyBac-based transgenic construct HMMA006 [52] to drive $t T A$ during early embryonic development. (C-E) Whole mount in situ hybridisation to detect Ds_sry $\alpha$ expression in wildtype D. suzukii embryos. (F-H) Whole mount in situ hybridisation to detect $t T A$ expression in transgenic D. suzukii embryos of line 06_F5M2 (attP\#1) carrying construct HMMA006. (C, F) Syncytial blastoderm embryos before start of cellularization. $(\mathbf{D}, \mathbf{G})$ Syncytial blastoderm embryos during cellularization show expression of $\operatorname{sry} \alpha$ or $t T A$, respectively. (E, H) Germ band retracting embryos. 


\section{A HMMA389}

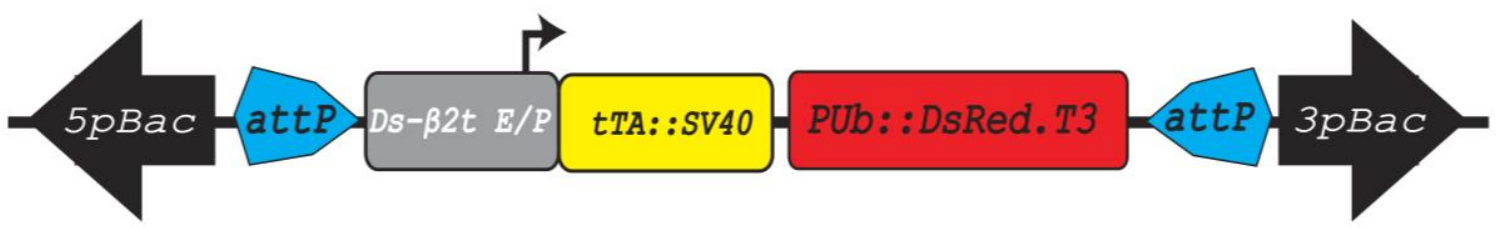

B

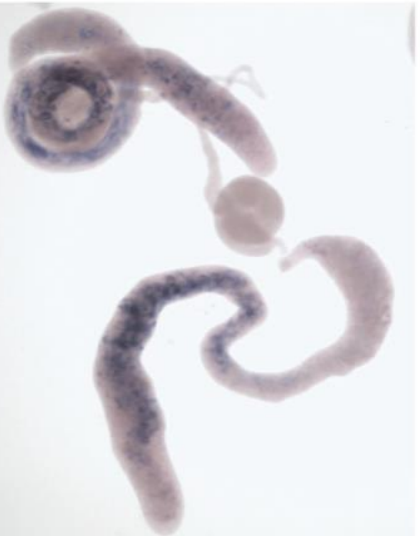

C tTA WT

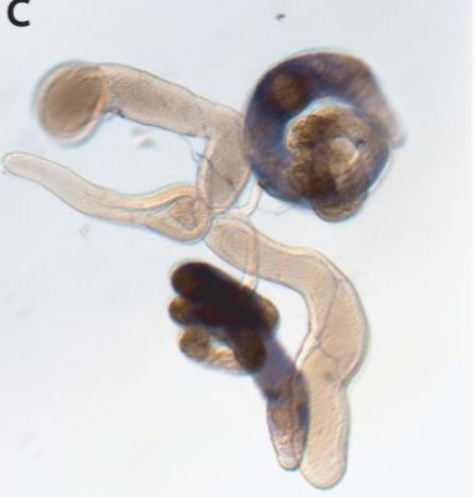

D

$\beta 2-t u b$ WT

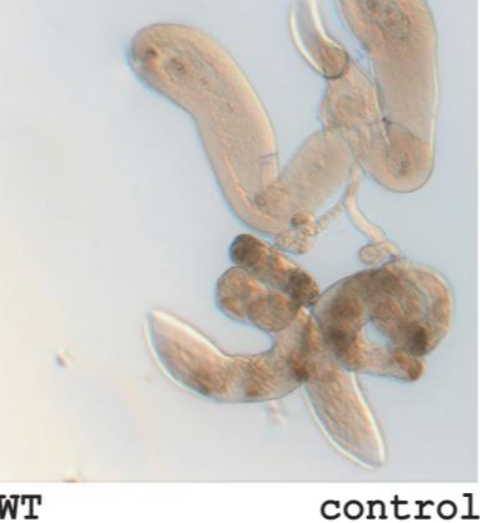

Fig. 2. Spermatogenesis-specific driver for binary tet-off expression system. (A) piggyBac-based transgenic construct HMMA389 to generate a testes-specific driver line carrying the $\beta 2 t$ promoter [52] fused to $t T A$. (B-D) Whole mount in situ hybridisation to detect gene expression in D. suzukii male reproductive organs. (B) Testes-specific $t T A$ expression driven by the $D s \_\beta 2 t$ promoter in line 389_F25M1. (C) $D s \_\beta 2 t$ expression in wildtype testes detected by an antisense probe. (D) Negative control using a $D s \_\beta 2 t$ sense probe on wildtype testes. 


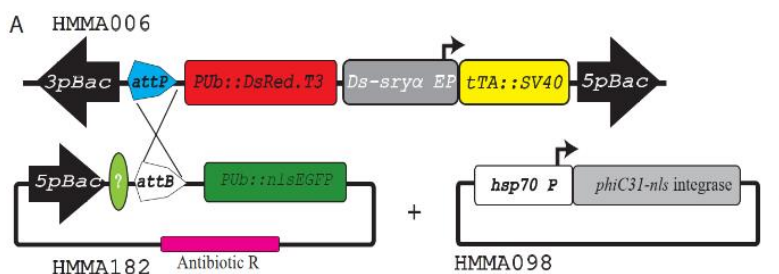

$A^{\prime}$

$06 \_$F5M2

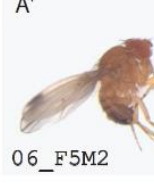

HMMA098
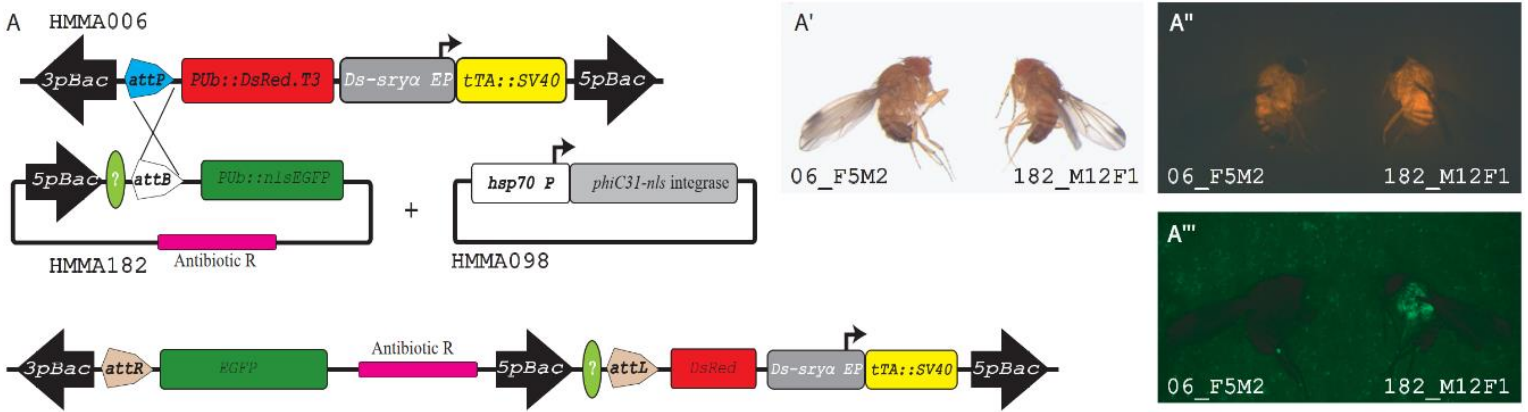

B HMMA185

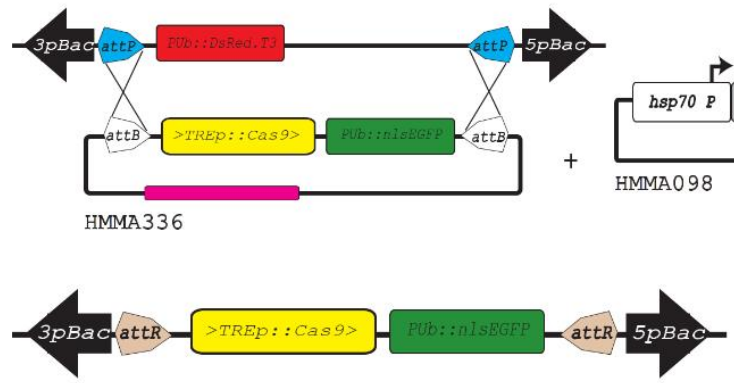
hiC31-nls integrase $B^{\prime}$

Fig. 3. $\varphi$ C31-mediated site-specific integration and RMCE. (A) Scheme for site-specific germline transformation. $D$. suzukii line 06_F5M2 [52] carries construct HMMA006 that contains an attPrecombination target sequence, which - in the presence of a helper plasmid providing $\varphi \mathrm{C} 31$ integrase (HMMA098) - is targeted by construct HMMA182 carrying the corresponding $a t t B$ recombination site to integrate the complete plasmid. The integration leads to a modification of the transgenic insert, which can be used for additional integration of transgenes (light green "?”) as well as transgene stabilization by removing part of the transgenic composition by piggyBac excision [39]. (A'-A'”') Integration can be detected by the addition of the EGFP marker. (B) RMCE to generate diverse transgenes at the same genomic position. D. suzukii line carrying construct HMMA185 is targeted by construct HMMA336 in the presence of a helper plasmid (HMMA098) providing $\varphi$ C31 integrase to exchange marker genes and integrate a specific cargo gene (TRE-Cas9). (B'-B'”') RMCE can be detected by the replacement of the DsRed marker with the EGFP marker. Images of a male fly of each indicated line are taken with cold light (A', B'), RFP filter (A', B'), or EYFP filter (A"', B'”). 
A

$-1764 \longrightarrow$ Ds-nos E/P $\longleftarrow+236 \quad \longrightarrow 878 \mathrm{bp} \longleftarrow$
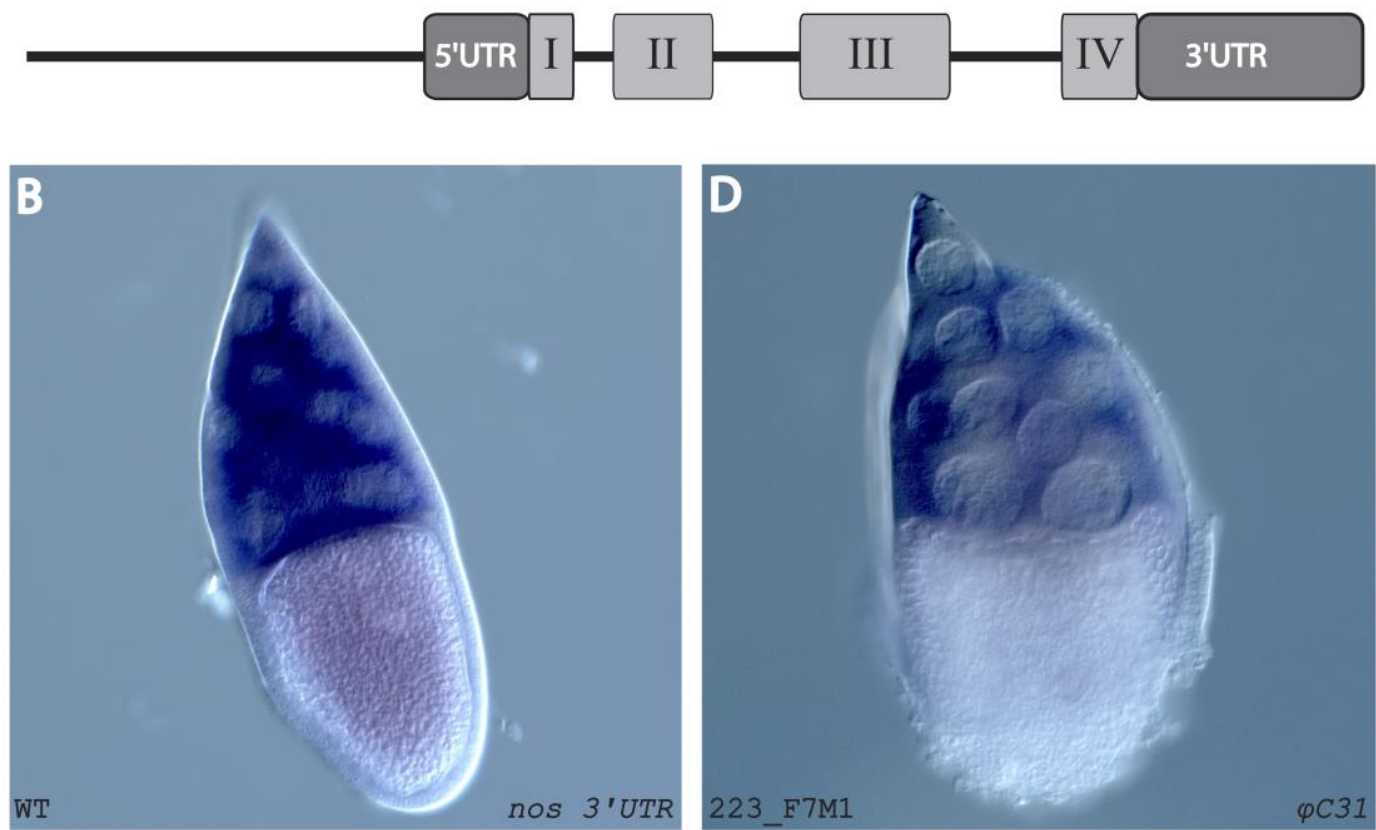

C

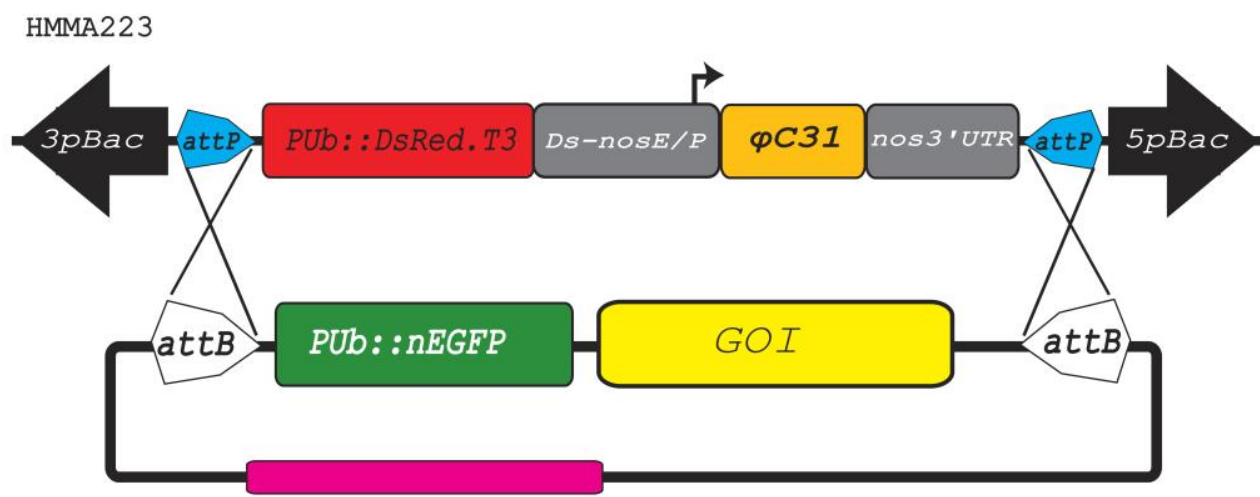

HMMA253

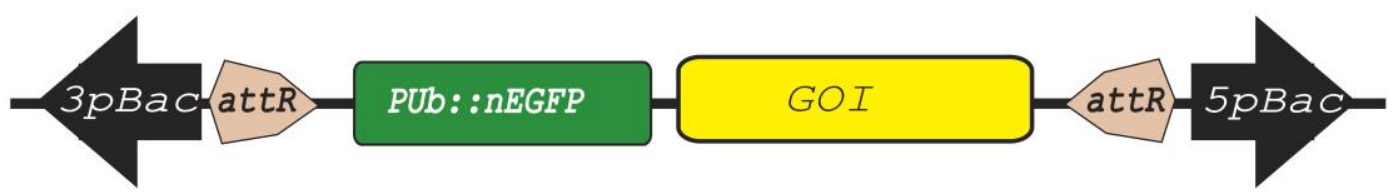

Fig. 4. D. suzukii nanos and the use of its promoter/enhancer for directed expression. (A) Schematic representation of the D. suzukii gene nanos $\square$ (B) Whole mount in situ hybridisation to detect nanos expression in wildtype D. suzukii ovaries. (C) piggyBac-based transgenic construct HMMA223 to generate $\varphi \mathrm{C} 31$ integrase RMCE self-docking lines. RMCE in a self-docking line, which provides both the recombination target sequences attPas well as the $\varphi \mathrm{C} 31$ integrase driven by the nanos promoter/enhancer providing maternal expression, will result in marker exchange as well as cargo gene (GOI) integration and removal of the integrase source. (D) Whole mount in situ hybridisation to detect $\varphi C 31$ integrase expression in transgenic D. suzukii ovaries carrying construct HMMA223. Expression of nanos or $\varphi C 31$ integrase, respectively, is detected in the nurse cells of the ovaries. 
Additional File 1

Supplementary Table 1: piggyBac transformation rates in $D$. suzukii AM strain

\begin{tabular}{lrrrrr}
\hline Construct & No injected embryos & Hatched larvae & Fertile crosses & No. transgenics & $\begin{array}{r}\text { Transformation } \\
\text { rate in } \%\end{array}$ \\
\hline HMMA389 & 350 & 185 & 40 & 1 & 2.5 \\
HMMA185 & 475 & 181 & 45 & $2^{*}$ & 4.4 \\
HMMA223 & 290 & 150 & 18 & 2 & 11 \\
\hline
\end{tabular}

* For one transgenic F1, no line could be established. 
Additional File 2.

\section{Supplementary Table 2: List of transgenic lines}

\begin{tabular}{|c|c|c|}
\hline Strategy & Construct & Transgenic lines \\
\hline \multirow{4}{*}{ 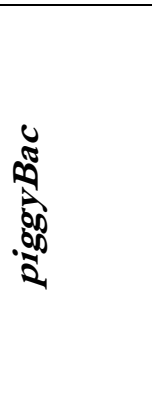 } & HMMA006 (embryonic driver, attP), Figs. 1B; 3A & 06_F5M2 (attP\#1) \\
\hline & HMMA389 (spermatogenesis driver), Fig. 2A & 389_F25M1 \\
\hline & HMMA185 ( $\varphi$ C31 RMCE docking), Fig. 3B & 185_F3F1 (RMCE\#1) \\
\hline & HMMA223 ( $\varphi$ C31 RMCE self-docking), Fig. 4C & $\begin{array}{l}\text { 223_M3M2 (RMCE-sd\#1) } \\
\text { 223_F7M1 (RMCE-sd\#2) } \\
\text { 223_F5F1 (RMCE-sd\#3) } \\
\text { 223_M10F1 (RMCE-sd\#4) }\end{array}$ \\
\hline 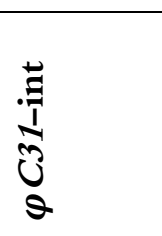 & $\begin{array}{l}\text { HMMA182 (single attB donor), Fig. 3A } \\
\text { Injected into 06_F5M2 (attP\#1) }\end{array}$ & $\begin{array}{l}\text { attP\#1_182_M12F1 } \\
\text { attP\#1_182_F8M1 } \\
\text { attP\#1_182_F15F1 } \\
\text { attP\#1_182_F25M1 }\end{array}$ \\
\hline$\sum_{\substack{1 \\
0}}^{0}$ & $\begin{array}{l}\text { HMMA336 (RMCE donor TREp:Cas9), Fig. 3B } \\
\text { Injected into 185_F3F1 (RMCE\#1) }\end{array}$ & $\begin{array}{l}\text { RMCE\#1_336_M1M1 } \\
\text { RMCE\#1_336_M17F1 } \\
\text { RMCE\#1_336_M21M1 } \\
\text { RMCE\#1_336_M32M1 } \\
\text { RMCE\#1_336_M33F1 } \\
\text { RMCE\#1_336_M34M3 } \\
\text { RMCE\#1_336_F12F1 } \\
\text { RMCE\#1_336_F3F2 }\end{array}$ \\
\hline
\end{tabular}


Additional File 3

\section{Supplementary Table 3: List of primers used}

\begin{tabular}{|c|c|c|}
\hline Code & Name & Sequence 5'- 3' \\
\hline HM\#16 & sryaR4 & TTGTGTGTCATGGATGTTCAATCTAATC \\
\hline HM\#17 & Srya5UTR_F1 & GTACTTAGTTGAAAAGTTCAGCTTTACCCG \\
\hline HM\#34 & Ds_srya_GSP3 & GGCATCCAGGCTAATGGTCCGCTCCAAGTG \\
\hline HM\#35 & B2t_Ncol_F1 & GCAACCATGGGATGCCAAGAGAGATGAGCAGG \\
\hline HM\#36 & B2t_Xbal_R3 & CGATTCTAGACATCTTAACCGACTGTCAAGGATC \\
\hline HM\#42 & srya_GSP_F & GCCTCTCTGGCTCCGATTCCCCCTAATG \\
\hline HM\#73 & hspEcoRIF2 & GCGAATTCTCCAGAAACTCAAACAGAAACC \\
\hline HM\#74 & hspEcoRIR & GCGAATTCTTGTGTGTTTGTGTTTGTGGATGCAG \\
\hline HM\#76 & nos_GSPR & GAGTCCTCCTCTTGCGTGGAATGCCG \\
\hline HM\#77 & nos_GSPF & GTACTGTCCCAAGAAGCCGATTATCAC \\
\hline HM\#94 & nos3UTRXbalF & GGTCTAGAGAACACATCCGGCAGGAGC \\
\hline HM\#95 & nos3UTRAflIIR & ATACTTAAGACTGAGCTCCAAGCAGTGGTATCAACGCAGAG \\
\hline HM\#101 & Spel-atBF & CTAGTGTGAGGTGGAGTACGCGCCCGGGGAGCCCAAGGGCACGCCCTGGCACCCGCAC \\
\hline HM\#102 & Bbsl-Bbsl & CGGTCTTCGCGAAGAC \\
\hline HM\#113 & Bbsl_nosR & GCGAAGACCCATATGGCGAAAGTCCGGCTCGAAAGTTACC \\
\hline HM\#117 & HM_Pub_R & CATTGGAATCTCTGTCGCTGCGTTCCG \\
\hline HM\#123 & phi_nls_R & CTAGACCTTCCGCTTCTTCTTTGGGGCCGCCGCTACGTCTTCCGTGCCGTCCTG \\
\hline HM\#124 & SV40_Spel_R & CACACTAGTGATACATTGATGAGTTTGGACAAACCACAAC \\
\hline HM\#131 & PLF2 & GTCAAAATGACGCATGATTATCTTTTACG \\
\hline HM\#179 & DsRed_End & CGAGGGCCGCCACCACCTGTTCCTG \\
\hline HM\#203 & Notl_phiC31_R & TCGCGGCCGCCTAGACCTTCCGCTTCTTCTTTGG \\
\hline HM\#337 & Spel_attB_R & CTAGGTGCGGGTGCCAGGGCGTGCCCTTGGGCTCCCCGGGCGCGTACTCCACCTCACA \\
\hline HM\#345 & nosP_Bbsi_F & CGGAAGACCGCGATTCCTTCAGTATCTCCAAATCGCCCCGGAC \\
\hline HM\#368 & attP220_Fwd & TCATCAATGTATCACTAGTACTGACGGACACACC \\
\hline HM\#369 & attP220_Rev & CTGGCTGGGGAATCTGTACTAGTCGCGCTCG \\
\hline HM\#469 & Spel_gypsy_F & GCTTACTAGTGATGGTCTCAAGCTTGTCAGATCGGC \\
\hline HM\#470 & Apal_SV40_R & TTAGGGCCCCGCCTTAAGATACATTGATGAGTTTGG \\
\hline HM\#560 & FH_Cas9_HidllI_F & CATCAAGCTTACAAGTTCATCAAGCCCATCCTGG \\
\hline HM\#561 & FH_Cas9_Xhol_R & CATGCTCGAGATAGGTTTTCAGCCGTTCCTCGATC \\
\hline HM\#584 & HM_EcoRI_TRE_F2 & TACGAATTCGGCGCGCCTAGGCCGGCCGAATTTC \\
\hline HM\#706 & HM_b2t_3UTR_F & CGAGGATCCTAGGATTAACTTCCCACTCAAGATCACACATG \\
\hline HM\#707 & HM_b2t_3UTR_R & GCCAAGCTTGTCTGCTTATAAATCAACATTTATTCGTAACCC \\
\hline mfs\#370 & AflII-5pBac_F & AACTTAAGTTAACCCTAGAAAGATAGTCTGC \\
\hline MK153 & PhiC31_Bsal_F & ATGGTCTCACATGGACACGTACGCGGGTGCTTACGAC \\
\hline T7 & T7 & TAATACGACTCACTATAGGG \\
\hline CH6R & CH_3'PIClal_2 & CCATCGATGGAATGAACAGGACCTAACGC \\
\hline
\end{tabular}


Additional File 2

\section{Supplementary Table 2: List of primers used}

\begin{tabular}{|c|c|c|}
\hline Code & Name & Sequence 5'- 3' \\
\hline HM\#16 & sryaR4 & TTGTGTGTCATGGATGTTCAATCTAATC \\
\hline HM\#17 & Srya5UTR_F1 & GTACTTAGTTGAAAAGTTCAGCTTTACCCG \\
\hline HM\#34 & Ds_srya_GSP3 & GGCATCCAGGCTAATGGTCCGCTCCAAGTG \\
\hline HM\#35 & B2t_Ncol_F1 & GCAACCATGGGATGCCAAGAGAGATGAGCAGG \\
\hline HM\#36 & B2t_Xbal_R3 & CGATTCTAGACATCTTAACCGACTGTCAAGGATC \\
\hline HM\#42 & srya_GSP_F & GCCTCTCTGGCTCCGATTCCCCCTAATG \\
\hline HM\#73 & hspEcoRIF2 & GCGAATTCTCCAGAAACTCAAACAGAAACC \\
\hline HM\#74 & hspEcoRIR & GCGAATTCTTGTGTGTTTGTGTTTGTGGATGCAG \\
\hline HM\#76 & nos_GSPR & GAGTCCTCCTCTTGCGTGGAATGCCG \\
\hline HM\#77 & nos_GSPF & GTACTGTCCCAAGAAGCCGATTATCAC \\
\hline HM\#94 & nos3UTRXbalF & GGTCTAGAGAACACATCCGGCAGGAGC \\
\hline HM\#95 & nos3UTRAfIIIR & ATACTTAAGACTGAGCTCCAAGCAGTGGTATCAACGCAGAG \\
\hline HM\#101 & Spel-atBF & CTAGTGTGAGGTGGAGTACGCGCCCGGGGAGCCCAAGGGCACGCCCTGGCACCCGCAC \\
\hline HM\#102 & Bbsl-Bbsl & CGGTCTTCGCGAAGAC \\
\hline HM\#113 & Bbsl_nosR & GCGAAGACCCATATGGCGAAAGTCCGGCTCGAAAGTTACC \\
\hline HM\#117 & HM_Pub_R & CATTGGAATCTCTGTCGCTGCGTTCCG \\
\hline HM\#123 & phi_nls_R & СтAGACCTTCCGCTTCTTCTTTGGGGCCGCCGCTACGTCTTCCGTGCCGTCCTG \\
\hline HM\#124 & SV40_Spel_R & CACACTAGTGATACATTGATGAGTTTGGACAAACCACAAC \\
\hline HM\#131 & PLF2 & GTCAAAATGACGCATGATTATCTTTTACG \\
\hline HM\#179 & DsRed_End & CGAGGGCCGCCACCACCTGTTCCTG \\
\hline HM\#203 & Notl_phiC31_R & TCGCGGCCGCCTAGACCTTCCGCTTCTTCTTTGG \\
\hline HM\#337 & Spel_attB_R & СTAGGTGCGGGTGCCAGGGCGTGCCCTTGGGCTCCCCGGGCGCGTACTCCACCTCACA \\
\hline HM\#345 & nosP_Bbsi_F & CGGAAGACCGCGATTCCTTCAGTATCTCCAAATCGCCCCGGAC \\
\hline HM\#368 & attP220_Fwd & TCATCAATGTATCACTAGTACTGACGGACACACC \\
\hline HM\#369 & attP220_Rev & СTGGCTGGGGAATCTGTACTAGTCGCGCTCG \\
\hline HМ\#469 & Spel_gypsy_F & GCTTACTAGTGATGGTCTCAAGCTTGTCAGATCGGC \\
\hline HM\#470 & Apal_SV40_R & TTAGGGCCCCGCCTTAAGATACATTGATGAGTTTGG \\
\hline HM\#560 & FH_Cas9_HidlII_F & CATCAAGCTTACAAGTTCATCAAGCCCATCCTGG \\
\hline HM\#561 & FH_Cas9_Xhol_R & CATGCTCGAGATAGGTTTTCAGCCGTTCCTCGATC \\
\hline HM\#584 & HM_EcoRI_TRE_F2 & TACGAATTCGGCGCGCCTAGGCCGGCCGAATTTC \\
\hline HM\#706 & HM_b2t_3UTR_F & CGAGGATCCTAGGATTAACTTCCCACTCAAGATCACACATG \\
\hline HM\#707 & HM_b2t_3UTR_R & GCCAAGCTTGTCTGCTTATAAATCAACATTTATTCGTAACCC \\
\hline mfs\#370 & Aflll-5pBac_F & AACTTAAGTTAACCCTAGAAAGATAGTCTGC \\
\hline MK153 & PhiC31_Bsal_F & ATGGTCTCACATGGACACGTACGCGGGTGCTTACGAC \\
\hline T7 & $\mathrm{T7}$ & TAATACGACTCACTATAGGG \\
\hline $\mathrm{CH} 6 \mathrm{R}$ & CH_3'PIClal_2 & CCATCGATGGAATGAACAGGACCTAACGC \\
\hline
\end{tabular}




\subsection{Reproductive Sterility System for Drosophila suzukii control based on knock- out or knock-down of specific male fertility genes}

This chapter describes the first steps towards the development of a reproductive sterility system for the control of the invasive fruit pest $D$. suzukii. The system is based on the conditional knockout or knockdown of the paternal effect lethal (PEL) gene sneaky (snky), which is important for sperm plasma membrane breakdown (PMBD) after fertilization. Failure of this process leads to embryonic lethality. We want to utilize the conditional tet-off system and CRISPR/Cas9 to knockout snky during spermatogenesis and thereby introduce reproductive sterility. We also describe an approach for conditional expression of short hairpin RNAs to knockdown snky during spermatogenesis as a second mechanistically independent method to induce reproductive sterility. The described strategy to target PEL genes, which do not affect male fitness, sperm production or transfer, but exert the effect only after sperm use and entrance into the egg during zygote formation, present a novel approach for improvement of the SIT. If we succeed, we generate a new foundation for the control of $D$. suzukii using the genetic pest strategy SIT.

Hassan M. M. Ahmed \& Ernst A. Wimmer ${ }^{1}$

Authors contribution to the practical work:

Hassan M. M. Ahmed: All experiments.

Status: $\quad$ Work in progress. 


\title{
Reproductive Sterility System for Drosophila suzukii control based on knock-out or knock-down of specific male fertility genes
}

\author{
Hassan M. M. Ahmed ${ }^{1,2}$, \& Ernst A. Wimmer ${ }^{1^{*}}$ \\ ${ }^{1}$ Department of Developmental Biology, Johann-Friedrich-Blumenbach-Institute of Zoology and Anthropology, \\ Göttingen Centre for Molecular Biosciences, University of Göttingen, 37077 Göttingen, Germany. ${ }^{2}$ Department of Crop \\ Protection, Faculty of Agriculture-University of Khartoum P.O. Box 32, Postal Code 13314 Khartoum North, Khartoum, \\ Sudan \\ *Corresponding Author: E. A. Wimmer. Tel +49 5513922888. \\ E-Mail address: ewimmer@gwdg.de (E.A. Wimmer)
}

\section{Background}

The sterile insect technique (SIT) is considered an important component of area wide integrated pest management (AW-IPM) (1). The system is based on mass rearing and inundative releases of sterile males of the target species to compete with the wild type males for the wild type females leading to infertile mating and hence reduction of the population (2). The sterility is so far introduced by exposing the pupae to ionizing radiation which leads to chromosome breaks resulting in different chromosomal aberrations causing aneuploidy in the sired embryos, which is the basis of the reproductive sterility $(2,3)$. However, irradiation has negative impact on the fitness of the sterile males to be released. To overcome this, scientists exploited the tools of molecular biology to engineer transgenic conditional embryonic lethality systems that preclude the need for ionizing radiation. The system is controlled by the tet-off binary system and an enhancer/promoter of an early embryonic stage to express a proapoptotic gene $(4,5)$. Another approach that was engineered to induce reproductive sterility is the release of insects carrying a dominant lethal RIDL (6). This system also relies on the use of the tet-off system but in this case the effector is a heterologous transcription factor, the tetracycline-controlled transactivator (tTA), which is placed downstream of the tet operator and a weak basal promoter (6). In the absence of tetracycline, it leads to establishment of positive feedback loop of tTA which results in cytotoxicity.

The emergence of the bacterial adaptive immune system CRISPR/Cas, an acronym of Clustered Regularly Interspaced Short Palindromic Repeat and the Associated protein, as tool

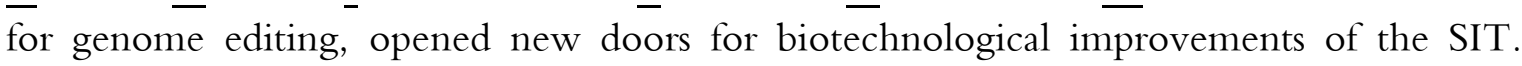
Eckermann et al. (7) proposed the use of the system to develop multifactorial reproductive sterility system by expression of Cas 9 during spermatogenesis and targeting it to repetitive elements in the genome and thereby induce many chromosomal breaks, which eventually leads to chromosomal aberrations and reproductive sterility. Beside the system proposed earlier (Eckermann et al., (7), the CRISPR/Cas system can also be used in various other ways to introduce reproductive sterility. However, when targeting male fertility genes, it needs to 
be emphasized that the sterile males should still produce competitive sperm keeping the ability of sperm transfer to females. Furthermore, the transferred sperm should be able to compete with wild type sperm, since in many of the target pests the females mate more than once (polyandry). Very few genes causing paternal effect embryonic lethality (PEEL) when mutated were identified, which don't interfere with spermatogenesis, but the downstream steps involved in fertilization or gamete fusion. An example of such a mutation that affect spermegg recognition is casanova ( $\operatorname{csn}$ ) (8), which causes the sperm failing to enter the egg. The failure is attributed to a lack of $\beta-\mathrm{N}$-acetylglucosaminidase on the plasma membrane covering the acrosome. Another gene causing PEEL is sneaky (snky), which is expressed explicitly in the testes of Drosophila during spermatogenesis and codes for an acrosomal protein involved in sperm plasma membrane breakdown (PMBD) during fertilization $(9,10)$. Knockout of this gene does not affect male fitness or sperm competence (9). Sperm is normally produced, transferred and utilized to fertilize the egg, however, the sperm fails to form a functional male pronucleus. The Drosophila gene misfire ( $m f f$ is expressed in the ovaries and testes and encodes a membrane protein, which is also required for PMBD and activation of the sperm during fertilization and a null mutant is therefore male sterile (11)

Originally identified in plants (12-14) and the nematode Caenorhabditis elegans (15) RNA interference pathway has been found in all studied organisms so far. It is a mechanism of post transcription regulation of gene expression as well as a defence mechanism against parasitic genetic elements such as viruses and transposable elements (16). The RNAi pathway is activated by dsRNA molecule which is processed to short 21-27 dsRNAs fragments by an enzyme called Dicer (17). The small duplexes are then unwound into ssRNA molecules, the targeting strand is then incorporated into the RNA-silencing complex (RISC) and guided the complex by complementary based pairing to target and degrade mRNA. (17)

Since its discovery, the system has been intensively used in reverse genetics to study the function of gene by knocking down the gene and studying the resulting phenotype (18). This has been exploited to perform systematic large-scale screen in D. melanogaster (19-21), and Tribolium castaneum (22-24). The RNAi pathway can be triggered by direct injection of the dsRNA into the embryos, or also in other stages in case of systemic effect (25). It can also be triggered by expression of dsRNA or short hairpin RNA from a transgene using either inducible promoters or binary expression system such as Gal4/UAS(26). The RNAi beside its broad applications in functional genetic studies and its potentials in gene therapy (27), has also been explored for its applicability to engineer transgenic strains suitable for the SIT. Knockdown of the sex determination gene transformer or transformer 2 in the tephritid flies and possibly in some other insect leads to female to male conversion $(28,29)$.

Development of a reproductive sterility system by targeting paternal effect genes for knockout or down necessitates that the effector molecule (e.g. Cas9 or a dsRNA) be restricted to 
spermatogenesis, which makes identification of suitable regulatory elements a prerequisite. The Drosophila $\beta$ Tub85D $(\beta 2 t)$ gene is known to be spermatogenesis-specific and is active in all the stages of sperm production starting at the third larval instar (30). The enhancer/ promoter of this gene has been used to drive strong expression of reporter genes such as lac $Z$ and fluorescent proteins such as EGFP that facilitate basic research in male reproductive biology (30). The $E / P$ of the $\beta 2 t$ gene has also been used to generate sperm-marking strains to help monitoring released males in SIT programs (31-34). Furthermore, it has been used to drive the expression of a homing endonuclease gene (HEG), the I-PpoI, in the malaria mosquito in a biotechnological vector control approach, where the HEG targets conserved sequences within the ribosomal DNA in the X chromosome of Anopheles gambiae and shred it leading to Y chromosome biased sperm and thus sex ratio distortion (35). The Drosophila genes don juan ( $d j$ ) and don juan like (dji) are testes-specifically expressed with a translation repression element in their 5' UTR that delay translation of the mRNA to post-meiotic stage of spermatogenesis (36). Since those elements are well characterized, their deletion can allow the use of the respective promoters also for early spermatogenesis expression.

Here, we have chosen to target the PEEL gene snky which is involved in sperm plasma membrane breakdown during fertilization (10). Homozygous null mutants of this gene display complete male sterility. We proposed the use of CRISPR/Cas9 or RNA interference to conditionally knock-out or knock-down, respectively, this gene individually or in combination for production of reproductively sterile males to establish the sterile insect technique for the control of the invasive fruit pest $D$. suzukii with first proof-of-concept in D. melanogaster.

\section{Results}

\section{Identification and validation of suitable CRISPR targets}

Before identifying suitable CRISPR target sites in D. melanogaster PEEL gene snky, Fig. 1A, the gene was PCR-amplified and sequenced to avoid variations that may affect target recognition or lowers the efficiency of targeting. Using the online target finder tool developed by the university of Wisconsin (https://flycrispr.org/protocols/), we identified and designed four $g R N A$ s with zero off target sites in D. melanogaster genome (37), Dm_snky_g3, Dm_snky_g13,Dm_snky_g38, and Dm_snky_g41 (Fig. 1A and 1B). The four gRNAs were subsequently cloned into vector HMMA332 to express the $g R N A s$ from Dm U6:3 promoter, injected along with Cas9 plasmid HMMA056 into D. melanogaster embryos and their efficiency was evaluated by T7 EndoI assay. Based on the results, the three guides, Dm_snky_g13,Dm_snky_g38, and Dm_snky_g41, are chosen to develop the system (Fig. $1 \mathrm{C})$. 


\section{Spermatogenesis-specific gRNAs-driver constructs and transgenic lines.}

To achieve conditional knockout or knockdown of snky by CRISPR/Cas9 or by expression of short hairpin RNAs $(\operatorname{sh} R N A)$ respectively, we used the tet-off binary expression system and the enhancer/ promoter (E/P) of $D$. melanogaster spermatogenesis specific genes $\beta 2 t$ and Don juan (dj). We fused the E/P of $D m-\beta 2 t$ or $D m-d j$ (without the $d j$ TSE to allow premeiotic translation of $\mathrm{tTA}$ ) upstream of the heterologous transcription factor tetracycline transactivator $(t T A)$. The spermatogenesis-specific driver construct HMMA389 for D. suzukii is described elsewhere (chapter 3.4).

To be able to conditionally and simultaneously express two $g R N A s$, we made use of the tetoff binary expression system and the $t R N A$ processing system for $g R N A$ multiplexing (3840). and generated vectors HMMA324 and HMMA325 by fusion of a synthetic multiplexing cassette consisting of two $g R N A$ scaffolds interspaced and flanked by three $t R N A$ genes to the TREp and TREhs43 promoters respectively. This will allow the release of two mature $g R N A s$ after processing of the $t R N A$ genes by the highly conserved ribonucleases $\mathrm{P}$ and $\mathrm{Z}$ (41) (Fig. 2B and 2C). The vector HMMA324 was used to eventually generate the four driver constructs HMMA371, HMMA372, HMMA373, and HMMA374 that in addition to mediate $t T A$ expression also express $g R N A$ s targeting $D m-s n k y$ (Fig. 2A and 2B). The construct HMMA372 that expresses $t T A$ under $D m-\beta 2 t E / P$ and $g R N A s D m \_s n k y \_g 13$, and $D m \_s n k y \_g 41$ was used to generate spermatogenesis-specific $g R N A$-driver lines. Interestingly, the majority of the $\mathrm{F}_{1}$ males were sterile. These driver lines will be crossed to Cas 9 responder lines (see below) to transactive expression of Cas 9 during spermatogenesis and knockout the Dm-snky gene

In addition, we generated the spermatogenesis-specific driver constructs HMMA425 ( $D s-\beta 2 t$ $E / P)$, HMMA426(Dm- $\beta 2 t \mathrm{E} / \mathrm{P})$ and HMMA427 (Dm-dj E/P) by cloning the coding sequence of the turbo green fluorescent protein gene $(t G F P)$ in frame with $t T A$ and separated by the picornavirus $P 2 A$ peptide sequence. The $t G F P$ gene serves as a reporter for the tissue specific expression of the $t T A$ and should later facilitate analysis of the functionality of the system by following the ability of the males to transfer viable sperm to the females, the entrance of the sperm in the egg and the failure of PMB during fertilization.

Moreover, we generated vectors HMMA349 and HMMA410 that allow constitutive expression of three $g R N A s$ simultaneously based on the $t R N A$ processing system and the promoter of the RNA pol III small nuclear genes $U 6: 3$ and $U 6 c$ from D. melanogaster and $D$. suzukii, respectively. We then engineered spermatogenesis specific gRNA-driver constructs HMMA433 and HMMA434 that express $t T A:: 2 A:: t G F P$ from $E / P$ of $D m-\beta 2 t$ or $D m-d j$ genes, respectively, plus $g R N A s D m \_s n k y \_g 13, D m \_s n k y \_g 38$, and Dm_snky_g41 from $U 6: 3$ promoter (Fig. 4A). 


\section{CRISPR/Cas9 responder constructs and transgenic lines}

The second part of the proposed CRISPR/Cas9 reproductive sterility system consist of Cas 9 coding sequence fused to the $t T A$ responsive element (TRE) and a basal promoter (Fig. 3B). We generated the responder constructs HMMA338 by fusion of the insect codon optimized Cas 9 coding sequence (with $\mathrm{N}$ and $\mathrm{C}$-termini nuclear localization signal(nls) and $\mathrm{N}$-terminus 3 XFlag tag) downstream of the TRE and the P-element basal promoter. This should allow pre-meiotic translation of Cas $9 \mathrm{mRNA}$. The construct was used to generate several responder lines.

\section{Cross of gRNA-driver lines to Cas9 responder lines}

To generate a reproductive sterility strain (RSS), all the components of the reproductive sterility system must be combined in one strain, to do so we crossed the gRNA-driver lines 372_F26F1 and 372_M14M1 separately to the responder lines 338_F6M1, 338_F13F1 and 338_M10M2 (Fig. 3A and 3B). Supplementing the flies' food with tetracycline should supress the expression of the effector molecules Cas 9 and the $g R N A s$ by preventing the heterologous transcription factor tTA from binding to the TRE promoter (Fig. 3A). In the absence of tetracycline, the tTA is free to bind to $T R E$ and thereby activates the expression of Cas 9 and the $g R N A s$ (Fig. 3B). The $g R N A s-t R N A s$ transcript should then be processed by the ribonucleases $\mathrm{P}$ and $\mathrm{Z}$ and free the two $g R N A s$ Dm_snky_g13 and Dm_snky_g41 to form the Cas9-gRNA Ribonucleoprotein complex (RNP) which should then target Dm-snky. (Fig. 4C)

Activation of the system in double homozygous males for the gRNAs-driver and responder transgene after withdrawal of the tetracycline from the food leads to expression of Cas 9 and the $g R N A s$ starting at the $3^{\text {rd }}$ larval instar in the gonads, which then should target and induce double strand breaks to knockout the male fertility gene snky. This should result in the production of competent males that produce viable sperm, which effectively enters the egg but embryonic development will not occur due to failure of the formation of a functional male pronucleus.

\section{Testes specific expression of Cas 9}

A preliminary reverse transcriptase PCR (RT-PCR) experiment was performed to investigate, whether Cas9 is expressed in the testes of the RSSs. A pair of primers was used to PCR amplify a 460bp fragment of Cas9, and another pair was used for a positive control amplifying the 3'UTR of $D m-\beta 2 t$. As negative controls, we performed RT-PCR using total RNA from the respective responder lines 338_F6M1, 338_F13F1, and 338_M10M2 not crossed to driver lines as well as no RT PCR for all the different strains. Cas 9 transcript was detected in all three RSSs, interestingly it has also been detected in the negative control 
responders not crossed to driver lines but not in the no RT PCR (Fig. 3C). This might be due to leakiness in the expression of Cas9 from the basal $P$-element promoter.

\section{Over expression of $t T A$ may cause male sterility}

Out of 14 independent $F_{1}$ transgenic gRNA-driver lines generated, 5 were females and 9 were males of which 7 were sterile males that didn't sire offspring. It is well known that overexpression of $t T A$ can cause cytotoxicity (6). Due to the way the driver construct HMMA372 was generated, with TREp upstream of the $\beta 2 t$ promoter, a positive selfsustaining loop may have formed that leads to over-expression of $t T A$ This might well be a reason of cell toxicity resulting in male sterility. Besides, the 3'UTR of the $D m-\beta 2 t$ gene fused downstream of $t T A$ is also known to lead to overexpression (42) (Fig. $2 \mathrm{~B}$ and $3 \mathrm{~A}$ ).

\section{Conditional Knockdown of sneaky}

Another approach to generate RSSs is by knocking down the expression of the male fertility genes such as snky by expression of $s h R N A$ or double strand RNA ( $d s R N A$ ) during spermatogenesis which will then induce RNA interference depletion of snkymRNA. To this end, we utilized the tet-off binary expression system and the $t R N A$ processing system to engineer responder construct HMMA309 and HMMA310 that allow expression of two $s h R N A s$ or $d s R N A s$. We designed two $21 \mathrm{bp}$ long $s h R N A s$ (shRNA_13 and shRNA_41) with a $9 \mathrm{bp}$ loop driven from $D$. melanogaster mir14 and generated the responder construct HMMA445 (Fig. 4A). This construct is being used to generate transgenic responder lines. This system, if proved to be effective in inducing male sterility, can be used as stand-alone or in combination with CRISPR/Cas9 reproductive sterility system to generate a double hit reproductive sterility system to avoid resistance development (Fig 4.B).

\section{Identification of $D$. suzukii orthologue of the gene sneaky}

To be able to eventually transfer the system to the invasive fruit pest $D$. suzukii, it is necessary to identify suitable paternal effect genes. Searching in D. suzukii database (http://spottedwingflybase.org/) with D. melanogastersequence of the gene snky as query, we were able to identify homologous sequences that shares $76 \%$ and $89 \%$ similarity at the nucleotide and amino acid levels respectively to D. melanogaster counterparts. Part of the gene including exon I was PCR amplified from genomic DNA and sequenced.

\section{Discussion}

In this study we describe the first steps towards the development of a new reproductive sterility system for the fight of the invasive fruit pest D. suzukii. The idea is to use the CRISPR/Cas9 system or the RNA-interference approach to target and knockout (knock-down) the paternal effect lethal gene snky during spermatogenesis. This gene encodes an acrosomal protein which is expressed exclusively during spermatogenesis but is not necessary for the successful completion of this biological process (10). Homozygous mutant males are sterile in the sense 
that they don't sire progeny despite the fact that they produce morphologically functional sperm that can be efficiently transferred to females during copulation and can enter the egg but does not form a functional male pro-nucleus due to failure of PMBD (10). These characteristics of snky mutants are very important for developing the reproductive sterility system.

The CRISPR/Cas9 system was used previously to knock out $D m-\beta 2 t$ gene which results in male sterility (43) however, those males don't produce functional sperm. In such a case, the females will search for other males, which suggest that $D m-\beta 2 t$ is not a suitable target to induce sterility for application in the SIT programs. Our proposed system is controlled both by the tissue specific promoter of $D m-\beta 2 t$ gene and the tet-off binary expression system to allow conditional activation when desired. We used the construct HMMA372 consisting of the $E / P$ of $D m-\beta 2 t$ gene to drive the expression of $t T A$ and a gRNA cassette $(\mathrm{g} 13, \mathrm{~g} 41)$ under the control of TRE to generate transgenic gRNA-driver lines (Fig. 2A and 2B). We observed male sterility in 7 out of 9 independent $F_{1}$ transgenic males and managed to establish 7 transgenic lines, only two of them were $F_{1}$ fertile males and the other 5 were established from $\mathrm{F}_{1}$ females. This sterility might be attributed to either of two factors or a combination thereof. First, the use of the TRE upstream of the $D m-b 2 t$ promoter can lead to enhancement of expression of $t T A$ by establishment of a positive feed-back loop (6) (Fig. 2B). Accumulation of tTA can be toxic to the cells but the mechanism of this toxicity is so far unresolved. In fact, such a construct has been made intentionally to develop a reproductive sterility system known as RIDL, an acronym of release of insects carrying a dominant lethal, which results in cytotoxicity and death (6). Secondly, the 3'UTR of $D m-b 2 t$ is known to control the level of expression (42) and gives high expression but with slight delay in translation (44). In our construct HMMA372, we have fused the 3'UTR from Dm-b2t gene to the transcription factor encoding gene $t T A$. So, the simplest explanation is that the two factors might be involved in the toxicity and can explain the observed male sterility. This can be overcome by either using an $R N A$ polIII-based strategy for expression of $g R N A s$ (Fig. 2C) or place the $g R N A s$ cassette along with the $\operatorname{Cas} 9$ responder construct and not with the driver construct. Whether or not the 3'UTR alone can lead to overexpression of $t T A$ to levels that are toxic needs to be determined empirically.

To investigate the functionality of the gRNA-driver lines we crossed two lines to three different Cas 9 responder lines and performed a preliminary RT-PCR. We observed Cas9 transcript in all 6 crosses plus the negative controls (uncrossed Cas 9 responder lines) (Fig. 3C). It is obvious that this is not genomic DNA contamination due to the fact that the respective negative control (no RT PCR) didn't show amplification for all the crosses and the Cas9 responder negative controls (Fig. 3C). Some level of expression from the basal promoter is expected and might not interfere with the functionality of the strain but that has to be determined. This basal expression can be minimized by flanking the transgene with insulators 
such as the chicken hypersensitive site 4 (HS4) from the chicken $\beta$-globin gene to avoid activation by surrounding enhancer elements in the site of integration or in the vector itself. Especially as the construct has the E/P of the D. melanogaster polyubiquitin gene.

The utilization of the RNA interference pathway to knockdown snky provides another approach for generation of the reproductive sterility system. In this respect we developed responder constructs based on the tet-off and the $t R N A$ processing systems to conditionally drive the expression of two $s h R N A s$ or $d s R N A$ (Fig 4B). Two $21 \mathrm{bp} \operatorname{sh} R N A s$ targeting $D$. melanogaster snky were generated with a $9 \mathrm{bp}$ loop derived from $D$. melanogaster mir14, which proved suitable in Schneider cells (45). After testing this system, it can be combined with the CRISPR/Cas9 system described above to have two mechanisms for induction of sterility (Fig. 4B). Each system acts as a surveillance mechanism for the other to eliminate any escapers and ensure 100\% reproductive sterility (Fig. 4C and 4E).

It has been suggested that the $\beta 2 t$ gene like many other genes involved in spermatogenesis probably has a translation delay element within the 5' UTR (44). Deletion of this element from the $P / E$ of the medfly Ceratitis capitata permitted early transcription and translation of genes fused to it (46). Early expression of tTA ensures early expression of the downstream effector molecule (Cas9 and/or shRNA) before shut-down of transcription in spermatogenesis. Removal of the translation repression element should also be possible in $D$. melanogaster, since its regulatory elements are well characterized. Moreover, as an alternative to $D m-b 2 t E / P$, we can use the $P / E$ of the germline specific genes nanos or $R c d-1 r$ to drive the expression of $t T A$ particularly since snky is not required and not expressed in the females and is not required for males. Another promoter that could be used instead is the one from the bag of marbels gene (bam), which is expressed in early testes primordial germ cells early enough for the GAL4 system to be used in spermatogenesis studies in D. melanogaster (47)

The two approaches described for the generation of a reproductive sterility system in $D$. melanogaster, if proven successful can easily be developed for the fruit pest $D$. suzukii, since we have already identified the $D s-s n k y$ gene, and sequenced it. This system along with the sperm-marking already developed (34) would be a giant leap forward towards the establishment of the sterile insect technique against $D$. suzukii. 


\section{References}

1. Klassen W. Area-Wide Integrated Pest Management and the Sterile Insect Technique. In: Dyck VA, Hendrichs J, Robinson AS, editors. Sterile Insect Technique [Internet]. Berlin/Heidelberg: Springer-Verlag; 2005 [cited 2019 Nov 17]. p. 39-68. Available from: http://link.springer.com/10.1007/1-4020-4051-2_2

2. Knipling EF. Possibilities of Insect Control or Eradication Through the Use of Sexually Sterile Males1. Journal of Economic Entomology. 1955 Aug 1;48(4):459-62.

3. Bakri A, Mehta K, Lance DR. Sterilizing Insects with Ionizing Radiation. In: Dyck VA, Hendrichs J, Robinson AS, editors. Sterile Insect Technique: Principles and Practice in AreaWide Integrated Pest Management [Internet]. Dordrecht: Springer Netherlands; 2005 [cited 2019 Nov 17]. p. 233-68. Available from: https://doi.org/10.1007/1-4020-4051-2_9

4. Horn C, Wimmer EA. A transgene-based, embryo-specific lethality system for insect pest management. Nat Biotechnol. 2003 Jan;21(1):64-70.

5. Schetelig MF, Caceres C, Zacharopoulou A, Franz G, Wimmer EA. Conditional embryonic lethality to improve the sterile insect technique in Ceratitis capitata(Diptera: Tephritidae). BMC Biol. 2009 Dec;7(1):4.

6. Thomas DD, Donnelly CA, Wood RJ, Alphey LS. Insect Population Control Using a Dominant, Repressible, Lethal Genetic System. Science. 2000 Mar 31;287(5462):2474-6.

7. Eckermann KN, Dippel S, KaramiNejadRanjbar M, Ahmed HM, Curril IM, Wimmer EA. Perspective on the combined use of an independent transgenic sexing and a multifactorial reproductive sterility system to avoid resistance development against transgenic Sterile Insect Technique approaches. BMC Genet. 2014;15(Suppl 2):S17.

8. Perotti M-E, Cattaneo F, Pasini ME, Vernì F, Hackstein JHP. Male sterile mutant casanova gives clues to mechanisms of sperm-egg interactions in Drosophila melanogaster. Molecular Reproduction and Development. 2001;60(2):248-59.

9. Fitch KR, Wakimoto BT. The Paternal Effect Genems(3)sneakyIs Required for Sperm Activation and the Initiation of Embryogenesis inDrosophila melanogaster. Developmental Biology. 1998 May 15;197(2):270-82.

10. Wilson KL, Fitch KR, Bafus BT, Wakimoto BT. Sperm plasma membrane breakdown during Drosophila fertilization requires Sneaky, an acrosomal membrane protein. Development. 2006 Dec 15;133(24):4871-9.

11. Ohsako T, Hirai K, Yamamoto M-T. The Drosophila misfire gene has an essential role in sperm activation during fertilization. Genes Genet Syst. 2003 Jun;78(3):253-66. 
12. Matzke MA, Matzke AJM. How and Why Do Plants lnactivate Homologous (Trans)genes?'. Plant physiol. 1995;107:7.

13. Ratcliff F, Harrison BD, Baulcombe DC. A Similarity Between Viral Defense and Gene Silencing in Plants. Science. 1997 Jun 6;276(5318):1558-60.

14. Hamilton AJ, Baulcombe DC. A Species of Small Antisense RNA in Posttranscriptional Gene Silencing in Plants. Science. 1999 Oct 29;286(5441):950-2.

15. Grishok A, Pasquinelli AE, Conte D, Li N, Parrish S, Ha I, et al. Genes and Mechanisms Related to RNA Interference Regulate Expression of the Small Temporal RNAs that Control C. elegans Developmental Timing. Cell. 2001 Jul 13;106(1):23-34.

16. Elbashir SM, Lendeckel W, Tuschl T. RNA interference is mediated by 21 - and 22-nucleotide RNAs. Genes Dev. 2001 Jan 15;15(2):188-200.

17. Wilson RC, Doudna JA. Molecular Mechanisms of RNA Interference. Annual Review of Biophysics. 2013;42(1):217-39.

18. Tenea GN, Burlibasa L. RNAi Towards Functional Genomics Studies. Functional Genomics [Internet]. 2012 Sep 12 [cited 2019 Nov 17]; Available from: https://www.intechopen.com/books/functional-genomics/rnai-towards-functional-genomicsstudies

19. D’Ambrosio MV, Vale RD. A whole genome RNAi screen of Drosophila S2 cell spreading performed using automated computational image analysis. J Cell Biol. 2010 Nov 1;191(3):471-8.

20. Bakal C. Drosophila RNAi screening in a postgenomic world. Briefings in Functional Genomics. 2011 Jul 1;10(4):197-205.

21. Heigwer F, Port F, Boutros M. RNA Interference (RNAi) Screening in Drosophila. Genetics. 2018 Mar;208(3):853-74.

22. Brown SJ, Shippy T, Miller S, Bolognesi R, Beeman RW, Lorenzen MD, et al. The red flour beetle, Tribolium castaneum (Coleoptera): a model for studies of development and pest biology. Cold Spring Harbor protocols. 2009;2009(8):pdb.emo126.

23. Schultheis D, Weißkopf M, Schaub C, Ansari S, Dao VA, Grossmann D, et al. A Large Scale Systemic RNAi Screen in the Red Flour Beetle Tribolium castaneum Identifies Novel Genes Involved in Insect Muscle Development. G3. 2019 Feb 7;g3.200995.2018.

24. Schmitt-Engel C, Schultheis D, Schwirz J, Ströhlein N, Troelenberg N, Majumdar U, et al. The iBeetle large-scale RNAi screen reveals gene functions for insect development and physiology. Nat Commun. 2015 Nov;6(1):7822. 
25. Miller SC, Miyata K, Brown SJ, Tomoyasu Y. Dissecting Systemic RNA Interference in the Red Flour Beetle Tribolium castaneum: Parameters Affecting the Efficiency of RNAi. PLOS ONE. 2012 Oct 25;7(10):e47431.

26. Matsushima Y, Adán C, Garesse R, Kaguni LS. Functional Analysis by Inducible RNA Interference in Drosophila melanogaster. In: Leister D, Herrmann JM, editors. Mitochondria [Internet]. Totowa, NJ: Humana Press; 2007 [cited 2019 Nov 3]. p. 207-17. Available from: http://link.springer.com/10.1007/978-1-59745-365-3_15

27. Caplen NJ. RNAi as a gene therapy approach. Expert Opinion on Biological Therapy. 2003 Jul 1;3(4):575-86.

28. Schetelig MF, Milano A, Saccone G, Handler AM. Male only progeny in Anastrepha suspensa by RNAi-induced sex reversion of chromosomal females. Insect Biochemistry and Molecular Biology. 2012 Jan 1;42(1):51-7.

29. Liu G, Wu Q, Li J, Zhang G, Wan F. RNAi-Mediated Knock-Down of transformer and transformer 2 to Generate Male-Only Progeny in the Oriental Fruit Fly, Bactrocera dorsalis (Hendel). PLOS ONE. 2015 Jun 9;10(6):e0128892.

30. Michiels F, Gasch A, Kaltschmidt B, Renkawitz-Pohl R. A 14 bp promoter element directs the testis specificity of the Drosophila 32 tubulin gene. The EMBO Journal. 1989;8(5):1559-65.

31. Catteruccia F, Benton JP, Crisanti A. An Anopheles transgenic sexing strain for vector control. Nat Biotechnol. 2005 Nov;23(11):1414-7.

32. Scolari F, Schetelig MF, Bertin S, Malacrida AR, Gasperi G, Wimmer EA. Fluorescent sperm marking to improve the fight against the pest insect Ceratitis capitata (Wiedemann; Diptera: Tephritidae). New Biotechnology. 2008 Jun;25(1):76-84.

33. Zimowska GJ, Nirmala X, Handler AM. The $\beta 2$-tubulin gene from three tephritid fruit fly species and use of its promoter for sperm marking. Insect Biochemistry and Molecular Biology. 2009 Aug;39(8):508-15.

34. Ahmed HMM, Hildebrand L, Wimmer EA. Improvement and Use of CRISPR/Cas9 to Engineer a Sperm-marking Strain for the Invasive Fruit Pest Drosophila suzukii. 2019 Aug 14 [cited 2019 Nov 2]; Available from: https://www.researchsquare.com/article/804bbff3-0228-44d5-8e09_ $3 \mathrm{acb} 0 \mathrm{~d} 8 \mathrm{e} 408 \mathrm{e} / \mathrm{v} 1$

35. Galizi R, Doyle LA, Menichelli M, Bernardini F, Deredec A, Burt A, et al. A synthetic sex ratio distortion system for the control of the human malaria mosquito. Nat Commun. 2014 Sep;5(1):3977.

36. Hempel LU, Rathke C, Raja SJ, Renkawitz-Pohl R. In Drosophila, don juan and don juan like encode proteins of the spermatid nucleus and the flagellum and both are regulated at the 
transcriptional level by the TAFII80 cannonball while translational repression is achieved by distinct elements. Developmental Dynamics. 2006;235(4):1053-64.

37. Hoskins RA, Carlson JW, Wan KH, Park S, Mendez I, Galle SE, et al. The Release 6 reference sequence of the Drosophila melanogaster genome. Genome Res. 2015 Mar 1;25(3):445-58.

38. Port F, Bullock SL. Augmenting CRISPR applications in Drosophila with tRNA-flanked sgRNAs. Nat Methods. 2016 Oct;13(10):852-4.

39. Xie K, Minkenberg B, Yang Y. Boosting CRISPR/Cas9 multiplex editing capability with the endogenous tRNA-processing system. PNAS. 2015 Mar 17;112(11):3570-5.

40. Qi W, Zhu T, Tian Z, Li C, Zhang W, Song R. High-efficiency CRISPR/Cas9 multiplex gene editing using the glycine tRNA-processing system-based strategy in maize. BMC Biotechnol. 2016 Dec;16(1):58.

41. Hartmann RK, Gößringer M, Späth B, Fischer S, Marchfelder A. Chapter 8 The Making of tRNAs and More - RNase P and tRNase Z. In: Progress in Molecular Biology and Translational Science [Internet]. Academic Press; 2009 [cited 2019 Nov 19]. p. 319-68. (Molecular Biology of RNA Processing and Decay in Prokaryotes; vol. 85). Available from: http://www.sciencedirect.com/science/article/pii/S0079660308008088

42. Hoyle HD, Hutchens JA, Turner FR, Raff EC. Regulation of $\beta$-tubulin function and expression in Drosophila spermatogenesis. Developmental Genetics. 1995;16(2):148-70.

43. Kandul NP, Liu J, Sanchez C. HM, Wu SL, Marshall JM, Akbari OS. Transforming insect population control with precision guided sterile males with demonstration in flies. Nat Commun. 2019 Dec;10(1):84.

44. White-Cooper H. Tissue, cell type and stage-specific ectopic gene expression and RNAi induction in the Drosophila testis. Spermatogenesis. 2012 Jan 1;2(1):11-22.

45. Wakiyama M, Matsumoto T, Yokoyama S. Drosophila U6 promoter-driven short hairpin RNAs effectively induce RNA interference in Schneider 2 cells. Biochemical and Biophysical Research Communications. 2005 Jun 17;331(4):1163-70.

46. Turkel R. Development of repressible sterility in Medfly for chemical-free pest control [Internet] [phd]. Cardiff University; 2016 [cited 2019 Nov 17]. Available from: http://orca.cf.ac.uk/99253/

47. McKearin DM, Spradling AC. bag-of-marbles: a Drosophila gene required to initiate both male and female gametogenesis. Genes Dev. 1990 Dec 1;4(12b):2242-51. 


\section{Figures:}

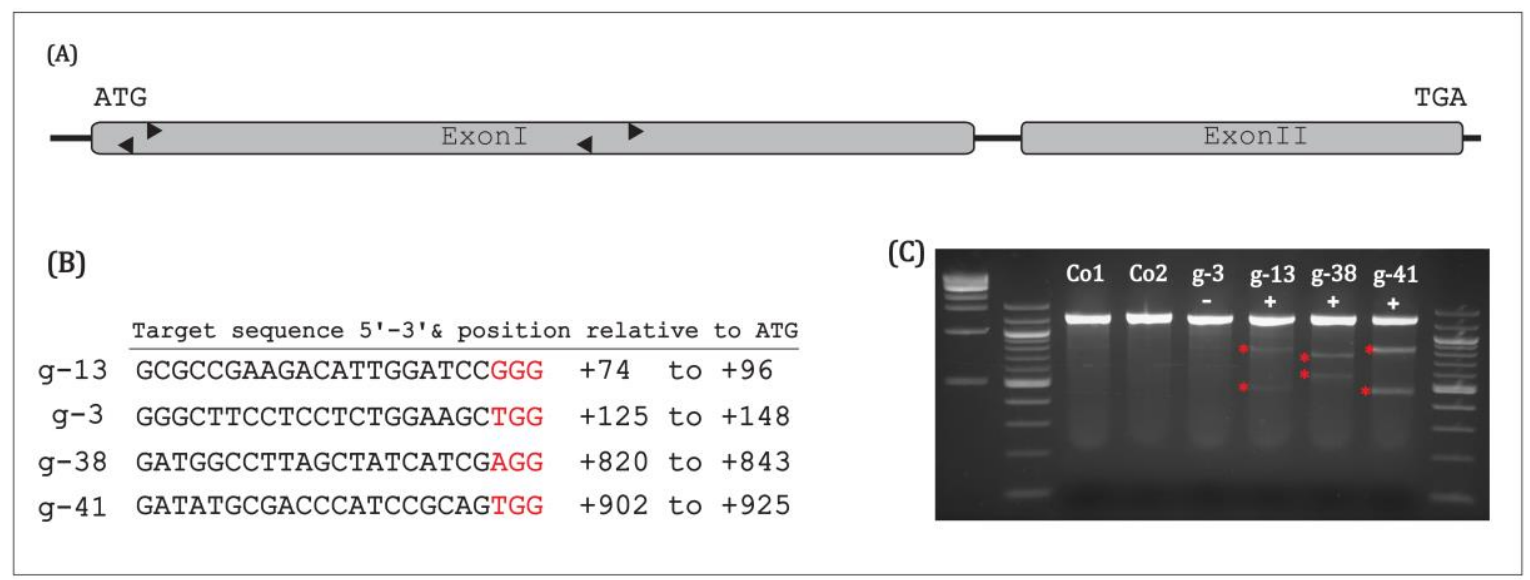

Figure. 1. Identification and validation of guide RNAs targeting Dm sneaky gene. (A) the structure of Dm snky has two exons and one intron. Four $g R N A s$ were identified. Solid black triangles indicate the relative position and the strand for each gRNA relative to each other and to the translation start codon. (B) sequence of each target with the PAM shown in red. The relative position relative to the first nucleotide of the translation start codon is shown. (C) agarose gel picture showing the result of T7 Endo assay. g-13, g-38 and g-41 are positive whereas g-3 was not digested by T7Endo and therefore considered negative. Co1 is T7 Endo-less negative control for g- 3 and g13 while Co2 control for g-38 and g-41 
(A)

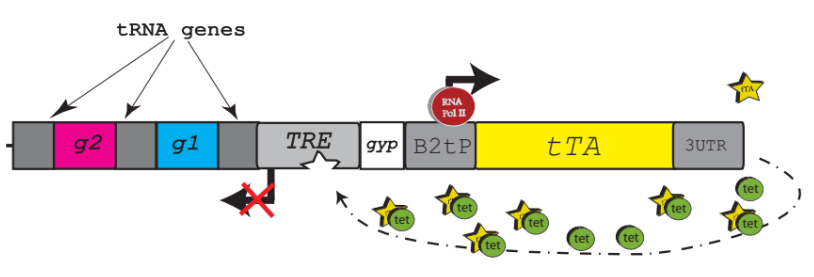

(B)

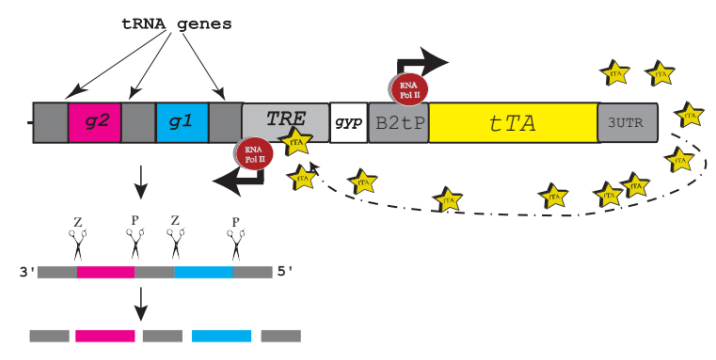

(C)

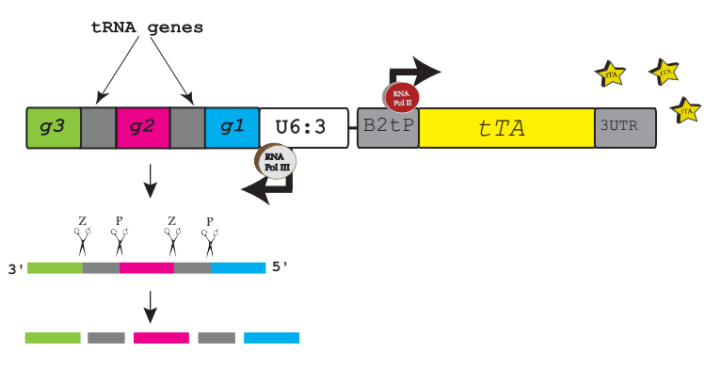

Figure. 2. Illustration of the gRNA-driver constructs. (A) shows the different components of the gRNA-driver construct in which the $t R N A$ processing strategy was adopted to express two $g R N A s$ simultaneously. The $g R N A s$ are flanked and interspaced by $t R N A$ which should facilitate correct processing of $g R N A s$. The second part of the construct is the $t T A$ under the control of $\mathrm{E} / \mathrm{P}$ of $D$. melanogaster $\beta 2 t$ gene. In the presence of tetracycline in the fly food, the tet bind the tTA and prevent it from binding to the tTA responsive element (TRE) and as a result the $g R N A$ cassette will not be expressed. (B) in the absence of tet in the food, the tTA is free to bind to the TRE and direct the expression of the $g R N A s$ cassette in the testes. The transcript will then be processed to individual $g R N A$ by the cell ribonuclease $\mathrm{P}$ and $\mathrm{Z}$. having the $T R E$ in the same construct close to driver construct can lead to overexpression of $t T A$ by a positive autoregulatory loop which may lead to unintended cytotoxicity $(\mathbf{C})$ As a second strategy for constitutive expression of $g R N A s$ we used the promoter of $D$. melanogaster small nuclear $R N A$ gene $U 6: 3$ to drive three $g R N A$ s by utilizing the $t R N A$ processing system described in $\mathrm{B}$. 


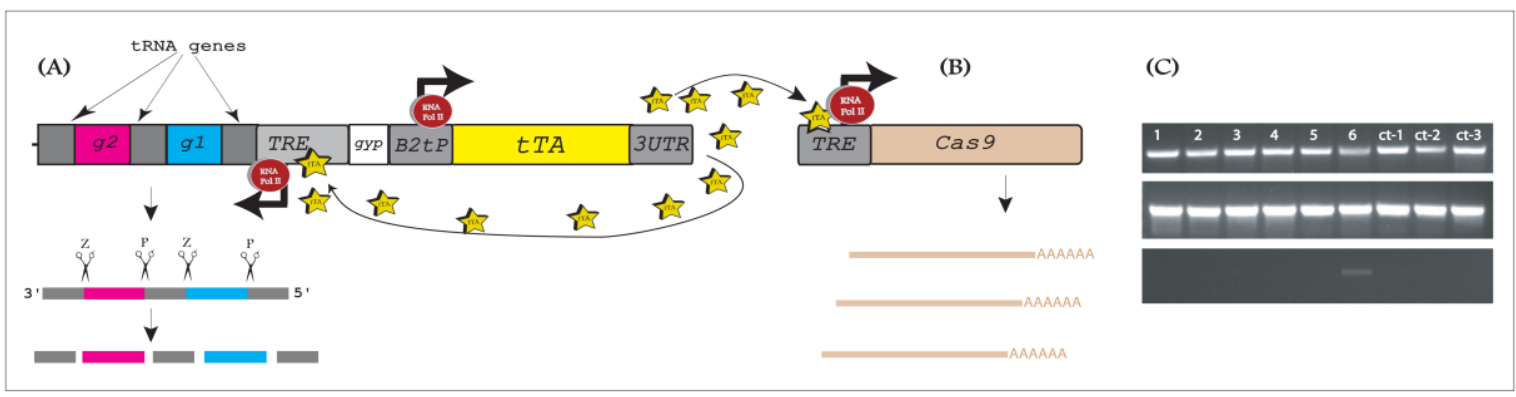

Figure. 3. Transactivation of Cas9 and gRNAs expression during spermatogenesis (A) Illustration of the two components gRNA-driver construct in which the expression of two $g R N A$ from $R N A$ polII promoter is controlled by the tTA and $\beta 2 t$ promoter. In the absence of tetracycline, tTA binds and activates the expression of two gRNAs interspaced and flanked by three tRNAs genes to facilitate correct processing into individual gRNAs by the action of the cell ribonuclease $\mathrm{P}$ and Z. despite incorporation of the gypsy insulator between the gRNAs cassette and the spermatogenesis-specific driver construct, the tTA can potentially overexpress itself as a result of a positive feedback loop due to its binding to the $t T A$ responsive element (TRE). (B) shows the expression of Cas9 under the control of TRE directed by spermatogenesis-specific expression of the $t T A$. (C) the upper panel of the gel picture shows that Cas 9 is expressed in the testes of double heterozygous D. melanogaster strain harboring the two components of the paternal effect embryonic lethality system PEEL shown in A and $\mathrm{B}$. each of the chosen three responder lines was crossed individually to two gRNA-driver line. Each two consecutive numbers represent one responder line crossed to driver lines 372_M14_M1 and 372_F26_F1 respectively. The last three lanes are RT-PCR negative control using the respective responder line not crossed to any driver. $\mathrm{Ct}-1$ controls for 1 and 2 , ct- 2 controls for 3 and 4 , and ct 3 controls for 5 and 6 . Ideally the negative controls should not express Cas 9 . However, basal promoters can drive expression at a basal level depends on the position of integration. The middle lane is the positive control for the RT-PCR procedure using primer pair to amplify from the same cDNA pool the 3'UTR of the spermatogenesisspecific $\beta 2 t$ gene. All of the lanes gave strong bands which reflect the reliability of the tests, especially when looking at the result on the light of the lower lane which represent the RT-less PCR following the same procedure and the same amount of the initial RNA but without addition of the reverse transcriptase. Cas9 expression in the negative non-crossed control in the upper doesn't not necessarily means that the responder lines are not functional due to leakiness, but the PCR is highly sensitive and can detect low levels of expression from very few molecules. 


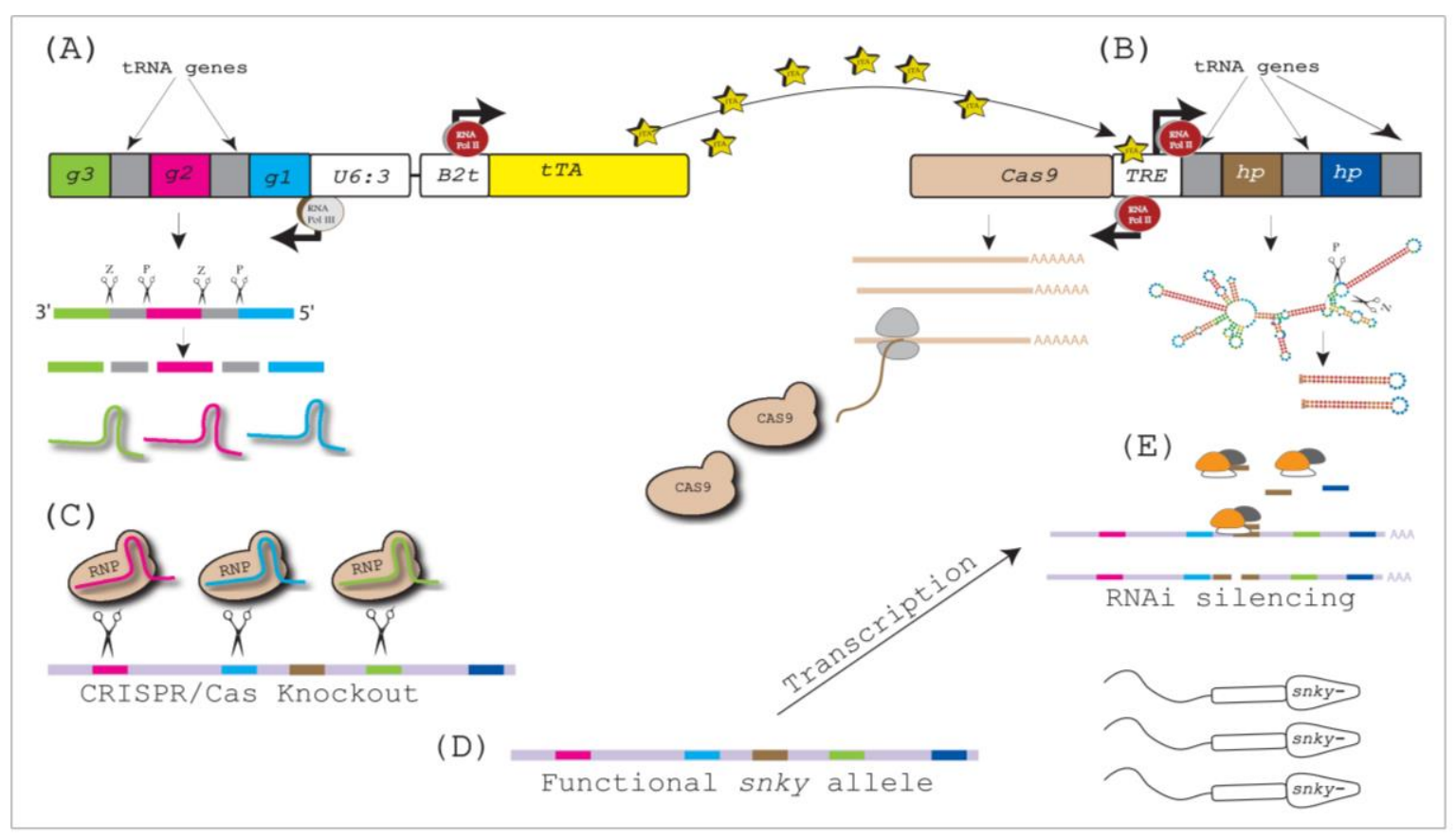

Figure.4. Two conditional mechanisms for paternal effect embryonic lethality system. (A) Depiction of a two components gRNA-driver construct where the RNA polIII transcribes from the promoter of the $s n R N A$ gene $U 6: 3$ three gRNAs $g 1, g 2$ and $g 3$ shown in blue, lila and green respectively, targeting the PEEL gene snky. The $g R N A s$ are interspaced by two $t R N A$ genes to ensure correct processing by the cell ribonucleases $\mathrm{P}$ and $\mathrm{Z}$ (shown as scissors) to individual $g R N A s$. The second part of the construct shows the expression of the heterologous transcription factor tTA driven by the promoter of the gene $\beta 2 t$ during spermatogenesis. (B) Illustrates the conditional expression of the two effectors, Cas 9 and shRNA $(h p)$ against the PEEL gene snky. In the absence of tetracycline in the fly's food, the tTA is allowed to bind to its responsive element (TRE) placed between the two effector cassettes and since the TRE is bidirectional it should allow expression of the two effectors at the same time. Cas9 is expressed and translated early before meiosis. The second effector transcribes two hairpins ( $h p 1$ and $h p 2$ depicted in brown and blue respectively) interspaced and flanked by three $t R N A$ genes to facilitates correct processing of the hairpins by the ribonucleases $\mathrm{P}$ and $\mathrm{Z}$. The targets of the two hairpins in snky mRNA are different from CRISPR genomic targets (C) Cas9 forms RNP complexes with the constitutively expressed $g R N A s$ against snky, and therefore will be guided to the respective target and induce simultaneously three DSBs which should lead to efficient knockout of the gene. (D) Despite early knockout of the gene, some snky mRNA might have already been transcribed, or some resistant alleles are naturally available or formed as a result of NHEJ repair of the DSBs that can still be functional. (E) illustrates triggering of the RNAi pathway by the two hairpin RNAs to ensure depletion of snky mRNA if available. This double action effect should provide a tight filter system to produce $100 \%$ sterile males with competent sperm that are snky and thus should fail to form functional male pronucleus during fertilization and as a consequence embryonic development will be arrested. 


\section{Materials and Methods}

\section{Drosophila strains}

All fly experiments were performed in our well-equipped safety level one (S1) laboratory, which is certified for generating and using genetically modified insects.

Wild type $D$. melanogaster Oregon $\mathrm{R}$ strain as well as the transgenic flies generated during this study were reared on standard Drosophila food and kept at $25^{\circ} \mathrm{C}$ throughout this study.

\section{Nucleic acid isolation}

Genomic DNA was isolated from adult males and females using NucleoSpin ${ }^{\circledR}$ DNA Insect (Macherey-Nagel) according to the manufacturer instructions. Total RNA was isolated from testes of 4-5 days old males using ZR Tissue \& Insect RNA MicroPrep (Zymo Research Europe, 79110 Freiburg) according to manufacturer instructions.

Unless otherwise indicated all PCR amplifications during this study were performed using Phusion DNA polymerase and Phusion-HF buffer (New England Biolabs GmbH, D-65926 Frankfurt am Main). Primers used are available in supplementary Table 1. Plasmid isolation and PCR purification were performed using NucleoSpin ${ }^{\circledR}$ Plasmid and NucleoSpin ${ }^{\circledR}$ Gel and PCR Clean-up kits (Macherey-Nagel GmbH \& Co., 52355 Dueren, Germany), respectively. NucleoSpin ${ }^{\circledR}$ Plasmid Transfection-grade (Macherey-Nagel) or QIAGEN Plasmid Plus Midi Kit (QIAGEN GmbH, 40724 Hilden, Germany) were used to prepare plasmids for germline transformation.

\section{Sequencing of $D$. melanogaster and $D$. suzukii snky gene}

About 2.k Kb fragment of D. melanogaster snkygene spanning exon I was PCR amplification OreR and w- using primers pair HM\#154 /HM\#156. And program $\left[98^{\circ} \mathrm{C} 3^{\prime \prime}\left(98^{\circ} \mathrm{C} 30^{\prime \prime} 68^{\circ} \mathrm{C}\right.\right.$ $30^{\prime \prime} 72^{\circ} \mathrm{C} 1^{\prime}$ ) $35 \mathrm{X}$ with a final elongation step of $7^{\prime}$ at $\left.72^{\circ} \mathrm{C}\right]$. The amplified DNA fragments were resolved in 1\% agarose gel, documented, purified using PCR and Gel purification kit and sequenced.

To identify $D$. suzukii homologue we used the sequence of $D$. melanogaster as a query to search in $D$. suzukii genome data base http://spottedwingflybase.org/. primer pair HM\#610/HM\#611 were designed and used to amplify 1.7Kb spanning exon I of the potential Ds-snky gene with PCR program $\left[98^{\circ} \mathrm{C} 3^{\prime}\left(98^{\circ} \mathrm{C} 30^{\prime \prime} 68^{\circ} \mathrm{C} 30^{\prime \prime} 72^{\circ} \mathrm{C} 1^{\prime}\right) 35 \mathrm{X}\right.$ with a final elongation step of $7^{\prime}$ at $72^{\circ} \mathrm{C}$. The amplified DNA fragment was resolved in $1 \%$ agarose gel, documented, purified and sequenced

\section{Design of gRNAs targeting Dm-snky}

Based on the obtained sequence for $D$. melanogaster OreR and $\mathrm{w}$ - strains, we used $1 \mathrm{~Kb}$ sequence starting at the ATG translation start codon and searched for gRNA using online 
target finder tool developed by Wisconsin university https://flycrispr.org/.. The following parameters were chosen for the search, target length 20 nucleotides, CRISPR targets with at least one G at the 5', PAM sequence NGG. The obtained targets were evaluated for off-targets based on the latest $D$. melanogaster release r-6 (37) with high stringency. Four targets with zero-off targets were chosen, two of which were close to the ATG translation start codon (Dm_snky_g13 and Dm_snky_g3) and two about 800 to $900 \mathrm{bp}$ downstream of the ATG (Dm_snky_g38, and Dm_snky_g41), table S1.

\section{Constructs:}

To generate plasmids HMMA324 and HMMA325 to conditionally express two $g R N A s$, first a synthetic $312 \mathrm{bp}$ dsDNA fragment containing D. melanogaster Ala:Val:gly was purchased from Integrated DNA Technologies (Integrated DNA Technologies, BVBA, B-3001 Leuven, Belgium) and cloned into pJet1.2 giving rise to HMMA289 which is confirmed by sequencing. The fragment was then digested from HMMA289 using EcoRI/BamHI and cloned into HMMA265 and HMMA266 respectively giving rise to HMMA190 and HMMA191. The Asc fragment containing TREp-Ala:Val:gly:SV40 from HMMA190 and HMMA191 was cloned into HMMA307 Giving rise to HMMA309 and HMMA310 respectively. the CRISPR $g R N A$ scaffold was then PCR amplified from HMMA093 (3.3) using primers pairs HM\#633/\#634HM and HM\#635/\#636HM and cloned into the BsaI and SapI sites respectively of HMMA309 and HMMA310 resulting in HMMA324 and HMMA325 respectively.

To generate plasmid HMMA349 and HMMA410 to constitutively express three gRNA from Dm U6:3 or Ds U6c promoter, the fragment containing the $t R N A$ genes and $g R N A$ scaffolds was PCR amplified from HMMA324 using primer pair HM\#757/HM\#758 and HM\#759/HM\#758 and subsequently cloned by Gibson assembly into Bbs site of HMMA308 and HMMA332 respectively.

To generate gRNA-Driver constructs HMMA371, HMMA372, HMMA373 and HMMA374 expressing tTA and $g R N A s$ targeting Dm-snky, first annealed oligos generating Dm_snky_g13 (HM\#688/HM\#651) and Dm_snky_g38 (HM\#689/HM\#655) were cloned into BbsI and BsaI sites of HMMA324 respectively giving rise to HMMA347, and Dm_snky_g13 (HM\#688/HM\#651) and Dm_snky_g41 (HM\#690/HM\#657) were cloned together into BbsI and BsaI sites of HMMA324 to give rise to HMMA348, and subsequently the $g R N A$ cassette was then PCR amplified from HMMA347 and HMMA348 using primers pair HM\#704/HM\#705, and program $\left(98^{\circ} \mathrm{C} 3^{\prime} 98^{\circ} \mathrm{C} 30^{\prime \prime} 62^{\circ} \mathrm{C} 30^{\prime \prime} 72^{\circ} \mathrm{C} 30^{\prime \prime}\right) 5 x$ followed by $35 \mathrm{X}\left(98^{\circ} \mathrm{C} 30^{\prime \prime} 72^{\circ} \mathrm{C} 1^{\prime}\right)$ and final elongation of $7^{\prime}$ at $72^{\circ} \mathrm{C}$. The fragments were then digested by BsaI and cloned into EcoRI site of HMMA355 and HMMA356 giving rise to HMMA367, HMMA368, HMMA369 and HMMA370. Finally, the Asc fragment from 
HMMA367, HMMA368, HMMA369 and HMMA370 containing the gRNA and driver cassette were cloned into the Asc site of HMMA331 (chapter 3.4).

To generate plasmids HMMA355 and HMMA356, the gypsy insulator was PCR amplified from mfs\#1221 using primer pair HM\#702/HM703, digested by BsaI/BbsI and cloned into EcoRI site of HMMA352 and HMMA353

To generate the dual sperm-marking drive construct HMMA425, HMMA426 and HMMA427, the picornavirus self-cleaving peptide $P 2 A$ was cloned into HMMA131 EcoRI/Ncol sites to give rise to HMMA382. The P2A::DsRed was PCR amplified using primers pair HM\#128/HM\#782 and digested by BsaI. The $t T A$ was amplified using primers HM\#780/HM781 and program $\left[98^{\circ} \mathrm{C} 3^{\prime}\left(98^{\circ} \mathrm{C} 30^{\prime \prime} 63^{\circ} \mathrm{C} 30^{\prime \prime} 72^{\circ} \mathrm{C} 30^{\prime \prime}\right) 35 \mathrm{X}\right.$ with final elongation of $7^{\prime}$ at $72^{\circ} \mathrm{C}$ ] and digested by $B s a \mathrm{I} / \mathrm{XbaI}$. The two fragments were then cloned together into the $X b a I / B a m H I$ sites of HMMA351, HMMA352 and HMMA353. To give rise to HMMA404, HMMA405 and HMMA406. The $t T A: \because P 2 A$ was PCR amplified from HMMA404 using primer pair HM\#780/HM\#69 and program $\left[98^{\circ} \mathrm{C} 3^{\prime}\left(98^{\circ} \mathrm{C} 30^{\prime \prime} 63^{\circ} \mathrm{C} 30^{\prime \prime}\right.\right.$ $\left.72^{\circ} \mathrm{C} 30^{\prime \prime}\right) 35 \mathrm{X}$ and a final elongation of $7^{\prime}$ at $72^{\circ} \mathrm{C}$ ] and digested by $\mathrm{XbaI} / \mathrm{NcoI}$. The $t G F P$ was PCR amplified from mfs\#1256 (32) using primer pair HM\#792/HM\#571, digested by $B b s \mathrm{~B} B \mathrm{BmHI}$ and cloned together with $t T A:: 2 A$ into $X b a \mathrm{I} / B a m H \mathrm{I}$ sites of HMMA352, HMMA352 and HMMA353 giving rise to HMMA407, HMMA408 and HMMA409 respectively. And finally, the AscI fragment from HMMA407, HMMA408 and HMMA409 was cloned into AscI site of HMMA331 (chapter 3.4).

To generate plasmid HMMA350 pSL\{af_DmU6:3_g13_g38_g41_af\} expressing Dm_snky_g13,Dm_snky_g38, and Dm_snky_g41simultaneously from U6:3 promoter, the fragment containing the Dm_snky_g13, Dm_snky_g41 was PCR amplified from plasmid HMMA348 using primer pair HM\#683/HM\#684 and cloned along with annealed oligos HM\#807/HM\#808 introducing Dm_snky_g38 into BbsI site of HMMA332

To finally generate the transformation plasmid HMMA433 and HMMA434, the $g R N A$ cassette under the $U 6: 3$ promoter was PCR amplified from HMMA350 using primers pair HM\#704/HM\#705, digested by BsaI (generates EcoRI compatible ends) and cloned in the EcoRI site of HMMA408 and HMMA409 giving rise to HMMA431 and HMMA432. Eventually the AscI fragment from HMMA431 and HMMA432 was cloned into HMMA331.

To generate Cas9 responder construct HMMA338, the AscI fragment TREp:3XFag:nls:Cas9:nls:SV4O from HMMA327 was cloned into the piggyBac transformation vector HMMA331. HMMA327 is generated by replacement of attP-TREp in HMMA326 by EcoRI/AgeI TREp from HMMA265. HMMA326 was generated by cloning of annealed oligo HM\#102 into ClaI site of HMMA313 which is generated by removal of $D j$ TSE and the ATG between the 3XFlag tag and the nuclear localization signal at the N-terminal of Cas 9 from HMMA295 using BgII (pJet intermediate and mutagenesis 
PCR in supp). HMMA295 was generated by cloning of EcoRI/XbaI fragment form HMMA249 into EcoRI/XbaI sites of HMMA51 (chapter 3.4). HMMA249 was made by cloning of $D j$ TSE into EcoRI site of HMMA194 which is generated by replacement of $D s-$ hsp70 promoter in HMMA056 (chapter 3.3) by attP-TREp EcoRI/ClaI.

All final construct mentioned in this chapter are listed in supplementary table S2.

\section{Generation of shRNA responder construct}

To express two shRNA targeting Dm snky at the same time under the control of $t T A$, we cloned annealed oligos HM\#772/HM\#773 (T13) and HM\#774/HM\#775 (T41) into HMMA309 golden gate to give rise to HMMA430

\section{Germline transformation}

All injections for piggyBac germline transformation were performed using the hyperactive helper plasmid MK006 along with the respective transformation vector at a final concentration of a $500 \mathrm{ng} / \mu \mathrm{L}$ and $300 \mathrm{ng} / \mu \mathrm{L}$ respectively. Emerged $\mathrm{G}_{0}$ flies were crossed individually to three wildtype flies of the opposite sex.

Identification of $F_{1}$ transgenic flies was based on the respective fluorescent body marker. Screening for transgenic flies was performed using Leica M205 FA fluorescence stereomicroscope equipped with camera Q imaging Micropublisher 5.0 RTV (Leica Mikrosysteme Vertrieb Gmb, Wetzlar, 35578 Germany). Transgenic flies were screened using filter sets RFP (excitation: ET546/10x, emission: ET605/70m) or GFP-LP (excitation: ET480/40, emission: ET510 LP). $F_{1}$ transgenic flies were outcrossed to WT flies of the opposite sex.Fly crosses

To test for sterility or partial sterility of the recovered gRNA-driver transgenic lines, 1 males of each strain was individually crossed to $2-3$ virgin wild type females.

About 10 gRNA-driver males from each strain were crossed to 10-15 female Cas9 responder females to test whether the driver lines are functional and can lead to sterility. $15 \mathrm{~F}_{1}$ males of each cross were used for RNA isolation.

\section{RT-PCR}

To investigate whether Cas9 is driven in the testes by the heterologous transcription factor tTA, we performed RT-PCR. Total RNA, was isolated from testes of 15 individual 4-5 days old adult males double heterozygous for the driver and responder transgenes. The males were dissected in ice cold PBS 1X and testes were transferred to bashing tube with beads and fixed on a vortex. Homogenization was allowed to proceed for 10 minutes. And RNA was isolated according to the instructions of the manufacturer. In column DNase treatment was preformed according to the manufacturer instructions. 
In tube DNase treatment was performed using dsDNase provided with the kit. RNA was $1 \mu \mathrm{g}$ was used. First strand cDNA synthesis was done using Maxima First Strand cDNA Synthesis Kit for RT-qPCR, with dsDNase (thermofisher). $2 \mu \mathrm{L}$ cDNA was used for each PCR reaction in a total volume of $25 \mu \mathrm{L}$. to check the expression of cas9, we used primer pair HM\#619/HM\#135 to amplify 460 bp of Cas9. As a negative control the respective Cas9 responder not crossed to driver were used. As a positive control, a pair of primers (HM\#706/HM\#707) that amplify the 3'UTR of b2t was used which give 250bp. The PCR was performed using $2 \mu \mathrm{L}$ cDNA, $1.25 \mu \mathrm{L}$ forward and $1.25 \mu \mathrm{l}$ reverse primer $(10 \mu \mathrm{M}), 2 \mu \mathrm{L}$ dNTPs mix and Phusion polymerase in a total reaction of $25 \mu \mathrm{L}$

$15 \mu \mathrm{L}$ of the PCR product was run in $1.5 \%$ agarose gel and documented using UV lamp and a camera attached to a printer. 
Table S1. Primers sequences

\begin{tabular}{|c|c|c|}
\hline Code & Name & Sequence $5{ }^{\prime}-3^{\prime}$ \\
\hline HМ\#69 & DsRed-mega-R & GGAAGGACAGCTTCTTGTAGTCGGGG \\
\hline HM\#128 & HM_pSL_R & CCGGCTCGTATGTTGTGTGGAATTGTG \\
\hline HM\#135 & cas9-R & GTAGATGGTGGGGTACTTCTCGTGG \\
\hline HM\#154 & Snky_F & GGGATGCAATCAAGGCCACCACTTCTCC \\
\hline HM\#156 & Snky_R & CCGTAGTGGACTCGTGATGGGTTCGAC \\
\hline HM\#571 & HM_tGFP_BamHI_R & CGCTGGATCCTTATTCTTCACCGGCATCTGCATCC \\
\hline HM'610 & HM_Dssnky_F & ATGTTCTCCTTTCTGACGCTGCCATGTC \\
\hline HM\#611 & HM_Dssnky_R & TTATTTCTCTTTCCTGTAGGCGTACACCTC \\
\hline HM\#619 & HM_Cas9_ATG_F & GACGATGACGATAAGGCCCCAAAGAAGAAGCGGAAGGTC \\
\hline HM\#633 & HM_BsaI_Scaf_BbsI_F & CATGGTCTCCTCCAGGGTCTTCGAGAAGACCTG \\
\hline HM\#634 & HM_BsaI_Scaf_BbsI_R & ACGGGTCTCGAAACGCACCGACTCGGTGCCACTTTTTC \\
\hline HM\#635 & HM_SapI_Scaf_BsaI_F & GGGGCTCTTCAACAGAGAGACCGAGAGAGGGTCTCAG \\
\hline HM\#636 & HM_SapI_Scaf_BsaI_R & CCGGCTCTTCACGCGCACCGACTCGGTGCCACTTTTTC \\
\hline HM\#650 & HM_Dmsnkyg13_F & CGTCGCGCCGAAGACATTGGATCC \\
\hline HM\#651 & HM_Dmsnkyg13_R & AAACGGATCCAATGTCTTCGGCGC \\
\hline HM\#652 & HM_Dmsnkyg3_F & CGTCGGGCTTCCTCCTCTGGAAGC \\
\hline HM\#653 & HM_Dmsnkyg3_R & AAACGCTTCCAGAGGAGGAAGCCC \\
\hline HM\#654 & HM_Dmsnkyg38_F & CGTCGATGGCCTTAGCTATCATCG \\
\hline HM\#655 & HM_Dmsnkyg38_R & AAACCGATGATAGCTAAGGCCATC \\
\hline HM\#656 & HM_Dmsnkyg41_F & CGTCGATATGCGACCCATCCGCAG \\
\hline HM\#657 & HM_Dmsnkyg41_R & AAACCTGCGGATGGGTCGCATATC \\
\hline HM\#683 & HM_gBlk_U6:3_F & GCGAGAAGACTACGTCGGGGATGTAGCTCAGATGGTAGAG \\
\hline HM\#684 & HM_gBlk_U6:3_R & CAAGAAGACCTTGCGTCGGCCGGGAATCG \\
\hline HM\#688 & HM_Dmsnkyg13_F2 & TCCAGCGCCGAAGACATTGGATCC \\
\hline HM\#689 & HM_Dmsnkyg38_F2 & AACAGATGGCCTTAGCTATCATCG \\
\hline HM\#690 & HM_Dmsnkyg41_F2 & GCAGATATGCGACCCATCCGCAG \\
\hline HM\#702 & HM_EcoRI_gypsy_F & GGCGAAGACCGAATTCGATCCGGCTAAATGGTATGGCAAGAAAAG \\
\hline HM\#703 & HM_EcoRI_gypsy_R & CGAGAAGACCGAATTAGGCCCGGTACCCTATTCGCAAAAACATTG \\
\hline HM\#704 & HM_Bsa_EcoR_TRE_F & TAGGTCTCGAATTCGCCGGCCGAATTTCGAGTTTACC \\
\hline HM\#705 & HM_EcoRI_SV40_F & CGCGGTCTCGAATTCAAGCTTGATACATTGATGAGTTTGGAC \\
\hline HM\#706 & HM_b2t_3UTR_F & CGAGGATCCTAGGATTAACTTCCCACTCAAGATCACACATG \\
\hline HM\#707 & HM_b2t_3UTR_R & GCCAAGCTTGTCTGCTTATAAATCAACATTTATTCGTAACCC \\
\hline HM\#757 & HM_U6c_gib_F & $\begin{array}{l}\text { ATATACGACATTTTTCAATACGAAATCGGGGATGTAGCTCAGATGGTAGA } \\
\text { GCGCTC }\end{array}$ \\
\hline HM\#758 & HM_U6c_gib_R & $\begin{array}{l}\text { ACTTGCTATTTCTAGCTCTAAAACAGAAGAGCACGCTCTTCCTGCGTCGG } \\
\text { CCGGGAATCGAACC }\end{array}$ \\
\hline HМ\#759 & HM_U6:3_gib_F & $\begin{array}{l}\text { TAGACCTATTTTCAATTTAACGTCGGGGATGTAGCTCAGATGGTAGAGCG } \\
\text { CTC }\end{array}$ \\
\hline HM\#772 & HM_DmT13sh_F & $\begin{array}{l}\text { TCCACGGATTTAATGTCTTCGGTGCCGAATAATTCGGATCCAATGTCTTC } \\
\text { GGCGC }\end{array}$ \\
\hline HM\#773 & HM_DmT13sh_R & $\begin{array}{l}\text { AAACGCGCCGAAGACATTGGATCCGAATTATTCGGCACCGAAGACATTAA } \\
\text { ATCCG }\end{array}$ \\
\hline HМ\#774 & HM_DmT41sh_F & $\begin{array}{l}\text { ACAGATATGTGACTCATCTGTAGTCGAATAATTACTGCGGATGGGTCGCA } \\
\text { TATC }\end{array}$ \\
\hline HM\#775 & HM_DmT41sh_R & $\begin{array}{l}\text { CGCGATATGCGACCCATCCGCAGTAATTATTCGACTACAGATGAGTCACA } \\
\text { TATC }\end{array}$ \\
\hline
\end{tabular}




\begin{tabular}{|c|c|c|}
\hline HM\#780 & HM_XbaI_tTA_F & AAGATGTCTAGATTAGATAAAAGTAAAGTGATTAACAGC \\
\hline HM\#781 & HM_BsaI_tTA_F & CCGGTCTCCACCCCCCACCGTACTCGTCAATTCCAAG \\
\hline HM\#782 & HM_BsaI_DsRed_R & GCCGGTCTCGGATCCCTACAGGAACAGGTGGTGGCGGCC \\
\hline HM\#792 & HM_tGFP_F & GCGAAGACATCATGGAGAGCGACGAGAGCGG \\
\hline HM\#807 & HM_Dmsnkyg38_F3 & GCAGATGGCCTTAGCTATCATCG \\
\hline HM\#808 & HM_Dmsnkyg38_R3 & AACCGATGATAGCTAAGGCCATC \\
\hline
\end{tabular}


Table S2. List of vectors

\begin{tabular}{|c|c|}
\hline Code & components of the construct \\
\hline HMMA324 & pSL $\{a f$ TREp_Ala::chiRNA::Val::chiRNA::Gly::SV4O_af\} \\
\hline HMMA325 & pSL $\{$ af_TREhs43_Ala::chiRNA::Val::chiRNA::Gly::SV40_af $\}$ \\
\hline HMMA349 & $\operatorname{pSL}\left\{a f \_U 6: 3 \_\right.$Ala::chiRNA::Val:chiRNA::Gly::chiRNA::DSE_af\} \\
\hline HMMA410 & pSL $\{$ af_U6c_Ala::chiRNA::Val::chiRNA::Gly::chiRNA::DSE_af $\}$ \\
\hline HMMA371 & 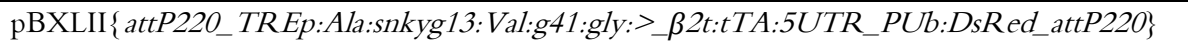 \\
\hline HMMA372 & pBXLII $\{$ attP220_TREp:Ala:snkyg13:Val:g38:gly:>_B2t:tTA:5UTR_PUb:DsRed_attP220\} \\
\hline HMMA373 & pBXLII $\{$ attP220_TREp:Ala:snkyg13:Val:g41:gly: >_DjE/P:tTA:5UTR_PUb:DsRed_attP220\} \\
\hline HMMA374 & pBXLII \{attP220_TREp:Ala:snkyg13:Val:g38:gly:>_DjE/P:tTA:5UTR_PUb:DsRed_attP220\} \\
\hline HMMA430 & pSL $\{$ af_TREp_Ala::snkyshRNAT13::Val::snkyshRNAT41::Gly::SV40_af\} \\
\hline HMMA433 & pBXLII $\left\{a t t P 220 \_U 6: 3: s n k y g 13: g 41: g 38 \_\beta 2 t: t T A: P 2 A: t G F P: 5 U T R \_P U b: D s R e d \_a t t P 220\right\}$ \\
\hline HMMA434 & pBXLII $\left\{a t t P 220 \_U 6: 3: s n k y g 13: g 41: g 38 \_\beta 2 t: t T A: P 2 A: t G F P: 5 U T R \_P U b: D s R e d \_a t t P 220\right\}$ \\
\hline HMMA338 & pBXLII $\left\{a t t P 220 \_T R E p: C a s 9 \_P U b: E G F P \_a t t P 220\right\}$ \\
\hline HMMA425 & pBXLII $\left\{a t t P 220 \_D s \beta 2 t E / P: t T A: P 2 A: t G F P: 5 U T R \_P U b: D s R e d: S V 40 \_a t t P 220\right\}$ \\
\hline HMMA426 & pBXLII $\left\{a t t P 220 \_D m \beta 2 t E / P: t T A: P 2 A: t G F P: 5 U T R \_P U b: D s R e d: S V 40 \_a t t P 220\right\}$ \\
\hline HMMA427 & pBXLII $\left\{a t t P 220 \_D m D j E / P: t T A: P 2 A: t G F P: 5 U T R \_P U b: D s R e d: S V 40 \_a t t P 220\right\}$ \\
\hline
\end{tabular}




\subsection{Perspective on the combined use of an independent transgenic sexing and a multifactorial reproductive sterility system to avoid resistance development against transgenic Sterile Insect Technique approaches}

This chapter presents and discusses a new perspective for the use of genetic engineering to develop pest control strategies. The idea is inspired by the mechanism of sterility induced by ionizing radiation, when multiple chromosomal breaks cause aneuploidy in the sperm or the sired progeny, which leads to embryonic lethality and thus to reproductive sterility. The proposed system of using CRISPR/Cas9 to mimic the action of radiation by induction of many double strand breaks overcomes the deleterious effect on male fitness associated with ionizing radiation. Furthermore, the chapter discusses the use of two independent binary expression systems, the tet-off and the $Q$-system, to enable combing the female-specific embryonic lethality established previously for a number of insect pests as a sexing mechanism with the proposed reproductive sterility system. This should provide then a complete set of independent SIT tools in one system.

Kolja N Eckermann, Stefan Dippel, Mohammad KaramiNejadRanjbar, Hassan M.M. Ahmed, Ingrid M Curril, Ernst A Wimmer*

Authors contributions:

All authors contributed to the manuscript by discussion of the ideas and by revising the text. EAW wrote the first draft and KNE prepared all Figures.

Status of manuscript: Published in BMC Genetics in 2014

DOI: $10.1186 / 1471-2156-15-S 2-S 17$ 
Perspective on the combined use of an independent transgenic sexing and a multifactorial reproductive sterility system to avoid resistance development against transgenic Sterile Insect Technique approaches

Kolja N. Eckermann ${ }^{1}$, Stefan Dippel ${ }^{1}$, Mohammad KaramiNejadRanjbar ${ }^{1}$, Hassan M. M. Ahmed ${ }^{1}$, Ingrid M. Curril $^{1}$, Ernst A. Wimmer ${ }^{1 \$}$

${ }^{1}$ Georg-August-University Göttingen, Johann-Friedrich-Blumenbach-Institute for Zoology and Anthropology, Dept. of Developmental Biology, GZMB, Ernst-Caspari-Haus, Justus-von-Liebig-Weg 11, 37077 Göttingen, Germany

SCorresponding author: E. A. Wimmer. Tel +495513922888.

E-Mail address: ewimmer@gwdg.de (E.A. Wimmer)

\title{
Keywords
}

CRISPR; cas9; Genetically modified insect; genetically modified organism (GMO); insect control; insecticide resistance; insect pest management; molecular entomology; quinic acid

\begin{abstract}
Background

The Sterile Insect Technique (SIT) is an accepted species-specific genetic control approach that acts as an insect birth control measure that can be improved by biotechnological engineering to facilitate its use and widen its applicability. First transgenic insects carrying a single killing system have already been released in small scale trials. However, to evade resistance development to such transgenic approaches, completely independent ways of transgenic killing should be established and combined.
\end{abstract}

\section{Perspective}

Most established transgenic sexing and reproductive sterility systems are based on the binary tTA expression system that can be suppressed by adding tetracycline to the food. However, to create 'redundant killing' an additional independent conditional expression system is required. Here we present a perspective on the use of a second food-controllable binary expression system - the inducible Q system - that could be used in combination with sitespecific recombinases to generate independent transgenic killing systems. We propose the combination of an already established transgenic embryonic sexing system to meet the SIT requirement of male-only releases based on the repressible tTA system together with a redundant male-specific reproductive sterility system, which is activated by Q-system controlled site-specific recombination and is based on a spermatogenesis-specifically expressed endonuclease acting on several species-specific target sites leading to chromosome shredding. 


\section{Conclusions}

A combination of a completely independent transgenic sexing and a redundant reproductive male sterility system, which do not share any active components and mediate the induced lethality by completely independent processes, would meet the 'redundant killing' criteria for suppression of resistance development and could therefore be employed in large scale longterm suppression programs using biotechnologically enhanced SIT.

\section{Background}

Many insects heavily damage agriculture and forestry or transmit deadly diseases to animals and humans. Current control efforts still mostly rely on the use of insecticides, but chemical control is not always harmless and the costs of developing new chemical compounds to overcome the world-wide threat of insecticide resistance are escalating [1]. Moreover, to protect biodiversity the establishment of pest-specific management methods is desirable. The Sterile Insect Technique (SIT) is a species-specific genetic control approach that acts as an insect birth control measure, which relies on the mass rearing, sterilization and field release of large numbers of insects. The competition between released sterile and resident males for mating with wild females leads to the reduction of the reproductive potential. If continued releases of high-quality sterile males in inundating numbers over several consecutive generations are performed, a progressive reduction of the population size and eventually the total eradication of the pest population will occur [2,3]. SIT is now an accepted component of various integrated approaches to control, suppress, prevent, or even eradicate invasive insect pest species from islands, large fruit production areas, or even complete continents [4]. Classically, both male and female insects were released, particularly because the distinction between male and female pupae is hardly manageable or requires the development of genetic sexing strains [5]. Released females, however, although sterile, sting fruits with their ovipositors or keep blood feeding and potentially transmit diseases as well as compete against wild females for mating with the sterile males [5]. In addition, sterilization is classically achieved by irradiation, a procedure that often renders insects very weak and unfit to compete with the wild mates [6]. Such drawbacks and several years of experience have put forward several key requirements for an efficient SIT application: intensive rearing of large numbers of insects for mass release, the availability of efficient sex-separation methods, sterilization techniques able to produce large numbers of insects with minimal effects on fitness and competitiveness, effective release methods, and efficient marking systems to identify released individuals.

Biotechnological engineering of insects makes novel approaches possible to efficiently mark insects as well as selectively produce vigorous and potent sterile males, which are generated by conditional male reproductive sterility in combination with conditional female lethality. This will improve efficacy and widen applicability to further insect pest species $[7,8]$. To minimize 
the concerns coupled with the release of transgenic organisms, SIT programs are actually ideal, as the sterility of the released males will serve as a biological safety mechanism for containment as it impedes the spread of transgenes and allows for a safe deployment $[9,10]$.

In accordance to this hope for novel successful genetic pest management strategies, the first biotechnologically engineered designer insects have already been released in small scale trials: pink bollworm moths in Arizona, USA [11], as well as yellow fever mosquitoes in the Grand Cayman Islands [12], Malaysia [13], with a currently ongoing release in Brazil [14,15]. For the release in the Grand Cayman Islands, it has been shown that the sustained release of transgenic mosquitos carrying a dominant lethal gene could successfully suppress a field population [16] demonstrating the great potential of transgenic SIT approaches. Envisioning the beneficial future use of genetically modified insects, the European Food Safety Authority has recently published a scientific opinion on the guidance on the environmental risk assessment of genetically modified animals including insects [17]. Since reproductive sterility based on lethality systems serves as an intrinsic containment against vertical transmission of transgenes in biotechnologically engineered SIT, its application does not present real concerns in respect to humans and the environment [18].

Nonetheless, the use of transgenic SIT approaches is still at initial stages and an ongoing largescale use somewhat premature, as potential resistance development might pose a significant threat to the further use of this technology [19]. In the currently released transgenic mosquitoes, the dominant lethality is mediated by the overexpression of a synthetic transcription factor that is deleterious to cells at very high levels reached by auto-activation in a positive feedback loop [20]. This presents just one single killing system based on an unclear mechanism. Since most pest insects produce large numbers of offspring, they have a high propensity to evolve resistance to control measures. Actually, classic SIT based on sterilization by radiation is an exception in the resistance development context, as the radiation-induced breaks of the chromosomes are random and vary among all individuals thus providing builtin redundancy [21]. However, transgenic SIT approaches will have defined killing systems that are in principle susceptible to resistance development. Thereby, we assume that the released insects still contain functional transgenes and are themselves susceptible to the dominant lethality [22]. The potential break-down of transgenic characters during mass rearing is an important but different issue for quality control before release. In respect to resistance development the heterogeneous genomes of the field populations are important [21], which might contain genotypes that lead to suppression or partial suppression of the lethality traits. For the avoidance of behavioural resistance, where wild type insects reject mass-reared insects as mating partners, regular introgression of wild type genetic material into the mass rearing strains has been successful [2]. However, there is also the possibility of biochemical resistance to biotechnologically engineered lethality. Due to the inundation of the population with susceptible alleles by the release of the sterile insects during an ongoing 
SIT program, only strong resistance-mediating alleles acting dominant and having only low fitness costs propose a threat to SIT programs and are so far only hypothetical [22].

Nevertheless, insects have successfully developed resistance to synthetic chemicals as well as to microbial agents [23], and are also likely to develop resistance to transgenic SIT approaches when employed in long-term suppression programs [24]. One strategy to significantly impede or at least delay resistance development could be based on the principle of 'redundant killing' $[25,26]$. Therefore, transgenic SIT strains with effective and necessary sterility or lethality traits should only be considered in large scale long-term suppression programs, once completely independent toxicity systems have been combined. Since actually two traits are favourably introduced by transgenesis - female lethality for male only releases as well as reproductive sterility by dominant lethal transgenes - one task is to identify two completely independent ways of mediating them.

\section{Perspective}

\section{Combination of two independent systems: male reproductive sterility and female lethality}

A sterile insect in the sense of SIT is defined as "an insect that, as a result of a specific treatment, is unable to reproduce" [27]. A first approach to cause such reproductive sterility by biotechnological engineering was successfully demonstrated in the non-pest insect $D$. melanogaster [28]. The system is based on the transmission of a transgene combination that causes conditional embryo-specific lethality in the progeny without larval hatching and has successfully been transferred to tephritid fruit flies [29,30]. This prevents larval damage to fruits and the introgression of transgenes into wild type fruit fly populations. Furthermore, for tephritid fruit flies and mosquitoes, transgenic strains were produced using an autocidal overexpression loop of the protein tTA, which leads to dominant lethality when transgenic males were mated to wild type females [20, 31]. Additional transgenic reproductive sterility systems [32,33] might be based on species-specific homing endonucleases [34].

To generate transgenic sexing systems, female lethality was first developed and tested in $D$. melanogaster and based on the female-specific expression of conditional lethal genes $[35,36]$. More recently transgenic sexing systems for tephritid fruit flies have been generated using a female-specifically spliced intron from the transformer gene. First it was used in an autocidal expression loop with the female lethality occurring at late larval stages in the Medfly Ceratitis capitata [37]. This system has successfully been transferred to other Tephritids such as the olive fly Bactrocera oleae [38] and also to blowflies [39] - devastating pests of livestock - as well as to lepidopterans [40]. Furthermore, embryonic transgenic sexing systems have combined the use of such a female-specifically spliced intron with an early embryonic expression mediated by cis-regulatory elements from early acting cellularization genes that indirectly and 
controllably drive the expression of a hyper-active pro-apoptotic gene (Figure 1) [41,42]. An even better understanding of the sex differentiation pathway in insects will provide us with additional strategies for synthetic genetic-based tools for large scale sex separation in SIT applications [43] based on either female killing or actual female sex-reversal $[44,45]$.

\section{tTA: the commonly used conditionally repressible expression system}

The conditionality of the so far established transgenic sexing and reproductive sterility systems is based on a binary expression system, which can be suppressed by supplementing the food with tetracycline (Figure 1). The tetracycline-controlled transactivator ( $t T A)$ consists of a bacterial-viral fusion protein [46] that activates gene expression after binding to a $t T A$ response element (TRE). The major advantage of this binary expression system is that a food supplement can suppress the activation providing an additional control to the directed gene expression [47]. tTA complexed with tetracycline is prevented from binding to its response element and the downstream gene is not activated. The expression system is thus switched off by supplementing the food with tetracycline which allows for an additional control on top of the tissue-specific promoter driving $t T A$ expression. Since only small amounts of tetracycline are needed to suppress the expression, this system has become the most favorable expression system to develop transgenic SIT approaches. However, to create a situation of 'redundant killing' based on two completely independent mechanisms to mediate sterility or female lethality, an additional conditional expression system is necessary.

\section{Second food-controllable expression system: $Q$ system}

Recently a second food-additive controllable expression system - the Q system - has been shown to work ex vivo in mammalian cells as well as in vivo in the vinegar fly $D$. melanogaster $[48,49]$. The broad applicability of this system is also demonstrated by its functionality in the nematode worm Caenorhabditis elegans [50]. The Q system is based on the regulatory genes of the gene cluster qa from the bread mold Neurospora crassa, which allows the fungus to utilize quinic acid as a carbon source [51]. Quinic acid can be found in high concentrations both in herbaceous plants as well as conifers [52] and at especially high levels in unripe fruits [53]. Several molds are able to use quinic acid as carbon source and have specific gene clusters for the catabolic pathway [54]. The regulatory genes of the cluster ensure that the catabolic enzymes are only expressed at the presence of quinic acid: one gene, $q a-1 F(Q F)$, acts as DNA-binding transcriptional activator of all cluster genes, whereas another regulatory gene, qa-1S(QS), acts as a repressor that does not bind DNA itself but inactivates the activator QF by complex formation [54]. Quinic acid acts as an inducer by hindering the repressor QS from complexing QF, which then can activate its target genes (Figure 2). Therefore, the Q system is actually an inducible binary expression system with the food additive, quinic acid, leading to the activation of controlled gene expression. This and the fact that quinic acid is found widespread in nature [52] do not allow us to use this system in an analogous way to the tTA 
system. However, it offers a completely independent expression system that should be utilized for novel transgenic SIT approaches.

\section{Render inducible system suitable for transgenic SIT approaches}

An inducible system would usually require that the inducer is constantly present to have the system activated. But as this cannot be warranted for a food-additive after release, a temporary induction of the system needs to be stabilized into a continuous expression. For this purpose, site-specific recombination systems [55] can be utilized to stabilize an inducer pulse into a persistent activation. For the $f p$ recombinase (FLP) it was demonstrated in D. melanogaster that a region-specific promoter can be separated from the downstream coding region by a $f p$ out cassette that contains a transcriptional terminator and is flanked by $f l p$ recombinant target sites (FRTs) [56,57]. The transcriptional terminator prohibits the directed expression mediated by the tissue-specific promoter until FLP removes the flp-out cassette by sitespecific recombination of the FRTs that are in direct orientation (Figure 2). The left over single FRT in the 5'UTR does not interfere with effective transcription and translation of the downstream coding sequences [56,57]. On this basis, the Q binary system can be combined with the FLP mediated transcriptional activation system to stably activate the expression of a gene after a pulse induction with an inducer (Figure 2).

To reduce the number of constructs necessary for such a complex inducible $Q$ and immediate targeted gene expression system, actually the regulatory components of the $\mathrm{Q}$ system can be placed into the $f l p$-out cassette (Figure 2) which will also place the $\mathrm{Q}$ system components under the same control as the later expressed effector gene [57]. To actually place both regulator genes - QF and QS - into the same construct, the two coding regions can be separated by an internal ribosome entry site (IRES) to allow for a bi-cistronic transcript. Depending on the translational start efficiency of the insect virus IRES compared to the actual capped mRNA [58], the QS and QF coding sequences should be placed accordingly to make sure that repressor QS will be in surplus to the activator QF.

In D. melanogaster it has been shown that FLP expression driven by the $\square 2$ tubulin $(\square 2 \mathrm{tub})$ promoter is highly efficient to cause cassette flip-out during spermatogenesis leading to the transmission of the activated effector construct into the next generation [56,57]. Since the $\square 2$ $t u b$ promoter would also enable the generation of reproductive sterility systems [7], this promoter would be very suitable for such a complex system. Respective promoters have already been cloned from a number of different tephritid and mosquito species and functionally used for sperm marking purposes [59,60,61].

To cause reproductive sterility, finally an effector needs to be activated that either causes male sterility by sperm depletion, e.g. by expression of a cell death gene or a cell-specific toxin that is active in the cytoplasm only and has no trans-membrane movement abilities to protect 
adjacent tissue or predatory organisms [7,61]. However, as such sterile males would not transfer sperm to females, such females would continue to search further for sperm-providing wild type males. Therefore, an effector that would kill the progeny but not the sperm would thus be much more suitable. This will allow for sperm development and transfer and therefore renders the females at least temporarily refractory to subsequent mating with wild type males. Such an effector could be a homing endonuclease (Figure 2) that does not affect spermatogenesis - thus producing functional sperm - but attacks the genome of the zygote or prevents the fusion of the male and female pro-nuclei [34]. This would serve as the best reproductive sterility mechanism as it would cause a dominant early embryonic lethality without affecting the sperm itself by stopping the development of the progeny at the very beginning. Moreover, a homing endonuclease would also be independent in its function from the proposed hyperactive pro-apoptotic gene suggested for the sexing system (Figure 1). However, it should be noted that for an applicable transgenic reproductive sterility system, $100 \%$ male sterility needs to be reached, which requires efficient $f l p$ recombinase repression in the absence of quinic acid and its effective induction in the presence of quinic acid as well as strong expression of a highly active homing endonuclease.

\section{Partial redundancy of the female lethality and reproductive sterility systems.}

The described female lethality and reproductive sterility systems will in fact not be fully redundant, as only the female progeny of the released males will indeed have both lethality systems working. In the male progeny only the reproductive sterility providing the homing endonuclease will be active. Thus, rare strong resistance-mediating alleles might be selected in such male progeny and potentially lead to the accumulation of both the resistance allele and the transgenic lethality allele [22]. However, in case of direct linkage between the two lethality systems, which can be achieved by transgene modification based on site-specific recombination [62], the female lethality in the following generation would severely reduce the chance of accumulation of the lethality allele and thus reduce also the selection of the resistance allele. Since only resistant males would survive, they would be immediately outcompeted by the released SIT males [22].

\section{Redundancy in reproductive sterility by an endonuclease causing chromosome shredding.}

Ideally the reproductive sterility system itself should be highly redundant to cause many different lethal mutations similar to the built-in redundancy of radiation-induced sterility [21]. To achieve this, it would be great to have a number of diverse endonucleases or endonuclease target sites causing chromosome shredding [63]. For this, we propose the employment of an endonuclease from the adaptive bacterial immune system using as essential component clustered regularly interspaced short palindromic repeats (CRISPR) [64,65], which allows bacteria to defend themselves against viruses they encountered before by recognizing and 
cutting the viral DNA sequences. For the human pathogen Streptococcus pyogenes, it could be shown that a single endonuclease - CRISPR-associated nuclease 9 (Cas9) is sufficient to cleave the target DNA [66]. Since it was shown that Cas9 can be directed to any 'protospacer' sequence followed by a protospacer-adjacent motif (PAM) that has only two required bases (NGG) [67] by using short guide RNAs (gRNAs) [68], this CRIPSR/Cas9 system has been successfully employed in many model and non-model organisms to generate gene knock-outs and genome editing [69]. Recently a feature article on this emerging technology has discussed possible uses of the CRIPSR/Cas9 system in gene drives to alter wild populations [70].

By transgenic expression of several gRNAs using RNA polymerase III-dependent promoters, such as the $U 6$ snRNA promoter, it has been shown that the Cas 9 endonuclease can actually be targeted to several diverse targets, which can lead to a mutagenesis rate of up to $100 \%$ $[71,72]$. By our proposed use of the $\square 2 \mathrm{tub}$ promoter, Cas 9 will be highly expressed during spermatogenesis and the mRNA still be highly translated during spermiogenesis [73] to expose the sperm chromosomes to high amounts of the endonuclease (Figure 3). To cause chromosome shredding, several guide RNAs can be employed to direct the CRISPR/Cas9 endonuclease to para-centromeric, sub-telomeric, and microsatellite sequences. The induced double strand breaks will lead to large chromosomal aberrations causing aneuploidies that will mediate multifactorial reproductive sterility.

In fact, one of the caveats of the Cas 9 technology - the potential lack of specificity leading to off-target effects [74] - can serve as an additional advantage in the proposed use here, since it might lead to pleiotropic effects harming further genomic loci. Targeting many chromosomal locations will thus provide the intended redundancy bringing the transgene-induced reproductive sterility a step closer to the built-in redundancy of radiation-induced sterility [21].

\section{Conclusions}

The combination of a transgenic sexing system to meet the SIT requirement of male-only releases based on the repressible tTA directed expression system to create female-specific embryonic lethality using a sex-specifically spliced intron and a hyperactive pro-apoptotic gene (Figure 1) together with a reproductive sterility system based on a sperm-specifically expressed endonuclease controlled by the inducible Q-system in combination with sitespecific recombination (Figure 2) seems a promising approach. These two systems would not share any active components and the lethality would be mediated by completely independent processes. Therefore, cross-resistance to both lethality-mediating processes is extremely unlikely and resistance development would require at least two independent gene loci with the likelihood of co-existence and selection being significantly reduced [25]. It should be noted, however, that this redundancy is only partial as only the female progeny of respective released males will have both lethality systems at work. While this will still reduce the 
likelihood of accumulating transgenic lethal alleles and resistance alleles, we propose an additional level of redundancy for the reproductive sterility system using the CRISPR/Cas9 endonuclease system targeting several chromosomal locations to induce chromosome shredding in the sperm (Figure 3).

The insect strains carrying the combined transgenic female lethality and reproductive male sterility systems would be reared on tetracycline containing food to suppress the femalespecific lethality. The male reproductive sterility would not be activated yet, since the repressor QS would keep the system in an OFF state (Figure 4A). The adult flies of the prerelease generation would then be aged on tetracycline-free food (Figure 4B) in order to stop the suppression of the embryonic female-specific lethality in the next generation $[29,41,42]$. The release generation should then be grown also on tetracycline-free larval food in order to keep the embryonic sexing system on to produce males only: in the absence of tetracycline, the synthetic transactivator tTA would activate a hyper-active pro-apoptotic gene that would lead to programmed cell death in the female embryos, as only the female-specific splicing of the transformer intron in this transcript results in the production of an mRNA capable of translating the functional hyper-active pro-apoptotic protein (Figure 4C). The larval food for the release generation would, however, need to contain quinic acid to inactivate the repressor QS, which would then allow the activator QF to induce the expression of the $f p$ recombinase gene, which then in turn would remove the $\mathrm{Q}$ system regulators and activate the expression of the heterologous endonuclease Cas9 during spermatogenesis (Figure 4C). Released males (Figure 4D) would produce sperm with shredded chromosomes leading to lethal aneuploidy in the next generation similar to radiation-induced reproductive sterility without suffering of somatic damages that causes reduced fitness.A transgenic SIT approach using independent lethality systems would meet the 'redundant killing' criteria for suppression of resistance development and could therefore be employed in large scale long-term suppression programs.

\section{Competing interests}

EAW holds a patent on 'Universal Markers of Transgenesis' (United States Patent No. $6,518,481 \mathrm{~B} 1)$.

\section{Authors' contributions}

EAW designed the study and wrote the first draft of the manuscript. KNE created the figures. All authors contributed to the conception of the study as well as critically revised and approved of the manuscript.

\section{Acknowledgements}

The project profited from discussions at the International Atomic Energy Agency funded meetings of the Coordinated Research Projects "The Use of Molecular Tools to Improve the 
Effectiveness of SIT" and "Development and Evaluation of Improved Strains of Insect Pests for SIT". This work was partially supported by the German Academic Exchange Service (DAAD) with a short-term scholarship to IMC. 


\section{References}

1. Pedigo LP: Entomology and Pest Management. $4^{\text {th }}$ ed. Upper Saddle River: Prentice Hall; 2002.

2. Knipling EF: Possibilities of insect control or eradication through the use of sexually sterile males. JEcon Entomol 1955, 48:459-462.

3. Dyck VA, Hendrichs J, Robinson AS: Sterile insect technique - principles and practice in area-wide integrated pest management. Dordrecht, NL: Springer; 2005.

4. World-Wide Directory of SIT Facilities

$($ DIR-SIT $)$

[http://nucleus.iaea.org/sites/naipc/dirsit/SitePages/Home.aspx].

5. Franz G: Genetic sexing Strains in Mediterranean Fruit Fly, an Example for Other Species Amendable to Large-Scale Rearing for the Sterile Insect Technique. In Sterile insect technique - principles and practice in area-wide integrated pest management. Edited by Dyck VA, Hendrichs J, Robinson AS. Dordrecht, NL: Springer; 2005:427-451.

6. Parker A, Mehta K: Sterile insect technique: a model for dose optimization for improved sterile insect quality. Fla Entomol 2007, 90:88-95.

7. Handler AM: Prospects for using genetic transformation for improved SIT and new biocontrol methods. Genetica 2002, 116:137-49.

8. Schetelig MF, Wimmer EA: Insect Transgenesis and the Sterile Insect Technique. In Insect Biotechnology. Edited by Vilcinskas A. Dordrecht, NL: Springer Verlag; 2011:169_ 194.

9. Wimmer EA: Eco-friendly insect management. Nat Biotechnol2005, 23:432-433.

10. Alphey L, Benedict M, Bellini R, Clark GG, Dame DA, Service MW, Dobson SL: Sterileinsect methods for control of mosquito-borne diseases: an analysis. Vector Borne Zoonotic Dis. 2010, 10:295-311.

11. Simmons GS, McKemey AR, Morrison NI, O'Connell S, Tabashnik BE, Claus J, Fu G, Tang G, Sledge M, Walker AS, Phillips CE, Miller ED, Rose RI, Staten RT, Donnelly CA, Alphey L: Field performance of a genetically engineered strain of pink bollworm. PLoS One 2011, 6:e24110.

12. Harris AF, Nimmo D, McKemey AR, Kelly N, Scaife S, Donnelly CA, Beech C, Petrie WD, Alphey L: Field performance of engineered male mosquitoes. Nat Biotechnol 2011, 29:1034-7.

13. Lacroix R, McKemey AR, Raduan N, Kwee Wee L, Hong Ming W, Guat Ney T, Rahidah A A S, Salman S, Subramaniam S, Nordin O, Hanum A T N, Angamuthu C, Marlina Mansor S, Lees RS, Naish N, Scaife S, Gray P, Labbé G, Beech C, Nimmo D, Alphey L, Vasan SS, 
Han Lim L, Wasi A N, Murad S: Open field release of genetically engineered sterile male Aedes aegypti in Malaysia. PLoS One 2012, 7:e42771.

14. Mumford JD: Science, regulation, and precedent for genetically modified insects. PLoS Negl Trop Dis 2012, 6:e1504.

15. Carvalho DO, Nimmo D, Naish N, McKemey AR, Gray P, Wilke AB, Marrelli MT, Virginio JF, Alphey L, Capurro ML: Mass production of genetically modified Aedes aegypti for field releases in Brazil. $J$ Vis Exp 2014, 83:e3579.

16. Harris AF, McKemey AR, Nimmo D, Curtis Z, Black I, Morgan SA, Oviedo MN, Lacroix R, Naish N, Morrison NI, Collado A, Stevenson J, Scaife S, Dafa'alla T, Fu G, Phillips C, Miles A, Raduan N, Kelly N, Beech C, Donnelly CA, Petrie WD, Alphey L: Successful suppression of a field mosquito population by sustained release of engineered male mosquitoes. Nat Biotechnol2012, 30:828-30.

17. European Food Safety Authority Panel on Genetically Modified Organisms (GMO): Guidance on the environmental risk assessment of genetically modified animals. EFSA Journal 2013, 11:3200

18. Wilke $A B 1$, Marrelli $M T$ : Genetic control of mosquitoes: population suppression strategies. Rev Inst Med Trop Sao Paulo 2012, 54:287-92.

19. Reeves RG, Denton JA, Santucci F, Bryk J, Reed FA: Scientific standards and the regulation of genetically modified insects. PLoS Negl Trop Dis 2012, 6:e1502.

20. Phuc HK, Andreasen MH, Burton RS, Vass C, Epton MJ, Pape G, Fu G, Condon KC, Scaife S, Donnelly CA, Coleman PG, White-Cooper H, Alphey L: Late-acting dominant lethal genetic systems and mosquito control. BMC Biol2007, 5:11.

21. Benedict MQ, Robinson AS: The first releases of transgenic mosquitoes: an argument for the sterile insect technique. Trends Parasitol2003, 19:349-55.

22. Alphey $\mathrm{N}$, Bonsall MB, Alphey L: Modeling resistance to genetic control of insects. $J$ Theor Biol. 2011, 270:42-55.

23. MacIntosh SC: Managing the risk of insect resistance to transgenic insect control traits: practical approaches in local environments. Pest Management Science 2010, 66:100-106.

24. Robinson AS, Hendrichs J: Prospects for the future development and application of the sterile insect technique. In Sterile insect technique - principles and practice in areawide integrated pest management. Edited by Dyck VA, Hendrichs J, Robinson AS. Dordrecht, NL: Springer; 2005:727-760.

25. Gould F: Sustainability of transgenic insecticidal cultivars: integrating pest genetics and ecology. Annu Rev Entomol 1998, 43:701-26. 
26. Zhao JZ, Cao J, Li Y, Collins HL, Roush RT, Earle ED, Shelton AM: Transgenic plants expressing two Bacillus thuringiensis toxins delay insect resistance evolution. Nat Biotechnol2003, 21:1493-7.

27. Food and Agriculture Organization of the United Nations (FAO). Glossary of phytosanitary terms. Secretariat of the International Plant Protection Convention (IPPC). 2007, ISPM No 5.

28. Horn C and Wimmer EA: A transgene-based, embryo-specific lethality system for insect pest management. Nat Biotechnol 2003, 21:64-70.

29. Schetelig MF, Caceres C, Zacharopoulou A, Franz G and Wimmer EA: Conditional embryonic lethality to improve the sterile insect technique in Ceratitis capitata (Diptera: Tephritidae). BMC Biology 2009, 7:4.

30. Schetelig MF, Handler AM: Strategy for enhanced transgenic strain development for embryonic conditional lethality in Anastrepha suspensa. Proc Natl Acad Sci U S A. 2012, 109:9348-53.

31. Gong P, Epton MJ, Fu G, Scaife S, Hiscox A, Condon KC, Condon GC, Morrison NI, Kelly DW, Dafa'alla T, Coleman PG, Alphey L: A dominant lethal genetic system for autocidal control of the Mediterranean fruitfly. Nat Biotechnol 2005, 23:453-6.

32. Catteruccia F, Crisanti A, Wimmer EA: Transgenic technologies to induce sterility. Malar J. 2009, 8(Suppl 2):S7.

33. Nolan T, Papathanos P, Windbichler N, Magnusson K, Benton J, Catteruccia F, Crisanti A: Developing transgenic Anopheles mosquitoes for the sterile insect technique Genetica 2011, 139:33-9.

34. Windbichler N, Papathanos PA, Crisanti A: Targeting the $\mathbf{X}$ chromosome during spermatogenesis induces $\mathrm{Y}$ chromosome transmission ratio distortion and early dominant embryo lethality in Anopheles gambiae. PLoS Genet 2008, 4:e1000291.

35. Heinrich JC and Scott MJ: A repressible female-specific lethal genetic system for making transgenic insect strains suitable for a sterile-release program. Proc Natl Acad Sci USA 2000, 97, 8229-8232.

36. Thomas DD, Donnelly CA, Wood RJ and Alphey LS: Insect population control using a dominant, repressible, lethal genetic system. Science 2000, 287:2474-2476.

37. Fu G, Condon KC, Epton MJ, Gong P, Jin L, Condon GC, Morrison NI, Dafa'alla TH, Alphey L: Female-specific insect lethality engineered using alternative splicing. Nat Biotechnol2007, 25:353-7. 
38. Ant T, Koukidou M, Rempoulakis P, Gong HF, Economopoulos A, Vontas J, Alphey L: Control of the olive fruit fly using genetics-enhanced sterile insect technique. $B M C$ Biology 2012, 10:51.

39. Li F, Wantuch HA, Linger RJ, Belikoff EJ, Scott MJ: Transgenic sexing system for genetic control of the Australian sheep blow fly Lucilia cuprina. Insect Biochem Mol Biol 2014, 51:80-8.

40. Tan A, Fu G, Jin L, Guo Q, Li Z, Niu B, Meng Z, Morrison NI, Alphey L, Huang Y: Transgene-based, female-specific lethality system for genetic sexing of the silkworm, Bombyx mori. Proc Natl Acad Sci U S A 2013, 110:6766-70.

41. Schetelig MF, Handler AM: A transgenic embryonic sexing system for Anastrepha suspensa (Diptera: Tephritidae). Insect Biochem Mol Bio. 2012, 42:790-5

42. Ogaugwu CE, Schetelig MF, Wimmer EA: Transgenic sexing system for Ceratitis capitata (Diptera: Tephritidae) based on female-specific embryonic lethality. Insect Biochem Mol Biol2013, 43:1-8.

43. Koukidou M, Alphey L: Practical applications of insects' sexual development for pest control. Sex Dev 2014, 8:127-36.

44. Pane A, Salvemini M, Delli BP, Polito C and Saccone G: The transformer gene in Ceratitis capitata provides a genetic basis for selecting and remembering the sexual fate. Development 2002, 129:3715-3725.

45. Schetelig MF, Milano A, Saccone G, Handler AM: Male only progeny in Anastrepha suspensa by RNAi-induced sex reversion of chromosomal females. Insect Biochem Mol Biol2012, 42:51-7.

46. Gossen $\mathrm{M}$ and Bujard $\mathrm{H}$ : Tight control of gene expression in mammalian cells by tetracycline-responsive promoters. Proc Natl Acad Sci USA 1992, 89:5547-5551.

47. Bello B, Resendez-Perez D and Gehring WJ: Spatial and temporal targeting of gene expression in Drosophila by means of a tetracycline-dependent transactivator system. Development 1998, 125, 2193-2202.

48. Potter CJ, Tasic B, Russler EV, Liang L, Luo L: The Q system: a repressible binary system for transgene expression, lineage tracing, and mosaic analysis. Cell 2010, 141:536-48.

49. Potter CJ, Luo L: Using the Q system in Drosophila melanogaster. Nat Protoc 2011, 6:1105-20.

50. Wei X1, Potter CJ, Luo L, Shen K: Controlling gene expression with the Q repressible binary expression system in Caenorhabditis elegans. Nat Methods 2012, 9:391-5. 
51. Giles NH, Case ME, Baum J, Geever R, Huiet L, Patel V, Tyler B: Gene organization and regulation in the qa (quinic acid) gene cluster of Neurospora crassa. Microbiol Rev $1985,49: 338-58$.

52. Zulet A, Zabalza A, Royuela M. Phytotoxic and metabolic effects of exogenous quinate on Pisum sativum L. J Plant Growth Regul2013, 32:779-788.

53. Albertini MV, Carcouet E, Pailly O, Gambotti C, Luro F, Berti L: Changes in organic acids and sugars during early stages of development of acidic and acidless citrus fruit. J Agric Food Chem, 2006, 54:8335-9.

54. Giles NH, Geever RF, Asch DK, Avalos J, Case ME: The Wilhelmine E. Key 1989 invitational lecture. Organization and regulation of the qa (quinic acid) genes in Neurospora crassa and other fungi. J Hered 1991, 82:1-7.

55. Schetelig MF, Götschel F, Viktorinova I, Handler AM, Wimmer EA: Recombination technologies for enhanced transgene stability in bioengineered insects. Genetica 2011, 139:71-78.

56. Kosman D, Small S: Concentration-dependent patterning by an ectopic expression domain of the Drosophila gap gene knirps. Development 1997, 124:1343-54.

57. Wimmer EA, Cohen SM, Jäckle H, Desplan C: buttonhead does not contribute to a combinatorial code proposed for Drosophila head development. Development 1997, 124:1509-1517.

58. Carter JR, Fraser TS, Fraser MJ Jr: Examining the relative activity of several dicistrovirus intergenic internal ribosome entry site elements in uninfected insect and mammalian cell lines. J Gen Virol2008, 89:3150-5

59. Catteruccia F, Benton JP, Crisanti A: An Anopheles transgenic sexing strain for vector control. Nat Biotechnol2005, 23:1414-7.

60. Scolari F, Schetelig MF, Bertin S, Malacrida AR, Gasperi G and Wimmer EA: Fluorescent sperm marking to improve the fight against the pest insect Ceratitis capitata (Wiedemann; Diptera: Tephritidae). N Biotechnol 2008, 25:76-84.

61. Zimowska GJ, Nirmala $X$ and Handler AM: The beta2-tubulin gene from three tephritid fruit fly species and use of its promoter for sperm marking. Insect Biochem Mol Biol2009, 39:508-515.

62. Schetelig MF, Scolari F, Handler AM, Kittelmann S, Gasperi G, Wimmer EA: Site-specific recombination for the modification of transgenic strains of the Mediterranean fruit fly Ceratitis capitata. Proc Natl Acad Sci US A 2009, 106:18171-6. 
63. Galizi R, Doyle LA, Menichelli M, Bernardini F, Deredec A, Burt A, Stoddard BL, Windbichler N, Crisanti A: A synthetic sex ratio distortion system for the control of the human malaria mosquito. Nat Commun 2014, 5:3977.

64. Horvath P, Barrangou R: CRISPR/Cas, the immune system of bacteria and archaea. Science 2010, 327:167-70.

65. Wiedenheft B, Sternberg SH, Doudna JA: RNA-guided genetic silencing systems in bacteria and archaea. Nature 2012, 482:331-8.

66. Deltcheva E, Chylinski K, Sharma CM, Gonzales K, Chao Y, Pirzada ZA, Eckert MR, Vogel J, Charpentier E: CRISPR RNA maturation by trans-encoded small RNA and host factor RNase III. Nature 2011, 471:602-7.

67. Jinek M, Chylinski K, Fonfara I, Hauer M, Doudna JA, Charpentier E: A programmable dual-RNA-guided DNA endonuclease in adaptive bacterial immunity. Science2012, 337:816-21.

68. Fu Y, Sander JD, Reyon D, Cascio VM, Joung JK: Improving CRISPR-Cas nuclease specificity using truncated guide RNAs. Nat Biotechnol. 2014, 32:279-84.

69. Harrison MM, Jenkins BV, O'Connor-Giles KM, Wildonger J: A CRISPR view of development. Genes Dev. 2014, 28:1859-1872.

70. Esvelt KM, Smidler AL, Catteruccia F, Church GM: Concerning RNA-guided gene drives for the alteration of wild populations. Elife 2014, 17:e03401.

71. Kondo S, Ueda R: Highly improved gene targeting by germline-specific Cas9 expression in Drosophila. Genetics 2013, 195:715-21.

72. Port F, Chen HM, Lee T, Bullock SL: Optimized CRISPR/Cas tools for efficient germline and somatic genome engineering in Drosophila. Proc Natl Acad Sci U S A 2014, 111:E2967-76.

73. Renkawitz-Pohl R, Hempel L, Hollmann M, Schäfer MA: Spermatogenesis. In Comprehensive Molecular Insect Science, vol 1 Reproduction and Development. Edited by Gilbert LI, Iatrou K, Gill SS, Amsterdam:Elsevier Pergamon; 2005:157-177.

74. Sampson TR, Weiss DS: Exploiting CRISPR/Cas systems for biotechnology. Bioessays 2014, 36:34-8.

75. Xu T, Li Y, Van Nostrand JD, He Z, Zhou J: Cas9-based tools for targeted genome editing and transcriptional control. Appl Environ Microbiol. 2014, 80:1544-52. 


\section{Figures}
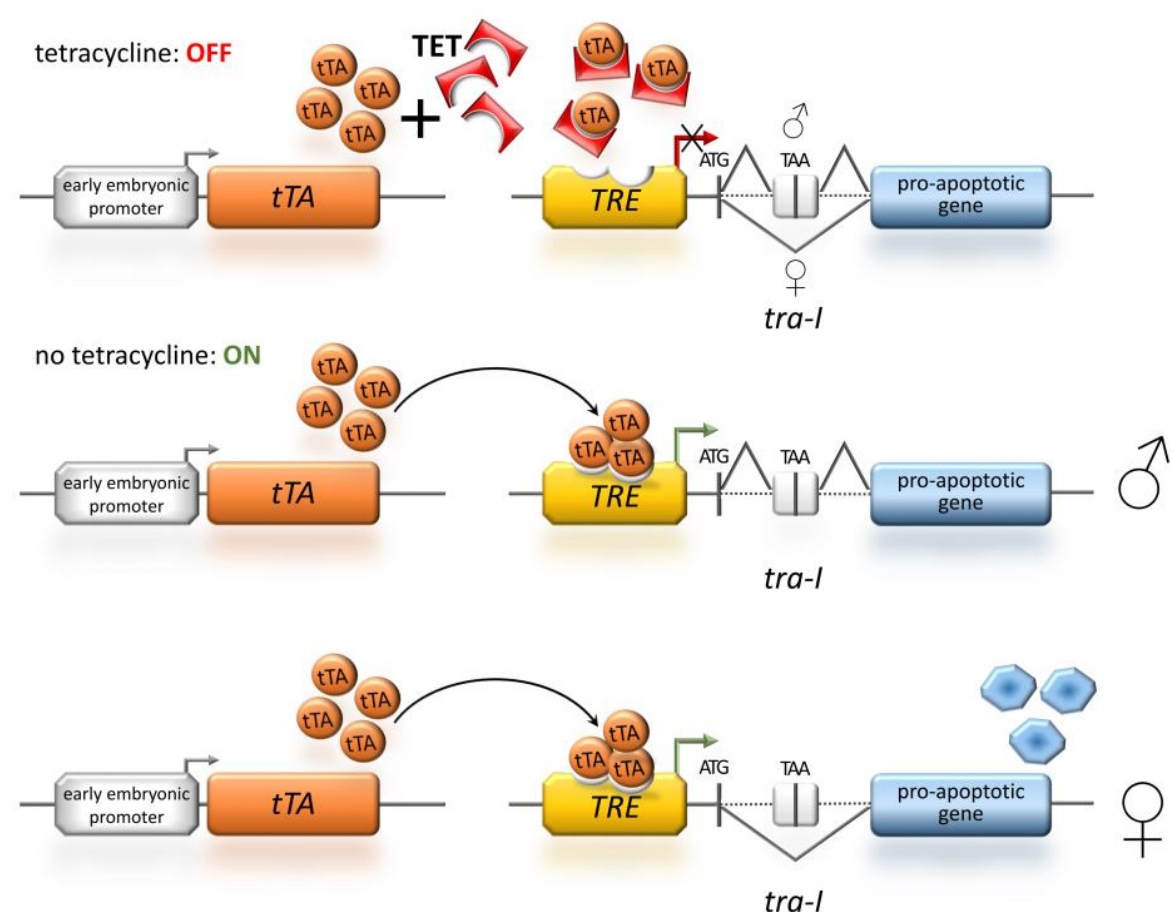

Figure - 1 Sexing using female-specific splicing under the control of the repressible tTA-system.

The depicted transgenic sexing system [41,42] uses a sex-specifically spliced intron and a hyperactive pro-apoptotic gene to generate female-specific lethality under the regulation of the tetracycline-controlled transactivator (tTA). To cause early embryonic lethality and thus avoidance of larval survival, the tTA is under the control of an early embryonic promoter. During rearing of such strains, addition of tetracycline (TET) to the food keeps the system in the OFF state, as tetracycline blocks the binding of tTA to its response element $(T R E)$. For the release generation, tetracycline is absent in the food and therefore the sexing system is $\mathrm{ON}$ : in males, the male specific splicing of the transformer intron (tra- $I$ - placed after the translation start codon (ATG) of the effector gene - includes a small exon containing a TAA stop codon between the start codon and the rest of the effector gene and therefore prevents the production of the functional pro-apoptotic effector protein allowing the males to survive; whereas in the females the female specific splicing of the tra- $I$ produces a functional effector and the embryonic cells are driven into apoptosis, which leads to female-specific embryonic lethality. 

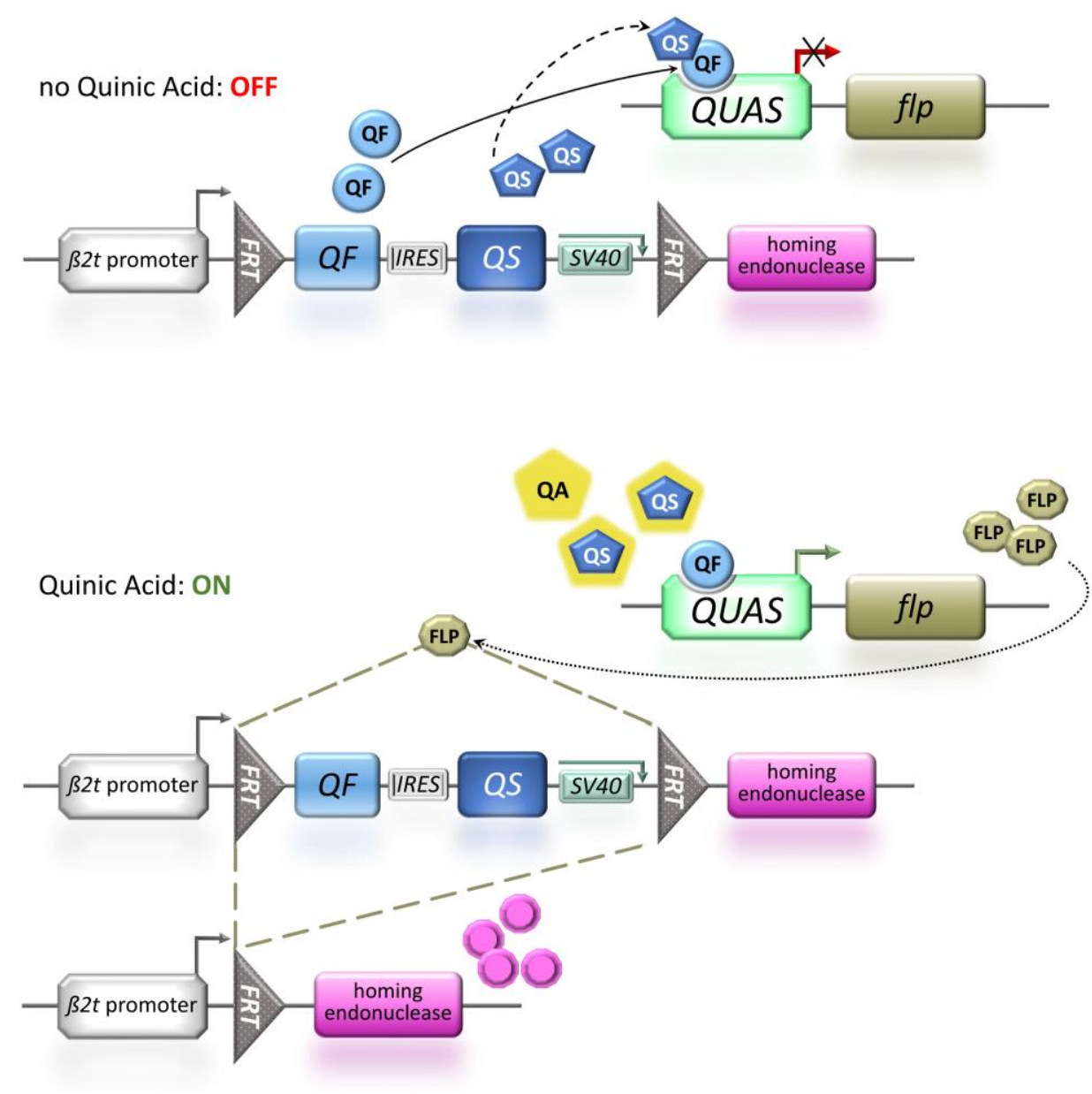

Figure 2 - Reproductive sterility using homing endonucleases controlled by the inducible Q-system in combination with site-specific recombination. The proposed reproductive sterility system is based on the inducible binary expression system Q [48], in which quinic acid (QA) acts as an inducer that hinders the repressor QS from complexing the transcriptional activator QF that can activate its target genes by binding to a $\mathrm{Q}$ upstream activation sequence (QUAS). To generate male reproductive sterility systems the spermatogenesis-specific promoter of the $\square 2$ tubulin ( $\square 2 t$ ) gene can be suitably used to affect either the sperm itself or the progeny sired by the sperm. The Q system can be combined with a recombinase mediated transcription regulation system to render the induction of an effector gene expression permanent and independent of the presence of the inducer QA. In this dual system, QF drives the expression of a site-specific recombinase (FLP) that can in turn remove a $f$-out cassette [57], which contains a transcriptional terminator (SV40) and is flanked by $f l p$ recombinant target sites $(F R T \mathrm{~s})$ in direct orientation. After the removal of the transcriptional terminator, the directed expression of an effector gene is mediated by the tissue-specific promoter 5' to the FRT. Since the Q system components are superfluous after the activation of the effector gene, they can also be placed into the $f$ p-out cassette. To make sure that both components of the Q system are translated in a bi-cistronic messenger RNA, they will be separated by an internal ribosome entry site (IRES). A homing endonuclease targeting the progeny genome can be employed as an effector that would kill the progeny but not the sperm itself [34]. During regular rearing this male reproductive sterility would be kept in an OFF state, as at the absence of QA the repressor QS will mask QF and block its activation potential. Only after the addition of QA to the food in the release generation, QS will be inactivated and QF thereby allowed to activate the expression of the $f l p$ recombinase (FLP), which in turn would remove the $\mathrm{Q}$ system regulators and at the same time activate the expression of the homing endonuclease that could block development of the next generation and thus cause male reproductive sterility. 

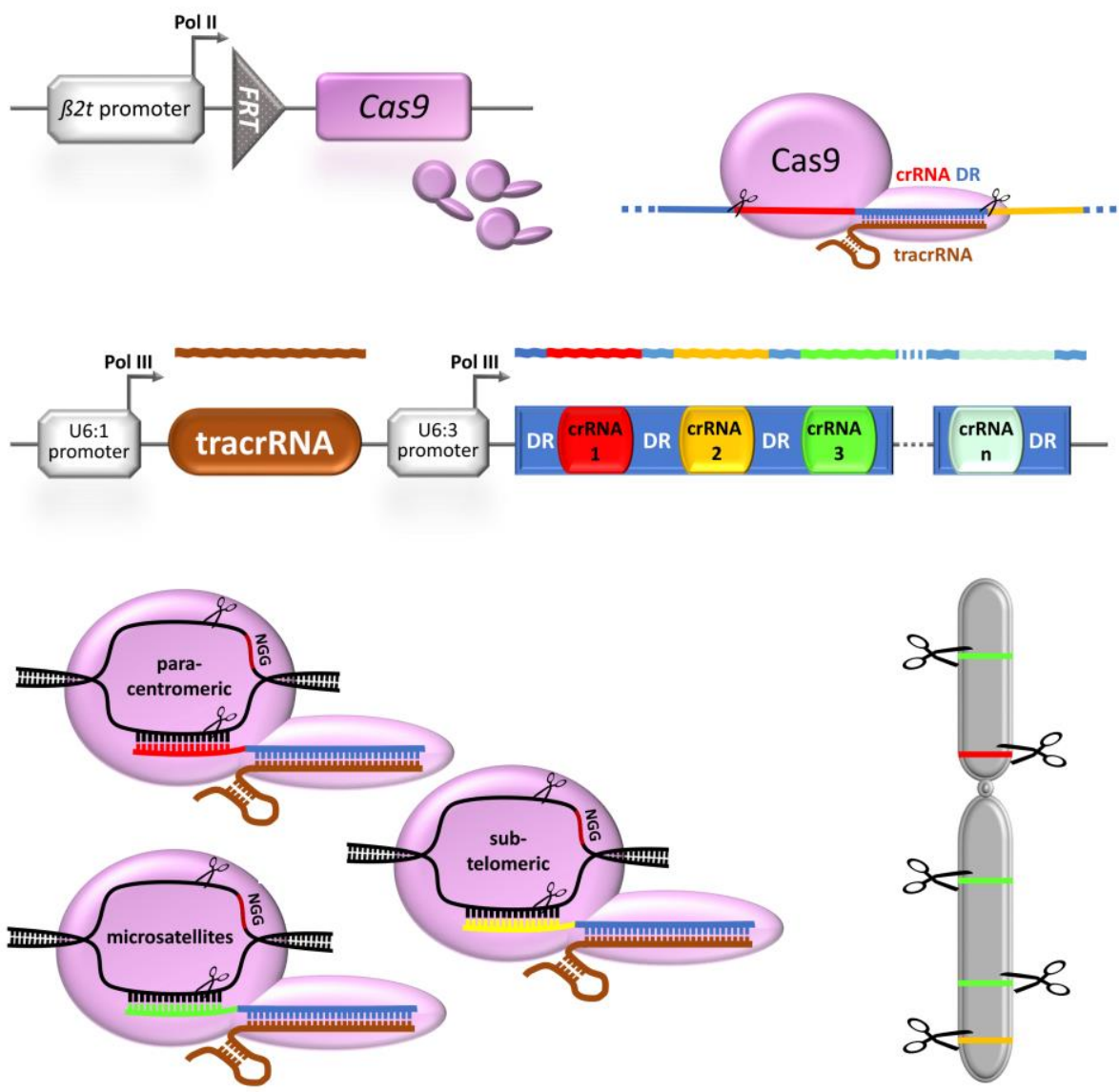

Figure 3- Redundant reproductive sterility based on the CRISPR/Cas9 system. The bacterial derived Cas9 endonuclease will be expressed under the control of the $\square 2$ tubulin $(\square 2 t)$ promoter. Cas 9 will be targeted to para-centromeric, sub-telomeric, and diverse macrosatellite sequences by guide RNAs, which are encoded by a CRISPR RNA (crRNA) array. This crRNA array as well as the trans-acting crRNA (tracrRNA) will be expressed under diverse RNA polymerase III promoters such as from the snRNA $U 6(\mathrm{U} 6: 1, \mathrm{U} 6: 3)$. In the crRNA array the diverse crRNAs are separated by direct repeat sequences (DR) derived from the Streptococcus pyogenes CRISPR. The expressed Cas9 is loaded with tracrRNA and subsequently binds the crRNA array based on complementarity between tracrRNA and the DR sequences, thereby randomly selecting one of the crRNAs as a guide to produce a functional CRISPR/Cas9 endonuclease targeting the respective genomic loci [75]. 
A Rearing: TET, no QA

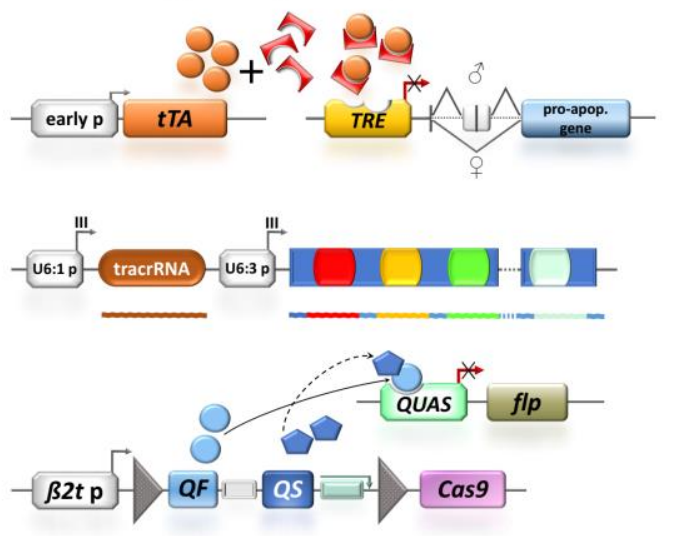

B Adult parents of release generation: no TET, no QA
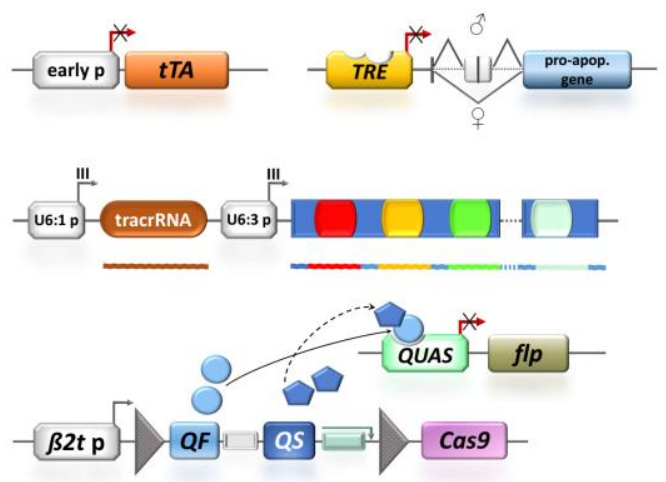

C Release generation: no TET, but QA
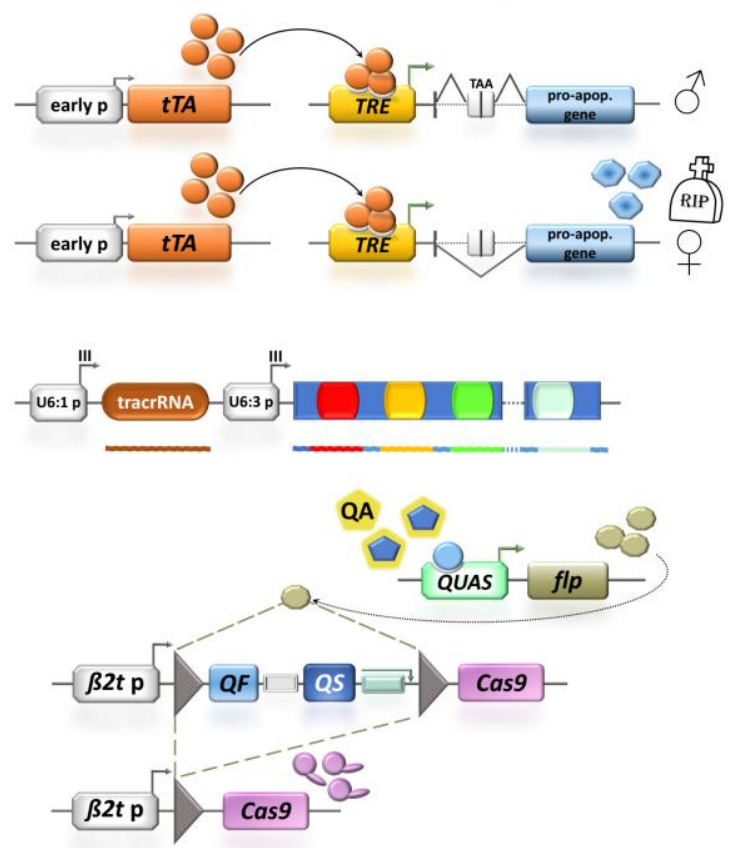

D Released males: no TET, no QA

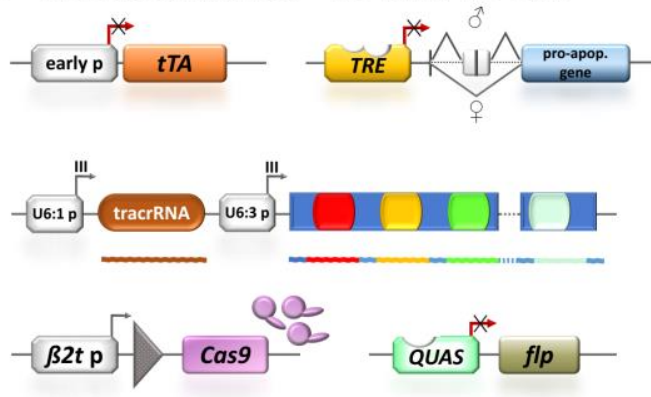

Figure 4 - Rearing scheme for combined female lethality and reproductive sterility systems

A Under regular rearing conditions, tetracycline (TET) is added to the food to repress the female lethality, quinic acid (QA) is not required for rearing. B The adult parents of the release generation will be changed to food without TET, still also without QA. This is necessary to avoid suppression of the early embryonic lethality in the next generation by maternally transferred TET to the oocyte. The female lethality system is still off, since the early embryonic promoter is not driving tTA at adult stages. C The release generation is then reared on food without TET but with added QA. Due to the lack of TET the female lethality system is switched on and the females die during early development. The QA leads to the activation of the $\mathrm{Q}$ system that leads to the expression of a site-specific recombinase, which in turn mediates the spermatogenesis-specific expression of the Cas 9 endonuclease by removing a recombination-site flanked spacer cassette. D The released males (no TET, no further QA) express high levels of the endonuclease Cas9 and multiple guide RNAs during spermatogenesis causing shredded chromosomes that will lead to lethal aneuploidy in the next generation. 


\subsection{Development of a CRISPR/Cas9-induced multifactorial reproductive sterility system based on sperm-specific chromosome shredding}

In this chapter, we present the first steps toward the development of a reproductive sterility system with proof of concept in D. melanogaster. The idea was initially described earlier (see chapter 3.6). We present a set of transgenic driver and Cas9 responder lines and their use to evaluate the suitability of the $\mathrm{E} / \mathrm{P}$ of the spermatogenesis-specific gene $\beta 2 t$, the tet-offsystem, and the basal promoters for the proposed system. Importantly, we present the identification of suitable CRISPR targets and the design of multiplexing constructs harboring two to three guide RNAs to increase the number of DSBs. We also present the first attempt to transfer the female-specific embryonic lethality system to the fruit pest D. suzukii and present possible solutions to failure regarding the generation of transgenic effector lines. All together this chapter presents a new strategy for the development of the SIT. If this system works as predicted, it can be combined with sexing and sperm-marking systems to have an all in one SIT package to fight agricultural pests and diseases vectors.

Hassan M. M. Ahmed, Fabienne Heese, \& Ernst A. Wimmer

Authors contributions to practical work:

Hassan M. M. Ahmed: All experiments besides the ones performed by FH. This also includes target identification and genetic construct design.

Fabienne Heese: Generation of transgenic lines.

Status: $\quad$ Work in progress 


\section{Development of a CRISPR/Cas9-induced multifactorial reproductive sterility system based on sperm-specific chromosome shredding}

Hassan M. M. Ahmed ${ }^{1,2}$, Fabienne Heese ${ }^{1}$, \& Ernst A. Wimmer ${ }^{{ }^{*}}$

${ }^{1}$ Department of Developmental Biology, Johann-Friedrich-Blumenbach-Institute of Zoology and Anthropology, Göttingen Center for Molecular Biosciences, Georg-August-University Göttingen, 37077 Göttingen, Germany.

${ }^{2}$ Department of Crop Protection, Faculty of Agriculture-University of Khartoum P.O. Box 32, Postal Code 13314

Khartoum North, Khartoum, Sudan

*Corresponding Author: E. A. Wimmer. Tel. +495513928666

E-Mail address: ewimmer@gwdg.de (E.A. Wimmer).

\section{Background}

Resistance development is a major concern when developing a new system for pest management, especially because insects have short generation time and high fecundity, which makes emergence of resistance against the control system very likely (1). We have proposed the use of CRISPR/Cas9 to develop a new reproductive sterility system that is characterized by redundancy, which means the source of sterility is not based on one particular gene locus and should therefore overcome the possibility of resistance development (2). Three prerequisites need to be fulfilled to develop such a system: (1) abundant CRISPR targets, (2) enhancer/promoter that is spermatogenesis specific, and (3) a suitable binary expression system to facilitate conditional activation of the reproductive sterility system. Targeting Cas9 to multiple genomic loci such as para-centromeric, sub-telomeric, and microsatellites to induce simultaneously many double strand breaks (DSBs) will mimic the action of ionizing radiation specifically during spermatogenesis and does not damage necessary genes (2). This will lead to production of competent males that are better suited to compete with the wild type males. Multiplexing of CRISPR target sites using the transfer RNA ( $t R N A)$ processing system was demonstrated in plants and in Drosophila (3-5). CRISPR/Cas9 driven by the enhancer/promoter of spermatogenesis-specific genes has proven to be a suitable effector to induce chromosome shredding, which was demonstrated in the mosquito Anopheles gambiae by targeting it to the rDNA on the $\mathrm{X}$ chromosome to allow for production of only $\mathrm{Y}$ chromosome bearing sperm (6). In the same study, they used the enhancer/promoter of the gene $\beta 2 t$ to confine the expression of Cas 9 to spermatogenesis. In fact, $\beta 2 t \mathrm{E} / \mathrm{P}$ was successfully used in previous study to direct the expression of the $\mathrm{X}$-shredder homing endonuclease gene (HEG) I-PpoI to spermatogenesis and generated a sex ratio distortion system $(6,7)$. The enhancer/ promoter of the Drosophila $\beta 2 t$ gene is a great candidate to drive strong expression of effector molecules during spermatogenesis in a conditional manner. Three binary expression systems (tet-on, tet-off, and the Q-system) (8-12) are of relevance and can be used to control and confer conditionality to the system. In fact, to realize the ultimate goal of this 
study by combining the reproductive sterility and the sexing systems, two independent binary system are needed (2). Other interesting genes that are spermatogenesis specific and are also regulated at the level of translation by translation arrest are Don juan $(d j)$ and Don juan like (dj) in D. melanogaster (13). Interestingly the sister genes are next to each other and both are regulated post-transcriptionally by a translation suppression element in their 5'UTR. This sequence element leads to translation delay of the mRNA until post meiotic stages, when the genes are needed and translated. In this study we aim to develop a redundant male sterility system by targeting Cas9 to transposable elements during spermiogenesis, to avoid interfering with production of sperm and the targeting of somatic chromosomes. To this end, we will test the suitability of the tet-off system to control gene expression during spermatogenesis. Furthermore, we will exploit the translation delay element of the Dm dj gene to delay the translation of Cas9 to post-meiotic stages of spermatogenesis.

To eventually be able to transfer these systems to the invasive pest $D$. suzukii and to combine the reproductive sterility system with the well establish transgenic female-specific embryonic lethality system (FSEL), we have also taken the first steps in establishing FSEL in D. suzukii.

\section{Results}

\section{Identification of CRISPR targets for chromosome shredding}

To be able to use the CRISPR/Cas9 system to induce reproductive sterility equivalent to the action of ionizing radiation, we need to induce as many double strands breaks as possible. To achieve that we searched for euchromatin transposable elements in the genome of $D$. melanogaster. We based our search on Kaminker et al. (14), since heterochromatin might not be accessible for Cas9. Based on their overall representation in the genome and the distribution across the chromosomes, we have chosen the three transposable element families, roo, jockey, and 1360, representing three classes: long terminal repeat LTR, LINE-like, and inverted terminal repeat ITR. Roo is represented by 146 elements distributed on all chromosomes except chromosome 4. jockey is identified 69 times with distribution on all chromosomes including two times on chromosome 4 . The family 1360 appears 105 times with distribution on all chromosomes including 30 times on chromosome 4.

Three guide RNAs for each element were designed based on the latest $D$. melanogaster genome release, $\mathrm{r}-6$ (15). Each gRNA is shown in Table S2 with the number of exact matches in the genome of $D$. melanogaster.

\section{Design of constructs and generation of transgenic lines}

To drive conditional and testes specific expression of Cas 9 and $g R N A s$ targeting transposable elements, we used the tet-off binary expression system and $E / P$ of the spermatogenesis specific genes $D m-\beta 2 t$ and $D m-d j$. We generated driver construct HMMA334 by fusion of $D m-\beta 2 t$ $E / P$ upstream of the heterologous transcription factor tetracycline transactivator $(t T A)$. The construct was use to generate 24 independent transgenic lines. We also used the vector HMMA324 (chapter 3.5) to conditionally and simultaneously express two gRNAs targeting 
either of the three transposable elements roo, jockey, and 1360. In addition, we used vector HMMA349 (chapter 3.5) to generate $g R N A$-driver constructs HMMA446, HMMA447, and HMMA448 to constitutively and simultaneously express three $g R N A s$ targeting the same TEs, and construct HMMA449 to express one target for each of the three TEs.

Unlike in the case of using Cas 9 to target paternal effect genes, which do not interfere with male fitness or spermatogenesis, we aim to delay the effect in the proposed CRISPR/Cas9 chromosome shredding system to post-meiosis stages of spermatogenesis to avoid interfering with spermatogenesis. To achieve that we utilized the translation suppression element from the 5' UTR of the Dm-dj gene or the 5'UTR of the Dm- $\beta 2 t$ gene. This sequence, when fused upstream of a gene, is supposed to delay the translation of that gene to the post-meiotic stage (Fig. 1D). We generated three different Cas 9 responder constructs HMMA365, HMMA366, and HMMA203 by fusion of the insect codon optimized Cas 9 coding sequence (with $\mathrm{N}$ and $\mathrm{C}$-terminal nuclear localization signal and an $\mathrm{N}$-terminal Flag tag) downstream of the TRE with the basal promoter of either the P-element or Dm-dj and both have dj TSE just upstream of the Cas 9 translation start codon. The third construct HMMA203 has the $P_{-}$ element basal promoter and the 5' UTR from $D$. suzukii $\beta 2 t$ gene. The responder constructs HMMA203 was used to generate 10 independent transgenic lines, HMMA365 (29 independent lines), and HMMA366 (12 independent lines).

\section{Testes specific expression of Cas9}

To check whether tTA can drive testes specific expression of Cas9 under the control of the TRE with the P-element or $d j$-basal promoter, we crossed driver lines 334_F1F4 and 334_F48M1 separately to responder lines 365_M7_F1, 366_M7F1, and 204_M1F1. We then performed RT-PCR on total RNA isolated from the testes of flies carrying both constructs (Fig. 2C). The results show that Cas9 is expressed from responders with $P$-element basal promoter with (365_M7_F1) and without the TSE (204_M1F1) and weak expression was detected from responder with $d j$-basal promoter (366_M7F1), however, this is most likely specific to this particular line and not a construct problem. Worth noting is detection of Cas 9 expression in the negative control (Cas9 responder not crossed to driver). However, this can be due to high sensitivity of the assay and or leakiness of the basal promoter, which needs to be evaluated.

\section{Discussion}

The SIT offers a clean pest control approach since it is species-specific and does not harm natural enemies and pollinators (16). Sterility in the released males is induced by ionizing radiation, which is very effective in this regard and resistance development against it is unlikely due to random chromosome breakdown causing reproductive sterility (17). However, the males' fitness is also affected $(17,18)$ and therefore the area targeted for control with SIT must be flooded with a large amount of such sterile males to compete with the wild type males in numbers rather than in individual fitness. To overcome the fitness cost due to irradiation, we 
proposed to develop a reproductive sterility system similar to ionizing radiation with regard to redundancy, and therefore a reduced chance of resistance development, but superior to it in the sense that the fitness of the sterile males is not compromised (2). The system relies on the use of the CRISPR/Cas9 system to induce many double strand breaks in the sperm chromosomes during spermatogenesis, not affecting the males themselves or the process of sperm production. Therefore, males should transfer sperm with multiple chromosomal aberrations due to incorrect repair of the DSBs (2). Such sperm should be uncapable of forming a functional zygote and as a result embryonic development should not start. To achieve that, we searched for $D$. melanogaster transposable elements (TEs) and we have chosen three families, roo, jockey, and 1360 for proof of concept in D. melanogaster. Three gRNAs targeting each TE were designed and used to generate gRNA-driver constructs using the same multiplexing strategies described in chapter 3.5. The total number of genomic targets for construct HMMA448 with three gRNAs against 1360 are 874, for construct HMMA447 targeting jockey are 212, and for HMMA446 targeting roo are 379. The combination of 1360_gRNA2, roo_gRNA26, and jockey_gRNA10 together in construct HMMA449 should target and induce 661 DSBs. These overwhelming numbers of target sites combined with abundant expression of Cas9 and gRNAs should with lead to many chromosomal aberrations culminating in embryonic lethality and thus provide a suitable way to cause reproductive sterility. This, however, should be induced post meiotically to avoid interfering with meiosis and spermatogenesis. To induce such chromosomal abnormalities at the post meiotic stage of spermatogenesis, fine coordination of transcription and translation of the effector molecule Cas9 is required. The tissue specificity and conditionality are supposed to be achieved by the use of the $\mathrm{P} / \mathrm{E}$ of the spermatogenesis-specific gene $D m-\beta 2 t$ and the tetoff binary expression system. It is important that enough Cas 9 transcript is produced during the mitotic amplification divisions before transcription shutdown in the maturing primary spermatocytes. However, the translation of these transcripts should not start until after meiosis has completed and spermiogenesis started. To arrange for that, we fused the translation repression element of the $D$. melanogaster djgene (19) in front of the Cas 9 coding sequence. This shouldn't interfere with Cas 9 transcription but the mRNA should undergo translational arrest until the end of meiosis and start of spermiogenesis (Fig. 1D).

Crossing spermatogenesis specific driver lines to Cas9 responder lines should allow investigation of whether the $\beta 2 t E / P$ is suitable to drive the $t T A$ and whether the later can activate $\operatorname{Cas} 9$ expression. Our attempt to test the expression of $\operatorname{Cas} 9$ during spermatogenesis by means of RT-PCR was not conclusive since we also detected Cas 9 transcripts in the negative, driver-less control (Fig. 2C). It is likely that the RT-PCR is very sensitive and can detect low basal expression from the minimal promoters fused to the $t T A$ responsive element $T R E$ (Fig. 2C). In situ hybridization and antibody staining against Cas $9 \mathrm{mRNA}$ and protein, respectively, will be more informative and can also give information about the stage of 
spermatogenesis at which cas 9 is transcribed and whether the translational repression element is functional to delay translation of Cas $9 \mathrm{mRNA}$ to post meiotic stages. The basal expression due to leakiness of promoters used might be harmful for the fertility of the males during rearing. Thus, the use of insulators might reduce the likeliness of expression due to position effects caused by nearby enhancers (20-22). However, identification and evaluation of a tight basal promoter, preferably from genes involved in spermatogenesis might provide a better solution.

To prepare all the components necessary for a fully equipped transgenic SIT approach against D. suzukii, we started to transfer the female-specific embryonic lethality system as a sexing system to remove females during early embryonic development and thus produce male-only progenies (23-25). The embryonic driver line 06_F5M2 described in Ahmed et al., (26) as well as the embryonic driver line 319_F11F1, which are the first components. Our attempt to generate transgenic effector lines expressing hid ${ }^{A l a}$ and having the sex-specifically spliced intron of the gene transformer either from Ceratitis capitata HMMA423 with no insulator or D. suzukii HMMA108 was not successful. The constructs HMMA108 have the effector gene flanked by gypsy insulators, which, however, might not provide enough protection. In $D$. melanogaster, using similar constructs without insulators didn't result in transgenic lines since basal expression of the effector gene from the minimal promoter was considered high enough to induce cell death (27). Only when the HS4 insulator was used, it was possible to generate functional strains (27). In fact, using construct HMMA322 with HS4 insulators, we managed to generate one transgenic line but could not establish a strain, since the transgenic $F_{1}$ fly was lost, before we could cross it. Another possible explanation can be that the P-element basal promoter is too leaky in D. suzukii. With this regard, testing different basal promoters, preferentially endogenous ones, for their suitability is necessary.

Development of a CRISPR/Cas9-induced reproductive sterility system in D. melanogaster should provide a first proof of concept and pave the way for the transfer to the destructive fruit pest $D$. suzukii. This system, if successful, would represent a milestone in the fight against the cherry vinegar fly. Ultimately, combining this system with the female specific embryonic lethality system as described in (2) and the sperm-marking system described in Ahmed et al., (26) results in an all in one system to launch an SIT campaign against D. suzukii. This system can as well be combined with the aforementioned reproductive sterility system based on targeting PEEL genes to have less chances of resistance against the SIT. 


\section{References}

1. KaramiNejadRanjbar M, Eckermann KN, Ahmed HMM, Sánchez C. HM, Dippel S, Marshall $\mathrm{JM}$, et al. Consequences of resistance evolution in a Cas9-based sex conversion-suppression gene drive for insect pest management. Proc Natl Acad Sci USA. 2018 Jun 12;115(24):6189-94.

2. Eckermann KN, Dippel S, KaramiNejadRanjbar M, Ahmed HM, Curril IM, Wimmer EA. Perspective on the combined use of an independent transgenic sexing and a multifactorial reproductive sterility system to avoid resistance development against transgenic Sterile Insect Technique approaches. BMC Genet. 2014;15(Suppl 2):S17.

3. Xie K, Minkenberg B, Yang Y. Boosting CRISPR/Cas9 multiplex editing capability with the endogenous tRNA-processing system. PNAS. 2015 Mar 17;112(11):3570-5.

4. Port F, Bullock SL. Augmenting CRISPR applications in Drosophila with tRNA-flanked sgRNAs. Nat Methods. 2016 Oct;13(10):852-4.

5. Qi W, Zhu T, Tian Z, Li C, Zhang W, Song R. High-efficiency CRISPR/Cas9 multiplex gene editing using the glycine tRNA-processing system-based strategy in maize. BMC Biotechnol. 2016 Dec;16(1):58.

6. Galizi R, Hammond A, Kyrou K, Taxiarchi C, Bernardini F, O'Loughlin SM, et al. A CRISPR-Cas9 sex-ratio distortion system for genetic control. Sci Rep. 2016 Nov;6(1):31139.

7. Windbichler N, Papathanos PA, Crisanti A. Targeting the $\mathrm{X}$ Chromosome during Spermatogenesis Induces Y Chromosome Transmission Ratio Distortion and Early Dominant Embryo Lethality in Anopheles gambiae. PLOS Genetics. 2008 Dec 5;4(12):e1000291.

8. Giles NH, Geever RF, Asch DK, Avalos J, Case ME. Organization and Regulation of the Qa (Quinic Acid) Genes in Neurospora crassa and Other Fungi. J Hered. 1991 Jan 1;82(1):1-7.

9. Wei X, Potter CJ, Luo L, Shen K. Controlling gene expression with the Q repressible binary expression system in Caenorhabditis elegans. Nat Methods. 2012 Apr;9(4):391-5.

10. Zhou X, Vink M, Klaver B, Berkhout B, Das AT. Optimization of the Tet-On system for regulated gene expression through viral evolution. Gene Ther. 2006 Oct;13(19):1382-90.

11. Gossen M, Bujard H. Tight control of gene expression in mammalian cells by tetracyclineresponsive promoters. Proceedings of the National Academy of Sciences. 1992 Jun 15;89(12):5547-51.

12. Urlinger S, Baron U, Thellmann M, Hasan MT, Bujard H, Hillen W. Exploring the sequence space for tetracycline-dependent transcriptional activators: Novel mutations yield expanded range and sensitivity. Proceedings of the National Academy of Sciences. 2000 Jul 5;97(14):79638.

13. Hempel LU, Rathke C, Raja SJ, Renkawitz-Pohl R. In Drosophila, don juan and don juan like encode proteins of the spermatid nucleus and the flagellum and both are regulated at the 
transcriptional level by the TAFII80 cannonball while translational repression is achieved by distinct elements. Developmental Dynamics. 2006;235(4):1053-64.

14. Kaminker JS, Bergman CM, Kronmiller B, Carlson J, Svirskas R, Patel S, et al. The transposable elements of the Drosophila melanogaster euchromatin: a genomics perspective. Genome Biology. 2002 Dec 23;3(12): research0084.1.

15. Hoskins RA, Carlson JW, Wan KH, Park S, Mendez I, Galle SE, et al. The Release 6 reference sequence of the Drosophila melanogaster genome. Genome Res. 2015 Mar 1;25(3):445-58.

16. Knipling EF. Possibilities of Insect Control or Eradication Through the Use of Sexually Sterile Males1. Journal of Economic Entomology. 1955 Aug 1;48(4):459-62.

17. Bakri A, Mehta K, Lance DR. Sterilizing Insects with Ionizing Radiation. In: Dyck VA, Hendrichs J, Robinson AS, editors. Sterile Insect Technique: Principles and Practice in AreaWide Integrated Pest Management [Internet]. Dordrecht: Springer Netherlands; 2005 [cited 2019 Nov 17]. p. 233-68. Available from: https://doi.org/10.1007/1-4020-4051-2_9

18. Barry JD, McInnis DO, Gates D, Morse JG. Effects of irradiation on Mediterranean fruit flies (Diptera: Tephritidae): emergence, survivorship, lure attraction, and mating competition. J Econ Entomol. 2003 Jun;96(3):615-22.

19. Blümer N, Schreiter K, Hempel L, Santel A, Hollmann M, Schäfer MA, et al. A new translational repression element and unusual transcriptional control regulate expression of don juan during Drosophila spermatogenesis. Mechanisms of Development. 2002 Jan 1;110(1):97112.

20. Chung JH, Bell AC, Felsenfeld G. Characterization of the chicken $\beta$-globin insulator. PNAS 1997 Jan $21 ; 94(2): 575-80$

21. Kang HJ. The functional role of the Drosophila gypsy insulator in the regulation of gene expression. Doctoral Dissertations [Internet]. 2010 May 1; Available from: https://trace.tennessee.edu/utk_graddiss/710

22. Sarkar A, Atapattu A, Belikoff EJ, Heinrich JC, Li X, Horn C, et al. Insulated piggyBac vectors for insect transgenesis. BMC Biotechnol. 2006 Jun 16; 6:27.

23. Ogaugwu CE, Schetelig MF, Wimmer EA. Transgenic sexing system for Ceratitis capitata (Diptera: Tephritidae) based on female-specific embryonic lethality. Insect Biochemistry and Molecular Biology. 2013 Jan;43(1):1-8.

24. Schetelig MF, Handler AM. A transgenic embryonic sexing system for Anastrepha suspensa (Diptera: Tephritidae). Insect Biochemistry and Molecular Biology. 2012 Oct;42(10):790-5.

25. Yan Y, Scott MJ. A transgenic embryonic sexing system for the Australian sheep blow fly Lucilia cuprina. Sci Rep. 2015 Dec;5(1):16090.

26. Ahmed HMM, Hildebrand L, Wimmer EA. Improvement and Use of CRISPR/Cas9 to Engineer a Sperm-marking Strain for the Invasive Fruit Pest Drosophila suzukii. 2019 Aug 14 
[cited 2019 Nov 2]; Available from: https://www.researchsquare.com/article/804bbff3-022844d5-8e09-3acb0d8e408e/v1

27. Horn C, Wimmer EA. A transgene-based, embryo-specific lethality system for insect pest management. Nat Biotechnol. 2003 Jan;21(1):64-70. 
Figures:

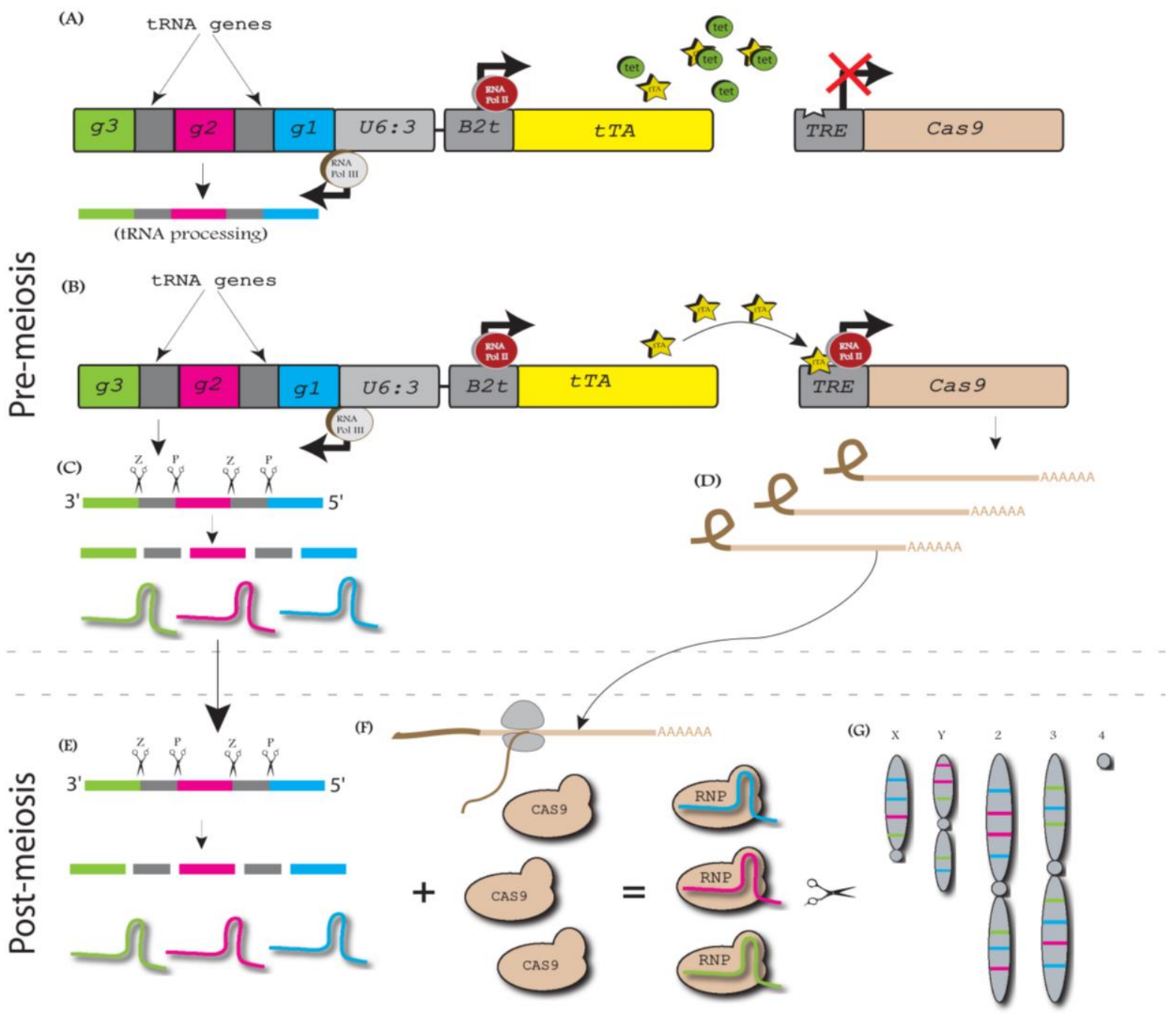

Figure. 1. Schematic illustration of the CRISPR/Cas9-based chromosome shredding system. (A) the system consists of a dual gRNA-driver construct and a Cas 9 responder construct controlled by the tet-off binary expression system. In the first part, heterologous transcription factor tTA is expressed during spermatogenesis under the control of $E / P$ of $D$. melanogaster spermatogenesis-specific gene $\beta 2 t$ and the guide $R N A s(g R N A \mathrm{~s})$ are constitutively transcribed by RNA polIII from the promoter of $D$. melanogaster small nuclear $R N A$ gene $U 6: 3$. In the presence of tetracycline in the fly food, the tTA is bound by tet and can not bind to the $t T A$ responsive element $(T R E)$ and therefore Cas 9 is not produced and the system is off. This should be the situation during establishment, maintenance and mass-production of the strain. (B) In the absence of tet in the fly food, tTA is free to bind the TRE and drive the transcription of Cas 9 during pre-meiosis stages of spermatogenesis. (D) However, Cas9 mRNA should undergo translational arrest until post meiosis due to fusion of the translational repression element from $D m$ dj gene upstream of Cas 9 coding sequence. (C) Three gRNAs are interspaced by tRNA genes which facilitate their processing into individual $g R N A$ by the $t R N A$ processing endonucleases $\mathrm{P}$ and $\mathrm{Z}$. (E) during post meiosis $g R N A s$ should still be available. (F) Cas 9 arrest should be relieved by factors available during spermiogenesis and will then be translated into Cas9 protein and complex with the $g R N A s$ forming the ribonucleoprotein complex. (G) Cas9 loaded with gRNAs should target the chromosomes and induce multiple double strand breaks. This provide many sticky ends that leads to chromosome rearrangements among other aberration leading at the end to embryonic lethality. 


\section{(A)}

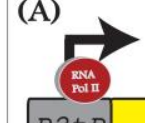

$B 2 t P$

tTA $\quad$ SV40 耑圆

(B)

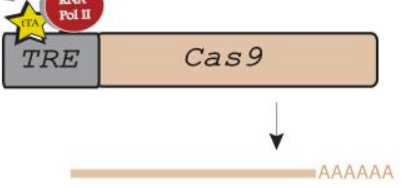

(C)

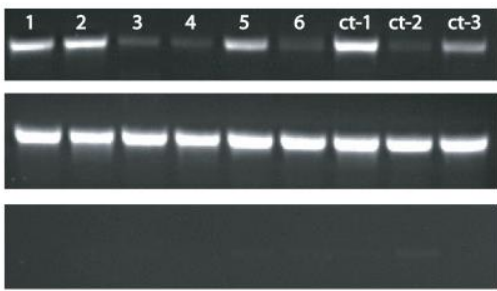

Figure. 2. Cas9 expression during spermatogenesis (A) the heterologous transcription factor tetracycline controlled transactivator $\mathrm{tTA}$ is driven by the $\mathrm{E} / \mathrm{P}$ of $D$. melanogaster spermatogenesis specific gene $\beta 2 t$ gene $(\mathbf{B})$ shows the expression of Cas 9 under the control of TRE directed by spermatogenesis-specific expression of the $t T A$. (C) the upper panel of the gel picture shows that Cas9 is expressed in the testes of double heterozygous $D$. melanogaster strain harboring the two components shown in $(\mathbf{A})$ and $(\mathbf{B})$. each of the chosen three responder lines was crossed individually to two gRNA-driver line. Each two consecutive numbers represent one responder line crossed to driver lines 372_M14_M1 and 372_F26_F1 respectively. The last three lanes are RT-PCR negative control using the respective responder line not crossed to any driver. Ct- 1 controls for 1 and 2, ct-2 controls for 3 and 4, and ct3 controls for 5 and 6 . Ideally the negative controls should not express Cas9. However, basal promoters can drive expression at a basal level depends on the position of integration. The middle lane is the positive control for the RT-PCR procedure using primer pair to amplify from the same cDNA pool the 3'UTR of the spermatogenesis-specific $\beta 2 t$ gene. All of the lanes gave strong bands which reflect the reliability of the tests, especially when looking at the result on the light of the lower lane which represent the RT-less PCR following the same procedure and the same amount of the initial RNA but without addition of the reverse transcriptase. Cas 9 expression in the negative non-crossed control in the upper doesn't not necessarily means that the responder lines are not functional due to leakiness, but the PCR is highly sensitive and can detect low levels of expression from very few molecules. 


\section{Materials and Methods}

\section{Drosophila suzukii strains}

All fly experiments were performed in our well-equipped safety level one (S1) laboratory, which is certified for generating and using genetically modified insects.

Wild type D. melanogaster Oregon R strain as well as the transgenic flies generated during this study were reared on standard Drosophila food and kept at $25^{\circ} \mathrm{C}$ throughout this study.

\section{Nucleic acid isolation}

Genomic DNA was isolated from adult males and females using NucleoSpin ${ }^{\circledR}$ DNA Insect (Macherey-Nagel) according to the manufacturer instructions. Total RNA was isolated from testes of 4-5 days old males using ZR Tissue \& Insect RNA MicroPrep (Zymo Research Europe, 79110 Freiburg) according to manufacturer instructions.

PCR amplifications during this study were performed using Phusion DNA polymerase and Phusion-HF buffer (New England Biolabs GmbH, D-65926 Frankfurt am Main). Plasmid isolation and PCR purification were performed using NucleoSpin ${ }^{\circledR}$ Plasmid and NucleoSpin ${ }^{\circledR}$ Gel and PCR Clean-up kits (Macherey-Nagel GmbH \& Co., 52355 Dueren, Germany), respectively. NucleoSpin ${ }^{\circledR}$ Plasmid Transfection-grade (Macherey-Nagel) or QIAGEN Plasmid Plus Midi Kit (QIAGEN GmbH, 40724 Hilden, Germany) were used to prepare plasmids for germline transformation.

\section{Identification of abundant CRISPR targets}

We have chosen three families of $D$. melanogaster euchromatin transposable elements, roo, jockey and 1360 on the ground that they have higher chance to be accessed by Cas9. We retrieve the sequence from https://www.fruitfly.org/p_disrupt/TE.html natural transposable elements project. Natural transposable elements dataset Maintained by Michael Ashburner was downloaded and the sequence of the chosen TE was manually retrieved and examined using program version 10.2.6 (Auckland, 1010, New Zealand). The sequences were then blasted in https://flybase.org/ against $D$. melanogaster genome r-6 and the part of the sequence that showed the most coverage possibly over all the chromosomes was used to search for potential CRISPR targets. To identify abundant targets the online tool maintained by Wisconsin university was used. About $300 \mathrm{bp}$ of the chosen sequenced was use to find targets with the following parameters: target length $20 \mathrm{bp}$, all CRISPR targets, PAM sequence NGG. The obtained targets were evaluated based on the latest $D$. melanogaster release $\mathrm{r}-6$ and the targets with the most abundant representation but also exact matches were chosen.

\section{Generation of constructs}

To generate the spermatogenesis-specific driver construct HMM334, first the 700bp enhancer/promoter region of $D m-\beta 2 t$ was PCR amplified from gDNA of $D$. melanogaster 
wild type strain OreR using primers pair HM\#351/HM\#352 and program $\left(98^{\circ} \mathrm{C} 3^{\prime} 57^{\circ} \mathrm{C} 30^{\prime \prime}\right.$ $\left.73^{\circ} \mathrm{C} 30^{\prime \prime}\right) 5 \mathrm{X}$ followed by $35 \mathrm{X}$ of $\left(98^{\circ} \mathrm{C} 30^{\prime \prime} 63^{\circ} \mathrm{C} 30^{\prime \prime} 72^{\circ} \mathrm{C} 30^{\prime \prime}\right)$ and final elongation of 7 min at $72{ }^{\circ} \mathrm{C}$, digested by $N c o I / X b a I$ and cloned into msf\#1215 $N c o I / X b a I$ sites giving rise to HMMA175. The $450 \mathrm{bp} S V 403 U^{\prime} R$ was replaced by the shorter version (240bp) which is amplified from HMMA006 using primers pair HM\#593/HM\#38 with program $\left(98^{\circ} \mathrm{C} 3^{\prime}\right.$ $\left.98^{\circ} \mathrm{C} 30^{\prime \prime} 59^{\circ} \mathrm{C} 30^{\prime \prime} 72^{\circ} \mathrm{C} 30^{\prime \prime}\right) 5 \mathrm{X}$ followed by $35 \mathrm{X}\left(98^{\circ} \mathrm{C} 30^{\prime \prime} 72^{\circ} \mathrm{C} 1\right)$ ' with final elongation of $7^{\prime}$ at $72^{\circ} \mathrm{C}$. The resulting fragment was digested by BamH//HindII and cloned into HMMA175 giving rise to HMMA251. Finally, the Asc fragment Dm- $\beta 2 t$ : $t$ TA:SV40 from HMMA251 was cloned into the piggyBac transformation vector HMMA331 (chapter 3.4).

To generate spermatogenesis driver construct HMMA335, first 300bp enhancer/promoter region of $D m-d j$ without the $60 \mathrm{bp}$ translation repression element was PCR amplified from gDNA of $D$. melanogaster wild type strain OreR using primers pair HM\#582/HM\#583 and program $98^{\circ} \mathrm{C} 3^{\prime} 54^{\circ} \mathrm{C} 30^{\prime \prime} 72^{\circ} \mathrm{C} 30^{\prime \prime} 5 \mathrm{X}$ followed by $35 \mathrm{X}$ of $98^{\circ} \mathrm{C} 30^{\prime \prime} 63^{\circ} \mathrm{C} 30^{\prime \prime} 72^{\circ} \mathrm{C} 30^{\prime \prime}$ and final elongation of $7^{\prime}$ at $72^{\circ} \mathrm{C}$. digested by $E c o R \mathrm{I} / \mathrm{XbaI}$ and cloned into mfs\#1215 $E c o R I / X b a I$ sites giving rise to HMMA248. The 450 bp $S V 403 U T^{\prime} R$ was replaced by the shorter version (240bp) which is amplified from HMMA006 using primers pair

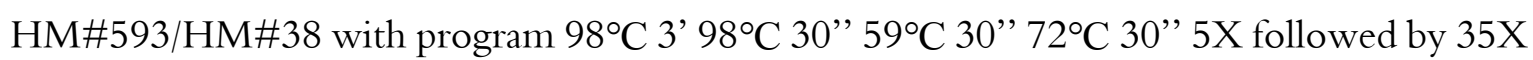
$98^{\circ} \mathrm{C} 30^{\prime \prime} 72^{\circ} \mathrm{C} 1^{\prime}$ with final elongation of $7^{\prime}$ at $72^{\circ} \mathrm{C}$. The resulting fragment was digested by $B a m H I / H i n d I I$ and cloned into HMMA248 giving rise to HMMA252. Finally, the AscI fragment Dm-dj:tTA:SV4Ofrom HMMA252 was then cloned into the piggyBac transformation vector HMMA331 (chapter 3.4).

To generate the Cas 9 responder construct HMMA203, the Asc fragment from HMMA193 containing Cas 9 fused to TREp and the attP site was cloned into the transformation vector mfs 1201 (Scolari et al 2008). HMMA193 was generated by cloning of Ds-b2t 5'UTR amplified from gDNA of $D$. suzukii Italian strain using primer pair HM\#36/HM\#401and cloned into ClaI site of HMMA076 which was generated by cloning of the attP-TREp fragment amplified from mfs1262 into EcoRI/ClaI site of HMMA056 replacing Ds-hsp70 promoter (26).

To generate Cas9 responder construct HMMA356 the Dm Djtranslation suppression element was PCR amplified from gDNA of OreR strain of $D$. melanogaster using primer pair HM\#691/HM\#692 and cloned into BbsI site of HMMA327 (described in chapter 3.5) HMMA366 was generated by cloning of two pairs of annealed oligos HM\#694/HM\#695 and HM\#692/HM\#693 to generate the basal promoter and translation repression element of Dm dj gene respectively into the BbsI/KpnI sites of HMMA327.

To generate plasmid HMMA446 to express $g R N A s$ against D. melanogaster transposable element roo, the annealed oligos HM\#830/HM\#831, HM\#832/HM\#833 and HM\#834/HM\#835 were cloned into plasmid HMMA349 into BbsI, BsaI and SapI sites respectively. 
To generate HMMA447 to express gRNAs against D. melanogaster TE jockey, the annealed oligos HM\#836/HM\#837, HM\#838/HM\#839 and HM\#840/HM\#841 were cloned into plasmid HMMA349 into BbsI, BsaI and SapI sites respectively.

To generate HMMA448 to express $g R N A s$ against D. melanogaster TE 1360 , the annealed oligos HM\#842/HM\#843, HM\#844/HM\#845 and HM\#846/HM\#847 were cloned into plasmid HMMA349 into BbsI, BsaI and SapI sites respectively.

To generate HMMA449 to express gRNAs against D. melanogaster TEs jockey, 1360 and roo at the same time, the annealed oligos HM\#836/HM\#837, HM\#844/HM\#845 and HM\#834/HM\#835 were cloned into plasmid HMMA349 into BbsI, BsaI and SapI sites respectively

The total number of genomic targets for construct HMMA448 with three $g R N A s$ against 1360 are 874, for construct HMMA447 targeting jockey are 212, and for HMMA446 targeting roo are 379. The combination of 1360_gRNA2, roo_gRNA26, and jockey_gRNA1 together in construct HMMA449 should target and induce 659 DSBs

All final construct generated during this study are shown in Table S3.

\section{Germline transformation}

To generate transgenic driver and Cas9 responder lines, piggyBac germline transformation was used. Microinjection of transformation and helper vector at a final concentration of 500 and $300 \mathrm{ng} / \mu \mathrm{L}$ respectively.

All injections for piggyBac germline transformation were performed using the hyperactive helper plasmid MK006 along with the respective transformation vector at a final concentration of a $500 \mathrm{ng} / \mu \mathrm{L}$ and $300 \mathrm{ng} / \mu \mathrm{L}$ respectively. Each $\mathrm{G}_{0}$ fly was crossed individually to three wildtype flies of the opposite sex.

The $F_{1}$ transgenic flies were identified by presence of the respective fluorescent body marker. Screening was performed using Leica M205 FA fluorescence stereomicroscope equipped with camera Q imaging Micropublisher 5.0 RTV (Leica Mikrosysteme Vertrieb Gmb, Wetzlar, 35578 Germany). Transgenic flies were screened using filter sets RFP (excitation: ET546/10x, emission: ET605/70m) or GFP-LP (excitation: ET480/40, emission: ET510 LP). $F_{1}$ transgenic flies were outcrossed to WT flies of the opposite sex.

\section{RT-PCR}

To investigate whether Cas9 expression can be steered by the use of the tet-off binary system, each of the three randomly selected Cas9 responder lines was crossed to different driver lines. Transcription of Cas9 in the testes was detected by RT-PCR. Total RNA, was isolated from testes of 15 individual 4-5 days old adult carrying the driver and responder construct in a heterozygous situation. Dissection was performed in ice cold PBS 1X and testes were transferred to bashing tube with beads and fixed on a vortex and homogenized for 10 minutes. 
RNA was isolated using ZR Tissue \& Insect RNA MicroPrep (Zymo research) according to the manufacturer instructions. Genomic DNA carryover was removed by in-column digestion using turbo-DNase I for 30 minutes at $30^{\circ} \mathrm{C}$.

Second DNase treatment was performed using dsDNase provided with the kit cDNA synthesis kit, first strand cDNA synthesis was done using Maxima First Strand cDNA Synthesis Kit for RT-qPCR, with dsDNase (thermofisher). $1 \mu \mathrm{g}$ RNA was used to make first strand cDNA and $2 \mu \mathrm{L}$ of the cDNA was used for each PCR reaction $n$ a total volume of $25 \mu \mathrm{L}$. to check the expression of cas9, primer pair HM\#619/HM\#135 were used to amplify 460 bp of Cas9. As a negative control the respective Cas9 responder not crossed to driver were used. As a positive control, a pair of primers (HM\#706/HM\#707) that amplify the 3'UTR of $\beta 2 t$ was used which give $250 \mathrm{bp}$. The PCR was performed using $2 \mu \mathrm{L}$ cDNA, $1.25 \mu \mathrm{L}$ forward and 1.25 $\mu \mathrm{l}$ reverse primer $(10 \mu \mathrm{M}), 2 \mu \mathrm{L}$ dNTPs mix and Phusion polymerase in a total reaction of $25 \mu \mathrm{L}$

$15 \mu \mathrm{L}$ of the PCR product was run in $1.5 \%$ agarose gel and documented using UV lamp and a camera attached to a printer. 
Table S1. Primers sequences

\begin{tabular}{|c|c|c|}
\hline Code & Name & Sequence $5^{\prime}-3^{\prime}$ \\
\hline HM\#36 & B2t_XbaI_R3 & CGATTCTAGACATCTTAACCGACTGTCAAGGATC \\
\hline HM\#38 & Hma-45R & TAAGAAGCTTGATACATTGATGAGTTTGGACAAACCAC \\
\hline HM\#135 & cas9-R & GTAGATGGTGGGGTACTTCTCGTGG \\
\hline HM\#351 & Dm_b2t_F & TACCCATGGATTGTAGGAGCCAGAGCCAATGGATC \\
\hline HM\#352 & XbaI_Dm_B2tR & TAATCTAGACATTTTGATAGTAAAGTTAGGGCC \\
\hline HM\#401 & ClaI_Dsb2t_F & CCTCATCGATAGTCCACCCTAGTATCAGCTAGCAAGC \\
\hline HM\#582 & HM_EcoRI_DmDj_F & GCCGAATTCCCTTTAAATATTCTAGTAAAATTCTTTAAG \\
\hline HM\#583 & HM_XbaI_DmDj_R & TAATCTAGACATAAGAATTTTGAAAAAACCACAGC \\
\hline HM\#593 & HM_BamHI_SV40_F & CTAGGATCCGCGGCCGCGACAGATCATAATCAGCCATAC \\
\hline HM\#619 & HM_Cas9_ATG_F & GACGATGACGATAAGGCCCCAAAGAAGAAGCGGAAGGTC \\
\hline HM\#691 & HM_DJTSE_F & $\begin{array}{l}\text { CCATGCTGTGGTTTTTTCAAAATTCTTTGTAAAACTTTTGGTACAA } \\
\text { AATTTAAAAATTTTTCTC }\end{array}$ \\
\hline HM\#692 & HM_DJTSE_R & $\begin{array}{l}\text { ATATGAGAAAAATTTTTAAATTTTGTACCAAAAGTTTTACAAAGAAT } \\
\text { TTTGAAAAAACCACAGC }\end{array}$ \\
\hline HМ\#693 & HM_DJTSE_F2 & $\begin{array}{l}\text { GATCGCTGTGGTTTTTTCAAAATTCTTTGTAAAACTTTTGGTACAA } \\
\text { AАTTTAAAAATTTTTCTC }\end{array}$ \\
\hline HM\#694 & HM_DJ_Pro_F & CTAAACTTGTATAGTTTTGGGGGCAGGTTA \\
\hline HM\#695 & HM_DJ_Pro_R & GATCTAACCTGCCСССAAAACTATACAAGTTTAGGTAC \\
\hline HM\#706 & HM_b2t_3UTR_F & CGAGGATCCTAGGATTAACTTCCCACTCAAGATCACACATG \\
\hline HM\#707 & HM_b2t_3UTR_R & GCCAAGCTTGTCTGCTTATAAATCAACATTTATTCGTAACCC \\
\hline
\end{tabular}


Table S2. Guide RNAs sequences

\begin{tabular}{|c|c|c|}
\hline Code & Name & Sequence 5'-3' \\
\hline HM\#830 & HM_roo_g2_F & TCCAAGGATGGTTGGCACCAGTCA \\
\hline HM\#831 & HM_roo_g2_R & AAACTGACTGGTGCCAACCATCCT \\
\hline HM\#832 & HM_roo_g12_F & AACATTAACCACTGTGGAGGACAC \\
\hline HM\#833 & HM_roo_g12_R & AAACGTGTCCTCCACAGTGGTTAA \\
\hline HM\#834 & HM_roo_g26_F & GCATGCAATATCTACCAGAACCC \\
\hline HM\#835 & HM_roo_g26_R & AACGGGTTCTGGTAGATATTGCA \\
\hline HM\#836 & HM_jockey_g1_F & TCCAGGTTAGGGAGGTCATGAGGG \\
\hline HM\#837 & HM_jockey_g1_R & AAACСССТСАТGACСТСССТАACC \\
\hline HM\#838 & HM_jockey_g2_F & AACACCСТCATGACCTCССТАACC \\
\hline HM\#839 & HM_jockey_g2_R & AAACGGTTAGGGAGGTCATGAGGG \\
\hline HM\#840 & HM_jockey_g10_F & GCATCAACGCACTGTTACCCATG \\
\hline HM\#841 & HM_jockey_g10_R & AACCATGGGTAACAGTGCGTTGA \\
\hline HM\#842 & HM_1360_g1_F & TCCAATGTTCTCAGCGTGAGCGAG \\
\hline HM\#843 & HM_1360_g1_R & AAACCTCGCTCACGCTGAGAACAT \\
\hline HM\#844 & HM_1360_g2_F & AACAGTGGCTCTAGAGGTGGCTCC \\
\hline HM\#845 & HM_1360_g2_R & AAACGGAGCCACCTCTAGAGCCAC \\
\hline HM\#846 & HM_1360_g11_F & GCAATATCTTGAGGCACGAAGTG \\
\hline HM\#847 & HM_1360_g11_R & AACCACTTCGTGCCTCAAGATAT \\
\hline
\end{tabular}


Table S3. List of vectors

\begin{tabular}{|c|c|}
\hline Code & components of the construct \\
\hline HMMA334 & pBXLII $\{$ attP220_B2tE/P:tTA:SV4O_PUb:DsRed:SV4O_attP220\} \\
\hline HMMA345 & pBXLII $\{$ attP220_Dj:tTA:SV4O_PUb:DsRed:SV40_attP220\} \\
\hline HMMA446 & $\operatorname{pSL}\left\{a f \_U 6: 3: r o o 2: 12: 26 \_\beta 2 t: t T A: P 2 A: t G F P: 5 U T R \_a f\right\}$ \\
\hline HMMA447 & 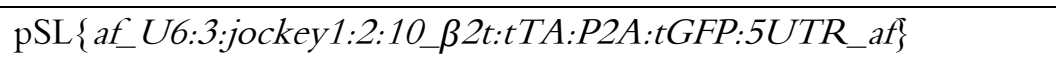 \\
\hline HMMA448 & $\operatorname{pSL}\left\{a f \_U 6: 3: 1360 g 1: 2: 11 \_\beta 2 t: t T A: P 2 A: t G F P: 5 U T R \_a f\right\}$ \\
\hline HMMA449 & pSL $\left\{a f \_U 6: 3: j o c k y 1: 1360 g 2: r o o 26 \_\beta 2 t: t T A: P 2 A: t G F P: 5 U T R \_a f\right\}$ \\
\hline HMMA203 & pBac $\left\{a t t P \_T R E p \beta 2 t 5 U T R: C a s 9 \_P U b: n E G F P: S V 4 O\right\}$ \\
\hline HMMA366 & pBXLII $\{$ attP220_TREp:DjTSE:Cas9_PUb:EGFP:SV40_attP220\} \\
\hline HMMA365 & pBXLII \{attP220_TREDjP:DjTSE:Cas9_PUb:EGFP:SV4O_attP220\} \\
\hline
\end{tabular}




\section{Discussion}

\subsection{Gene drive}

Homing-based gene drive refers to selfish genetic elements that have the ability to target the sister chromosome that lacks them and allow the cell repair mechanism to copy them by homology directed repair in a process called homing (145). Such selfish genetic elements are either natural or synthetically engineered, do not obey the Mendelian law of inheritance, and are therefore passed on to the off-spring more often than can be explained by Mendelian inheritance. Homing endonuclease genes (HEG) are an example of naturally occurring selfish genetic elements that have been proposed for engineering of wild populations of diseases vectors more than 15 years ago (145). The hope was to use them to introduce or delete genes to affect the fertility or to impair the ability to transmit diseases, leading to population suppression or replacement, respectively. Unfortunately, it was not possible to engineer HEG to target sequences other than their natural targets and therefore it was not possible to use them for a custom-made gene drive. The HEG I-Ppolis by chance highly specific to clusters of rDNA that reside in the $\mathrm{X}$ chromosome of the malaria vector Anopheles gambiae $(145,149,150)$. It therefore offered a good model to test the feasibility of using HEG to engineer a meiotic sex bias gene drive by targeting and destroying the $\mathrm{X}$ chromosome during spermatogenesis and thereby bias the sex ratio toward males $(150,151)$. Use of ZFNs and TALENs to engineer gene drive was not successful due to the repetitive nature of the sequence coding for the endonuclease and therefore instability during homing (152). Only after the recent genome editing revolution exploded by the discovery of the potential of using an RNA-guided programable endonuclease to manipulate the genomes of organisms, have scientists had the chance to custom-make and explore the possibilities of gene drive $(138,139,153,154)$. Despite the fact that they may have unpredicted even catastrophic ecological implication, CRISPR/Cas9-based gene drives hold big promises for ecological engineering including conservation of biodiversity, control of invasive species, and of course in pest and vector control (155).

Several synthetic gene drives based on CRISPR/Cas9 system were designed and tested for their functionality. They fall in one of two general groups: suppression gene drive, intended to decrease the population of the target insect and maybe eventually eradicate it completely from the eco-system, or replacement gene drive to render a population unable to transmit a certain disease (156). For the suppression drives, eradication might sound great but it can have unforeseen ecological consequences, especially when targeting endogenous species that are part of the ecological balance (157). In this case, if the gene drive was very efficient to the point of eradication of a species, a vacuum in the system would be created that would have to be filled again. As a consequence, another species that was not a major vector might fill this 
gap and our fight might have to start again from scratch. This is obvious already with insecticides and the emergence of new major pests. However, when targeting invasive species that are still not established in the new ecosystem, this species can be eradicated without serious consequences. And here it should be emphasized that what is meant by eradication is a localized and not global eradication

The second group of gene drives, the replacement drives, was initially proposed to replace populations of diseases' vectors by introducing into the population a gene drive that targets genes necessary for pathogen transmission to reduce or completely abolish their ability to transmit that disease (145). In this case, if the drive does not impose a fitness cost, it should reach fixation in the target population over many generations (145). Replacement gene drives have also been proposed to reverse insecticide resistance by including within the drive cassette the wild type version of the gene that renders the insect susceptible for a give insecticide or groups of insecticides with the same mode of action. For example, point mutations in the voltage-gated sodium channel gene render insects resistant to pyrethroid insecticides due to target site insensitivity (158). This mutation is referred to as knockdown resistance $(k d r)(159)$ and can be reversed by including within the cassette a wild type version of the gene to restore the sensitivity to pyrethroids. Some scientists have even thought about using gene drive to restore susceptibility of weeds to herbicides (160), however, due to cross pollination, the susceptibility could be introgressed into crop plants and as a result the respective group of herbicides could not be used any longer.

To investigate the molecular events and the dynamic of a CRISPR/Cas9-based gene drives we built a sex conversion suppression gene drive targeting $D$. melanogaster female sex determination gene transformer (tra) (161). The main finding was that CRISPR/Cas9-based gene drive creates a hotspot for resistance evolution against the drive itself. Induction of DSB is catastrophic for the cell and has to be immediately repaired. The cell repairs such damage by either of two major pathways, the error prone NHEJ or the more precise HDR (162). When targeting an important gene such as tra, which when knocked out in Drosophila leads to sterile intersexes, the drive exerts high fitness cost and therefore evolution favours selection for resistant alleles. We observed resistance at the very first generation and after 15 generation the resistance reached fixation in the laboratory population of Drosophila. One possible solution to overcome rapid emergence of resistance against gene drive is by using multiple gRNAs. It is very unlikely that several DSBs in the same gene be repaired in a way that keep a functional gene. However, this is not the only way resistance can evolve. Failure to copy the whole drive cassette during homing is another source for emergence of resistance that we have observed. It can also appear as a result of a mutation in the Cas9 or the gRNA cassette that leads to loss of activity. 
Such sex conversion suppression gene drives could be used in the Tephritid fruit flies, where targeting of tra leads to fertile XX males (163). Over the time this should lead to population collapse due to lack of males. Despite all the promises, gene drives with the current designs do not seem to fulfil the requirements to be implemented into operational vector and pest control programs, yet. Several issues need to be addressed such as resistance development, unintended migration of insects carrying drive elements into neighbouring populations, the need to release vast numbers of insects carrying the drive element particularly in the case of thresholddependent gene drive (164). In addition, and despite several new designs that have taken into consideration gene drive containment, reliable methods for reversal of the drive, in case of unexpected outcomes are still needed.

\subsection{The tools of the trade}

Recent advancement in insect genome manipulation tools offers the opportunity for development of transgene-based insect pest control strategies such as gene drive mentioned in 4.1 above as well as improvements to the SIT, which will be discussed in 4.3 below. The use of transposon-based germline transformation has so far been the main tool in insect genetic engineering, whether in basic research or applied biotechnology. Different transposon systems were used to introduce transgenes into the genome of insects including the P-element (68), Tc1/mariner, Minos (165), Hermes (166), and piggyBac (84). Moreover, such vectors were also intensively used for functional genetic screens by insertional mutagenesis, enhancer and gene traps $(74,167)$. The most commonly used vectors so far are based on the lepidopteran transposon piggyBac, which has been shown to function in many different species. The generation of a mutant hyperactive version of the piggyBac transposase with the optimized codons for mammalian cells (85), inspired entomologists to test it in different insects (168). Improvement in piggyBac germline transformation by the use of the hyperactive transposase is a major contribution to researchers in the field of insect biotechnology, especially those working with non-model insects such as agricultural pests or diseases vectors. Using classical transposase, researchers had to spend time and resources with less success to obtain transgenic lines or with low efficiency. We compared the efficiency of three versions of the piggyBac transposases, the same person injected the same construct in the same strains of $D$. melanogaster, the flour beetle Tribolium castaneum, and the Medfly C. capitata and we observed significant increase in transgenesis compared to the wildtype version. This is particularly important in the case of Medfly where improvements in the SIT are desired. Importantly, this increase in efficiency is not accompanied by multiple integrations of the vector. Our results of using the hyperactive transposase disagree with previous work (168), which concluded that this transposase not only does not increase the efficiency of germline transformation, but increases the rate of sterility among injected $G_{0}$ flies. In the case of the invasive fruit pest $D$. suzukii, piggyBac germline transformation showed varying efficiency based on published studies (169) and our own experience. This variation can be attributed to 
differences in the genetic background of the different strains used. We compared three WT strains of $D$. suzukii derived from different geographical areas namely, USA, France, and Italy. The results gave a clear indication that the strain AM derived from the French Alps (170) is more suitable for genetic manipulation using piggyBac vectors compared to the other two strains.

In some cases, especially when different enhancers, or different systems are to be tested and compared, the random transposon-based germline transformation is not suitable. Fortunately, the toolbox for insect genetic manipulation offers as an alternative, the use of site-specific recombinases such as Flp/FR T, Cre/Lox and the $\varphi \mathrm{C} 31$ integrase system (86). Unlike the use of transposon-based vectors, site specific recombinases (SSR) lead as the name suggests to integration of the transgene in a pre-defined genomic site. One successfully SSR that has been used in insect biotechnology is derived from the bacteriophage $\varphi \mathrm{C} 31$ which mediates recombination between two non-identical recognition sequences (98). We have established this system for the invasive fruit pest $D$. suzukii as a way to introduce new transgenes into an established transgenic line that harbours one attP recombination site. This should allow for modification of characterized functional transgenic lines for example by integrating spermmarking transgenes into the embryonic driver lines 06_F5M2 (171) and crossing the combined line to a sexing responder line will allow establishment of a transgenic sexing system and marking system in one strain. Another important application of this system - particularly for transgenic strains to be used in operational SIT - is the stabilization of transgenes. This has already been demonstrated in the Medfly and the vinegar fly by removal of one of the TIRs necessary for transposition of the vector in question $(172,173)$. It is also possible - once a good genomic locus is identified and providing that the transgene contains at least one attPsite - to use the $\varphi \mathrm{C} 31$ system to integrate a new transgene and in a second step to remove the old one along with either of the two ITRs and thus end up with only the new transgene, which is at the same time stabilized. In fact, with the use of the programable genome editing system CRISPR/Cas9 already established for many insect pests including $D$. suzukii (171) and diseases vectors (174), it is possible to introduce by HDR an attPsite in a transgenic line that doesn't have it but has been identified to be at a suitable genomic site. This approach however, leads to the integration of the whole plasmid including the antibiotic resistance gene and therefore always requires a second step for removal of the plasmid backbone.

Another way of using site-specific recombinases is cassette exchange in which reciprocal recombination happens between two sites integrated in the genome by transgenesis and two sites in the donor plasmid flanking the transgene of interest (172). In the case of $\varphi \mathrm{C} 31 \mathrm{RMCE}$, reciprocal recombination takes place between two inverted attP sites in the genome, ideally flanking a marker gene and two inverted $a t t B$ sites in a donor plasmid flanking the transgene of interest plus a different marker in the presence of $\varphi \mathrm{C} 31$ integrase. The advantage of this system over integration in one site is that, only the desired construct is integrated. We 
generated a set of piggyBac vectors suitable for generation of docking lines, and successfully managed to establish the $\varphi \mathrm{C} 31 \mathrm{RMCE}$ (chapter 3.4). We furthermore developed selfdocking lines that expresses $\varphi \mathrm{C} 31$ from the $E / P$ of the maternal effect gene nanos (chapter 3.4). Those lines can be evaluated and categorized according to their suitability to allow expression of transgenes at different stages and in different tissues. That will then reduce the efforts required to test different system by choosing the right set of docking lines according to when and in which tissue the transgene should be expressed. One important advantage of using the $\varphi \mathrm{C} 31$ system is the possibility of integration of large genetic constructs which is not the case with transposon-based transformation vectors.

The recent revolution in genome editing ignited by the discovery that the bacterial adaptive immune system CRISPR/Cas can be used to manipulate genes has led to adoption of the system to many insect pests and diseases vectors in the hope to use it for engineering biotechnological pest control strategies. Previous studies in the invasive pest $D$. suzukii used either plasmids derived from $D$. melanogaster to drive expression of $\operatorname{Cas} 9$ and $g R N A$ or they used Cas9 protein along with in vitro transcribed $g R N A(73,170,175,176)$. To use the CRISPR/Cas9 to develop transgenic strains for pest control application, it is important to identify and use endogenous regulatory elements as they are supposed to drive a more reliable expression compared to exogenous ones. In this respect, our work presents a new set of regulatory elements that can be used in genome editing or development of pest control strategies based on CRISPR/Cas9.

One Possible application of the CRISPR/Cas9 system is the design of synthetic gene drive systems (chapter 3.1). However, it can also be used in SIT context to generate reproductive sterility systems (chapter 3.5-3.7) or sexing systems (chapter 4.3.2).

\subsection{Biotechnological improvements of the SIT}

The SIT as defined by the International Plant Protection Convention 'is a method of pest control using area-wide inundative releases of sterile insects to reduce fertility of a field population of the same species (39). It involves mass rearing of the target pest, removal of females by any of different strategies, sterilization using gamma radiation and inundative successive release to suppress wild populations of the pest. It is obvious that SIT is intended for the control of sexually reproducing insects, however, there are other questions to be answered before taking a decision of using the SIT against a particular pest or disease vector. The question of sexing or precisely the removal of females is of paramount importance. This applies particularly to female mosquitoes which even if sterile can still bite and transmit diseases, and fruit flies such as $C$. capitata that use their ovipositor to sting the fruits. This constrain can be alleviated by the development of a method for sex separation to allow removal of the females before release of the males, which we will discussed in section 4.3.1 below. 
Does the insect in question tolerate high doses of radiation to render them sterile without strong effect in their competence? If radiation affects the ability of sterile males to search, find, and compete with wild type males in mating with wild type females and or the ability of the sperm to compete with the wild type sperm in fertilizing the egg, then it is imperative to find different strategy to induce reproductive sterility. Here, biotechnological approaches offer alternatives to radiation to induce sterility that ensure production of competent males, which I will discuss in section 4.3.2 below. Another aspect of the SIT that can be biotechnologically improved is the marking system, which facilitates monitoring of the released males (section 4.3.3). This has been traditionally achieved by the use of some kind of fluorescent dust.

\subsubsection{Sexing systems}

One of the most critical steps in the establishment of SIT for a particular insect pest or disease vector is to develop a method for sex separation. Ideally the system should act as early as possible to allow removal of females during embryonic development and thereby reduce the cost incurred and space required for raising double the number of insects, when only the males are the actual sex to be released. Historically different approaches were exploited to sex insects including temperature sensitive lethal phenotypes induced by chemical and radiation mutagenesis, as well as physiological and morphological characteristics. These approaches, however, lack universality and are either species specific (pupal size difference and time of eclosion) or requires to be generated de novo for each species ( $t s h$, which demands decades of efforts and resources.

In this regard genetic engineering offers the tools necessary for the development of more generic approaches for sexing. For example, a transgene-based female specific embryonic lethality system (FSEL) has been developed (50-52) based on the knowledge about insect sex determination which mainly relies on sex-specific splicing. The requirements of this system are enhancer/promoters $(E / P)$ of a gene active during early embryonic development, the tetoff binary expression system, a pro-apoptotic gene, and a female specifically spliced intron. Those components together allow the conditional activation of transcription of the proapoptotic effector gene at embryonic stages and the correct splicing only in female embryos and as a consequence female lethality before hatching. Being generic, this system has thus far been transferred to several agricultural pests. Such transgenic sexing system namely strain \#32 developed for the Medfly $C$. capitata (51) has shown comparable performance to the famous genetic sexing strain Vienna8 (49). However, the transgenic strain should be more competent since it has aside from the transgene insertion a clean wild type background compared to the genetic sexing strain generated by random chemical or radiation mutagenesis and translocations causing partial aneuploidy in some progeny

We have prepared the first components to transfer such a FSEL system to the invasive fruit pest $D$. suzukii. We developed two transgenic embryonic driver lines using the $E / P$ of an early 
embryonic gene to drive the expression of the heterologous transcription factor $t T A$. Those lines can be crossed to a hid sexing responder line to generate a female-specific embryonic lethality system for removal of females.

As mentioned earlier, the genetic sexing strain Vienna 8 is the best strain that is currently used in operational area wide SIT. If the molecular basis underlying the tsl and white pupa phenotypes are resolved and providing orthologues of those genes are found in other pests that are suitable for SIT, it should be possible using the CRISPR/Cas9 system to engineer such mutations and translocate rescue alleles to the male-specifying chromosome. However, based on published ts alleles in D. melanogaster (177), it should be possible to engineer these mutations and test them at suitable permissive and non-permissive temperatures. This approach has already been used to engineer a temperature-sensitive allele in the $D$. suzukii transformer-2 gene, which at a non-permissive temperature of $29^{\circ} \mathrm{C}$ leads to conversion of females to infertile intersexes (73). This mutation, if engineered in Tephritid flies, should lead to generation of an elegant sexing system in which the XX female are converted to fertile XX males at the non-permissive temperature, and as a consequence, reduction of the production cost since all the produced biomass is eventually used for release. However, special emphasis should be put on the temperature at which the sex-conversion takes place.

In fact, tsl alleles can be engineered de novo based on the amino acid sequence (178). One approach to achieve that is by targeting predicted buried amino acids in the protein of interest and confirm the burial by replacement of that aa residue with an Asp residue, which is known to inactivate the protein. Then three to four buried aa residues should be replaced for Lys, Ser, Ala, and Trp (178). Once a functional $t s l$ is generated, a wild type rescue allele of the gene should be transferred to the $\mathrm{Y}$ chromosome or its equivalent using CRISPR/Cas9.

\subsubsection{Reproductive sterility}

The beating heart of the SIT is sterilization. If no suitable method to induce reproductive sterility for the target insect is identified, SIT cannot be used. Historically it has been achieved using ionizing radiation $(38,56)$. This has advantages and disadvantages. Among the advantages are the redundancy of the cause of sterility, which is random chromosomal breaks that leaves almost no chance for the development of resistance against SIT. On the other hand, ionizing radiation hits not only the sperm cells but also the somatic cells and therefore adversely affects the fitness of the sterile males to be released (179). Another disadvantage is that not all insects can be made $100 \%$ sterile by radiation, especially lepidopteran pest harbouring holocentric chromosomes (180). Besides, working with radiation can lead to serious health problems. To overcome these disadvantages, scientists exploited molecular biology tools to engineer reproductive sterility systems such as the RIDL based on a tTA positive feed-back loop (61) and transgene-based conditional embryonic lethality systems $(57,58)$, which neither affects the competence of the sterile males nor does it pose health problems to the workers. If a 
combination of FSEL (50-52) with a transgenic reproductive sterility system is to be generated, then a new system for reproductive sterility has to be developed since the conditional embryonic lethality and the FSEL cannot be combined, when based on the same lethality principle. In this regard we proposed the development of a new reproductive sterility system by destroying the function of a specific paternal effect gene (snky) (chapter 3.5). This gene when knocked-out or knocked-down should lead to embryonic lethality due to failure of sperm plasma membrane breakdown and as a consequence failure of preparation of functional male pronucleus $(181,182)$. This system is superior compared to RIDL in that the embryonic development does not continue and larvae are not produced, which represent the most destructive stage in agricultural pests. In the so far published RIDL, the lethality takes place only at the larval or even pupal stages. We proposed two different mechanisms, CRISPR/Cas9 knockout with designed gRNAs to target the gene at a time during spermatogenesis, or RNA interference using a construct that expresses two shRNAs against the gene. These two mechanisms, if combined together in one transgene, should provide a tight reproductive sterility system that should not allow any escapers. Eventually, this reproductive sterility system should be combined with a sexing system (FSEL) (50-52) as well as a marking system (sperm-marking) (62-65) and thereby provide an all in one SIT system for control of the invasive fruit pest $D$. suzukii. This system should be transferable to other insects especially that the gene snky is conserved even in mammals. However, with the genome of many of the major agricultural pest and disease vectors sequenced, it is possible to search for the orthologs in the genome of the target insect. Furthermore, knowledge about the reproductive biology and fertilization is necessary to develop such a system.

Another approach to the generation of a reproductive sterility system is by using CRISPR/Cas9 to induce multiple double strand breaks (DSBs) in the chromosomes of the target insect during spermatogenesis by targeting repetitive sequences such as transposable elements among others as described in (chapter 3.6 and 3.7). The requirements to develop such a system are, $E / P$ of spermatogenesis-specific gene to drive the expression of $t T A$, Cas 9 under the control of $T R E$ and a $g R N A$ cassette driving the expression of multiple $g R N A s$. To realize such a system in $D$. melanogaster, we have chosen euchromatin TEs namely (roo, jockey and 1360 families) as our targets to affect chromosomal breakage. To allow normal sperm production before chromosome shredding by Cas9 we exploited the translational repression element of the $D$. melanogaster dj gene $(183,184)$ to delay the translation of Cas9 mRNA. As for many other genes involved in late spermatogenesis or spermiogenesis, the transcription takes place before the maturation of the spermatocyte at which transcription cessation happens and their mRNA remains arrested (by the action of a translation arrest element mainly residing at the 5'UTR of the genes) until post meiosis.

This approach for inducing reproductive sterility should overcome the deleterious effect of radiation on male fitness. However, the end result should be the same, embryonic aneuploidy 
leading to abortion of embryonic development and thus lethality before hatching. Another important point to mention is that ionizing radiation makes the sterile males disposable, once they are empty of sperm produced before pupal exposure to radiation they are useless, since radiation destroys all spermatogonia. Our proposed system, however, ensures continuous production of sperm since the stage of action is post meiosis and therefore spermatogonia continue to form and differentiate. Therefore, as long as the released males are alive, they should potentially be able to produce sperm (with chromosomal breaks). This system should be easily developed for other insects of agricultural and health relevance since repetitive elements including transposable elements form the bulk of eukaryotic genomes. With next generation sequencing becoming cheaper and the genomes of many insect pests and diseases vectors have already been sequenced, it takes some bioinformatics analysis of the sequences to identify suitable abundant targets. This has already been demonstrated in the major malaria vector Anopheles gambiae where bioinformatics tools were used to identify abundant targets on the $\mathrm{X}$ chromosome and used CRISPR/Cas9 during spermatogenesis to develop $\mathrm{X}$ shredder leading to sex ratio distortion $(141,185)$. The redkmer pipeline for identification of abundant and $\mathrm{X}$-specific CRISPR targets can in principle be used to identify abundant targets that are distributed genome-wide (185). Therefore, the proposed chromosome shredding system offers an alternative to ionizing radiation and should have broad applicability in the establishment of the SIT for the control of many insects.

\subsubsection{Marking}

The sterile males (precisely the pupae) to be release are first dusted with some sort of fluorescent powder to facilitate monitoring and evaluating the success of the control program. $(186,187)$ This is, however, not ideal, since the dye is on the surface and might be washed off by rain, or transferred to wild type males when insects come into contact and also presents health hazards for the facility workers. Again, biotechnology came with an elegant solution that has many applications. The sperm-marking present another biotechnological improvement in the SIT. The idea is to use the $E / P$ of a spermatogenesis-specific gene to drive the expression of a fluorescent protein such as EGFP and DsRed (62-65). Such systems have already been established for some insects by using the $E / P$ of the spermatogenesis specific gene $\beta 2 t$ (62-65). This allows monitoring of the competence of sterile males and their success for mating with wild type females simply by capturing random females from the wild, open the sperm storage organ (spermatheca), and examine it for the presence of marked sperm. This is more accurate and informative than the original dusting approach in practice. It also helps to understand the reproductive biology of the insect to be able to develop more specific pest control strategies. For this purpose, we isolated the spermatogenesis specific gene $\beta 2 t$ of the fruit pest $D$. suzkii and used its $E / P$ to generate a sperm-marking strain that can be used in the SIT against the fly. This strain can also help us to understand more about the reproductive biology, mating behaviour, and post mating response. The system can also be combined with 
other biotechnological improvements such as the sexing system to build strains more suitable for operational SIT. The gene $\beta 2 t$ is spermatogenesis-specific and highly conserved and therefore its $E / P$ presents a good candidate for a generic sperm-marking system based on the isolation of the endogenous $E / P$ in the respective species. 


\section{References}

1. United Nations, Department of Economic and Social Affairs, Population Division. World population prospects Highlights, 2019 revision Highlights, 2019 revision. 2019.

2. Deutsch CA, Tewksbury JJ, Tigchelaar M, Battisti DS, Merrill SC, Huey RB, et al. Increase in crop losses to insect pests in a warming climate. Science. 2018 Aug 31;361(6405):916-9.

3. Global Issues: Food Security [Internet]. [cited 2019 Nov 17]. Available from: https://www.peacecorps.gov/educators/resources/global-issues-food-security/

4. Paini DR, Sheppard AW, Cook DC, De Barro PJ, Worner SP, Thomas MB. Global threat to agriculture from invasive species. Proc Natl Acad Sci USA. 2016 Jul 5;113(27):7575-9.

5. Sharma KR, Jaiswal DK, Babu SR, Saraoj AK. Impact of invasive insect pests species on agroecosystem in India and their management. :8.

6. Mutamiswa R, Chidawanyika F, Nyamukondiwa C. Dominance of spotted stemborer Chilo partellus Swinhoe (Lepidoptera: Crambidae) over indigenous stemborer species in Africa's changing climates: ecological and thermal biology perspectives. Agricultural and Forest Entomology. 2017;19(4):344-56.

7. Kumela T, Mendesil E, Enchalew B, Kassie M, Tefera T. Effect of the Push-Pull Cropping System on Maize Yield, Stem Borer Infestation and Farmers' Perception. Agronomy. 2019 Aug;9(8):452.

8. Yaninek JS, Herren HR. Introduction and spread of the cassava green mite, Mononychellus tanajoa (Bondar) (Acari: Tetranychidae), an exotic pest in Africa and the search for appropriate control methods: a review. Bulletin of Entomological Research. 1988 Mar;78(1):1-13.

9. Yaninek JS. Continental dispersal of the cassava green mite, an exotic pest in Africa, and implications for biological control. Exp Appl Acarol. 1988 Jun 1;4(3):211-24.

10. Shukla PT. Preliminary report on the green mite (Mononychellus tanajoa, Bonder) resistance in Tanzanian local cassava varieties. [Internet]. 1976 [cited 2019 Nov 17]. Available from: https://www.cabi.org/isc/abstract/19780558425

11. Westphal MI, Browne M, MacKinnon K, Noble I. The link between international trade and the global distribution of invasive alien species. Biol Invasions. 2008 Apr;10(4):391-8.

12. Hauser M. A historic account of the invasion of Drosophila suzukii (Matsumura) (Diptera: Drosophilidae) in the continental United States, with remarks on their identification. Pest Management Science. 2011;67(11):1352-7. 
13. Walsh DB, Bolda MP, Goodhue RE, Dreves AJ, Lee J, Bruck DJ, et al. Drosophila suzukii (Diptera: Drosophilidae): Invasive Pest of Ripening Soft Fruit Expanding its Geographic Range and Damage Potential. Journal of Integrated Pest Management. 2011 Apr 1;2(1):G1-7.

14. Mitsui H, Achterberg KV, Nordlander G, Kimura MT. Geographical distributions and host associations of larval parasitoids of frugivorous Drosophilidae in Japan. Journal of Natural History. 2007 Sep 1;41(25-28):1731-8.

15. Bolda MP, Goodhue RE, Zalom FG. Spotted Wing Drosophila: Potential Economic Impact of a Newly Established Pest. :4.

16. Calabria G, Máca J, Bächli G, Serra L, Pascual M. First records of the potential pest species Drosophila suzukii (Diptera: Drosophilidae) in Europe. Journal of Applied Entomology. 2012;136(1-2):139-47.

17. Baroffio C, Fischer S. Neue Bedrohung für Obstplantagen und Beerenpflanzen : Die Kirschessigfliege. [Internet]. [cited 2019 Nov 17]. Available from: https://ira.agroscope.ch/de$\mathrm{CH} /$ publication/28097

18. Vogt H, Baufeld P, Gross J, Koppler K, Hoffmann C. Drosophila suzukii: eine neue Bedrohung für den Europäischen Obst- und Weinbau. Bericht über eine internationale Tagung in Trient, 2. Dezember 2011. Journal für Kulturpflanzen. 2012;64(2):68-72.

19. EPPO Global Database [Internet]. [cited 2019 Nov 17]. Available from: https://gd.eppo.int/reporting/article-2416

20. HARRIS A, SHAW B. First record of Drosophila suzukii (Matsumura) (Diptera, Drosophilidae) in Great Britain. Dipterists Digest. 2014;21:8.

21. Kiss B, Lengyel GD, Kárpáti Z. First record of spotted wing drosophila [Drosophila suzukii (Matsumura, 1931)] in Hungary. [Internet]. 2013 [cited 2019 Nov 17]. Available from: https://www.cabi.org/isc/abstract/20133128433

22. Lengyel GD, Orosz S, Kiss B, Lupták R, Kárpáti Z. New records and present status of the invasive Spotted Wing Drosophila, Drosophila suzukii (Matsumura, 1931) (Diptera) in Hungary. Acta Zool Acad Sci H. 2015;61(1):73-80.

23. Orhan A, Aslantaş R, Önder BŞ, Tozlu G. First record of the invasive vinegar fly Drosophila suzukii (Matsumura) (Diptera: Drosophilidae) from eastern Turkey. Turk J Zool. :4.

24. Deprá M, Poppe JL, Schmitz HJ, De Toni DC, Valente VLS. The first records of the invasive pest Drosophila suzukii in the South American continent. J Pest Sci. 2014 Sep;87(3):379-83. 
25. Lavagnino NJ, Díaz BM, Cichón LI, De la Vega GJ, Garrido SA, Lago JD, et al. New records of the invasive pest Drosophila suzukii (Matsumura) (Diptera: Drosophilidae) in the South American continent. Rev Soc Entomol Arg. 2018 Mar 30;77(1):27-31.

26. Parchami-Araghi M, Gilasian E, Keyhanian A. Spotted Wing Drosophila, Drosophila suzukii (Matsumura) (Dip.: Drosophilidae), an invasive fruit pest new to the Middle East and Iran. Drosophila Information Service. 2015 Jan 1;98:59-60.

27. Burrack HJ, Fernandez GE, Spivey T, Kraus DA. Variation in selection and utilization of host crops in the field and laboratory by Drosophila suzukii Matsumara (Diptera: Drosophilidae), an invasive frugivore. Pest Management Science. 2013;69(10):1173-80.

28. Hauser M, Gaimari S, Damus M. Drosophila suzukii new to North America. Fly Times. $2009 ;(43): 12-5$.

29. Walton V, Lee J, Bruck D, Shearer P, Parent E, Whitney T, et al. Recognizing Fruit Damaged by Spotted Wing Drosophila (SWD), Drosophila suzukii [Internet]. Oregon, USA: Oregon State University Extension Service; 2010 [cited 2019 Nov 17]. Report No.: EM 9021. Available from: https://catalog.extension.oregonstate.edu/sites/catalog/files/project/pdf/em9021.pdf

30. Lee JC, Bruck DJ, Curry H, Edwards D, Haviland DR, Van Steenwyk RA, et al. The susceptibility of small fruits and cherries to the spotted-wing drosophila, Drosophila suzukii. Pest Manag Sci. 2011 Nov;67(11):1358-67.

31. Lee JC, Dreves AJ, Cave AM, Kawai S, Isaacs R, Miller JC, et al. Infestation of Wild and Ornamental Noncrop Fruits by Drosophila suzukii (Diptera: Drosophilidae). Annals of the Entomological Society of America. 2015 Mar 1;108(2):117-29.

32. De Ros G, Conci S, Pantezzi T, Savini G. The economic impact of invasive pest Drosophila suzukii on berry production in\&nbsp;the\&nbsp;Province of Trento, Italy. Journal of Berry Research. 2015 Jan 1;5(2):89-96.

33. Goodhue RE, Bolda M, Farnsworth D, Williams JC, Zalom FG. Spotted wing drosophila infestation of California strawberries and raspberries: economic analysis of potential revenue losses and control costs. Pest Management Science. 2011;67(11):1396-402.

34. Fava ED, Ioriatti C, Melegaro A. Cost-benefit analysis of controlling the spotted wing drosophila (Drosophila suzukii (Matsumura)) spread and infestation of soft fruits in Trentino, Northern Italy. Pest Management Science. 2017;73(11):2318-27.

35. Bruck DJ, Bolda M, Tanigoshi L, Klick J, Kleiber J, DeFrancesco J, et al. Laboratory and field comparisons of insecticides to reduce infestation of Drosophila suzukii in berry crops. Pest Management Science. 2011;67(11):1375-85. 
36. Van Timmeren S, Isaacs R. Control of spotted wing drosophila, Drosophila suzukii, by specific insecticides and by conventional and organic crop protection programs. Crop Protection. 2013 Dec 1;54:126-33.

37. Cuthbertson AGS, Collins DA, Blackburn LF, Audsley N, Bell HA. Preliminary Screening of Potential Control Products against Drosophila suzukii. Insects. 2014 Jun;5(2):488-98.

38. Knipling EF. Possibilities of Insect Control or Eradication Through the Use of Sexually Sterile Males1. Journal of Economic Entomology. 1955 Aug 1;48(4):459-62.

39. Klassen W. Area-Wide Integrated Pest Management and the Sterile Insect Technique. In: Dyck VA, Hendrichs J, Robinson AS, editors. Sterile Insect Technique [Internet]. Berlin/Heidelberg: Springer-Verlag; 2005 [cited 2019 Nov 17]. p. 39-68. Available from: http://link.springer.com/10.1007/1-4020-4051-2_2

40. Klassen W, Curtis CF. History of the Sterile Insect Technique. In: Dyck VA, Hendrichs J, Robinson AS, editors. Sterile Insect Technique: Principles and Practice in Area-Wide Integrated Pest Management [Internet]. Dordrecht: Springer Netherlands; 2005 [cited 2019 Nov 17]. p. 3-36. Available from: https://doi.org/10.1007/1-4020-4051-2_1

41. Klassen W. AREA-WIDE INTEGRATED PEST MANAGEMENT AND THE STERILE INSECT TECHNIQUE. In 2005.

42. Vargas-Terán M, Hofmann HC, Tweddle NE. Impact of Screwworm Eradication Programmes Using the Sterile Insect Technique. In: Dyck VA, Hendrichs J, Robinson AS, editors. Sterile Insect Technique [Internet]. Berlin/Heidelberg: Springer-Verlag; 2005 [cited 2019 Nov 17]. p. 629-50. Available from: http://link.springer.com/10.1007/1-4020-4051$2 \_24$

43. Lindquist DA, Abusowa M, Hall MJR. The New World screwworm fly in Libya: a review of its introduction and eradication. Medical and Veterinary Entomology. 1992;6(1):2-8.

44. Lance D, Mcinnis DO. BIOLOGICAL BASIS OF THE STERILE INSECT TECHNIQUE. In 2005.

45. McInnis DO, Vargas RI. Field Evaluation of a Medfly Genetic Sexing Strain in Hawaii. In: Aluja M, Liedo P, editors. Fruit Flies. New York, NY: Springer; 1993. p. 95-102.

46. Franz G. Recombination between homologous autosomes in medfly (Ceratitis capitata) males: type-1 recombination and the implications for the stability of genetic sexing strains. Genetica. 2002 Sep;116(1):73-84.

47. Kerremans P, Franz G. Cytogenetic analysis of chromosome 5 from the Mediterranean fruit fly, Ceratitis capitata. Chromosoma. 1994 Apr 1;103(2):142-6. 
48. Franz G. Genetic Sexing Strains in Mediterranean Fruit Fly, an Example for Other Species Amenable to Large-Scale Rearing for the Sterile Insect Technique. In 2005.

49. Meza JS, Haq I ul, Vreysen MJB, Bourtzis K, Kyritsis GA, Cáceres C. Comparison of classical and transgenic genetic sexing strains of Mediterranean fruit fly (Diptera: Tephritidae) for application of the sterile insect technique. PLOS ONE. 2018 Dec 14;13(12):e0208880.

50. Schetelig MF, Handler AM. A transgenic embryonic sexing system for Anastrepha suspensa (Diptera: Tephritidae). Insect Biochemistry and Molecular Biology. 2012 Oct;42(10):790-5.

51. Ogaugwu CE, Schetelig MF, Wimmer EA. Transgenic sexing system for Ceratitis capitata (Diptera: Tephritidae) based on female-specific embryonic lethality. Insect Biochemistry and Molecular Biology. 2013 Jan;43(1):1-8.

52. Yan Y, Scott MJ. A transgenic embryonic sexing system for the Australian sheep blow fly Lucilia cuprina. Sci Rep. 2015 Dec;5(1):16090.

53. Patterson RS, Lofgren CS, Boston MD. The Sterile-Male Technique for Control of Mosquitoes: A Field Cage Study with Anopheles quadrimaculatus. The Florida Entomologist. 1968;51(2):77-82.

54. Curtis CF, Grover KK, Suguna SG, Uppal DK, Dietz K, Agarwal HV, et al. Comparative field cage tests of the population suppressing efficiency of three genetic control systems for Aedes aegypti. Heredity. 1976 Feb;36(1):11-29.

55. Msangi AM, Saleh KM, Kiwia N, Mussa WA, Mramba F, Juma KG, et al. Success in Zanzibar: Eradication of tsetse. 2000;(Proceedings: AreaControl of Fruit Flies and Other Insect Pests.International Conferene on Area-Wide Control of Insect Pests, and the 5th International Symposium on Friut Flies of Economic Importance, 28 May-5 June 1998, Penang, Malysia. Penerbit Universiti Sains Malysia, Pulau Pinang, Malysia.):57-66.

56. Bakri A, Mehta K, Lance DR. Sterilizing Insects with Ionizing Radiation. In: Dyck VA, Hendrichs J, Robinson AS, editors. Sterile Insect Technique: Principles and Practice in AreaWide Integrated Pest Management [Internet]. Dordrecht: Springer Netherlands; 2005 [cited 2019 Nov 17]. p. 233-68. Available from: https://doi.org/10.1007/1-4020-4051-2_9

57. Horn C, Wimmer EA. A transgene-based, embryo-specific lethality system for insect pest management. Nat Biotechnol. 2003 Jan;21(1):64-70.

58. Schetelig MF, Caceres C, Zacharopoulou A, Franz G, Wimmer EA. Conditional embryonic lethality to improve the sterile insect technique in Ceratitis capitata (Diptera: Tephritidae). BMC Biol. 2009 Dec;7(1):4. 
59. Gossen M, Bujard H. Tight control of gene expression in mammalian cells by tetracyclineresponsive promoters. Proceedings of the National Academy of Sciences. 1992 Jun 15;89(12):5547-51.

60. Urlinger S, Baron U, Thellmann M, Hasan MT, Bujard H, Hillen W. Exploring the sequence space for tetracycline-dependent transcriptional activators: Novel mutations yield expanded range and sensitivity. Proceedings of the National Academy of Sciences. $2000 \mathrm{Jul}$ 5;97(14):7963-8.

61. Thomas DD, Donnelly CA, Wood RJ, Alphey LS. Insect Population Control Using a Dominant, Repressible, Lethal Genetic System. Science. 2000 Mar 31;287(5462):2474-6.

62. Catteruccia F, Benton JP, Crisanti A. An Anopheles transgenic sexing strain for vector control. Nat Biotechnol. 2005 Nov;23(11):1414-7.

63. Scolari F, Schetelig MF, Bertin S, Malacrida AR, Gasperi G, Wimmer EA. Fluorescent sperm marking to improve the fight against the pest insect Ceratitis capitata (Wiedemann; Diptera: Tephritidae). New Biotechnology. 2008 Jun;25(1):76-84.

64. Zimowska GJ, Nirmala X, Handler AM. The $\beta 2$-tubulin gene from three tephritid fruit fly species and use of its promoter for sperm marking. Insect Biochemistry and Molecular Biology. 2009 Aug;39(8):508-15.

65. Ahmed HMM, Hildebrand L, Wimmer EA. Improvement and Use of CRISPR/Cas9 to Engineer a Sperm-marking Strain for the Invasive Fruit Pest Drosophila suzukii. 2019 [cited 2019 Nov 2]; Available from: https://www.researchsquare.com/article/804bbff3-0228-44d5$8 \mathrm{e} 09-3 \mathrm{acb} 0 \mathrm{~d} 8 \mathrm{e} 408 \mathrm{e} / \mathrm{v} 1$

66. Schetelig MF, Wimmer EA. Insect Transgenesis and the Sterile Insect Technique. In: Vilcinskas A, editor. Insect Biotechnology [Internet]. Dordrecht: Springer Netherlands; 2011 [cited 2019 Nov 3]. p. 169-94. (Biologically-Inspired Systems). Available from: https://doi.org/10.1007/978-90-481-9641-8_9

67. Berghammer AJ, Klingler M, Wimmer EA. A universal marker for transgenic insects. Nature. 1999 Nov;402(6760):370-1.

68. Spradling AC, Rubin GM. Transposition of cloned P elements into Drosophila germ line chromosomes. Science. 1982 Oct 22;218(4570):341-7.

69. Hacker U, Nystedt S, Barmchi MP, Horn C, Wimmer EA. piggyBac-based insertional mutagenesis in the presence of stably integrated P elements in Drosophila. Proceedings of the National Academy of Sciences. 2003 Jun 24;100(13):7720-5.

70. Klemenz R, Weber U, Gehring WJ. The white gene as a marker in a new P-element vector for gene transfer in Drosophila. Nucl Acids Res. 1987;15(10):3947-59. 
71. Handler AM, Ii RAH. Germline transformation of Drosophila melanogaster with the piggyBac transposon vector. Insect Molecular Biology. 1999;9.

72. Masumoto M, Ohde T, Shiomi K, Yaginuma T, Niimi T. A Baculovirus Immediate-Early Gene, ie1, Promoter Drives Efficient Expression of a Transgene in Both Drosophila melanogaster and Bombyx mori. Singh A, editor. PLoS ONE. 2012 Nov 13;7(11):e49323.

73. Li J, Handler AM. Temperature-dependent sex-reversal by a transformer-2 gene-edited mutation in the spotted wing drosophila, Drosophila suzukii. Sci Rep. 2017 Dec;7(1):12363.

74. Horn C, Wimmer EA. A versatile vector set for animal transgenesis. Development genes and evolution. 2000 Dec;210(12):630-7.

75. Caroti F, Urbansky S, Wosch M, Lemke S. Germ line transformation and in vivo labeling of nuclei in Diptera: report on Megaselia abdita (Phoridae) and Chironomus riparius (Chironomidae). Dev Genes Evol. 2015 Jun;225(3):179-86.

76. Gonzalez-Estevez C, Momose T, Gehring WJ, Salo E. Transgenic planarian lines obtained by electroporation using transposon-derived vectors and an eye-specific GFP marker. Proceedings of the National Academy of Sciences. 2003 Nov 25;100(24):14046-51.

77. Holtzman S, Miller D, Eisman RC, Kuwayama H, Niimi T, Kaufman TC. Transgenictools for members of the genus Drosophila with sequenced genomes. Fly. 2010 Oct;4(4):349-62.

78. McClintock B. The origin and behavior of mutable loci in maize. PNAS. 1950 Jun 1;36(6):344-55.

79. Munoz-Lopez M, Garcia-Perez J. DNA Transposons: Nature and Applications in Genomics. CG. 2010 Apr 1;11(2):115-28.

80. Rio DC. Identification and purification of a Drosophila protein that binds to the terminal 31base-pair inverted repeats of the P transposable element. Proc Natl Acad Sci USA. 1988;5.

81. Trubitsyna M, Michlewski G, Finnegan DJ, Elfick A, Rosser SJ, Richardson JM, et al. Use of mariner transposases for one-step delivery and integration of DNA in prokaryotes and eukaryotes by transfection. Nucleic Acids Research. 2017 Jun 2;45(10):e89-e89.

82. Lidholm DA, Lohe AR, Hartl DL. The transposable element mariner mediates germline transformation in Drosophila melanogaster. Genetics. 1993 Jul;134(3):859-68.

83. Medhora MM, MacPeek AH, Hartl DL. Excision of the Drosophila transposable element mariner: identification and characterization of the Mos factor. EMBO J. 1988 Jul;7(7):2185-9. 
84. Cary LC, Goebel M, Corsaro BG, Wang HG, Rosen E, Fraser MJ. Transposon mutagenesis of baculoviruses: analysis of Trichoplusia ni transposon IFP2 insertions within the FP-locus of nuclear polyhedrosis viruses. Virology. 1989 Sep;172(1):156-69.

85. Yusa K, Zhou L, Li MA, Bradley A, Craig NL. A hyperactive piggyBac transposase for mammalian applications. Proceedings of the National Academy of Sciences. 2011 Jan $25 ; 108(4): 1531-6$.

86. Wimmer EA. Insect transgenesis by site-specific recombination. Nat Methods. 2005 Aug;2(8):580-2.

87. Siegal ML, Hartl DL. Transgene Coplacement and high efficiency site-specific recombination with the Cre/loxP system in Drosophila. Genetics. 1996 Oct;144(2):715-26.

88. Long D-P, Zhao A-C, Chen X-J, Zhang Y, Lu W-J, Guo Q, et al. FLP RecombinaseMediated Site-Specific Recombination in Silkworm, Bombyx mori. Liu Z, editor. PLoS ONE. 2012 Jun 29;7(6):e40150.

89. Sauer B. Cre/Iox: One more step in the taming of the genome. Endocr. 2002 Dec $1 ; 19(3): 221-7$.

90. Zheng B, Sage M, Sheppeard EA, Jurecic V, Bradley A. Engineering Mouse Chromosomes with Cre-loxP: Range, Efficiency, and Somatic Applications. Molecular and Cellular Biology. 2000 Jan 15;20(2):648-55.

91. Oberdoerffer P. Unidirectional Cre-mediated genetic inversion in mice using the mutant loxP pair lox66/lox71. Nucleic Acids Research. 2003 Nov 15;31(22):140e-140.

92. Medberry SL, Dale E, Qin M, Ow DW. Intra-chromosomal rearrangements generated by Cre-lox site-specific recombination. Nucl Acids Res. 1995;23(3):485-90.

93. Horn C, Handler AM. Site-specific genomic targeting in Drosophila. Proceedings of the National Academy of Sciences. 2005 Aug 30;102(35):12483-8.

94. Siegal ML, Hartl DL. Application of Cre/loxP in Drosophila. In: Tuan RS, Lo CW, editors. Developmental Biology Protocols: Volume II [Internet]. Totowa, NJ: Humana Press; 2000 [cited 2019 Nov 17]. p. 487-95. (Methods in Molecular Biology ${ }^{\mathrm{TM}}$ ). Available from: https://doi.org/10.1385/1-59259-065-9:487

95. Baer A, Bode J. Coping with kinetic and thermodynamic barriers: RMCE, an efficient strategy for the targeted integration of transgenes. Current Opinion in Biotechnology. 2001 Oct $1 ; 12(5): 473-80$

96. Rausch H, Lehmann M. Structural analysis of the actinophae $\Phi C 31$ attachment site. Nucleic Acids Res. 1991 Oct 11;19(19):5187-9. 
97. Rutherford K, Yuan P, Perry K, Sharp R, Van Duyne GD. Attachment site recognition and regulation of directionality by the serine integrases. Nucleic Acids Res. 2013 Sep;41(17):834156.

98. Thorpe HM, Wilson SE, Smith MCM. Control of directionality in the site-specific recombination system of the Streptomyces phage $\varphi \mathrm{C} 31$. Molecular Microbiology. 2000;38(2):232-41.

99. Bateman JR, Lee AM, Wu C -ting. Site-Specific Transformation of Drosophila via $\phi C 31$ Integrase-Mediated Cassette Exchange. Genetics. 2006 Jun;173(2):769-77.

100. Driever W, Solnica-Krezel L, Schier AF, Neuhauss SC, Malicki J, Stemple DL, et al. A genetic screen for mutations affecting embryogenesis in zebrafish. Development. 1996 Dec;123:37-46.

101. Justice MJ. Capitalizing on large-scale mouse mutagenesis screens. Nat Rev Genet. 2000 Nov;1(2):109-15.

102. Favor J, Neuhäuser-Klaus A. Saturation mutagenesis for dominant eye morphological defects in the mouse Mus musculus. Mamm Genome. 2000 Jul;11(7):520-5.

103. Nadeau JH, Frankel WN. The roads from phenotypic variation to gene discovery: mutagenesis versus QTLs. Nat Genet. 2000 Aug;25(4):381-4.

104. Lanzov VA. Gene Targeting for Gene Therapy: Prospects. Molecular Genetics and Metabolism. 1999 Oct 1;68(2):276-82.

105. Bibikova M, Carroll D, Segal DJ, Trautman JK, Smith J, Kim Y-G, et al. Stimulation of Homologous Recombination through Targeted Cleavage by Chimeric Nucleases. Molecular and Cellular Biology. 2001 Jan 1;21(1):289-97.

106. Bibikova M, Beumer K, Trautman JK, Carroll D. Enhancing gene targeting with designed zinc finger nucleases. Science. 2003 May 2;300(5620):764.

107. Bibikova M, Golic M, Golic KG, Carroll D. Targeted Chromosomal Cleavage and Mutagenesis in Drosophila Using Zinc-Finger Nucleases. Genetics. 2002 Jul 1;161(3):116975.

108. Liang F, Han M, Romanienko PJ, Jasin M. Homology-directed repair is a major doublestrand break repair pathway in mammalian cells. PNAS. 1998 Apr 28;95(9):5172-7.

109. Choulika A, Perrin A, Dujon B, Nicolas JF. Induction of homologous recombination in mammalian chromosomes by using the I-SceI system of Saccharomyces cerevisiae. Mol Cell Biol. 1995 Apr;15(4):1968-73. 
110. Kim YG, Cha J, Chandrasegaran S. Hybrid restriction enzymes: zinc finger fusions to Fok I cleavage domain. Proceedings of the National Academy of Sciences. 1996 Feb 6;93(3):115660 .

111. Miller JC, Tan S, Qiao G, Barlow KA, Wang J, Xia DF, et al. A TALE nuclease architecture for efficient genome editing. Nat Biotechnol. 2011 Feb;29(2):143-8.

112. Reyon D, Tsai SQ, Khayter C, Foden JA, Sander JD, Joung JK. FLASH assembly of TALENs for high-throughput genome editing. Nat Biotechnol. 2012 May;30(5):460-5.

113. Barrangou R, Fremaux C, Deveau H, Richards M, Boyaval P, Moineau S, et al. CRISPR Provides Acquired Resistance Against Viruses in Prokaryotes. Science. 2007 Mar 23;315(5819):1709-12.

114. Makarova KS, Grishin NV, Shabalina SA, Wolf YI, Koonin EV. A putative RNAinterference-based immune system in prokaryotes: computational analysis of the predicted enzymatic machinery, functional analogies with eukaryotic RNAi, and hypothetical mechanisms of action. Biology Direct. 2006 Mar 16;1(1):7.

115. Hsu PD, Lander ES, Zhang F. Development and Applications of CRISPR-Cas9 for Genome Engineering. Cell. 2014 Jun;157(6):1262-78.

116. Cong L, Ran FA, Cox D, Lin S, Barretto R, Habib N, et al. Multiplex Genome Engineering Using CRISPR/Cas Systems. Science. 2013 Feb 15;339(6121):819-23.

117. Bassett AR, Tibbit C, Ponting CP, Liu J-L. Highly Efficient Targeted Mutagenesis of Drosophila with the CRISPR/Cas9 System. Cell Rep. 2013 Jul 11;4(1):220-8.

118. Anders C, Niewoehner O, Duerst A, Jinek M. Structural basis of PAM-dependent target DNA recognition by the Cas9 endonuclease. Nature. 2014 Sep;513(7519):569-73.

119. Hall B, Cho A, Limaye A, Cho K, Khillan J, Kulkarni AB. Genome Editing in Mice Using CRISPR/Cas9 Technology. Current Protocols in Cell Biology. 2018;81(1):e57.

120. Hwang WY, Fu Y, Reyon D, Maeder ML, Tsai SQ, Sander JD, et al. Efficient genome editing in zebrafish using a CRISPR-Cas system. Nat Biotechnol. 2013 Mar;31(3):227-9.

121. Sternberg SH, Redding S, Jinek M, Greene EC, Doudna JA. DNA interrogation by the CRISPR RNA-guided endonuclease Cas9. Nature. 2014 Mar;507(7490):62-7.

122. Lin S, Ewen-Campen B, Ni X, Housden BE, Perrimon N. In Vivo Transcriptional Activation Using CRISPR/Cas9 in Drosophila. Genetics. 2015 Oct 1;201(2):433-42.

123. La Russa MF, Qi LS. The New State of the Art: Cas9 for Gene Activation and Repression. Mol Cell Biol. 2015 Nov 15;35(22):3800-9. 
124. Mali P, Aach J, Stranges PB, Esvelt KM, Moosburner M, Kosuri S, et al. CAS9 transcriptional activators for target specificity screening and paired nickases for cooperative genome engineering. Nat Biotechnol. 2013 Sep;31(9):833-8.

125. Gilbert LA, Horlbeck MA, Adamson B, Villalta JE, Chen Y, Whitehead EH, et al. GenomeScale CRISPR-Mediated Control of Gene Repression and Activation. Cell. 2014 Oct 23;159(3):647-61.

126. Xie N, Zhou Y, Sun Q, Tang B. Novel Epigenetic Techniques Provided by the CRISPR/Cas9 System. Stem Cells Int [Internet]. 2018 Jul 8 [cited 2019 Nov 17];2018. Available from: https:/www.ncbi.nlm.nih.gov/pmc/articles/PMC6079388/

127. Kang JG, Park JS, Ko J-H, Kim Y-S. Regulation of gene expression by altered promoter methylation using a CRISPR/Cas9-mediated epigenetic editing system. Sci Rep. 2019 Aug 19;9(1):1-12.

128. Pulecio J, Verma N, Mejía-Ramírez E, Huangfu D, Raya A. CRISPR/Cas9-Based Engineering of the Epigenome. Cell Stem Cell. 2017 Oct;21(4):431-47.

129. Satomura A, Nishioka R, Mori H, Sato K, Kuroda K, Ueda M. Precise genome-wide base editing by the CRISPR Nickase system in yeast. Sci Rep. 2017 May 18;7(1):1-10.

130. Gopalappa R, Suresh B, Ramakrishna S, Kim H (Henry). Paired D10A Cas9 nickases are sometimes more efficient than individual nucleases for gene disruption. Nucleic Acids Research. 2018 Jul 6;46(12):e71-e71.

131. Trevino AE, Zhang F. Chapter Eight - Genome Editing Using Cas9 Nickases. In: Doudna JA, Sontheimer EJ, editors. Methods in Enzymology [Internet]. Academic Press; 2014 [cited 2019 Nov 17]. p. 161-74. (The Use of CRISPR/Cas9, ZFNs, and TALENs in Generating SiteSpecific Genome Alterations; vol. 546). Available from: http://www.sciencedirect.com/science/article/pii/B9780128011850000088

132. Kim YB, Komor AC, Levy JM, Packer MS, Zhao KT, Liu DR. Increasing the genometargeting scope and precision of base editing with engineered Cas9-cytidine deaminase fusions. Nat Biotechnol. 2017 Apr;35(4):371-6.

133. Zong Y, Wang Y, Li C, Zhang R, Chen K, Ran Y, et al. Precise base editing in rice, wheat and maize with a Cas9-cytidine deaminase fusion. Nat Biotechnol. 2017 May;35(5):438-40.

134. Kim K, Ryu S-M, Kim S-T, Baek G, Kim D, Lim K, et al. Highly efficient RNA-guided base editing in mouse embryos. Nat Biotechnol. 2017 May;35(5):435-7.

135. Cheng T-L, Li S, Yuan B, Wang X, Zhou W, Qiu Z. Expanding C-T base editing toolkit with diversified cytidine deaminases. Nat Commun. 2019 Dec;10(1):3612. 
136. Tan J, Zhang F, Karcher D, Bock R. Engineering of high-precision base editors for sitespecific single nucleotide replacement. Nat Commun. 2019 Jan 25;10(1):1-10.

137. Zetsche B, Gootenberg JS, Abudayyeh OO, Slaymaker IM, Makarova KS, Essletzbichler P, et al. Cpf1 Is a Single RNA-Guided Endonuclease of a Class 2 CRISPR-Cas System. Cell. 2015 Oct;163(3):759-71.

138. Gantz VM, Jasinskiene N, Tatarenkova O, Fazekas A, Macias VM, Bier E, et al. Highly efficient Cas9-mediated gene drive for population modification of the malaria vector mosquito Anopheles stephensi. Proc Natl Acad Sci USA. 2015 Dec 8;112(49):E6736-43.

139. Hammond A, Galizi R, Kyrou K, Simoni A, Siniscalchi C, Katsanos D, et al. A CRISPRCas9 gene drive system targeting female reproduction in the malaria mosquito vector Anopheles gambiae. Nat Biotechnol. 2016 Jan;34(1):78-83.

140. Gantz VM, Bier E. The mutagenic chain reaction: A method for converting heterozygous to homozygous mutations. Science. 2015 Apr 24;348(6233):442-4.

141. Galizi R, Hammond A, Kyrou K, Taxiarchi C, Bernardini F, O’Loughlin SM, et al. A CRISPR-Cas9 sex-ratio distortion system for genetic control. Sci Rep. 2016 Nov;6(1):31139.

142. Werren JH, Nur U, Wu C-I. Selfish genetic elements. Trends in Ecology \& Evolution. 1988 Nov 1;3(11):297-302.

143. Hurst GDD, Werren JH. The role of selfish genetic elements in eukaryotic evolution. Nat Rev Genet. 2001 Aug;2(8):597-606.

144. Chevalier BS. Homing endonucleases: structural and functional insight into the catalysts of intron/intein mobility. Nucleic Acids Research. 2001 Sep 15;29(18):3757-74.

145. Burt A. Site-specific selfish genes as tools for the control and genetic engineering of natural populations. Proc Biol Sci. 2003 May 7;270(1518):921-8.

146. Chatterjee P, Jakimo N, Jacobson JM. Minimal PAM specificity of a highly similar SpCas9 ortholog. Science Advances. 2018 Oct 1;4(10):eaau0766.

147. Kleinstiver BP, Prew MS, Tsai SQ, Topkar VV, Nguyen NT, Zheng Z, et al. Engineered CRISPR-Cas9 nucleases with altered PAM specificities. Nature. 2015 Jul;523(7561):481-5.

148. Kleinstiver BP, Prew MS, Topkar VV, Tsai SQ, Joung JK. 58. Engineered Cas9 Variants with Novel PAM Specificities Expand the Targeting Range of CRISPR/Cas Nucleases. Molecular Therapy. 2015 May 1;23:S26. 
149. Galizi R, Doyle LA, Menichelli M, Bernardini F, Deredec A, Burt A, et al. A synthetic sex ratio distortion system for the control of the human malaria mosquito. Nat Commun. 2014 Sep;5(1):3977.

150. Windbichler N, Papathanos PA, Catteruccia F, Ranson H, Burt A, Crisanti A. Homing endonuclease mediated gene targeting in Anopheles gambiae cells and embryos. Nucleic Acids Research. 2007 Aug 23;35(17):5922-33.

151. Windbichler N, Papathanos PA, Crisanti A. Targeting the X Chromosome during Spermatogenesis Induces Y Chromosome Transmission Ratio Distortion and Early Dominant Embryo Lethality in Anopheles gambiae. PLOS Genetics. 2008 Dec 5;4(12):e1000291.

152. Simoni A, Siniscalchi C, Chan Y-S, Huen DS, Russell S, Windbichler N, et al. Development of synthetic selfish elements based on modular nucleases in Drosophila melanogaster. Nucleic Acids Res. 2014 Jun 17;42(11):7461-72.

153. Kyrou K, Hammond AM, Galizi R, Kranjc N, Burt A, Beaghton AK, et al. A CRISPR-Cas9 gene drive targeting doublesex causes complete population suppression in caged Anopheles gambiae mosquitoes. Nat Biotechnol. 2018 Nov;36(11):1062-6.

154. Webster SH, Vella MR, Scott MJ. Development and testing of a novel Killer-Rescue selflimiting gene drive system in Drosophila melanogaster [Internet]. Genetics; 2019 Jun [cited 2019 Oct 10]. Available from: http://biorxiv.org/lookup/doi/10.1101/680629

155. Taylor HR, Gemmell NJ. Emerging Technologies to Conserve Biodiversity: Further Opportunities via Genomics. Response to Pimm et al. Trends in Ecology \& Evolution. 2016 Mar;31(3):171-2.

156. Rode NO, Estoup A, Bourguet D, Courtier-Orgogozo V, Débarre F. Population management using gene drive: molecular design, models of spread dynamics and assessment of ecological risks. Conserv Genet. 2019 Aug;20(4):671-90.

157. Esvelt KM, Smidler AL, Catteruccia F, Church GM. Concerning RNA-guided gene drives for the alteration of wild populations. Tautz D, editor. eLife. 2014 Jul 17;3:e03401.

158. Dong K. Insect sodium channels and insecticide resistance. Invert Neurosci. 2007;14.

159. Zhou X, Yang C, Liu N, Li M, Tong Y, Zeng X, et al. Knockdown resistance (kdr) mutations within seventeen field populations of Aedes albopictus from Beijing China: first report of a novel V1016G mutation and evolutionary origins of kdr haplotypes. Parasites Vectors. 2019 Dec;12(1):180.

160. Neve P. Gene drive systems: do they have a place in agricultural weed management?: Gene drive and weed management. Pest Manag Sci. 2018 Dec;74(12):2671-9. 
161. Baker BS. Sex in flies: the splice of life. Nature. 1989 Aug;340(6234):521-4.

162. Featherstone C, Jackson SP. DNA double-strand break repair. 1999;9(20):3.

163. Pane A, Salvemini M, Bovi PD, Polito C, Saccone G. The transformer gene in Ceratitis capitata provides a genetic basis for selecting and remembering the sexual fate. Development. 2002 Aug 1;129(15):3715-25.

164. Leftwich PT, Edgington MP, Harvey-Samuel T, Carabajal Paladino LZ, Norman VC, Alphey L. Recent advances in threshold-dependent gene drives for mosquitoes. Biochemical Society Transactions. 2018 Oct 19;46(5):1203-12.

165. Pavlopoulos A, Oehler S, Kapetanaki MG, Savakis C. The DNA transposon Minos as a tool for transgenesis and functional genomic analysis in vertebrates and invertebrates. Genome Biology. 2007;7.

166. Warren WD, Atkinson PW, O'Brochta DA. The Hermes transposable element from the house fly, Musca domestica, is a short inverted repeat-type element of the hobo, Ac, and Tam3 (hAT) element family. Genet Res. 1994 Oct;64(2):87-97.

167. Horn C, Offen N, Nystedt S, Häcker U, Wimmer EA. piggyBac-Based Insertional Mutagenesis and Enhancer Detection as a Tool for Functional Insect Genomics. Genetics. 2003 Feb 1;163(2):647-61.

168. Wright JA, Smith RC, Li X, Craig NL, Atkinson PW. IPB7 transposase behavior in Drosophila melanogaster and Aedes aegypti. Insect Biochem Mol Biol. 2013 Oct;43(10):899906.

169. Schetelig MF, Handler AM. Germline transformation of the spotted wing drosophilid, Drosophila suzukii, with a piggyBac transposon vector. Genetica. 2013 Jun 1;141(4):189-93.

170. Karageorgi M, Bräcker LB, Lebreton S, Minervino C, Cavey M, Siju KP, et al. Evolution of Multiple Sensory Systems Drives Novel Egg-Laying Behavior in the Fruit Pest Drosophila suzukii. Current Biology. 2017 Mar;27(6):847-53.

171. Ahmed HMM, Hildebrand L, Wimmer EA. Improvement and use of CRISPR/Cas9 to engineer a sperm-marking strain for the invasive fruit pest Drosophila suzukii. BMC Biotechnol. 2019 Dec;19(1):85.

172. Schetelig MF, Scolari F, Handler AM, Kittelmann S, Gasperi G, Wimmer EA. Site-specific recombination for the modification of transgenic strains of the Mediterranean fruit fly Ceratitis capitata. Proceedings of the National Academy of Sciences. 2009 Oct 27;106(43):18171-6. 
173. Handler AM, Zimowska GJ, Horn C. Post-integration stabilization of a transposon vector by terminal sequence deletion in Drosophila melanogaster. Nat Biotechnol. 2004 Sep;22(9):11504.

174. Meredith JM, Underhill A, McArthur CC, Eggleston P. Next-Generation Site-Directed Transgenesis in the Malaria Vector Mosquito Anopheles gambiae: Self-Docking Strains Expressing Germline-Specific phiC31 Integrase. PLOS ONE. 2013 Mar 13;8(3):e59264.

175. Kalajdzic P, Schetelig MF. CRISPR/Cas-mediated gene editing using purified protein in Drosophila suzukii. Entomol Exp Appl. 2017 Sep;164(3):350-62.

176. Li F, Scott MJ. CRISPR/Cas9-mediated mutagenesis of the white and Sex lethal loci in the invasive pest, Drosophila suzukii. Biochemical and Biophysical Research Communications. 2016 Jan;469(4):911-6.

177. Ezell D, Suzuki DT. TEMPERATURE-SENSITIVE MUTATIONS IN DROSOPHILA MELANOGASTER. IX. DOMINANT COLD-SENSITIVE LETHALS ON THE AUTOSOMES. Genetics. 1972;70:12.

178. Chakshusmathi G, Mondal K, Lakshmi GS, Singh G, Roy A, Ch. RB, et al. Design of temperature-sensitive mutants solely from amino acid sequence. Proceedings of the National Academy of Sciences. 2004 May 25;101(21):7925-30.

179. Barry JD, McInnis DO, Gates D, Morse JG. Effects of irradiation on Mediterranean fruit flies (Diptera: Tephritidae): emergence, survivorship, lure attraction, and mating competition. J Econ Entomol. 2003 Jun;96(3):615-22.

180. Marec F, Vreysen MJB. Advances and Challenges of Using the Sterile Insect Technique for the Management of Pest Lepidoptera. Insects. 2019 Oct 25;10(11).

181. Wilson KL, Fitch KR, Bafus BT, Wakimoto BT. Sperm plasma membrane breakdown during Drosophila fertilization requires Sneaky, an acrosomal membrane protein. Development. 2006 Dec 15;133(24):4871-9.

182. Fitch KR, Wakimoto BT. The Paternal Effect Gene ms(3)sneaky Is Required for Sperm Activation and the Initiation of Embryogenesis in Drosophila melanogaster. Developmental Biology. 1998 May 15;197(2):270-82.

183. Blümer N, Schreiter K, Hempel L, Santel A, Hollmann M, Schäfer MA, et al. A new translational repression element and unusual transcriptional control regulate expression of don juan during Drosophila spermatogenesis. Mechanisms of Development. 2002 Jan 1;110(1):97112.

184. Hempel LU, Rathke C, Raja SJ, Renkawitz-Pohl R. In Drosophila, don juan and don juan like encode proteins of the spermatid nucleus and the flagellum and both are regulated at the 
transcriptional level by the TAFII80 cannonball while translational repression is achieved by distinct elements. Developmental Dynamics. 2006;235(4):1053-64.

185. Papathanos PA, Windbichler N. Redkmer: An Assembly-Free Pipeline for the Identification of Abundant and Specific X-Chromosome Target Sequences for X-Shredding by CRISPR Endonucleases. CRISPR J. 2018 Feb 1;1(1):88-98.

186. Lees RS, Gilles JR, Hendrichs J, Vreysen MJ, Bourtzis K. Back to the future: the sterile insect technique against mosquito disease vectors. Current Opinion in Insect Science. 2015 Aug $1 ; 10: 156-62$.

187. Parker AG. Mass-Rearing for Sterile Insect Release. In: Dyck VA, Hendrichs J, Robinson AS, editors. Sterile Insect Technique: Principles and Practice in Area-Wide Integrated Pest Management [Internet]. Dordrecht: Springer Netherlands; 2005 [cited 2019 Nov 21]. p. 20932. Available from: https://doi.org/10.1007/1-4020-4051-2_8 


\section{Curriculum Vitae}

M.Sc. Genetics \& Molecular Biology

Hassan Mutasim Mohammed Ahmed

Department of Developmental Biology

JFB-Institute of Zoology and Anthropology

Georg-August-University Goettingen

GZMB, Ernst-Caspari-Haus

Justus-von-Liebig-Weg 11

37077 Göttingen

Germany

\section{Education}

01/10/2014-

20.12 .2019

2008-2011

2000-2005

1999- 2000
Dr. rer.nat. 'BBiology" Georg-August-Universität Göttingen, Göttingen (Germany). Topic: Development of Transgenic Sterile Insect Technique Strains for the Invasive Fruit pest Drosophila suzukii.

M.Sc. Genetics \& Molecular Biology, Department of Zoology University of Khartoum, Khartoum (Sudan).

Compulsary: Genetics, Molecular Biology, Biochemistry, Physiology, Bacteriology, Virology, Parasitology, Statistics, Techniques and Instrumentations. Elective: Human Immunology, Human Genetics and Human infectious diseases

B.Sc. (Honours) Agric. Entomology "First Class", Faculty of Agriculture - University of Khartoum, Khartoum North (Sudan) Compulsary: five semesters covering all aspects of biological sciences, chemistry, physical chemistry, biochemistry, physics, Mathematics, English, Arabic.

Elective: five semesters covering the field of Entomology, pest control, plant pathology, and pesticide science

Sudan high school certificate, choice "Biology" grade 83.4\%

Additional skills:

Languages

Computer skills
- Arabic (mother tongue)

- English second language (fluent)

- German third language (fairly good)

- Microsoft package

- Basic linux

- Adobe Ilustrator 
Awards \& Grants - Khartoum University prize for the best performace in Crop protection

- DAAD scholarship for PhD studies

\section{Conferences \& Meetings}

- The 1st FAO/IAEA Research Coordination Meeting (RCM) of the Coordinated Research Project (CRP) on "Generic approach for the development of genetic sexing strains for SIT applications" 7-11 October 2019

- Third FAO/IAEA Research Coordination Meeting (RCM) on "Comparing Rearing Efficiency and Competitiveness of Sterile Male Strains Produced by Genetic, Transgenic or Symbiont-based Technologies” Bangkok, Thailand 18-22 June 2018

- Göttingen Biotech Symposium, German Primate Center DPZ, Göttingen, Germany, 26 September 2017 'Horizons in Molecular Biology Symposium. Max Plank Institute for biophysical Chemistry, Göttingen, Germany, 11-14 September 2017

- Third FAO-IAEA International Conference on Area-wide Management of Insect Pests: Integrating the Sterile Insect and Related Nuclear and Other Techniques. Vienna, Austria 22-26 May 2017

\section{Courses}

- RNAseq Data Analysis, organized by The Transcriptome and Genome Analysis Laboratory TAL and the Medical Biometry and Statistical Bioinformatics, Institut für Medizinische Statistik, Universitätsmedizin Göttingen, Göttingen, Germany, 14-15 September 2017

- $\quad$ EMBO Practical Course, Mouse genome engineering, 25 August - 06 September 2019 | Dresden, Germany

\section{Publications}

- Max S. Farnworth, Kolja N. Eckermann, Hassan M.M. Ahmed, Dominik S. Mühlen, Bicheng $\mathrm{He}$, and Gregor Bucher. The Red Flour Beetle as Model for Comparative Neural Development: Genome Editing to Mark Neural Cells in Tribolium Brain Development. Brain Development: Methods and Protocols 2019

- KaramiNejadRanjbar M, Eckermann KN, Ahmed HMM, Sánchez C. HM, Dippel S, Marshall JM, et al. Consequences of resistance evolution in a Cas9-based sex conversionsuppression gene drive for insect pest management. Proc Natl Acad Sci USA. 2018 Jun 12;115(24):6189-94.

- Eckermann KN, Ahmed HMM, KaramiNejadRanjbar M, Dippel S, Ogaugwu CE, Kitzmann $\mathrm{P}$, et al. Hyperactive piggyBac transposase improves transformation efficiency in diverse insect species. Insect Biochem Mol Biol. 2018 Jul 1; 98:16-24

- Eckermann KN, Dippel S, KaramiNejadRanjbar M, Ahmed HM, Curril IM, Wimmer EA. Perspective on the combined use of an independent transgenic sexing and a multifactorial reproductive sterility system to avoid resistance development against transgenic Sterile Insect Technique approaches. BMC Genet. 2014;15(Suppl 2): S17

Signature 